University of Louisville

ThinkIR: The University of Louisville's Institutional Repository

Electronic Theses and Dissertations

$12-2016$

\title{
Simulation modeling for energy consumption of residential consumers in response to demand side management.
}

Prajwal Khadgi

University of Louisville

Follow this and additional works at: https://ir.library.louisville.edu/etd

Part of the Industrial Engineering Commons, and the Operational Research Commons

\section{Recommended Citation}

Khadgi, Prajwal, "Simulation modeling for energy consumption of residential consumers in response to demand side management." (2016). Electronic Theses and Dissertations. Paper 2600.

https://doi.org/10.18297/etd/2600

This Doctoral Dissertation is brought to you for free and open access by ThinkIR: The University of Louisville's Institutional Repository. It has been accepted for inclusion in Electronic Theses and Dissertations by an authorized administrator of ThinkIR: The University of Louisville's Institutional Repository. This title appears here courtesy of the author, who has retained all other copyrights. For more information, please contact thinkir@louisville.edu. 
SIMULATION MODELING FOR ENERGY CONSUMPTION OF RESIDENTIAL CONSUMERS IN RESPONSE TO DEMAND SIDE MANAGEMENT

\author{
By \\ Prajwal Khadgi \\ B.E., Tribhuvan University, 2005 \\ M.S., Northern Illinois University, 2009
}

\begin{abstract}
A Dissertation
Submitted to the Faculty of the

University of Louisville

J.B. Speed School of Engineering

in Partial Fulfillment of the Requirements

for the Degree of

Doctor of Philosophy

in Industrial Engineering
Department of Industrial Engineering
University of Louisville
Louisville, Kentucky

December, 2016 

SIMULATION MODELING FOR ENERGY CONSUMPTION OF RESIDENTIAL CONSUMERS IN RESPONSE TO DEMAND SIDE MANAGEMENT

\author{
By \\ Prajwal Khadgi \\ B.E., Tribhuvan University, 2005 \\ M.S., Northern Illinois University, 2009 \\ Dissertation Approved on
}

November 18, 2016

by the following dissertation committee:

Dr. Lihui Bai

Dr. Gerald W. Evans

Dr. Suraj Alexander

Dr. Michael McIntyre 


\section{DEDICATION}

To my grandfather Mr. Dev Ratna Khadgi. 


\section{ACKNOWLEDGEMENTS}

I would like to first of all extend my deepest appreciation to my advisor, Dr. Lihui Bai, for her continued support, encouragement and guidance throughout this research, as a mentor and a friend. Without her enduring patience and supervision, this milestone would not have been possible. My sincere gratitude also goes to Dr. Gerald Evans for constantly helping me, especially at the beginning of my graduate study as my first advisor. I am also grateful to Glasgow Electric Plant Board for important technical support on behalf of the TVA grant project. Being able to gain access to invaluable data helped me greatly. The knowledge and experience gained while working on the GEPB project is greatly appreciated and valued.

Most importantly, I would like to thank my parents, Roshan Khadgi and Rita Khadgi, for their unconditional love and support. I thank my family for their prayers and their belief in my ability. The constant love and support from my beloved, Agrima Koirala, has been instrumental in my success and I thank her for inspiring me to always achieve greater heights. I wouldn't be in this position without her. Lastly, I thank all my friends who have supported me along the way throughout this unforgettable journey. Thank you all. 


\section{ABSTRACT \\ SIMULATION MODELING FOR ENERGY CONSUMPTION OF RESIDENTIAL CONSUMERS IN RESPONSE TO DEMAND SIDE MANAGEMENT \\ Prajwal Khadgi}

November 18, 2016

Energy efficiency in the electricity distribution system continues to gain importance as demand for electricity keeps rising and resources keep diminishing. Achieving higher energy efficiency by implementing control strategies and demand response (DR) programs has always been a topic of interest in the electric utility industry. The advent of smart grids with enhanced data communication capabilities propels DR to be an essential part of the next generation power distribution system. Fundamentally, DR has the ability to charge a customer the true price of electricity at the time of use, and the general perception is that consumers would shift their load to a cheaper off-peak period. Consequently, when designing incentives most DR literature assumes consumers always minimize total electricity cost when facing energy consumption decisions. However, in practice, it has been shown that customers often override financial incentives if they feel strongly about the inconvenience of load-shifting arrangements.

In this dissertation, an energy consumption model based on consumers ${ }^{\text {ee }}$ response to both cost and convenience/comfort is proposed in studying the effects of differential pricing mechanisms. We use multi-attribute utility functions and a model predictive control mechanism to simulate consumer behavior of using non-thermostatic loads 
(prototypical home appliances) and thermostatically controlled load (HVAC). The distributed behavior patterns caused by risk nature, thermal preferences, household size, etc. are all incorporated using an object-oriented simulation model to represent a typical residential population.

The simulation based optimization platform thus developed is used to study various types of pricing mechanisms including static and dynamic variable pricing. There are many electric utilities that have applied differential pricing structures to influence consumer behavior. However, majority of current DR practices include static variable pricings, since consumer response to dynamic prices is very difficult to predict. We also study a novel pricing method using demand charge on coincident load. Such a pricing

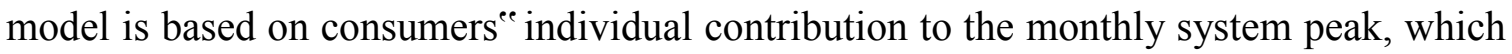
is highly stochastic. We propose to use the conditional Markov chain to calculate the probability that the system will reach a peak, and subsequently simulate consumers"e behavior in response to that peak. Sensitivity analysis and comparisons of various rate structures are done using simulation. Overall, this dissertation provides a simulation model to study electricity consumers ${ }^{\text {ee }}$ response to DR programs and various rate structures, and thus can be used to guide the design of optimal pricing mechanism in demand side management. 


\section{TABLE OF CONTENTS}

PAGE

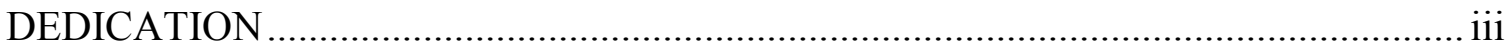

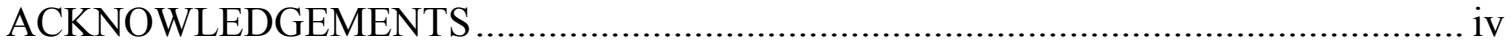

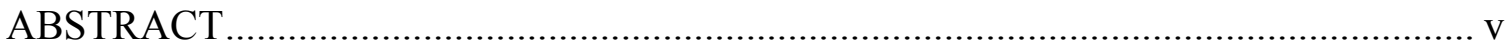

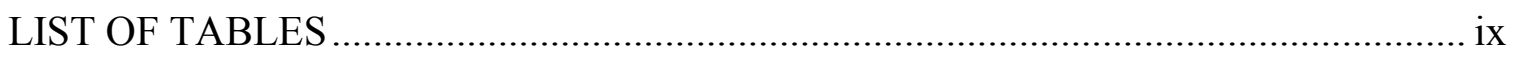

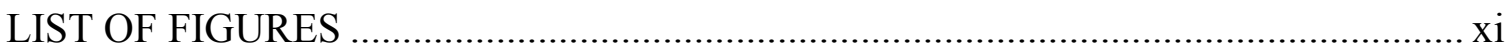

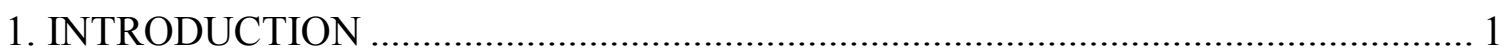

1.1. Demand Response in Residential Sector .................................................... 3

1.2. Centralized and Decentralized Control ................................................................ 7

1.3. Residential Load Control Via Innovative Pricing Models ................................... 10

1.4. Contributions of The Dissertation.................................................................... 12

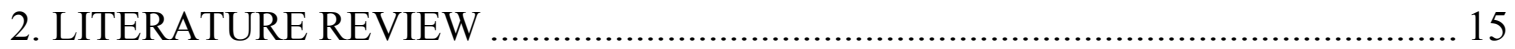

2.1. Demand Response in Smart Grid.................................................................... 15

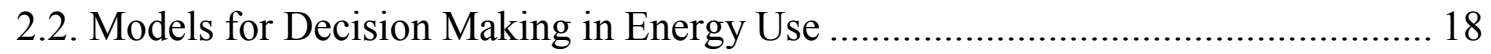

2.3. Thermal Modeling and Model Predictive Control ............................................... 20

2.4. Residential Electricity Rates and DR Practices .............................................. 21

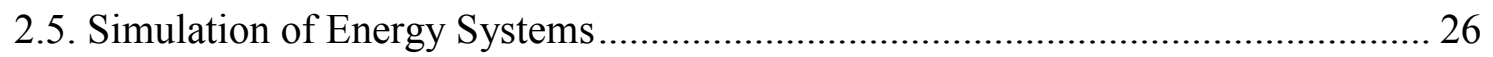

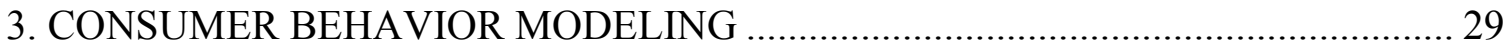

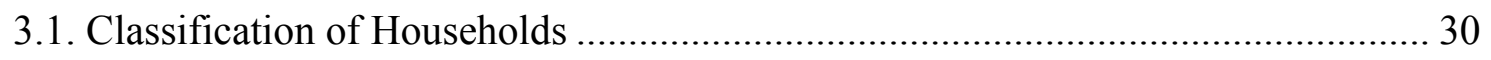

3.2. Multi Attribute Utility (MAU) Functions ................................................... 36

3.2.1. Utility of Money and Exponential Functions............................................. 38

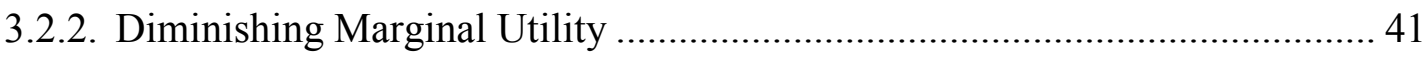

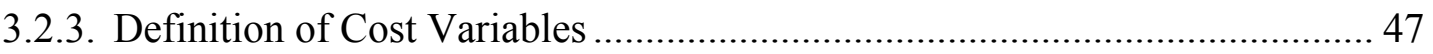

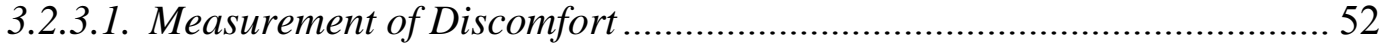

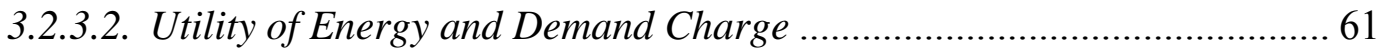




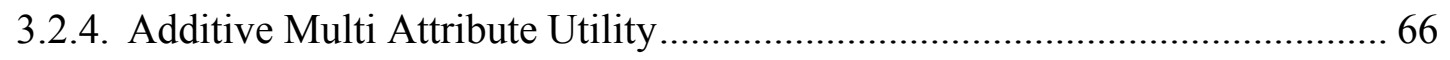

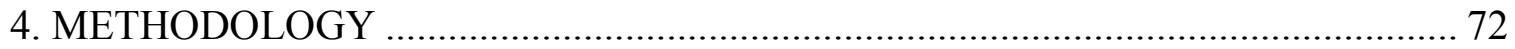

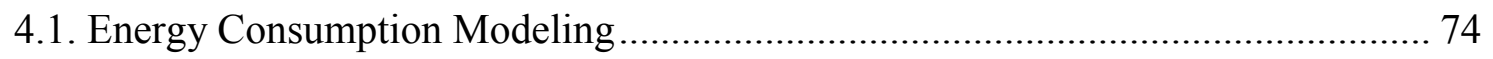

4.1.1. Non-Thermostatic Loads (NTL) Model ……………………………........... 76

4.1.2. Thermostatically Controlled Loads (TCL) Model .......................................... 85

4.2. Model Predictive Control of TCL...................................................................... 94

4.2.1. Thermal Model Parameter Identification........................................................ 99

4.3. Multi Period Optimization .................................................................................... 105

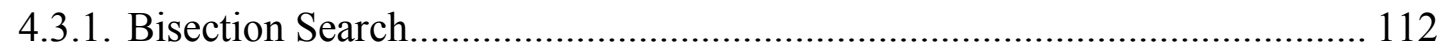

4.4. Peak Forecasting With Conditional Markov Chain ............................................ 114

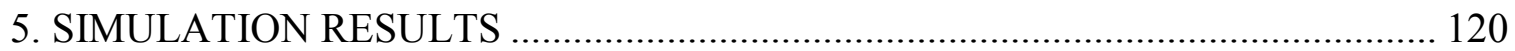

5.1. Model Verification and Load Validation .......................................................... 122

5.2. Design of Experiment for Residential Rates......................................................... 130

5.3. NTL Under Static Pricing ................................................................................. 136

5.4. NTL Under Dynamic Pricing........................................................................... 140

5.4.1. Experimental Design (Latin Hypercube Method) ......................................... 145

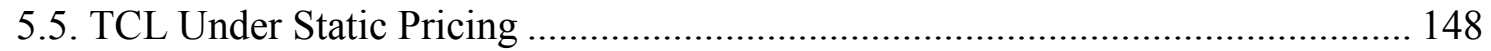

5.6. TCL Under Dynamic Pricing ........................................................................... 158

5.6.1. Discussions on the Effect of Residential Demand Charge ............................. 168

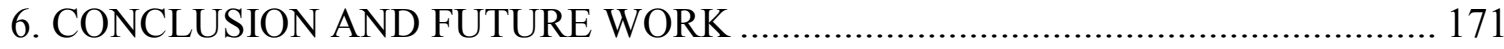

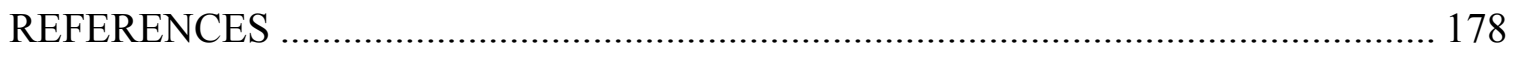

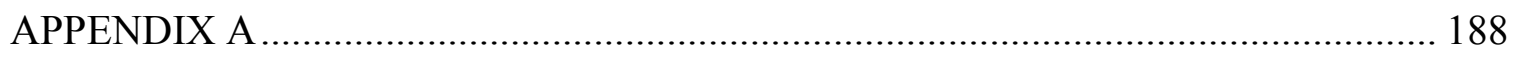

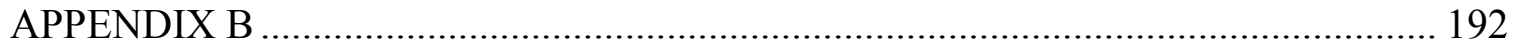

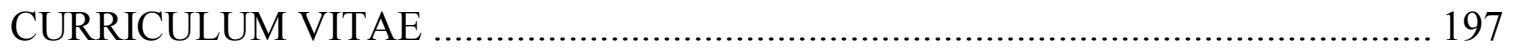




\section{LIST OF TABLES}

Table 1: Classification of households according to annual income................................... 32

Table 2: Classification based on number of occupants................................................... 36

Table 3: Annual and daily demand per person for various appliances .............................. 77

Table 4: Example of fixed and variable convenience values over $24 \mathrm{hr}$.......................... 79

Table 5: Appliance usage durations for various household sizes ...................................... 84

Table 6: Regression analysis for thermal coefficients identification for HOME 01 ...... 103

Table 7: Thermal parameters estimated from fitted regression models for the test homes

Table 8: Table of notations used in the optimization problem ……................................ 107

Table 9: TOU rates used in the NTL model ............................................................ 132

Table 10: Differential pricing models and rate designs ................................................. 135

Table 11: Daily equivalent costs for different pricing models using typical summer load 135

Table 12: Average cost and inconvenience comparison between flat rate and TOU rates 140

Table 13: Group-wise response comparison between flat rate and RTP ....................... 145

Table 14: Sensitivity analysis of RTP for NTL ....................................................... 145

Table 15: Estimated regression coefficients for PAR and VAR for five factor DOE .... 148

Table 16: Estimated regression coefficients for PAR and VAR for ten-factor DOE ..... 148

Table 17: The effect of varying risk nature ............................................................ 153

Table 18: Analysis of TOU vs. flat rate for $\mathrm{n}=30$ households ....................................... 153 
Table 19: Average monthly bill and coincident load factor (LF) for different household types under various static pricing models ....................................................... 156

Table 20: LF and VAR for TCL model under various RTP functions ........................... 162

Table 21: Average monthly bill and coincident load factor (LF) for different household types under various demand charge based dynamic pricing models ................ 166

Table 22: The steady state probabilities of the system being at peak, intermediate and offpeak states under various demand charge based dynamic rates 167 


\section{LIST OF FIGURES}

Figure 1: Total US energy flow, 2014 (EIA, Annual Energy Review, 2015) .................. 2

Figure 2: Distribution of energy in the residential sector (EIA, 2015) ............................. 3

Figure 3: Electricity consumption by end-use sector share for 2013 (EIA, 2015) ............ 3

Figure 4: Distribution of electricity usage in a residential household (EIA, 2015) ........... 4

Figure 5: Residential sector delivered energy intensity for selected end uses (EIA, 2015)

Figure 6: Residential electric rates by service territory in 2013 (NREL, 2016) .............. 24

Figure 7: Histogram of average household annual income for 2010 and 2014 .............. 31

Figure 8: Histogram of annual energy consumption of 1200 homes in Glasgow, KY ..... 32

Figure 9: Load profiles of four different homes showing variable consumption patterns 33

Figure 10: Normal distribution of average load factor for 1200 homes ......................... 35

Figure 11: Annual energy consumption Vs Average Load Factor for 1200 homes ......... 35

Figure 12: General form of the non-linear, increasing utility function of money ............ 40

Figure 13: The value of money gained or lost, as viewed from a reference point........... 43

Figure 14: (a) Unbounded utility function of cost with arbitrarily small utility at upper bound, (b) Bounded utility function of cost with fixed upper bound at zero.. 43

Figure 15: Estimated summer base load for various occupancy level homes ................. 50

Figure 16: Simulated temperature profile of a test house for 10 days in January, 2013 .. 53

Figure 17: Actual temperature profile of HOME 01 for 10 days in January, 2013 ......... 54

Figure 18: Plot of HVAC consumption and average inside temperature against average outside temperature to find absolute preferred temperature .........................56

Figure 19: Example of the absolute preferred temperature estimation for HOME $01 \ldots . .56$ 
Figure 20: Example plot of deviation $\left(D_{t}\right)$ vs outside temperature $\left(T_{o}\right)$ for HOME $01 \ldots . .59$

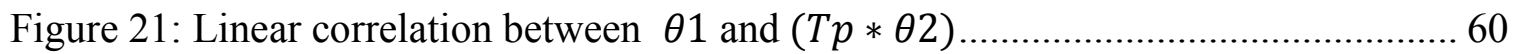

Figure 22: Simulation of room temperature (orange) based on outside temperature (green) for 10 days in January, 2013 at different levels of $\theta$ 61

Figure 23: Johnson SB distributions with means of 2.5, 5 and 7.5 used to define $k_{1}$ for MAUF of comfort favoring, neutral and cost favoring households............... 71

Figure 24: Different input factors that affect the consumption of NTL and TCL ........... 74

Figure 25: A framework of using multi-attribute utility function for the simulation of NTL energy consumption scheduling ................................................. 81

Figure 26: Utility maximizing algorithm embedded in the Household entity ................. 83

Figure 27: A framework of using multi-attribute utility function to optimize HVAC load for the simulation of TCL consumption................................................ 92

Figure 28: Basic principle of model predictive control (MPC) .................................. 96

Figure 29: Outline of the model predictive control (MPC) mechanism used to simulate utility driven TCL consumption behavior................................................ 98

Figure 30: Sub-metered appliances and temperature data for HOME 01 for $2014 \ldots \ldots . .102$

Figure 31: Histogram plots for $\lambda$ and $\gamma$ estimation for heating cycle .......................... 104

Figure 32: Histogram plots for $\lambda$ and $\gamma$ estimation for cooling cycle ......................... 105

Figure 33: Three period graphical representation of the optimization problem ............. 110

Figure 34: Screenshot of system load information provided by GEPB on their website 116

Figure 35: Illustration of various states for the Markov chain model........................... 117

Figure 36: Utility functions and average values of $\mathrm{X}$ and $\mathrm{Y}$ for (a) Cost favoring / Risk neutral, (b) Cost favoring / Risk Averse, (c) Comfort favoring / Risk neutral and (d) Comfort favoring / Risk averse test houses.

Figure 37: Average daily consumption profiles of washer load by 26 test homes in 2013

Figure 38: Average daily consumption profiles of dryer loads by 26 test homes in 2013

Figure 39: Average daily consumption profiles of dishwasher by 26 test homes in 2013 
Figure 40: Load validation of TCL model using GEPB system load for 2013 129

Figure 41: Static variable pricing structures: Flat rate, TOU abrupt, TOU gradual ....... 131

Figure 42: Hourly load profiles for the three appliances .......................................... 138

Figure 43: Hourly total load profiles for NTL model .............................................. 138

Figure 44: Example calculation of probabilities in PTable from CTable ..................... 142

Figure 45: Simulation of electricity rates over time based on RTP ............................ 144

Figure 46: Temperature response for a low income (cost-favoring) household ............. 150

Figure 47: AC command signals for a low income (cost-favoring) household .............. 150

Figure 48: Temperature response for a medium income household .......................... 151

Figure 49: Temperature response for a high income (comfort-favoring) household...... 152

Figure 50: Average hourly load profile under different pricing structures................... 154

Figure 51: Comparison of static variable pricing models showing average daily load, monthly bill, coincident load and coincident load factor for the population

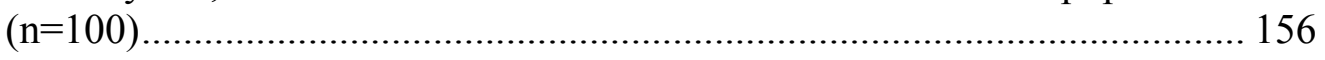

Figure 52: Simulation of rates under various RTP models for the first 10 days ........... 159

Figure 53: Energy consumption of HVAC by (a) Group A, (b) Group B and (c) Group C

Figure 54: Average room temperatures under various pricing structures for (a) Group A, (b) Group B and (c) Group C 161

Figure 55: Steady state probability that the system will peak over time. 164

Figure 56: Comparison of the demand charge based dynamic variable pricing models showing the population response of average daily load, monthly bill, coincident load and coincident load factor $(\mathrm{n}=100)$

Figure 57: The steady state probabilities of the system being at peak state under various demand charge based dynamic rates 167

Figure 58: Macro MPC for optimization of real-time price …................................. 176

Figure 59: Reference load matching via macro-MPC model 177 


\section{CHAPTER I}

\section{INTRODUCTION}

Facing the urgency of conserving global energy resources and controlling global climate change, governments around the world have placed energy efficiency as their top priority. The limited supply of natural resources coupled with growing population and mass urbanization poses a real threat to sustainability in our society. As we continue to debate the possible repercussions of energy crisis to the future of our society, we cannot overlook the exceeding energy demand at present mostly attributed to rapid urbanization, technological development and over-population. There has definitely been a surge in responses in the form of global policies promoting renewable energy, energy efficient products, reducing greenhouse gases, addressing the carbon footprint of society, etc. Although long term solutions are an important aspect of the energy solution, it is as important to address energy conservation and optimization in the existing system.

The Annual Energy Outlook 2015 report from the US Energy Information Administration (EIA) indicated that the total energy consumption in the US in 2014 was around 98.32 quadrillion Btu ( Figure 1), 21.53 quadrillion Btu of which was consumed in the residential sector. The total energy consumption in the residential sector has seen about $30 \%$ increase from 1990 to 2011, an indication of a consistently increasing demand. Energy independence as part of the solution to energy efficiency has become a pressing issue for the society. 


\section{U.S. Energy Flow, 2014}

(Quadrillion Btu)

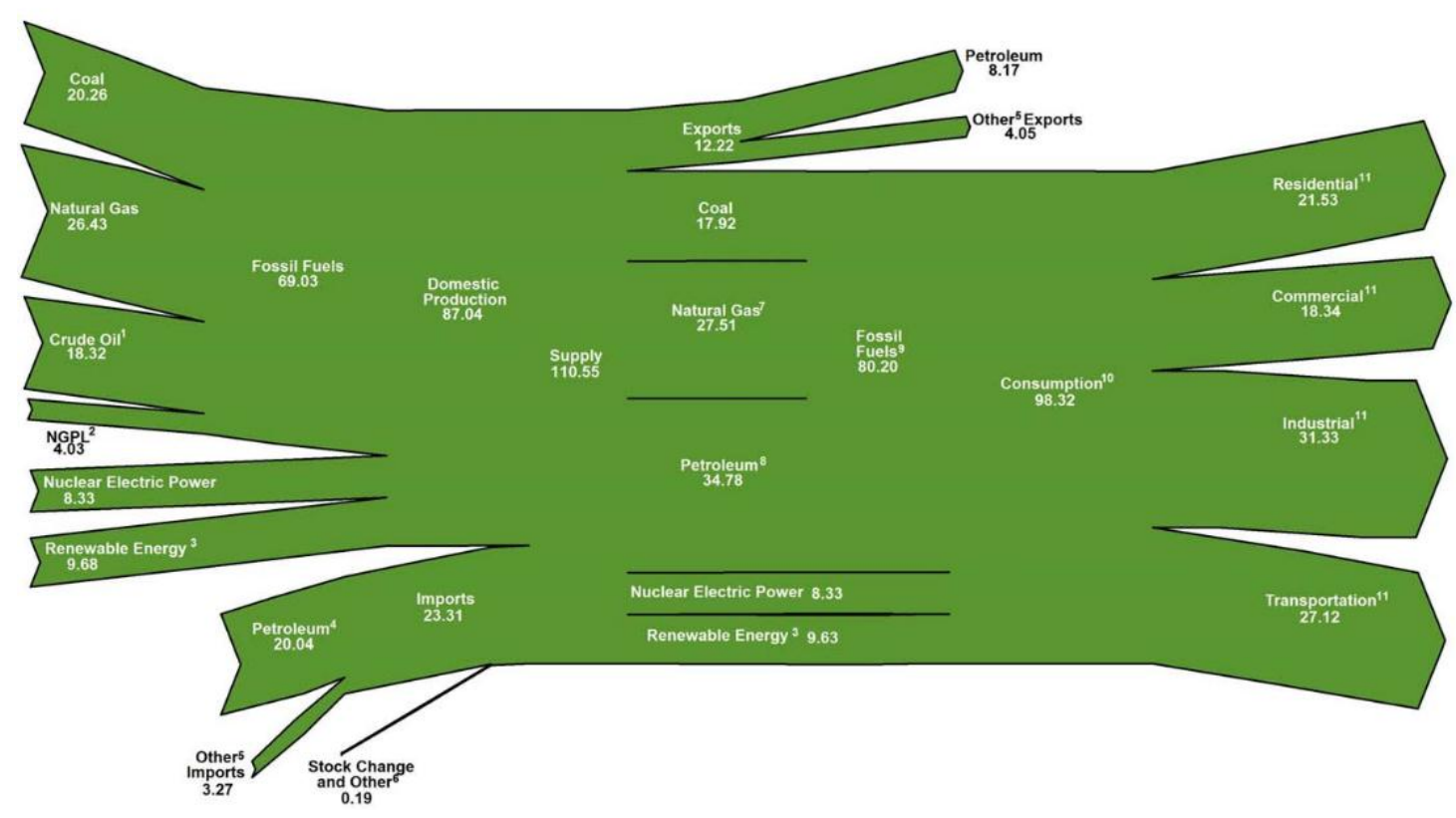

Figure 1: Total US energy flow, 2014 (EIA, Annual Energy Review, 2015)

The motivation for this dissertation can be established upon the need for energy efficiency in the residential electricity market. We use three key concepts to support our study: demand response (DR), distributed load control and innovative pricing models. These topics are discussed in the subsequent sections in this chapter. First, we discuss the impact of residential sector energy usage on the electricity distribution system and background of demand response. Secondly we explain various methods of implementing DR from the perspective of the authority of load control. The importance of studying consumer behavior is linked with the impact of distributed effect of DR. Thirdly, we briefly discuss various innovative pricing models used to promote efficient residential electricity consumption and setup the motivation to study various pricing mechanisms. 


\subsection{DEMAND RESPONSE IN RESIDENTIAL SECTOR}

The residential sector holds a significant share of the total energy usage, almost $50 \%$ of which can be attributed to electricity (Figure 2). In 2013 the share of total electricity use in the residential sector was $37.7 \%$, while commercial, industrial and transportation sector had 36.3\%, 25.9\% and $0.2 \%$ shares, respectively (Figure 3). A significant portion of electricity usage is attributed to the residential sector, thus making it an influential area for the study of energy efficiency. The EIA predicts a further $17 \%$ increase in the primary energy consumption in the buildings sector by 2035, which further signifies the importance of residential sector in overall energy efficiency.

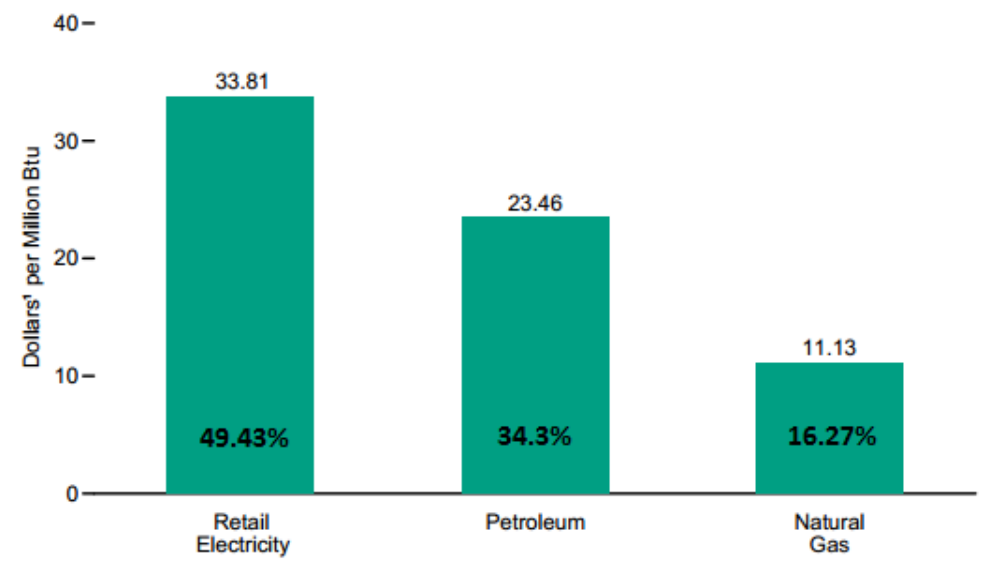

Figure 2: Distribution of energy in the residential sector (EIA, 2015)

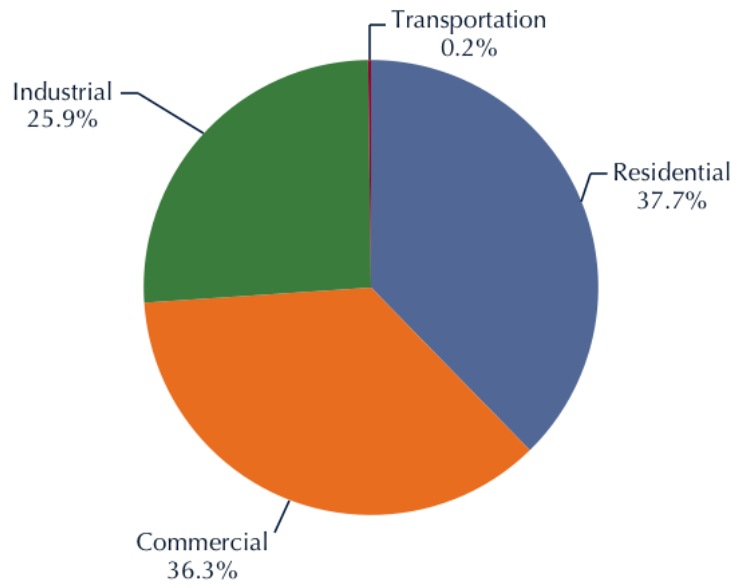

Figure 3: Electricity consumption by end-use sector share for 2013 (EIA, 2015) 
The consumption of electricity in residential sector can be influenced by providing various incentives to consumers. The change in load consumption pattern caused by these incentives will be of interest to us in this dissertation. The EIA indicated that out of the total electricity consumption in residential sector in $2014,13 \%$ was attributed to air conditioning loads and $30 \%$ was attributed to the remaining thermostatic loads (water heater, space heater and refrigerator) (Figure 4). Load shifting behavior can be targeted to change particular appliances based on flexibility of usage and their contribution to total energy consumption. Most literatures have been focused on energy scheduling of HVAC and water heater loads. In this dissertation, we not only study Thermostatically Controlled Loads (TCL) like HVAC but also some major Non Thermostatic Loads (NTL) loads with potential flexibility of usage (e.g., washer, dryer, dishwasher, etc.).

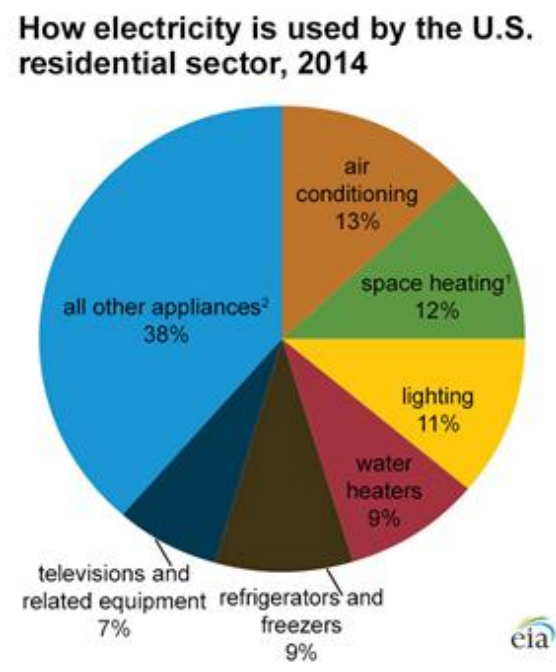

Figure 4: Distribution of electricity usage in a residential household (EIA, 2015)

In an attempt to alleviate the rising demand of electricity, many have proposed the curtailment of peak demand of residential electricity load. In general, the inherent supply 
process of the US electricity distribution system coupled with the uncertainty of demand caused by weather conditions, consumer behavior, grid failure, unexpected generator failures, etc. results in a highly unbalanced system. The electricity market operation relies greatly on supply and demand of energy and an accurate forecast of the demand is very crucial for the system. The use of electricity however can vary drastically within short time frames as a result of the consumer behavior, weather conditions, etc. Unbalanced load during peak and off-peak hours, and unforeseen system fluctuation are generally tackled using ancillary services. Operating generators below capacity with the ability to increase generation instantaneously when called for is called spinning reserves. Nonspinning reserves include fast-start generator such as gas turbines that can be brought online in a short time, as well as provisions to procure energy from other systems or retract energy that is being exported. Ancillary services are designed to protect the grid and ensure high reliability but result in power loss and are expensive to operate.

In power economics literature, demand response (DR) has long been proposed for incentivizing consumers to change their energy consumption behavior in achieving load leveling. Energy efficiency in a grid network can be achieved if the system load can be accurately predicted and balanced. DR offers various incentives to subscribers who would regulate their usage patterns. It tries to change the energy consumption behavior of consumers by providing them with financial incentives and education, thus affecting the demand for energy. Incentives are provided to encourage consumers to use less energy during peak hours and more energy during off-peak hours in an attempt to level the system load. Some contracts provide financial incentives in the form of rebates to consumers willing to reduce load at a requested time or a predetermined time slot. This 
type of an arrangement relies on the behavior of consumers and their marginal utility of energy reduction to achieve load control. Other contracts give subscribers the benefit of a reduced rate at all times except during peak periods when the rates are increased.

More recently, the development of smart grid has a prominent value for DR since data can be securely and easily communicated between the electricity consumer and the provider. This makes it easier for electricity providers to get load information from the consumers as well as to communicate the cost information in real time. Among other infrastructure, smart meters are crucial to enable DR programs by providing consumers with information such as pricing, energy consumption, and billing, and by facilitating two-way communications between utility and consumers. Furthermore, most DR programs provide financial incentives and assume that consumer behavior is driven primarily by cost. Fahrioglu et al. (2000) applied game theoretical principles to study the interaction between the utility company and its customers by designing incentive compatible contracts. Mohsenian-Rad et al. (2010) discussed the use of a distributed algorithm on smart meters to find optimal consumption schedules for subscribers. They achieved peak load reduction by using a pricing scheme based on non-linear cost functions. Samadi et al. (2010) proposed a real-time pricing algorithm based on utility maximization based on the amount of load used by the customer.

In a pilot program conducted by GE and LG\&E in 2009 on incentivizing consumers for using smart appliances that regulated energy use based on peak and offpeak loads, participants were highly satisfied due to their ability to override the system (Kentucky Public Service Commission, Case no. 2007-00117). According to the customers, being able to override the system, albeit at the loss of their incentive, was a 
key to avoid major lifestyle interruptions. This motivates our research of studying consumer behavior as a function of their perceived convenience or comfort as well as cost incentives, because it is unrealistic in assuming that all people value these incentives equally. In addition to this trade-off behavior it is also fair to say that consumer responses are distributed in nature owing to the fact that different people characteristically react to situations differently, hence requiring a distributed (i.e., decentralized) approach to modeling their response.

\subsection{CENTRALIZED AND DECENTRALIZED CONTROL}

The goal of achieving grid stability by altering load consumption is accomplished by one of two ways: centralized and decentralized control. Centralized control refers to the direct interference by the utility company to alter energy consumption of willing consumers in exchange for some form of incentive. Decentralized control leaves the actual load shifting responsibility on the residents based on their own response to financial incentives. Research in DR programs in the past have dealt with both methods in the form of different subscription based contracts or pricing mechanisms. Many utility companies offer some sort of DR program in an attempt to lower peak load consumption. The main difference between centralized and decentralized control lies in the authority of action. Direct load control for example, provides incentives to consumers who are willing to allow the utility company to take control of their energy units so that they may reduce consumption during high demand periods. Since the load reduction is controlled by a central control system, the desired effect is predictable and centralized. Decentralized control on the other hand relies on the control actions of individual users motivated by 
some form of incentives. The effect of DR in such cases is unpredictable and highly dependent on consumer behavior. Variable pricing mechanisms that charge differential rates at peak hours would in theory motivate consumers to reduce their consumption and shift it to off peak hours, but the overall impact on a system level is hard to predict due to the distributed and varying nature of consumer response.

When subject to decentralized programs, the utility company is not the only one at a disadvantage due to unpredictability. Some contracts are designed such that consumers agree to reduce energy during particular times called out by the utility, while facing a potential penalty if the agreed reduction is not achieved. Responding to complex DR rules and maximizing benefits becomes a daunting task for consumers and thus subscription to such programs are significantly reduced. Many intermediary agents, called curtailment service providers, have emerged to solve this problem by taking responsibility of load control on behalf of customers in order to receive maximum benefits from DR programs. This entails handing over remote access of some appliances, especially HVAC, to these agents. Special equipment with capability of remote control may be required for this purpose. Although, the authority of action is neither with the customer or the utility, this may be considered a partially centralized system. Some aggregators also provide energy audits and appliance upgrades to ensure full benefits of the programs are achieved.

With the development of smart meters and the advancement in smart grid networks, the prospect of centralized control is looking better for the utility companies. The control system requires a direct data and signal communication mechanism or wireless switches so that utility companies may have direct remote access to individual 
energy units. This gives more control to the utilities in achieving desired energy reduction. There are many ongoing researches that deal with optimization of network wide energy scheduling with direct load control. Most direct load control methods deal with thermostatic loads such as HVAC and water heating, since they compromise the majority of end use energy for residential homes (Figure 5).

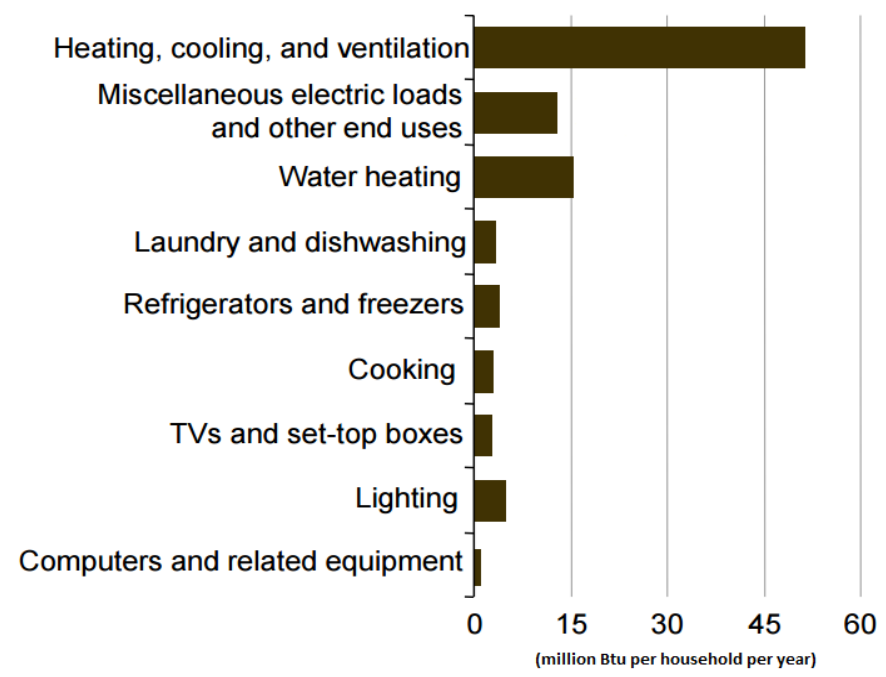

Figure 5: Residential sector delivered energy intensity for selected end uses (EIA, 2015)

As promising as it may seem, centralized control is limited by the amount of energy that can be controlled, limited by the number of subscribers to the program. It is not possible to say whether all residential consumers will agree to give utility companies total control of their energy usage. Independent of centralized programs, differential pricing mechanisms can still be implemented to promote voluntary load shifting behaviors. This makes the area of decentralized control a viable and long term option for system wide energy management. If consumer behavior can be appropriately modeled, different variable pricing mechanisms can be designed to provide attractive incentives, ensure consumer participation and achieve better demand response. 


\subsection{RESIDENTIAL LOAD CONTROL VIA INNOVATIVE PRICING MODELS}

Regulation of energy consumption in the residential sector has been dominated by using financial incentives. Incentives include contract signup rewards, discounted pricing, unit based rebates, variable pricing, etc. Although different states have tested and implemented various methods, differential pricing structures are by far the most prevalent. Pricing models can be designed with variable electricity rates for peak and offpeak times to motivate consumers to reduce peak consumption. This is simple to understand for consumers as well as easy to implement on a network level. Even after more than 50 years of modern electricity distribution, the billing system for residential consumers is still predominantly based on fixed unit price (flat rate) systems. As the need for demand side management grew in the past few decades, utility companies across the country started implementing new pricing structures, different from the flat rate models, in an effort to reduce energy losses and control consumption. These new pricing models, however, are only provided as an optional service to consumers who are willing to participate. Policy level decisions are needed to completely overhaul existing pricing methods and this usually takes time. Thus new pricing models can only be implemented as optional services.

Even without using variable rates, some utility companies use different pricing contracts for consumers to choose from. These contracts usually have different fixed unit prices per contract, variable block rates, fixed unit rate with a demand charge and other variations using fixed unit prices. Demand charge refers to the maximum energy drawn by a consumer in any hour during the billing period. Pricing structures that charge an extra amount for the maximum hourly power are designed to reduce system peak by 
motivating consumers to lower their maximum consumption. The assumption is that the system peak coincides with a majority of consumerse maximum load time period, thus including demand charge in an electricity rate promotes a system peak reduction.

Time-of-use (TOU) rates are a common pricing structure that provides variable rates depending on the hour of the day. Consumers who enroll for this billing scheme are provided with a predetermined time schedule for peak, off-peak and sometimes intermediate hours. A fixed rate for each of the time window is set, with peak hours having much higher rates than off-peak hours. The consumers are thus expected to shift higher load usage to off-peak hours to take advantage of lower cost. Since load consumption can be very different depending on the season, TOU rates that are designed for specific seasons are also implemented by some utilities. Similar to the underlying concept of TOU rates are pricing models like critical peak pricing (CPP) and peak time rebate (PTR). The time windows are not as blocked as for TOU and there are no off-peak periods. The standard rate is slightly reduced such that the consumer can enjoy lower costs at all times, except for a few time slots referred to as „events ${ }^{e e}$ called by the utility, during which the rates are set to be extremely high. While CPP charges a premium rate during these event periods, PTR gives credit to consumers who reduce their load during event periods in the form of rebates.

The design of pricing structure has a direct impact on the amount of load reduction. Although research in this area have resulted in many novel contract systems and pricing models proposed to achieve better peak reduction, the most prevalent variable pricing mechanisms in practice today are TOU, CPP, PTR, variable contracts, etc. These methods may be considered as static variable pricing because predetermined time slots 
are assigned fixed prices. The uncertainty of consumer behavior and weather changes is not addressed very well by these methods. Dynamic variable pricing methods are much better in matching weather and load fluctuations. If the system load unexpectedly increases due to unforeseen reasons, dynamic variable pricing may be able to increase rates accordingly, thus countering the fluctuation.

A handful of utilities currently provide real time pricing (RTP) by forwarding the real-time spot market rate directly to the consumer, thus removing any liability on their part. Even though this does not allow utilities to mark up the rates for a profit margin, they are reducing cost on their side as well by placing the responsibility of high spot market prices directly on the consumer. Some energy providers are also experimenting with hybrid models like variable peak pricing (VPP), where peak and off-peak hours are predefined similar to TOU, but the electricity rates during these periods vary according to market conditions. Innovative pricing models that provide dynamic variable pricing can prove to be very beneficial in decentralized load control, and there is a lot of room for research and development.

\subsection{CONTRIBUTIONS OF THE DISSERTATION}

The primary goal of our research is to study consumers"e behavior in response to DR programs and to build a simulation platform that can be used to test various pricing models. Using decision analysis methods, the consumer response to incentive based energy consumption is modeled as an economic utility maximization problem with the social theory of human rationality. The expectation of load shift from peak to off-peak hours is mainly dependent on two factors: the cost incentives or savings obtained from an 
energy consumption schedule and the resulting comfort or discomfort experienced by consumers. We take into account the fact that consumers vary in the tradeoffs that they are willing to make between cost and convenience/comfort.

An object oriented simulation model is developed to study the end-user response of a typical household. By simulating a population with diverse utility functions, we are able to simulate close to true system response in terms of load usage. Also, since the simulation is defined from a bottom up approach with individually defined appliances as well as specifically designed algorithms for consumers to react to thermostatic and nonthermostatic loads, we have the freedom to model all types of load consumption if required. A validated model can then be used as an experimentation tool to conduct sensitivity analysis between different pricing models and optimize consumption behavior via optimal pricing mechanisms.

Model Predictive Control is a method of system control under uncertainty. We use this concept to model the behavior of consumers by optimizing their utility function in the presence of other constraints. We also study a novel dynamic pricing model using coincident demand charge. A conditional Markov chain is used to model the uncertainty of peak to off-peak transitions in a system. Consumer behavior modeling is one of the key components of this research, which allows us to study interactions between the consumers and utility providers. The obtained results allow us to understand the response of consumers effectively and take advantage of dynamic pricing to increase system wide energy efficiency. As such, this method can be instrumental in facilitating an effective Demand Response framework, which enables the utility providers to adopt efficient demand management policies. 
The remainder of this dissertation is organized in the following way. A literature review of relevant research work in the field of energy systems and demand respond is presented in Chapter II. Chapter III discusses the consumer behavior modeling methods, explaining the classification of a population based on various parameters and the development of multi-attribute utility functions. Chapter IV presents the methodologies used in developing energy consumption models. This includes the models for nonthermostatic loads (NTL) and thermostatically controlled loads (TCL) along with the simulation model, model predictive control, multi-period optimization techniques and peak forecasting using conditional Markov chains. The model validation, along with sensitivity analyses on various pricing models are discussed in Chapter V, after which conclusive remarks and direction for future work in provided in Chapter VI. 


\section{CHAPTER II}

\section{LITERATURE REVIEW}

In light of increasing energy crisis with frequent power curtailment and scheduled blackouts during peak demand periods, regulating the use of energy has become critical for the government as well as utility. The need for higher energy efficiency has not only become a business goal for power companies but also a social issue as part of the solution to energy independence. In this chapter we will explore the literature in the field of demand response (DR) and electricity consumption behavior. The rest of the chapter is divided into separate sections discussing various specific areas. In particular Section 2.1 reviews literature about DR and research in smart grid applications, Section 2.2 discusses consumer behavior and decision theory in the field of energy consumption, Section 2.3 explores work done on control of thermostatic loads using model predictive control (MPC), Section 2.4 reviews existing DR practices and current state of residential billing systems, and finally Section 2.5 reviews agent based simulation models in energy application.

\subsection{DEMAND RESPONSE IN SMART GRID}

In the literature, there have been quite a few methods of modeling the energy distribution in the residential sector. A detailed approach to residential energy resource modeling was presented by Guttromson et al. (2003) where the individual characteristics 
of major appliances and human behavior patterns were preserved. Two types of bottom up approach were presented: empirical and deterministic. While the empirical model was based on measurement and probabilities of clustered load at a feeder level, the deterministic model gave a detailed systems model of individual appliances. Shimoda et al. $(2004,2007)$ developed a model that simulates city-scale energy consumption in the residential sector by considering different types of households and their energy behavior. This kind of a residential end-use model allowed them to study the effects of various policies and energy saving measures such as heat insulation, higher efficiency air conditioners, daylight saving time, etc. The validity of such residential energy consumption models plays a vital role in the analysis of Demand Response (DR).

Demand Response (DR) is defined as the change in electric usage by end-use customers from their normal consumption patterns in response to changes in the price of electricity over time. They incentivize lower electricity usage during peak hours when the market price is very high. Variable contracts have been used to control energy usage of industrial and commercial consumers for a long time, but its application to residential consumers has also gained much interest in recent years. It is expected that Demand Response programs will be designed to decrease electricity consumption or shift it from on-peak to off-peak periods depending on consumers ${ }^{\text {ee }}$ preferences and lifestyles. Although DR can be used as a direct load control system, it is mostly a voluntarily action taken by a consumer to adjust the amount or timing of his or her energy consumption.

The purpose of DR being the implied change in consumption pattern of end-use customers, it can be implemented in several ways such as incentive contracts and pricing 
schemes. There have been many studies in the literature in this area. Fahrioglu and Alvarado (2000) adopted mechanism design with revelation principle from game theory to design incentive based curtailment programs. Samadi et al. (2010) proposed a real-time pricing mechanism facilitated by an energy consumption controller (ECC) that found the optimal energy consumption for each consumer to maximize aggregate utility of the system. Mohsenian-Rad et al. (2010) also used non-linear cost functions and game theory method to find optimal consumption schedules for subscribers by running a distributed algorithm on smart meters. Both incentive based contract designs and pricing designs have been topics of research in this area.

The advent of smart grid has fueled the already prevalent interest in DR. With the communication system implemented through smart grid, transfer of information becomes very easy, thus making DR even more pertinent. Rahimi and Ipakchi (2010) explain the challenges and solution to implement DR in a smart grid environment. Fan (2010) studied Demand Response on a distributed framework. He applied congestion pricing to the DR problem to show that individual users adapt to price signals to maximize their own benefit, thus taking the burden of load leveling. The DR techniques studied by Mohsenian-Rad et al. (2010) are also primarily based on the foundation of smart grid. They proposed an optimal and automatic residential energy consumption scheduling framework which aimed to achieve a trade-off between minimizing the payment and the waiting time for the operation of each household appliance based on the needs declared by users. As such, the nature of end users to maximize their own benefits in response to changing price structures have been taken into consideration, but a quantifiable measure of convenience is often left out. Kondoh (2009) also considered end-user comfort in his 
direct load management scheme with two way communication by allowing each consumer to maintain a desired set point while the air conditioner tried to maintain the temperature within a certain limit of that set point. This however does not provide any information regarding household characteristics in their trade-off willingness.

The increasing penetration of electric vehicles (PEV) and the demand for electricity for charging them also plays a significant role in the study of DR. Vandeal et al. (2010) achieved peak load reduction by controlling the charging of PEVs in smart grid, using a multi-agent solution instead of a quadratic program scheduler. In the study of direct load management while considering end-user comfort, Kondoh (2009) considered $\mathrm{PEV}^{\mathrm{ee}} \mathrm{s}$ as a major controllable load. On a commercial scale Kulvanitchaiyanunt et al. (2013) studied the control of charging stations for PEV's in order to minimize total cost of buying electricity as well as implementing different policies to help balance the system load.

\subsection{MODELS FOR DECISION MAKING IN ENERGY USE}

Modeling consumer behavior in terms of energy usage has always employed utility maximization. Many literature in energy economics deal with models that maximize expected utility based on some variables to describe the decision making process of consumers. However, the majority of work done in this area considers that the utility function of consumers depend on energy costs or financial tradeoff in some form. The notion of convenience maximization for consumers has been discussed intermittently recently. Avci et al. (2013) use a discomfort tolerance index to represent various consumer attitudes in choosing the thermostat set point based on varying prices. The 
objective function of their Model Predictive Control (MPC) model includes a weighting coefficient given to the discomfort minimization part. Xu and Deng (2013) use an exponential utility function to model consumer choice between different contracts. Various parameters such as estimated savings, utility bill and set point control limit affect the utility function, which is considered a convex function. However the interaction between the utility of convenience and utility of cost is ignored. As discussed by Castilla et al. (2011), predictive mean vote (PMV) is a common method for evaluating thermal comfort index for a large group of people in certain environments. PMV is used for temperature control of large public spaces such as office, supermarket, mall, etc. Li et al. (2011) study demand response using utility maximization. Both the utility company and customer have their own objective functions and the equilibrium of both objectives is desired. The consumer"s objective consists of utility functions for each appliance based on the amount of power it consumes at each time interval.

As most economics literature point out, the decision making behavior of a person can be modeled using utility functions. By assuming varying marginal utilities for any commodity over its amount, non-linear functions are widely used to denote the value of any product to the decision maker. When dealing with utility of money, the concept of diminishing marginal utility is well established. Most economists use the term utility to denote the satisfaction gained from acquiring a particular commodity, in this case money. The law of diminishing marginal utility states that the marginal utility, or increase in satisfaction due to the addition of a single unit of the product, decreases as the total amount of the product becomes greater. This simply means that the more money you have, the lower the value of the next dollar. The implication of this theory is that, the 
utility function of money is always concave, or convex when dealing with utility of cost. Thus the varying behavior of different people with different risk natures must be represented by the convexity of the utility function alone.

The decision making ability of humans is limited by the capacity of information processing, as famously stated by Herbert Simon"s theory of rationality. Simon (1955) claimed that a person is only capable of making decisions based on one"s limited cognitive capacity. If a person is given a large number of options to choose from, he or she will likely make a poorer decision as compared to a person given only a few choices. This has a significant impact on the way we model consumer behavior, since we cannot assume a perfectly optimal decision is made at all times. Many literature that rely on utility maximization as a decision making behavior seem to overlook the uncertainty of environment and rational behavior and assume perfect information is available for making optimal decisions.

\subsection{THERMAL MODELING AND MODEL PREDICTIVE CONTROL}

Among various types of electricity consumers, a significant portion of total energy usage is from residential consumers. The Annual Energy Outlook 2015 report from the US Energy Information Administration (EIA) indicated that residential customers contributed about $21.53 \%$ of the total energy used in 2014 . This signifies the importance of changing energy consumption behavior in residential customers in order to increase energy efficiency. Thermostatically controlled loads (TCL) make up about $43 \%$ of the total residential energy use; $13 \%$ is attributed to air conditioning alone (EIA, Annual Energy Outlook 2015). Therefore, we focus mainly on the consumer behavior of 
using AC, and the research may be extended in the future to include other TCLs as well. Non-thermostatic loads (NTL) such as clothes washer, dryer and dishwasher are also studied as appliances with flexible usage.

We use model predictive control (MPC) to calculate thermal dynamics in conjunction with optimization. In the literature, MPC is a method of system control that is gaining much popularity in recent years for modeling thermostatic loads. MPC determines appropriate control actions, at every sampling step, by optimizing the control objective over a finite time horizon. The decision made by an MPC model is usually based on the evolving predictions of stochastic variables that affect the desired output. In the literature, Vasak et al. (2011) used MPC to model the temperature control of a house by using the least possible energy. Xu and Deng (2013) studied a game theoretical model for optimizing incentive contracts, where TCLs are supplied by intermittent renewable sources such as wind power, and TCLs operations are simulated through MPC. They consider TCLs as flexible load and use incentive based contracts, where the savings of each contract is dependent on the number of subscribers. Avci et al. (2013) used MPC to control HVAC load under dynamic real-time pricing. Temperature set points were made variable and dependent on the day-ahead prices.

\subsection{RESIDENTIAL ELECTRICITY RATES AND DR PRACTICES}

The way residential consumers are charged for electricity hasn"t changed that much ever since the modern electricity distribution system came into operation in the

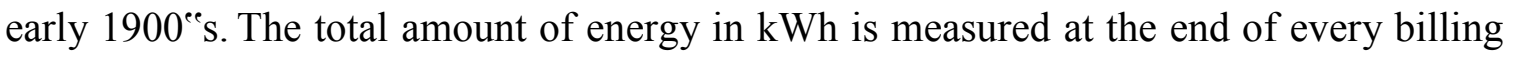
cycle (typically a month), and a fixed unit cost per $\mathrm{kWh}$ is applied to this amount. This 
has been the norms of electric billing systems since the beginning, and it is the most prevalent form of billing method even today. The steady decline of natural resources and over population has led to a shift in the balance of energy economics. As the issue of global energy crisis becomes increasingly urgent, the importance of grid stability and energy efficiency grows too. The cost of peak power production as well as loss of unused off-peak energy has a much greater impact in energy economics today than before. Much interest has been generated in demand side management (DSM) in the past few decades to control load imbalance and improve grid stability by providing incentives to the consumers willing to change their demand. Various pricing structures with variable rates, both static and dynamic, have been studied and proposed as demand response (DR) programs. However, DR programs are only provided as an optional service and most utilities still enroll customers under flat rate billing structure by default. A brief review of existing electricity rates and DR programs are reviewed here.

The national electric grid in the United States is a vast network of generators, substations, transmission lines and distribution feeders. The power regulation is done by various independent system operators (ISO) or regional transmission organizations (RTO) based on the designated service areas. The distribution level authority for smaller regions lies on service providers or the utility companies. There are different types of service providers in different states and regions. Most cities operate under single providers such as ComEd (Chicago), San Diego Gas and Electric and Louisville Gas and Electric where residents are only served by a single entity. Some municipality services are also present where residents living in a particular municipality are served only by the municipality operated utilities. For example the city of Los Angeles is serviced by the 
Los Angeles Department of Water and Power (LADWP). However, in some states like Texas, consumers are able to choose from multiple utility companies to buy power from, for example TXU, First Choice, Green Mountain, Reliant, etc. This is called deregulated multiple competing providers, and competing utilities try to offer attractive pricing schemes to enroll consumers.

The standard residential electric rates vary between $5 \notin / \mathrm{kWh}$ to $15 \notin / \mathrm{kWh}$ depending on the service territory (Figure 6). Most electric utilities have a standard flat rate for all customers. Some utilities like LADWP, however, provide variable unit charges depending on different contracts. Although base charges, transmission charges, etc. vary along with the contract, the main purpose is to enroll different customer types based on their usage pattern to different contracts. The unit rates for these contracts vary between $13.92 \notin$ and $21.63 \notin$ per kWh (www.ladwp.com). Other companies make use of variable block charges where different rates are charged depending on the total amount of energy used in a billing period. For example, Austin Energy (Austin, TX) provides standard rates of $3.3 \phi / \mathrm{kwh}$ for the first $500 \mathrm{kWh}, 8 \not / \mathrm{kWh}$ if total energy is $501-1000$ $\mathrm{kWh}, 9.1 ф / \mathrm{kWh}$ if total energy is $1001-1500 \mathrm{kWh}, 11 \phi / \mathrm{kWh}$ if total energy is $1501-$ $2500 \mathrm{kWh}$, and $11.4 \phi / \mathrm{kWh}$ for consumption of greater than $2500 \mathrm{kWh}$ for summer period (www.austinenergy.com). This is a blocked system of fixed rates, which tries to motivate consumers to lower their total consumption.

Some utility companies also use a demand charge on top of the regular standard rate in order to encourage peak load reduction. CPS Energy in San Antonio, TX for example charges a peak capacity charge of $1.98 \not / \mathrm{kWh}$ for all consumption greater than $600 \mathrm{kWh}$ on top of the standard $6.91 \mathrm{k} / \mathrm{kWh}$ for total energy consumed 
(www.cpsenergy.com). A different approach to demand charge that penalizes peak power levels instead of energy consumption is also used. Intermountain Rural Electric in Denver, CO charges consumers with $\$ 6.94 / \mathrm{kW}$ on-peak and $\$ 3.56 / \mathrm{kW}$ off-peak demand charges in addition to a standard rate of $6.47 \mathrm{\phi} / \mathrm{kWh}$ (www.irea.coop). The demand charge is applied to the maximum energy drawn in any hour within the pre-determined on-peak and off-peak windows.

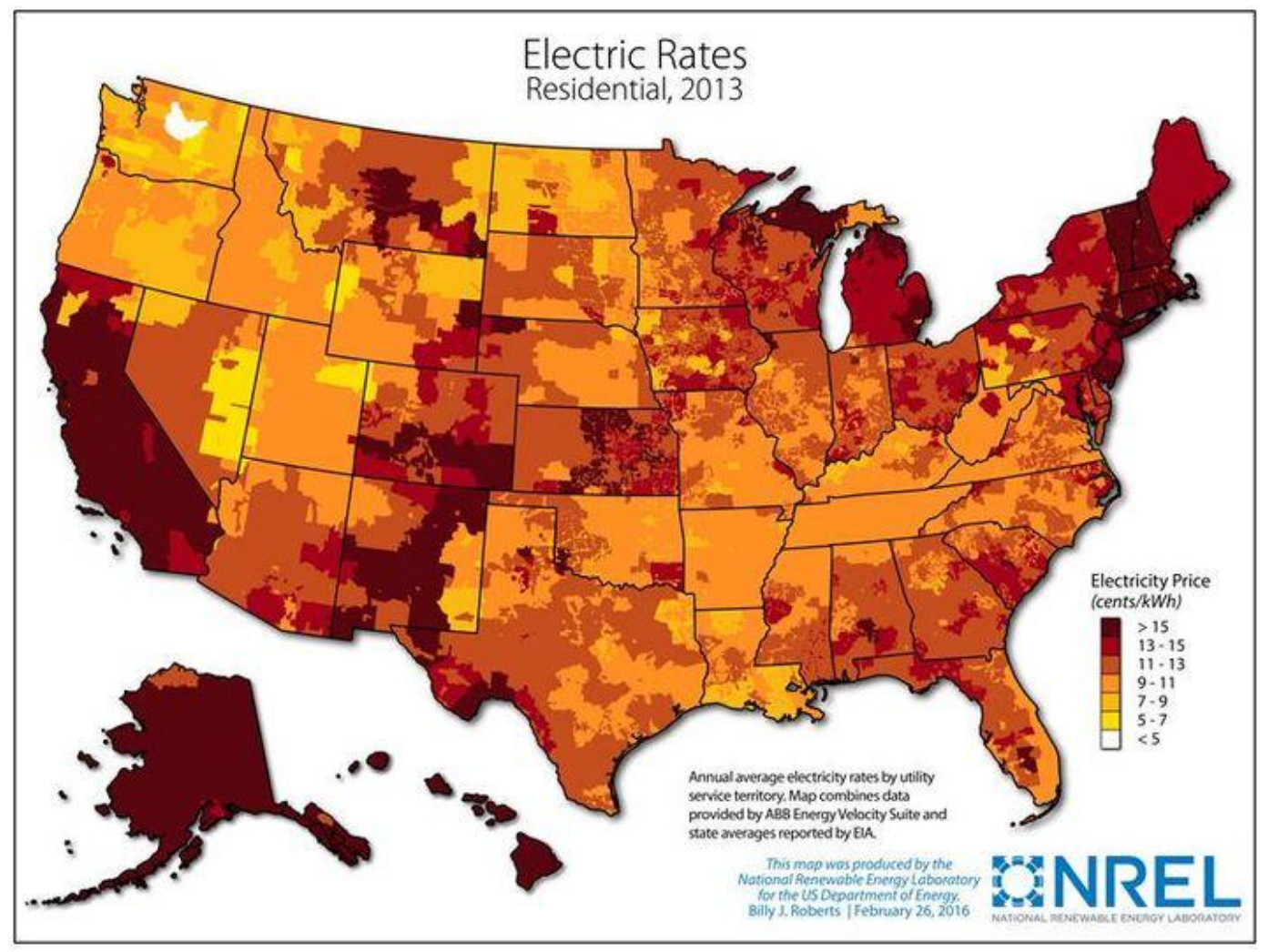

Figure 6: Residential electric rates by service territory in 2013 (NREL, 2016)

Aside from cost incentives using standard fixed rates, there are variable pricing mechanisms that are also used as optional DR programs. Variable pricing mechanisms can be divided into two categories: static and dynamic variable rates. The most widely used pricing structure with variable rates is the time of use (TOU) rates. Many utilities 
provide their customers a rate structure in which different times of the day are designated with different rates. Hours of maximum expected consumption are usually designated as peak hours and a higher rate assigned. Similarly, hours of minimum expected consumption are designated as off-peak hours and a much lower rate is provided. This encourages consumers to shift their load to off-peak hours and benefit from the lower cost. A variation of the TOU rates is the seasonal TOU, where summer and winter period are assigned either different rates or different on/off peak windows. Some utilities also attach demand charge on top of TOU rates. For example Duke Energy in Charlotte, NC offers a rate structure with $6.93 \notin / \mathrm{kWh}$ peak and $5.7 £ / \mathrm{kWh}$ off-peak rates. In addition to that, a $\$ 7.77 / \mathrm{kW}$ for summer and $\$ 3.88 / \mathrm{kW}$ for winter demand charge is applied to the peak load (www.duke-energy.com).

In contrast to static variable pricing, dynamic variable pricing attempts to change unit rates dynamically throughout the day based on various factors. These factors may include real-time fluctuation of market prices, variation of weather, fluctuation of total demand, etc. Although, true price of energy can be charged to the consumer by using this type of mechanism, it is not widely used right now due to the complexity in understanding stochastic behavior of consumers in response to such price fluctuations. Pricing methods like real time pricing (RTP) and critical peak pricing (CPP) could be considered as dynamic variable pricing structures. Customers of ComEd in Chicago, IL are provided with an option of subscribing to Residential Real Time Pricing (RRTP) (www.comed.com). RRTP program participants agree to pay the wholesale real-time market price, which is usually much lower except during peak hours. Participants have access to hourly prices online and also receive high price notifications and alerts. A 
slightly different method of real time pricing was tested by AEP Columbus Southern Power (Columbus, $\mathrm{OH}$ ) in 2014 as a pilot study (www.aep.com). This mechanism used a double auction system with real time pricing data. CPP subscribers on the other hand enjoy lower rates that the standard rate in exchange for extremely high rates during a few peak hours during a month. These peak hours are not known in advance and thus we can consider this model as dynamic. When the utility predicts a high demand, it notifies the customer of this period, in hopes that they will reduce their energy draw to avoid high cost. Pacific Gas and Electric (San Francisco, CA) and Arizona Public Service (Phoenix, AZ) are a few companies that provide CPP.

The current state of DR practices clearly indicates an opportunity of research in dynamic variable pricing mechanisms. Out of the 25 largest cities in the US, only two offer RTP and six offer CPP, however 21 of them have programs for TOU. The study of consumer behavior to dynamic pricing models will surely help in developing better DR programs. In this dissertation, we will study existing pricing structures as well as some innovative dynamic pricing mechanism to find optimal methods for peak reduction.

\subsection{SIMULATION OF ENERGY SYSTEMS}

Agent-based modeling and simulation (ABMS) is an approach for simulating the actions and interactions of autonomous agents, thus providing a framework to study or model adaptive environments. Credit for developing the first ABS that considers people as agents goes to Thomas Schelling. His model was an extreme abstraction of people and their interaction in a social system. Furthermore, it opened a new way to model one of the most sophisticated systems, namely those involving social processes (North and Macal, 
2007). The applications of this methodology range from modeling the growth and decline of ancient civilizations to agent behavior in stock market and supply chains. In agentbased simulation, an agent has a set of attributes and behavioral characteristics, which define how the agent behaves and how it interacts with other agents. As an example, an individual end-use customer can be an agent having attributes such as utility function, risk behavior, annual income, temperature preference, etc. Various rules or heuristic actions can be defined for the agents to use in certain conditions. These agents are then allowed to interact with the system and/or other agents to behave in an adaptive learning environment.

The amount of literature available on agent-based simulation methods is very high as it has a wide range of applications to model systems involving decision making entities. The electricity distribution system is no exception either, and the nature of the system is such that there exist independent decision making entities that interact with each other, for example end-use customer, utility company, generating unit, storage and distribution units, renewable energy units, etc. Azar and Menassa (2011) have used ABS to model the effect of energy policies on the behavior of building occupants and how they would affect building energy use. The building inter-occupant network and peer induced energy conservation behavior was also studied by Chen et al. (2011). In their model, they assigned an algorithm to each occupant to compare their energy usage with other occupants and update their energy decisions accordingly. In a community environment such as an apartment building the relationship between peer network and energy conservation is an interesting problem to be studied via simulation, since the generalization of human behavior based on few experiments is not sufficient. 
Molderink et al. (2009) and Bakker et al. (2010) developed an object oriented simulation model of a house in $\mathrm{C}++$ to study the impact of different combinations of micro-generators, energy buffers, appliances and control algorithms on the energy efficiency of the system. This was developed as an adaptable tool for the analysis of various combinations and scenarios within the system. Such an approach could be easily used, where an individual household is represented as an active agent that reacts to its simulation environment, the distribution network. Further, Zhou et al. (2009) studied the energy system on a much larger scale by implementing ABS to model the electricity market. Generator agents were embedded with algorithms to bid strategically in a deregulated electricity market. 


\section{CHAPTER III}

\section{CONSUMER BEHAVIOR MODELING}

Regardless of the type of DR programs, the decentralized effects due to the change in consumers ${ }^{e e}$ energy usage pattern are of key importance. Consumers are expected to change their load consumption pattern in response to a variable pricing model. Incentivized contracts provide direct financial rebates in exchange for load shifts whereas indirect cost advantage may also be provided by setting up specific pricing tariffs. In the latter case, customers have direct control over how much cost benefits they want to obtain or how much cost penalties they incur by regulating their energy usage by themselves. This also gives the customers complete control over the change in lifestyle brought about by this demand response pricing method (instead of rebate). Even under centralized control programs such as direct load control (DLC) or through intermediary agents, there are always provisions for the customer to override any control actions. As discussed earlier, participants in a pilot program conducted by GE and LG\&E in 2009 showed satisfaction towards the program due to their ability to override the control when desired (Kentucky Public Service Commission, Case no. 2007-00117). Hence, a perfect centralized control system seems unrealistic and supports the need to understand consumer behavior to study decentralized effects of DR programs.

We consider such cases and study the energy consumption behavior in response to various pricing structures. The behaviors of different people differ from one another due 
to individual preferences and perceived benefits. We model the energy consumption behavior as a decision analysis process using a multi attribute utility functions. We deal with two primary attributes central to the decision process, i.e. the monetary cost of energy usage and the cost of discomfort/inconvenience. In this chapter we describe the method in which consumers are categorized and modeled to reflect a diverse population response.

\subsection{CLASSIFICATION OF HOUSEHOLDS}

Population diversity is an essential part in the study of consumer response. It is necessary to differentiate groups of consumers with similar characteristics so as to represent distributed responses. There are many ways to categorize general households such as by race, education, economic status, family status, number of occupants, etc. Such difference in characteristics results in a varied load consumption pattern. We are interested in classifying households in such a way that their decision making process in response to DR may differ from one another. In response to the incentives provided by the utility company, different households will value the cost savings differently based on the inconvenience/discomfort they experience due to shifting their energy usage patterns. This variability must be reflected in the classification method.

We first classify the population based on the socio-economic status of households by considering average annual household income as the criteria. We assume that people with high income will value the convenience more than cost (low utility for cost), whereas a low income family will do the opposite and value cost more than convenience (high utility for cost). The distribution of average annual income for 2010 and 2014 is 
shown in the histogram in Figure 7 based on the US Census Bureau data (www.census.gov). We can see a left skewed distribution with an approximate mode of $\$ 30000$ - \$35000. The assumption that high income households have a lower utility for cost than low income households must reasonably be reflected in the amount of energy consumed. If a consumer does not care too much about the cost of energy, then the amount of consumption should also be higher compared to a consumer who is sensitive to cost. The total annual energy consumption for 1200 homes in Glasgow, KY for 2014 was analyzed. The histogram for this data is shown in Figure 8. Similar to the distribution of homes based on annual income, the distribution of annual energy consumption is also skewed to the left. Although the sample sizes are different, a general matching trend can be observed in both graphs, partially supporting our assumption.

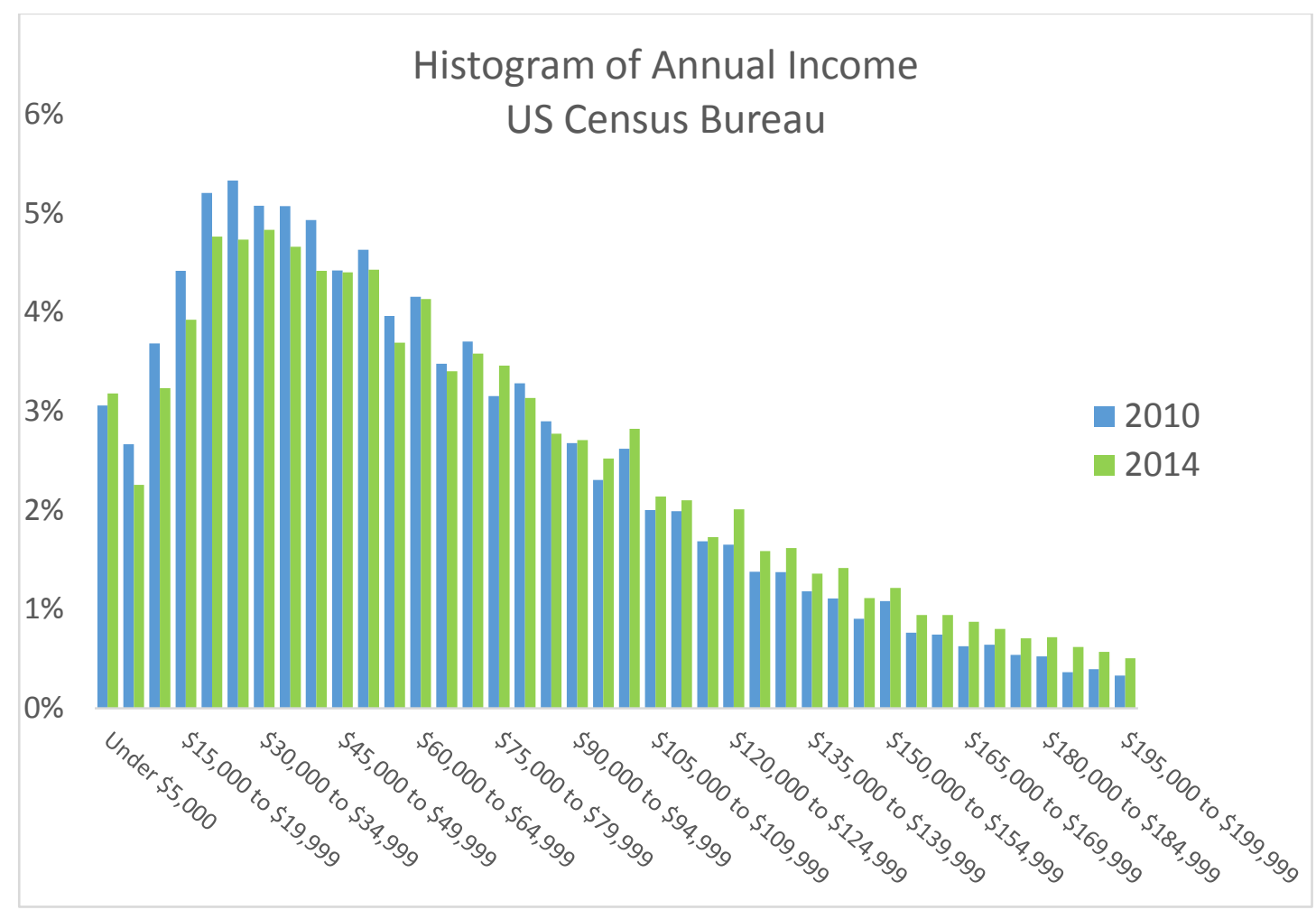

Figure 7: Histogram of average household annual income for 2010 and 2014 


\section{Histogram of Est. Annual Energy Consumption}

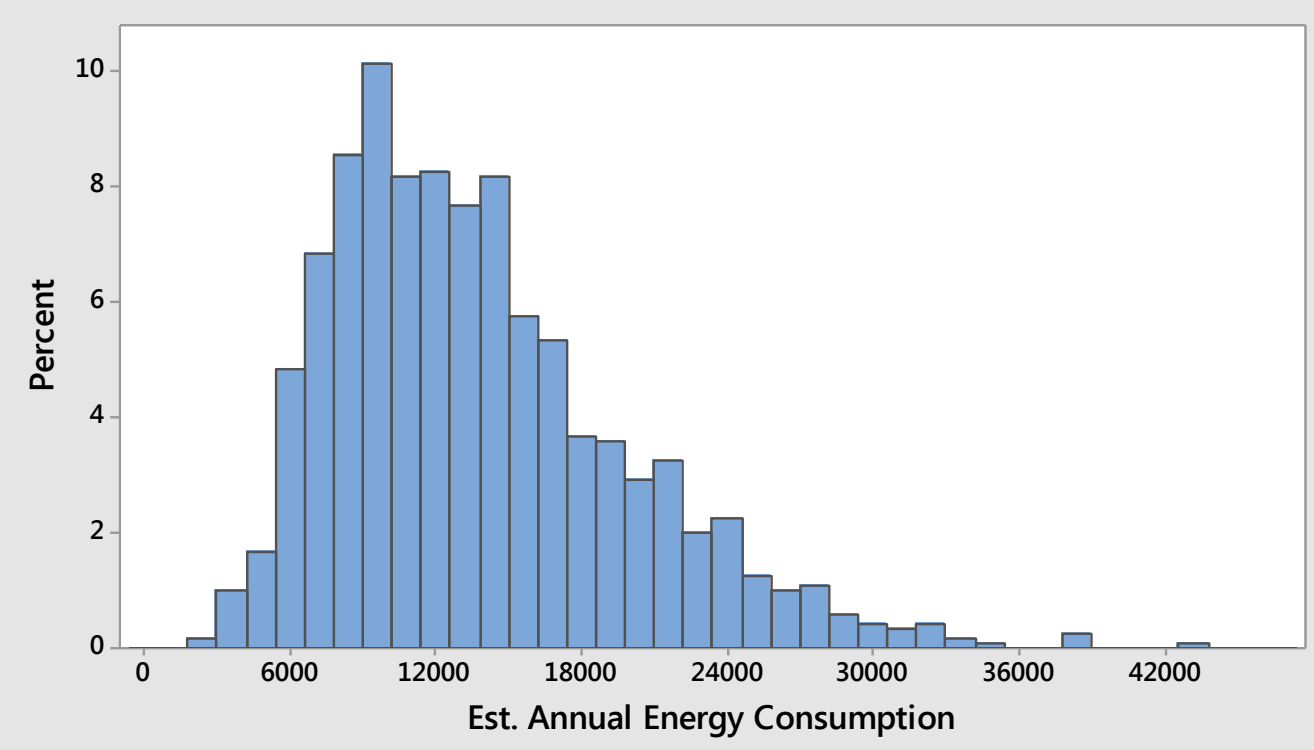

Figure 8: Histogram of annual energy consumption of 1200 homes in Glasgow, KY

It is possible to divide households into many groups based on annual income, but we chose only three groups to simplify our model development. Based on the census bureau data from 2010, we divided the population into three categories according to annual income. Table 1 shows the definition, criteria and distribution of these three categories. Low income households are those with annual income $\leq \$ 35000$, high income are those with annual income $\geq \$ 100000$, and the rest being classified as medium income.

Table 1: Classification of households according to annual income

\begin{tabular}{|c|c|c|c|}
\hline Group & Definition & Annual Income & Percentage \\
\hline A & Low & $\leq \$ 35 \mathrm{~K}$ & $36.6 \%$ \\
\hline B & Medium & $\$ 35 \mathrm{~K}-\$ 100 \mathrm{~K}$ & $43 \%$ \\
\hline C & High & $\geq \$ 100 \mathrm{~K}$ & $20.5 \%$ \\
\hline
\end{tabular}


The risk nature of a household can be an additional method of classification. It is not uncommon to consider different utility functions for people with different risk natures. The risk attitude of a consumer describes his or her marginal utility during decision making. Energy usage patterns can also be shown to have varying profiles indicating the behavior of the consumer. Load factor is a parameter that defines the fluctuation of a load profile for a specific time period. It is calculated by taking the average load divided by the peak load for the specified period. A high load factor indicates a relatively constant energy usage, while a low load factor shows that there are occasional high usage or load spikes. Different types of load factors can be indicative of a variable risk nature of consumers.
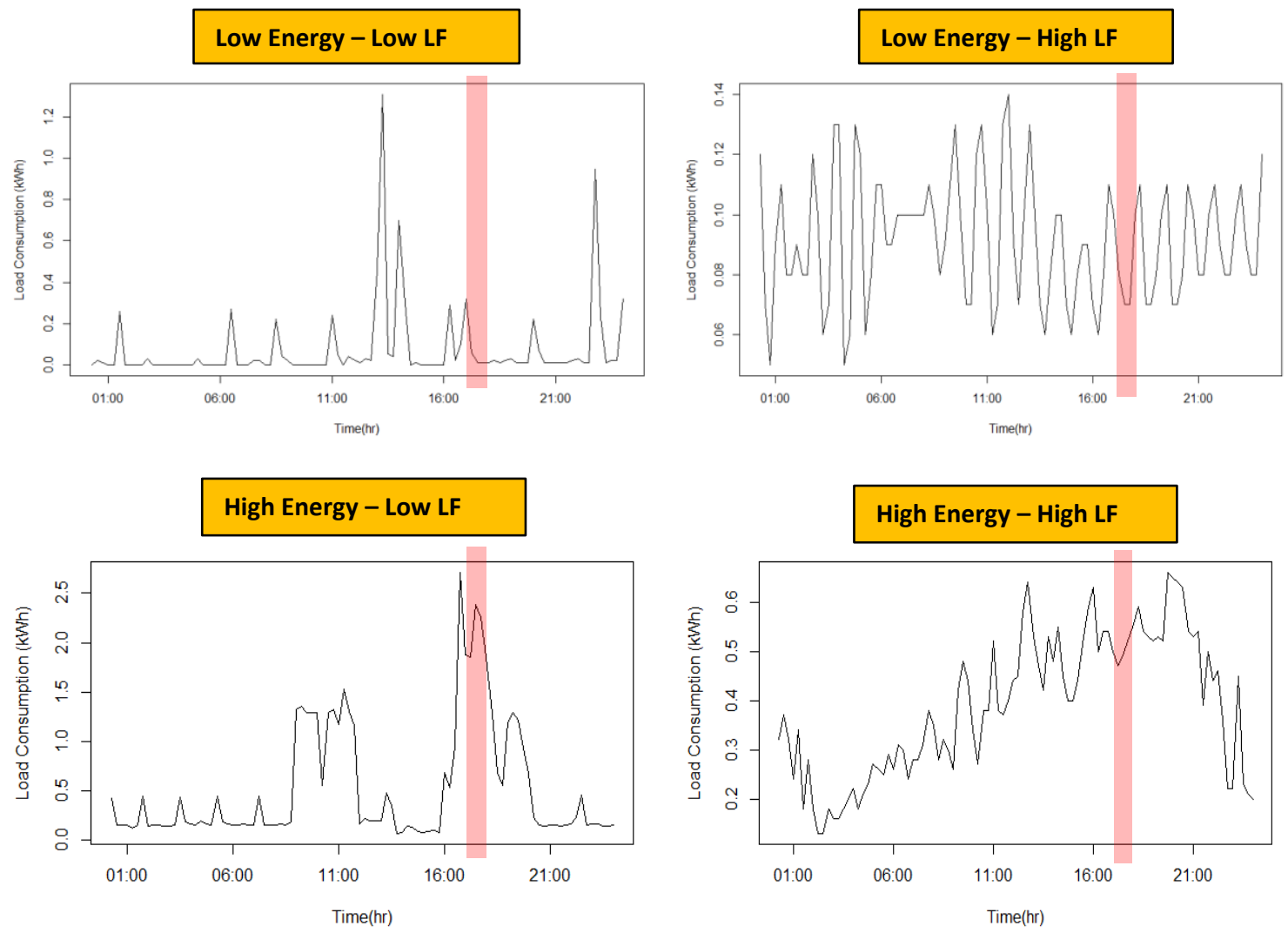

Figure 9: Load profiles of four different homes showing variable consumption patterns 
Figure 9 shows an example of different load profiles. From the data obtained from Glasgow Electric Plant Board (GEPB) of 1200 homes in 2014, the load factor of each home was calculated and averaged across 365 days. Homes were categorized based on their load factor and annual energy consumption. The figure shows the load profile of four homes on a particular date (Jan 6, 2014). These four homes were chosen to have different combinations of energy usage and average load factor. We can see a diverse load profile indicating the variation of load consumption pattern. Both homes with high load factor have a relatively consistent consumption, only differing in the amount of energy drawn. The homes with low load factor can be seen to have a spike of energy usage exceeding even the maximum load of its counterpart. The red band indicates the system peak for that day.

The above figure suggests that the average load factor (or behavior) of a house is independent of the total consumption. This means that the consumption behavior (consistent or spikey) does not depend on the amount of energy consumed, consequently the income group. When we see the distribution of the average load factor of the 1200 homes, we observe a normal distribution with a mean of 0.46 (Figure 10). When studying the distribution of load factor among various levels of annual energy consumption, we do not see any significant dependency (Figure 11). Because the risk nature of a household is also a rather subjective matter, we assume that each category is uniformly distributed with varying risk natures, which is discussed in detail in the next section. 


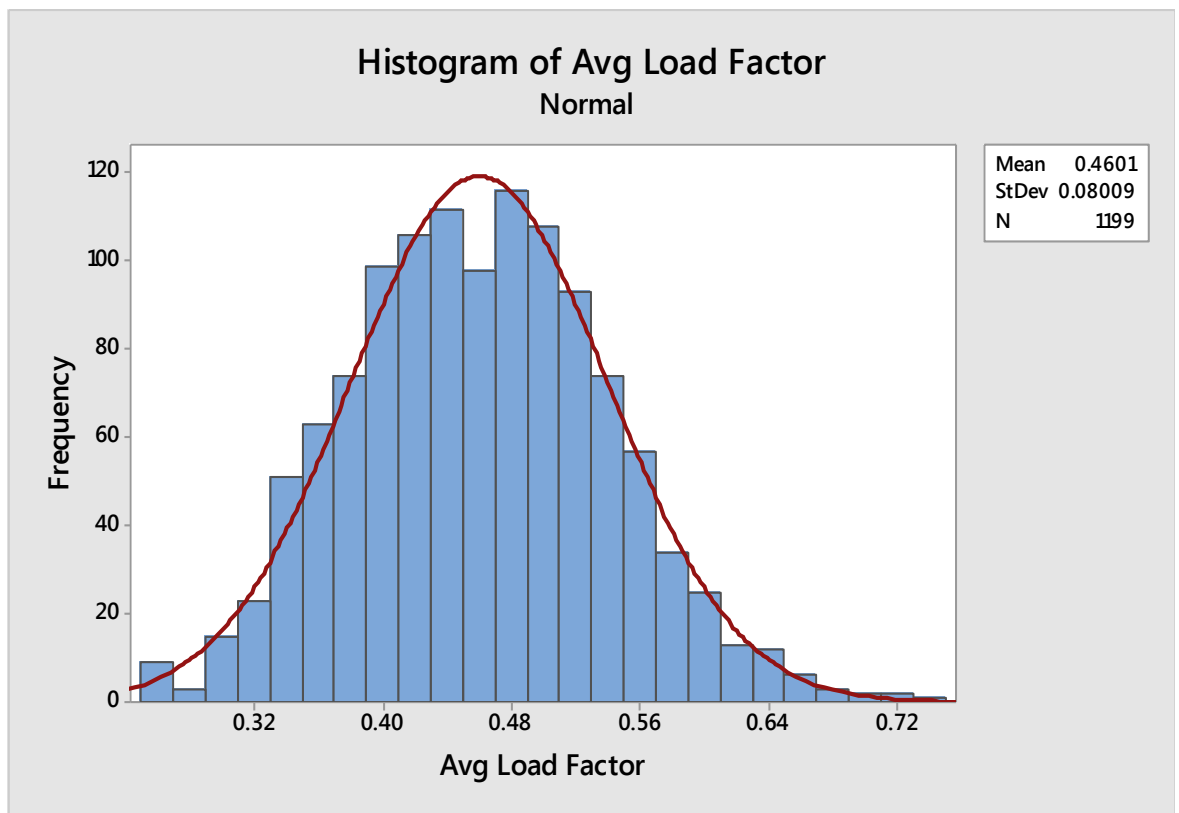

Figure 10: Normal distribution of average load factor for 1200 homes

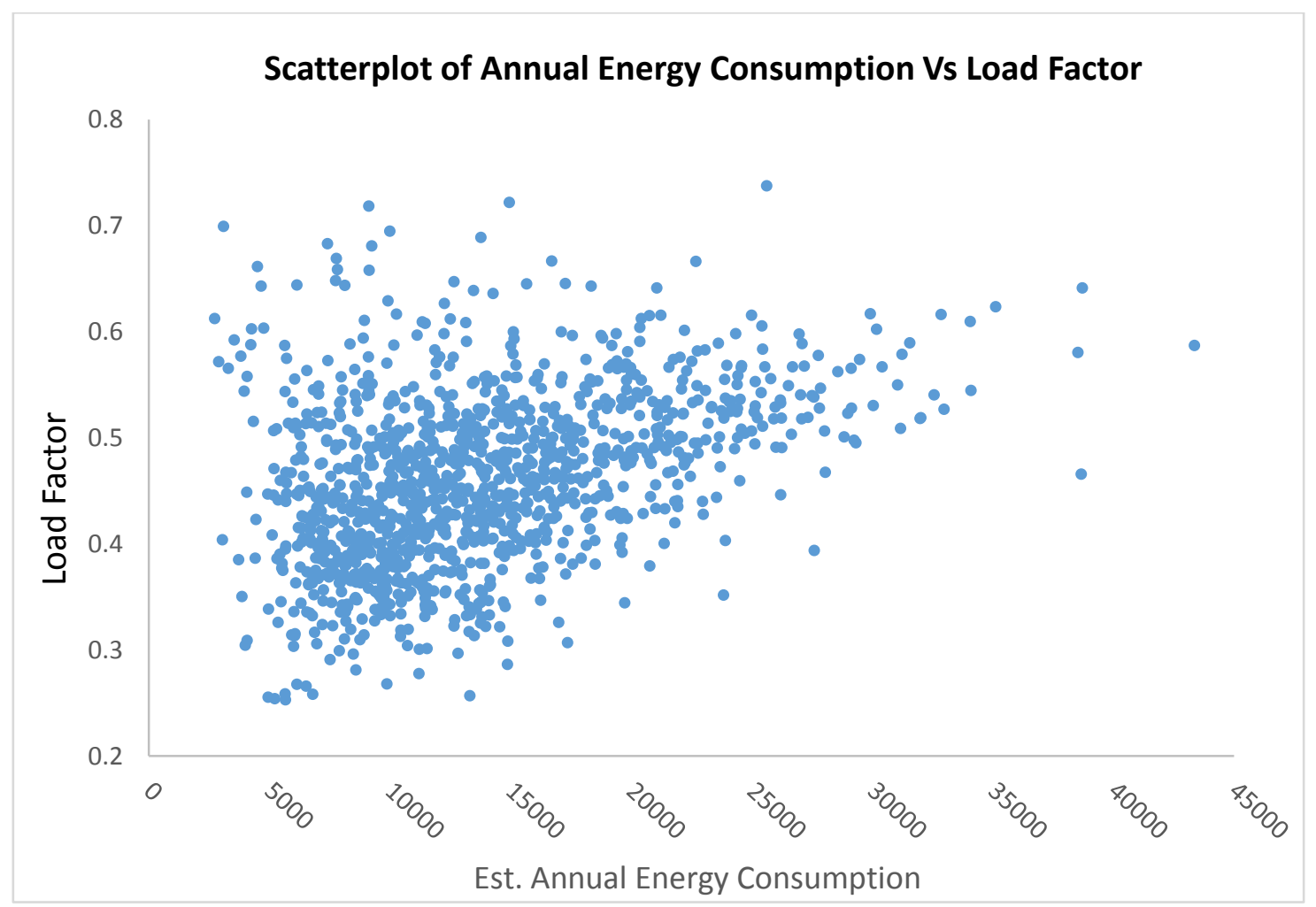

Figure 11: Annual energy consumption Vs Average Load Factor for 1200 homes 
For the purpose of estimating the load demand, we have also considered the number of occupants in a household as one classification criteria. Based on the census bureau data for 2010, households were divided into three size categories (Table 2). This classification is used for estimating the energy demand for non-thermostatic appliances for different households. When modeling thermostatic loads like HVAC, the energy demand is based on the thermal dynamics of the house and thus this classification will not be used.

Table 2: Classification based on number of occupants

\begin{tabular}{|c|c|c|}
\hline Definition & Number of occupants & Percentage \\
\hline Low & 1 & $35.2 \%$ \\
\hline Medium & 2 & $31.6 \%$ \\
\hline High & $\geq 3$ & $33.2 \%$ \\
\hline
\end{tabular}

\subsection{MULTI ATTRIBUTE UTILITY (MAU) FUNCTIONS}

As discussed earlier, the prevalent research and literature in demand side management (DSM) focus on financial incentives to motivate consumers to shift or reduce load usage. The concept of utility maximization has been pivotal in the modeling of consumer behavior and there are a lot of instances of its use in related energy economics literature. Nesbakken (1999) studied the price sensitivity in the choice of heating equipment and energy consumption. He defined an indirect utility function to represent the total utility derived by maximizing the consumer"s utility function. The indirect utility consisted of many terms defining various characteristics of the household such as type of house, household income, price of electricity, cost of choosing a heating 
system, etc. Fahrioglu and Alvarado (2000) use a benefit function to denote the value of a product and a cost function of electricity curtailment to design incentive based contracts. They use a normalized parameter in the benefit function to denote different characteristics of people based on how they value energy. Samadi et al. (2010) define the utility of energy usage as a quadratic function of the amount of energy used and a behavioral parameter. They represent the welfare of the consumer as the utility minus the cost of energy. In a distributed demand response model, Fan (2010) used a logarithmic utility function to define a consumer's willingness to pay a given cost. Pedrasa et al. (2010) proposed an energy scheduling optimization method using a decision-support tool. The scheduling algorithm consisted of the maximization of a fitness function, a linear equation of monetary benefit of energy equivalent and cost of consumption. Jian and Low (2011) used a game theory approach for optimal energy procurement using a user model and system model separately. They proposed a user utility for the user model, which measured the difference between a user"s target consumption versus actual consumption. $\mathrm{Xu}$ and Deng (2013) use an exponential utility function to model consumer choice between different contracts.

There are some papers (e.g., Avci et al., 2013 and Castilla et al., 2011) that include consumer convenience in their models, with variables such as discomfort index or thermal comfort using predictive mean vote (PMV). However, a majority of energy consumption modeling approaches using consumer utility focus primarily on the amount or cost of energy. Especially when studying the response behavior of a population to various DR programs, we feel it is important to consider the trade-off between cost and comfort. As we know that people behave in different ways, it must be taken into account 
that some people prefer comfort over cost savings. This is even more essential when analyzing the decentralized response of variable pricing, since people have diverse tradeoff behaviors, all of which affect the overall system performance.

We represent consumer behavior as being influenced by their perceived value of cost saving as well as convenience/comfort. We consider both to be major factors in the decision making process. In particular, the term "convenience" corresponds to NTL models, where the time of use for various appliances (clothes washer, dryer and dishwasher) defines the convenience factor. On the other hand, the term "comfort" corresponds to TCL models (HVAC) where the consumeres thermal comfort based on room temperature is considered. The development of the utility functions used in this dissertation is described in the following sections.

\subsubsection{Utility of Money and Exponential Functions}

In the study of economics and decision theory, utility is widely used as a measurement of satisfaction or welfare obtained from the procurement of any goods or service. It is usually applied to situations involving the demand and supply of various commodities, where the preferences of decision makers need to be taken into account. When faced with multiple options, say A, B and C, the preference of one over the other is denoted by the value of utility function $u($.$) of each option. If \mathrm{A}$ is preferred to B and B preferred to $\mathrm{C}$ (denoted by $A \gtrsim B \gtrsim C$ ), then the utility of each option must also follow $u(A) \geq u(B) \geq u(C)$. When faced with a choice of multiple variables, an indifference curve is generally used to plot the combinations of different variables that a person would accept to maintain a certain level of satisfaction. The indifference curve is 
simply a curve that passes through all points (variable combinations) with the exact same utility value. The utility function and indifference curve are separate tools in that the former allows us to find the best solution by maximizing total utility, whereas the latter allows trading off between different solutions with the same utility value.

Even though it is already in a numeric form, the use of utility to express the satisfaction gained from acquiring money has been prevalent in the study of economics. The value of money is said to have a non-linear form. The amount of happiness (utility) gained from getting a certain amount of money is greatly dependent on the initial amount possessed by the decision maker. Let"s say a person wins $\$ 500$ from a $\$ 1$ lottery ticket. The value of this winnings may be great for someone who currently only has $\$ 500$ in their bank account, meaning a doubling of their asset. For a richer person however, a $\$ 500$ winnings could be a mere $1 \%$ increase of their asset and the value might not be as great. It follows that the marginal benefit of gaining an extra dollar decreases as you have obtained more money. This means that the utility of money is a concave function, which partially explains the risk aversion of people. The higher the concavity, the larger is the risk aversion. It is to be noted that a risk-seeking attitude may also exist, which is defined by a convex function with increasing marginal utility. However this is rarely observed in the study of financial gain/loss, thus we ignore risk-seeking behavior.

As illustrated in Figure 12, the utility of money increases with the amount received. We can see that the utilities of various amounts of money, A $>$ B $>$ C, are denoted in the $y$-axis and maintain their corresponding preferential structure, $u(A)>u(B)$ $>\mathrm{u}(\mathrm{C})$. However, the marginal utility at $\mathrm{C}$ is much higher than at $\mathrm{B}$. For the same amount of increment, $(\mathrm{B}-\mathrm{C})=(\mathrm{A}-\mathrm{B})=x$, the change in utility is higher from $\mathrm{C}$ to $\mathrm{B}$ than from 
B to $A$, i.e. $[u(B)-u(C)]>[u(A)-u(B)]$. If a person has $C$ amount of money he/she values the increment of $x$ much more than if he/she has already obtained B. This property of a diminishing marginal utility will be discussed in the next section.

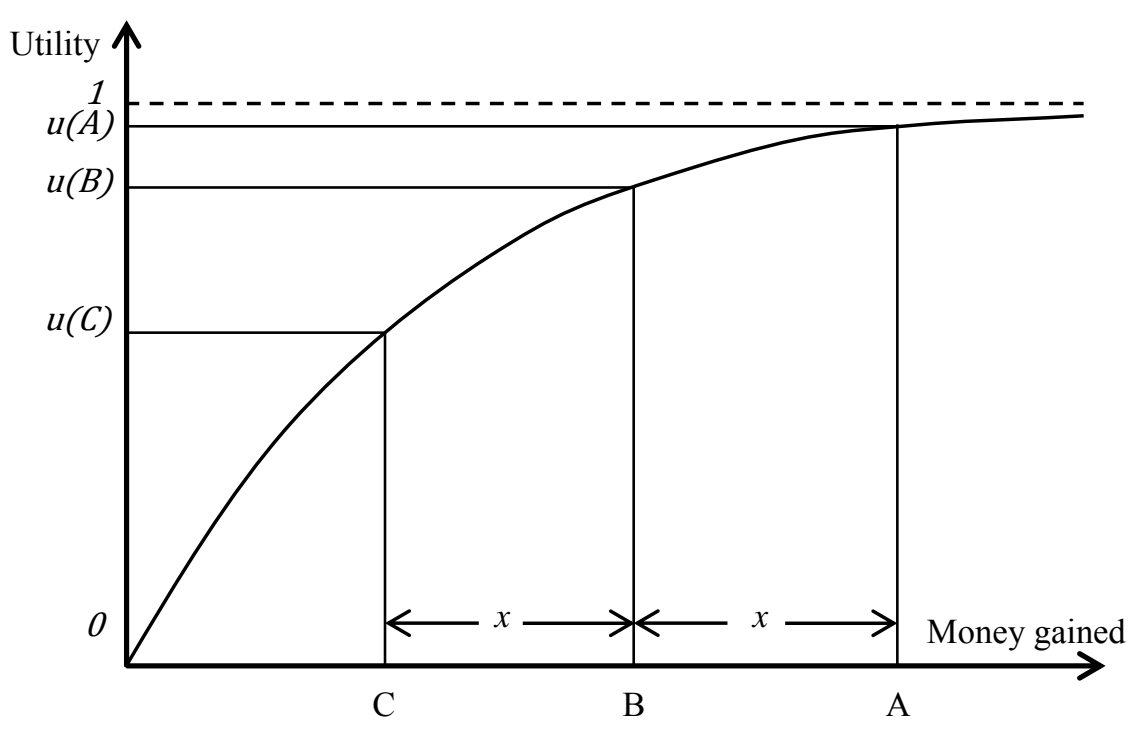

Figure 12: General form of the non-linear, increasing utility function of money

Various non-linear forms of utility have been used in the literature to represent the utility of money. Exponential functions are commonly used for this purpose, although quadratic and logarithmic forms have also been used in the literature (e.g., Samadi et al., 2010, Fahrioglu and Alvarado, 2000, Mohsenian-Rad et al., 2010, etc.). We propose the use of the exponential function since it nicely represents the bounded form of utility, which is measured between a scale of $[0,1]$. The standard exponential curve for defining utility functions involving risk is shown in Equation (1), where the constant $\mathrm{R}$ is known as risk tolerance. The risk tolerance affects the shape of the curve, thus making it more or less risk averse. The term "risk" in this context refers to the chance of incurring higher 
cost or experiencing higher discomfort. The classical method of representing economic utility with exponential functions takes advantage of the constant absolute risk aversion for concave functions.

When dealing with an attribute that can only take a finite and bounded set of values, we need to ensure that the curve is bounded not only by the utility values $[0,1]$ but also by the extreme values of the concerned variable $\left[X_{\min }, X_{\max }\right]$. Simply speaking, we expect to have two boundary conditions as $u\left(X_{\min }\right)=0$ and $u\left(X_{\max }\right)=1$. Thus, we use the general form exponential utility function given by Equation (2). The concavity of the curve is determined by $\alpha$ and the two boundary conditions are established and solved to obtain the final utility function.

$$
\begin{aligned}
& U(x)=1-e^{-\frac{x}{R}} \\
& U(x)=a+b e^{\alpha x}
\end{aligned}
$$

\subsubsection{Diminishing Marginal Utility}

The notion of a non-increasing marginal benefit from the utility of money is quite widely accepted. However, in our research we are interested in the value of cost rather than benefit. In the context of DR programs that incentivize consumers with rebates or other financial „,benefits ${ }^{e e}$, it is appropriate to use the above discussed forms of utility. But we are dealing with DR programs with variable pricings such that the intrinsic behavior of the consumer is affected by the cost of electricity. The behavior of consumers is subject to their attitude towards the loss of money instead of the benefit of receiving money. Thus we need to transpose the exponential functions given in Equations (1) and (2) such that we get a convex decreasing function with a non-decreasing marginal benefit. 
In behavioral economics, the nature of choice involving risk is also described by Kahneman and Tversky (1979) by using prospect theory. Although the decision making process is fundamentally different from utility theory, the value of money is described in a similar way. Prospect theory states that people make decisions based on the potential value of loss or gain rather than the final outcome. The utility function at any reference point is an asymmetric s-curve as shown in Figure 13. At the reference point, the slope of the function is greater for losses than for gains indicating a higher impact of loss. The asymmetric nature of the utility curve is not relevant when considering only one side of the graph. In modeling the utility of cost, we consider the left side of the curve starting at a value of one and decreasing to zero. If we are to consider cost in the positive domain, we will observe a reversal of the slopes. The marginal utility is highly negative when cost is at its minimum value but it increases as total cost becomes higher. This indicates a high dissatisfaction to lose the first dollar from a reference point, showing the general risk aversion of rational consumers when dealing with cost.

Our primary variables of interest are the cost of electricity and inconvenience or discomfort. From this point onwards, we will be using a decreasing convex utility function to denote the utility of cost as shown in Figure 14 . The utility of cost is maximum at the lower bound and minimum at the upper bound. The diminishing marginal utility holds in the absolute value, however we have a non-decreasing marginal utility when dealing with the utility of cost. The consumer prefers less of it contrary to a benefit function. 


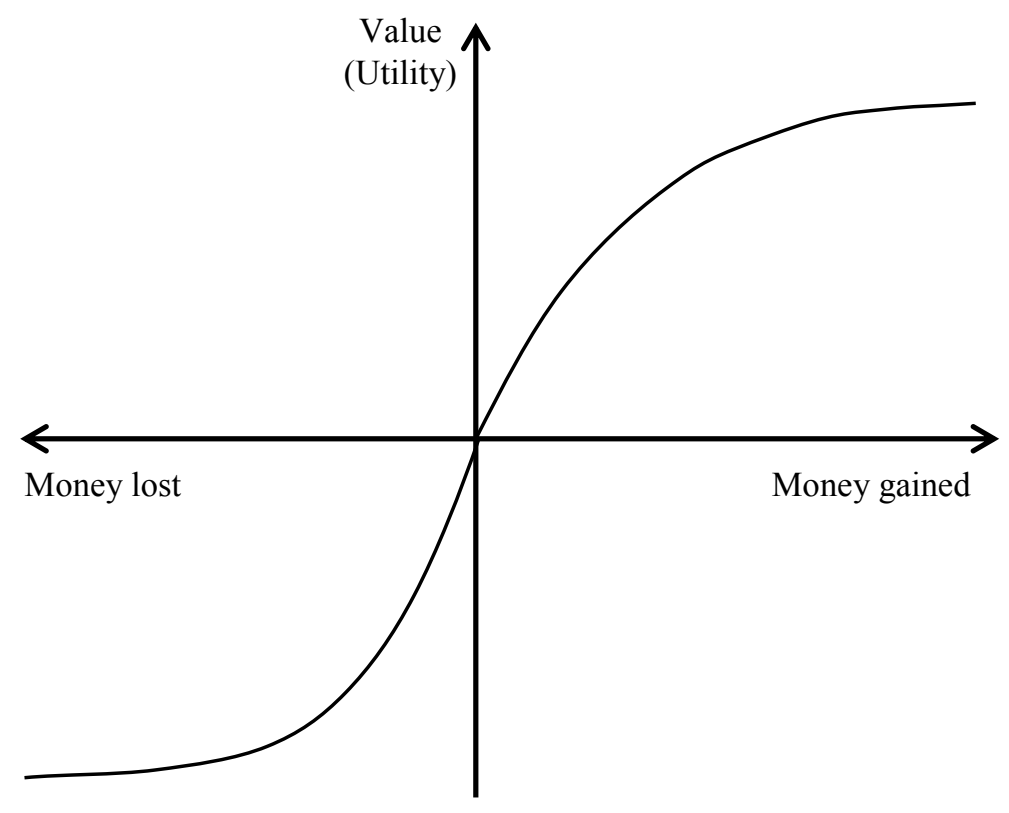

Figure 13: The value of money gained or lost, as viewed from a reference point

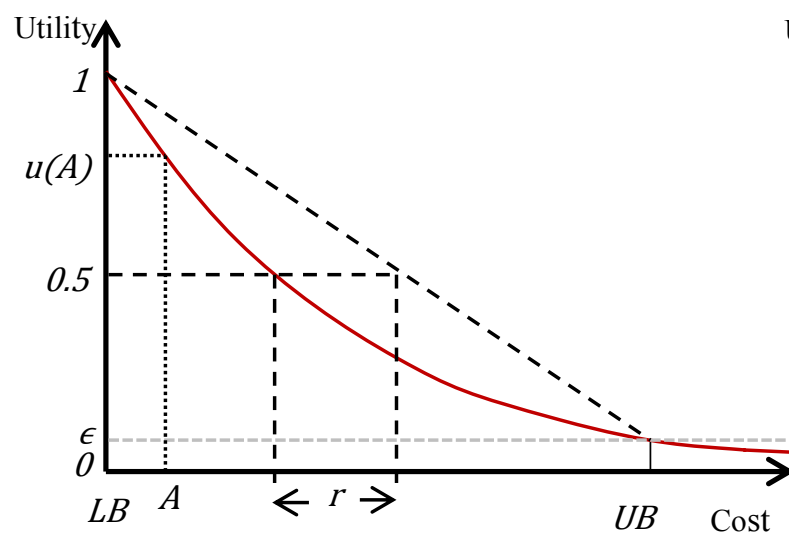

(a)

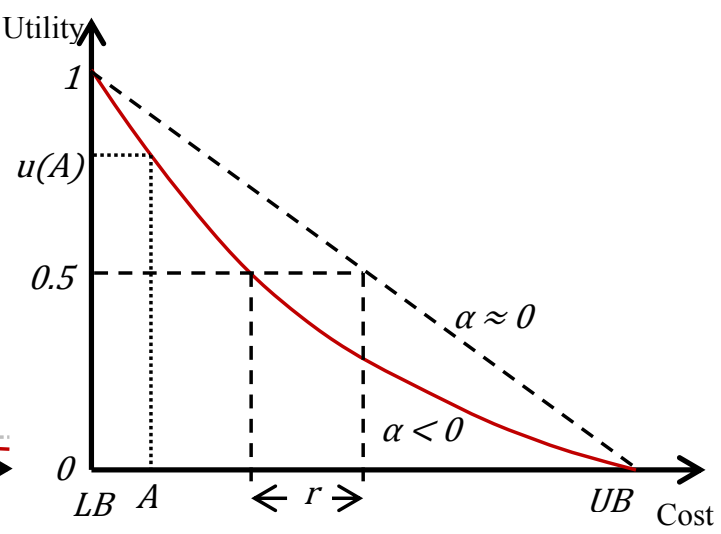

(b)

Figure 14: (a) Unbounded utility function of cost with arbitrarily small utility at upper bound, (b) Bounded utility function of cost with fixed upper bound at zero 
We use exponential functions to represent consumer utility. The standard exponential function given in Equation (1) is able to give a bounded value of utility between $[0,1]$ but it assumes that the value of the variable is unbounded. This means that the utility becomes zero at infinity. However, in practice the cost variables have a finite range of possible values, indicated by the lower bound $(L B)$ and upper bound $(U B)$ in Figure 14. The utilities evaluated at $L B$ and $U B$ should theoretically be one and zero, respectively. We can assign a pre-specified parameter $\epsilon$ with a very small value to denote the utility of UB, and modify the standard form given by Equation (1) to represent the pseudo-bounded utility function of cost. The modified exponential utility function for an unbounded variable is given in Equation (3), where $\alpha$ represents the degree of risk aversion based on the risk premium $r$ of the consumer (Figure 14a) and $\beta$ is carefully chosen to ensure $U(L B)=1$ and $U(U B)=\epsilon$. Risk premium basically denotes the minimum amount to the attribute that the consumer is willing to accept in compensation for the risk.

$$
\begin{aligned}
& U(x, \alpha)=\alpha e^{-\beta x} \quad(\alpha>0, \beta>0) \\
& \alpha=(\epsilon / 2)^{\left(\frac{r}{R}\right)-\left(\frac{L B}{R}\right)-\left(\frac{1}{2}\right)} \\
& \beta=\log \left(\frac{1}{\epsilon}\right) / R \\
& R=U B-L B
\end{aligned}
$$

Another form of exponential function can be used in order to ensure a bounded region for both the utility and the variable values. The decreasing convex function required to represent the non-decreasing marginal utility of cost can be obtained by 
modifying Equation (2) with the boundary conditions. The motivation for using a completely bounded form also stems from the concept of bounded rationality. In consumer economics, the theory of bounded rationality states that consumer rationality is dependent on the available information (Wilson and Dowlatabadi, 2007). Consumers do not spend a lot of time solving for the absolute optimal values, limited by the amount of cognitive processing and the time they are willing to spend calculating various options. It is stated from this theory that consumers make better decisions when they have a smaller number of options to choose from. Hence, we feel that a bounded utility function better defines consumer rationality. For example, if a consumer knows that the highest cost of electricity is $\$ 100$ even at the maximum energy consumption level, they can be sure that the utility is zero at that point. In contrast to this, if a consumer is faced with a potential cost that increases indefinitely, then the value of that cost becomes irrelevant after a certain point and their decision process becomes biased.

The bounded form of the exponential function that we use is given by Equation (7), where $\alpha$ denotes the risk aversion level of the consumer. As shown in Figure 14b, the risk aversion of the consumer is dependent on the risk premium $r$ and he/she becomes risk neutral when $\alpha \approx 0$.

$$
U(x, \alpha)=\left[\frac{e^{\alpha x}-e^{\alpha U B}}{e^{\alpha L B}-e^{\alpha U B}}\right]
$$

In order to maintain the diminishing marginal utility of a benefit function, we look to satisfy two conditions. First of all, a positive first derivative of the utility function indicates an increasing function. Then a negative second derivative indicates a decreasing marginal utility. Fahrioglu and Alvarado (2000) use a quadratic function $B(\theta, q)$ to 
represent the benefit of using $q$ units of energy by a consumer type $\theta$. This function is shown to have positive first derivative and negative second derivative, indicating a diminishing marginal utility. Samadi et al. (2010) also use a quadratic utility function $U(x, w)$ based on the energy consumption level $x$ and a parameter $w$ to define the type of user. They show that the function is increasing with respect to both $x$ and $w, U^{\prime}(x, w) \geq$ 0 and the marginal benefit is decreasing, $U^{\prime \prime}(x, w) \leq 0$.

The conditions are reversed for a convex decreasing function of cost. Yang et al. (2013) proposed the use of a dissatisfaction function based on the ratio of actual load consumption $l$ and nominal demand $d$. The consumer utility function $S(l, d)$ has the same form of a function, except that the dissatisfaction is measured in both positive and negative value. If the ratio $(l / d)$ is one, then the dissatisfaction function is zero indicating a neutral attitude. If this ratio is less than one the consumer experiences high dissatisfaction, and if it is greater than one the consumer has negative dissatisfaction (or satisfaction). Opposite to the benefit function discussed in previous paragraph, the dissatisfaction function used by Yang et al. shows a decreasing nature $\left(S^{\prime}(l, d)<0\right)$ and has a non-decreasing marginal dissatisfaction $\left(S^{\prime \prime}(l, d)>0\right)$.

We can see the first and second derivatives of our bounded exponential utility function in Equation (8) and (9). We can get $U^{\prime}(x, \alpha)<0$ and $U^{\prime \prime}(x, \alpha)>0$ only when the parameter $\alpha<0$. As mentioned earlier, we only consider risk averse nature although it is theoretically possible to have risk seeing behavior $(\alpha<0)$. We deem this as an unlikely behavior and thus only use $\alpha<0$.

$$
\frac{\partial U(x, \alpha)}{\partial x}=\frac{\alpha e^{\alpha x}}{\left(e^{\alpha L B}-e^{\alpha U B}\right)}
$$




$$
\frac{\partial^{2} U(x, \alpha)}{\partial x^{2}}=\frac{\alpha^{2} e^{\alpha x}}{\left(e^{\alpha L B}-e^{\alpha U B}\right)}
$$

\subsubsection{Definition of Cost Variables}

We use concepts from utility theory (Keeney and Raiffa, 1993; Clemen and Reilly, 2001) and develop multi-attribute utility functions to define the trade-off behavior of different households in response to DR. We first define the attributes of interest and develop the single attribute utility functions for each of them. Then we develop the multiattribute functions for each category of households indicating the different trade-off behaviors to cost and comfort. The central idea of this research is to model the response of consumers to the financial incentives of DR programs (variable pricing) in exchange for a potential inconvenience / discomfort. The model thus developed can then be used in designing optimal pricing structures. The two attributes that are central to our study are as follows.

- $\boldsymbol{X}=$ Cost of energy usage

- $\quad \boldsymbol{Y}=$ Cost of Inconvenience / Discomfort

Again, when considering non-thermostatic appliances, $Y$ is defined as the inconvenience caused by not being able to use an appliance at one $e^{\text {ee }}$ undesired time, scaled from 0 (most convenient) to 5 (most inconvenient). When considering thermostatic loads such as HVAC loads, $Y$ is defined as the discomfort experienced by the consumer measured as the deviation from a one ${ }^{e e}$ preferred temperature (thermal comfort).

In a smart grid system, it is assumed that relevant information such as price of electricity, forecast of prices, forecast of weather, projected savings, etc. is communicated 
with the customers via smart meters in real time. From the perspective of the consumer, there are two main factors that contribute to the change in energy usage. Firstly, the potential cost benefits or penalties incurred by using energy at different times of the day affects how much energy will be consumed at what times. Secondly, the comfort or discomfort experienced by such energy consumption behavior will also affect whether or not such pattern will be adopted. These two factors are conflicting in nature for DR programs mostly centered on incentivizing consumers for the reduction of peak loads. The cost of electricity is higher during peak hours and lower during off-peak hours. Thus the minimization of cost alone will naturally force most of the energy use during off-peak hours causing discomfort to the consumer. In contrast, if a person wishes to maximize his comfort by using energy during peak hours, it will incur a high cost. The nature of people in regards to their individual trade-offs between cost and convenience/comfort cannot be measured quantitatively. However, an approximation of the distribution of cost-favoring and comfort-favoring consumers can be made in a large population if the categories are reduced to a reasonable number as mentioned in Section 3.1.

The lower and upper bounds of the attributes represent the best and worst possible values desired by the consumer, respectively. The total energy drawn from the nonthermostatic appliances cannot be modeled easily. It is not possible to generalize the amount of time each appliance is operating. Thus we will use the rate of electricity as the pseudo cost variable instead of the actual dollar amount resulted by the use of the appliance. We assume that the preferred usage time is known and that this is flexible, thus the appliances are used whenever the rate is low. We assume that the standard residential electricity flat rate has a maximum value of $\$ 0.2 / \mathrm{kWh}$ and a minimum of 
$\$ 0 / \mathrm{kWh}$. We will use these as the extreme values for $X$ and hence set $U_{l}(X=0.2)=0$ and $U_{1}(X=0)=1$. The inconvenience of using an appliance at an undesired time is measured on a constructed scale of 0 to 5 , where 0 is the best possible value and 5 is the worst possible value. This is denoted as $U_{2}(Y=5)=0$ and $U_{2}(Y=0)=1$. The utility functions for $X$ and $Y$ are thus defined by Equations (10) and (11), by replacing the upper and lower bounds for each attribute in Equation (7).

$$
\begin{gathered}
U_{1}\left(X, \alpha_{1}\right)=\frac{e^{X \alpha_{1}}-e^{0.2 \alpha_{1}}}{1-e^{0.2 \alpha_{1}}} \\
U_{2}\left(Y, \alpha_{2}\right)=\frac{e^{Y \alpha_{2}}-e^{5 \alpha_{2}}}{1-e^{5 \alpha_{2}}}
\end{gathered}
$$

We estimate $\alpha_{1}$ and $\alpha_{2}$ based on risk premium, which determines the amount of an attribute that a person requires or is willing to give up, such that he will be indifferent to a chance outcome. The risk premium is the difference in the attribute value between the given function and a risk-neutral linear function at 0.5 utility, as shown in Figure 14. For a risk premium of $\$ 0.04 / \mathrm{kWh}$, the value of $\alpha_{1}$ is -9 . For the inconvenience cost, a risk premium of 1 hour delay gives the value of $\alpha_{2}$ equal to -0.4 . We can thus generate a population where $\alpha_{1}$ is uniformly distributed between $[-9,0)$ and $\alpha_{2}$ is uniformly distributed between $[-0.4,0)$ to simulate a varying degree of risk aversion.

The determination of cost variable is slightly more complicated when dealing with thermostatic loads. First of all the amount of time that an HVAC unit runs can easily be measured since it is dependent on the power rating of the unit, temperature set-point and external weather condition. Secondly, the flexibility of HVAC loads is not as high as other non-thermostatic appliances, since it doesn't make sense for a person to turn on the 
$\mathrm{AC}$ at night because the rate is cheaper and turn it off during midday just because it is more expensive. Thus only slight load shifts of a few hours may be achieved as a result. However, the base load of the house will interfere with this load shift as well. The base load is the total hourly load of a household minus the desired thermostatic load (e.g., HVAC). Load consumption data from 26 houses in Glasgow, KY that had sub-metering were studied to estimate the base load for various occupancy levels. Hourly load consumption data from 7/9/2012 to $8 / 26 / 2012$ were studied and a daily average was obtained, illustrative of a typical summer load profile. Since these homes had submetering from a previous smart appliance pilot study, we could particularly remove the HVAC loads and generate the required base loads for three groups with low, medium and high energy demand (see Figure 15). We can see a valley from 11:00 to 20:00, where most of the AC loads would have been.

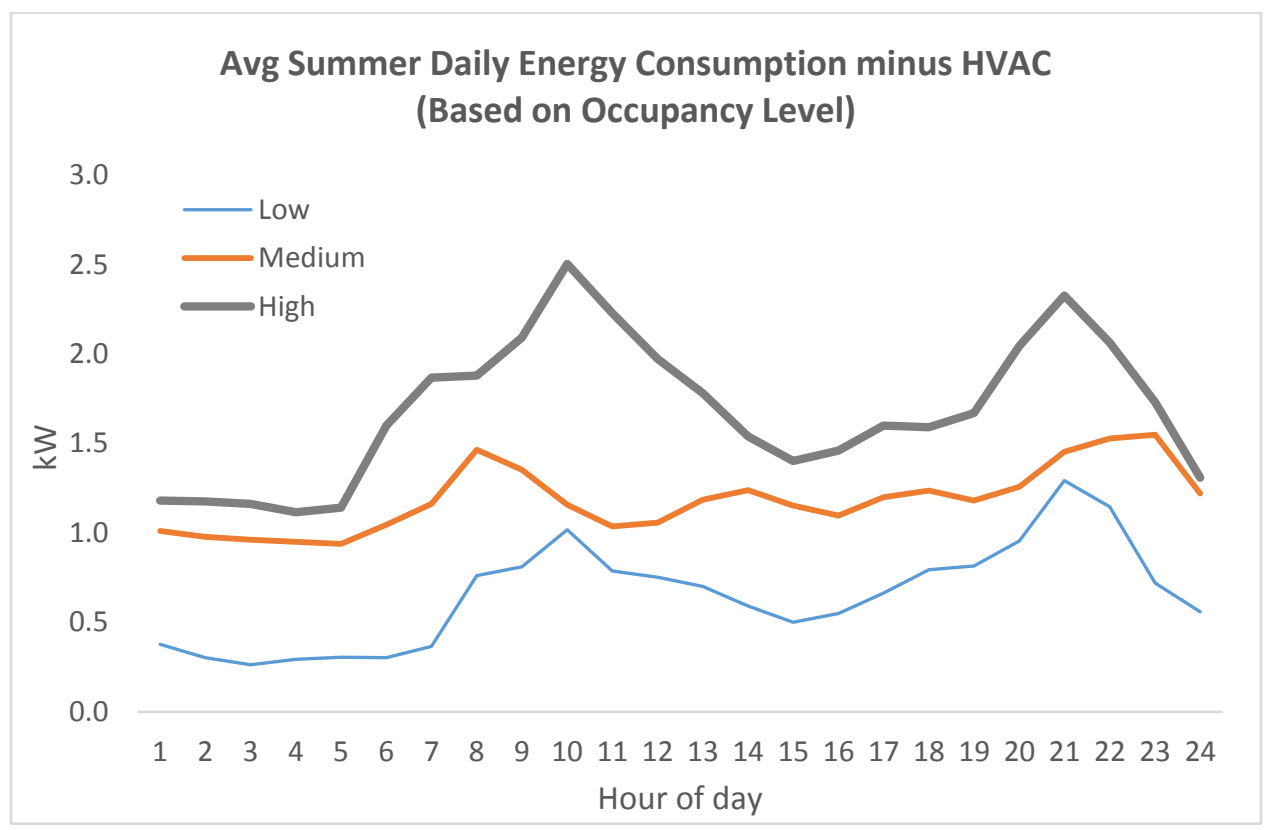

Figure 15: Estimated summer base load for various occupancy level homes 
The cost variable for this case will involve the actual dollar amount evaluated at every time interval. The decision interval is one hour for the purpose of this dissertation. We assume that the base load is fixed for any household and the consumer only decides whether or not to use AC at any given time. The base load has a minimum value $l_{\text {min }}$ and a maximum value $l_{\max }$. Given that the HVAC system of a house has a maximum rating of $q$, the maximum possible load is $\left(l_{\max }+q\right)$ and the minimum possible load is $l_{\text {min }}$. Thus the upper and lower bounds for cost are given by $p\left(l_{\max }+q\right)$ and $p\left(l_{\min }\right)$ respectively, where $p$ denotes the rate of electricity per $\mathrm{kWh}$ at a given time period.

The discomfort attribute in the context of thermostatic loads is defined as the deviation of room temperature from one ${ }^{\text {ee }}$ preferred value. The determination of the range of temperature is dependent on the preferred temperature for individual customer. For example, it is not uncommon for a person to feel most comfortable at $75^{\circ} \mathrm{F}$ while another person to feel the same at $65^{\circ} \mathrm{F}$. The estimation of this range of preferred temperature will be given in Section 4.2.1, where other thermal parameters are also identified. Any amount of deviation from this preferred temperature will cause a discomfort for the consumer and we consider a maximum deviation of $D_{t}$ to be least desired. Thus we have $U_{2}\left(Y=D_{t}\right)=0$ and $U_{2}(Y=0)=1$. The variation in internal room temperature is also observed to be different for different households. The estimation of $D_{t}$ will also be given in Section 4.2.1. We can obtain similar utility functions for cost and comfort by replacing the respective upper and lower bounds in Equation (7). 


\subsubsection{Measurement of Discomfort}

We initially define the total discomfort of a consumer as the amount of deviation of the room temperature $T$ from the preferred temperature $T_{p}$ of that consumer $(Y=$ $\left.\left|T-T_{p}\right|\right)$. We know that the utility is maximum when this value is zero and decreases as $Y$ gets larger. By this logic, under a flat rate pricing scheme where the utility of cost has minimum variation, the consumer would want to maximize their utility by keeping the room temperature $T$ as close to $T_{p}$ as possible, resulting in a consumption pattern shown in Figure 16. The preferred temperature $T_{p}$ of the simulated test house is set to $73^{\circ} \mathrm{F}$. However, this seems to be a naïve approach in defining thermal discomfort since actual inside temperature does not stay constant as shown in the figure. In observing the actual pattern of inside temperature of houses in the pilot study, we see a certain dependency on ambient weather. This leads us to believe that the preferred temperature of a consumer fluctuates with the external weather condition. In winter for example, a person may have a perceived comfort level at a much lower temperature than in summer. We call this the seasonal bias of thermal comfort.

When studying the actual average room temperatures of pilot homes, we observe that the ambient outside temperature has an effect on the inside temperature as well. The actual average inside temperature of the test houses under flat rate pricing schemes shows a pattern that follows the outside temperature $T_{o}$ to some extent. Figure 17 shows the actual readings of inside temperature for a test house with similar preferred temperature (Home 01: $T_{p}=73.24$ ). Immediately, we see that the average inside temperature for the 10 days is $66.54^{\circ} \mathrm{F}$, which is much lower than $T_{p}$. The average outside temperature for the same period is $37.88^{\circ} \mathrm{F}$, which seems to be reducing the comfort level of the house owner. 
We also see a distinct reduction in $T$ during night, when $T_{o}$ is low, and an increase during the day, when $T_{o}$ becomes higher, as can be seen in Figure 17. Since the inside temperature is allowed to fluctuate to a certain degree with the outside temperature, we also observe that the HVAC energy consumption is actually much lower than the simulated case (Figure 16) where the HVAC consumption would have had to be higher in order to maintain the inside temperature at $73^{\circ} \mathrm{F}$.

\section{Simulated temperature fluctuation from}

$1 / 1 / 2013$ to $1 / 10 / 2013$ for a test house
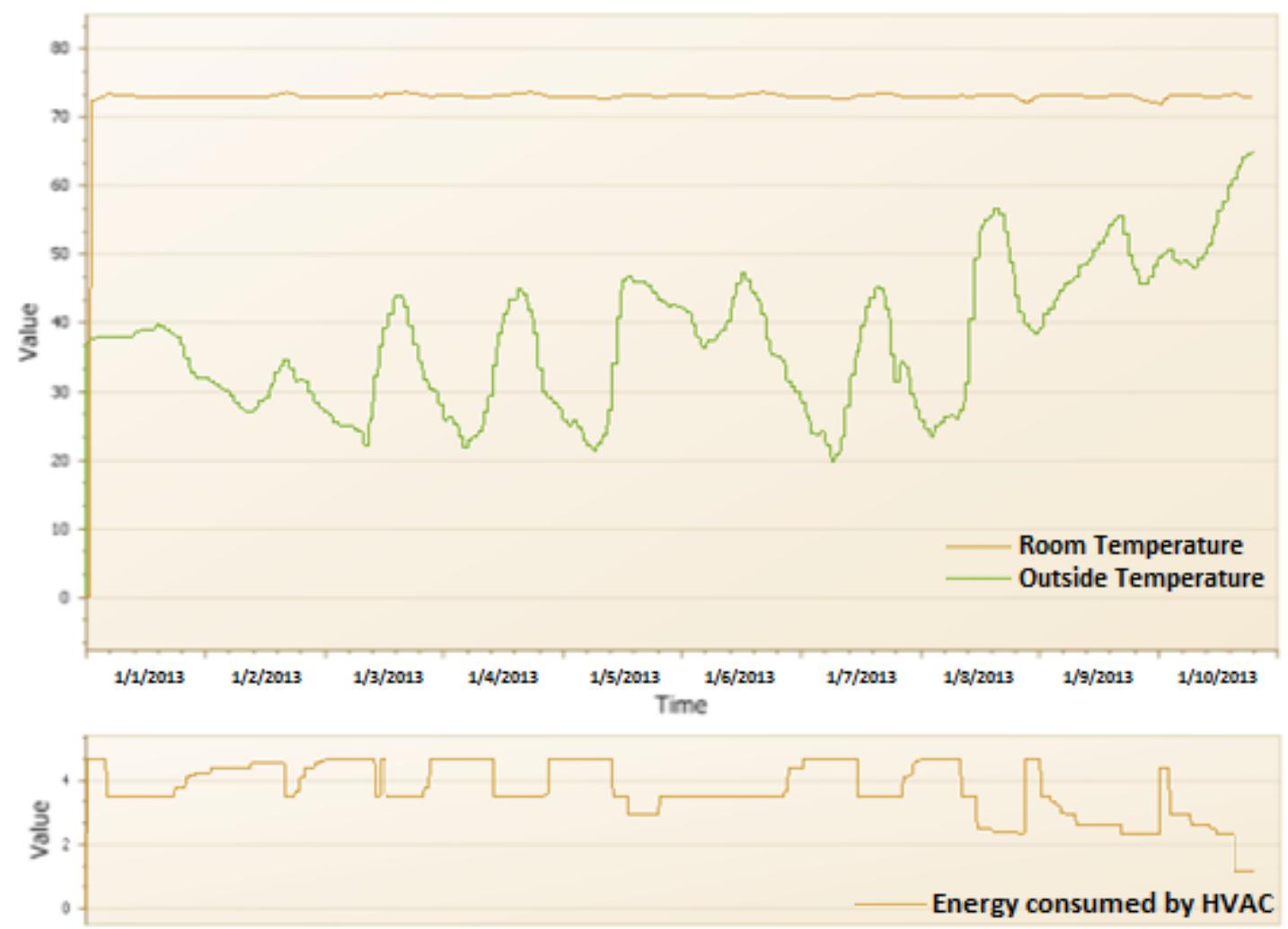

Figure 16: Simulated temperature profile of a test house for 10 days in January, 2013 


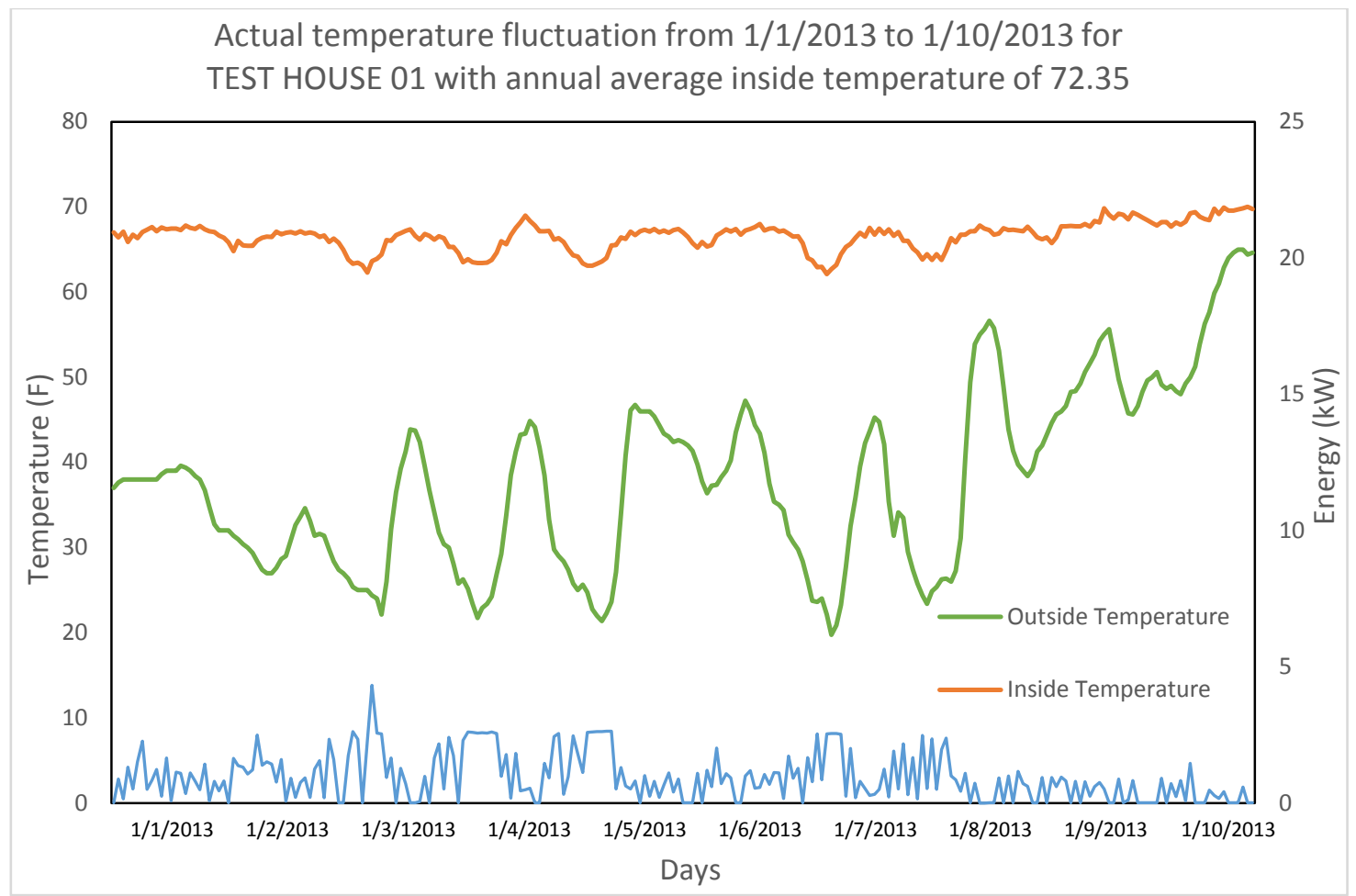

Figure 17: Actual temperature profile of HOME 01 for 10 days in January, 2013

This motivates us to propose the concept of actual versus absolute preferred temperature. The discomfort variable $Y$ will still be measured as the deviation from preferred temperature of the consumer, but this value will not be a constant. Rather the actual preferred temperature of a consumer will be the function of their absolute preferred value and the ambient outside temperature at any given time. The absolute preferred temperature is defined as the level at which a consumer feels most comfortable given that the outside temperature is also at the same level. Since the effect of outside temperature is the minimum, this will denote the perfect absolute preferred temperature for any consumer. We will develop this model empirically from the 2013 data of 10 test houses.

First of all we have to find the absolute preferred temperature. This is the temperature at which the consumer feels the most comfortable, given that the outside 
temperature is also same, thus eliminating seasonal bias. When we plot the average HVAC consumption per day against the average outside temperature for any house, we see a distinctive convex curve. The HVAC consumption is maximum at very low temperatures and it decreases with the increase in outside temperature, until a point where the HVAC consumption starts to increase again for higher temperatures (Figure 18). We cannot just look at data points where the inside and outside temperatures are the same and claim that it is the preferred temperature. This will soon lead to inconsistencies since inside and outside temperatures can remain at equilibrium regardless of the comfort of the consumer. In fact, if a consumer stops using HVAC altogether, the thermal equilibrium of outside and inside environment will always maintain.

The average daily inside temperature has a linear correlation with outside temperature. Figure 18 shows the standard relationship between HVAC consumption, average inside temperature and average outside temperature. In order to estimate the absolute preferred temperature, we find the optimal outside temperature $T_{o}{ }^{*}$ such that the HVAC consumption is at its minimum. Then the inside temperature at that point $T^{*}$ will be considered the absolute preferred temperature $T_{p}$ of a household. It is considered that the most comfortable temperature is at the point when HVAC consumption is at its minimum. We assume that the discomfort is always minimum when $T=T_{o}=T_{p}$. Figure 19 shows an example of the daily average HVAC load in $\mathrm{kW}$ and average daily inside temperature plotted against the average daily outside temperature for a test house, HOME 01. The optimal outside temperature for minimum $\mathrm{HVAC}$ load is equal to $64.55^{\circ} \mathrm{F}$, at which point the absolute preferred temperature $T_{p}=T^{*}=73.24^{\circ} \mathrm{F}$. 


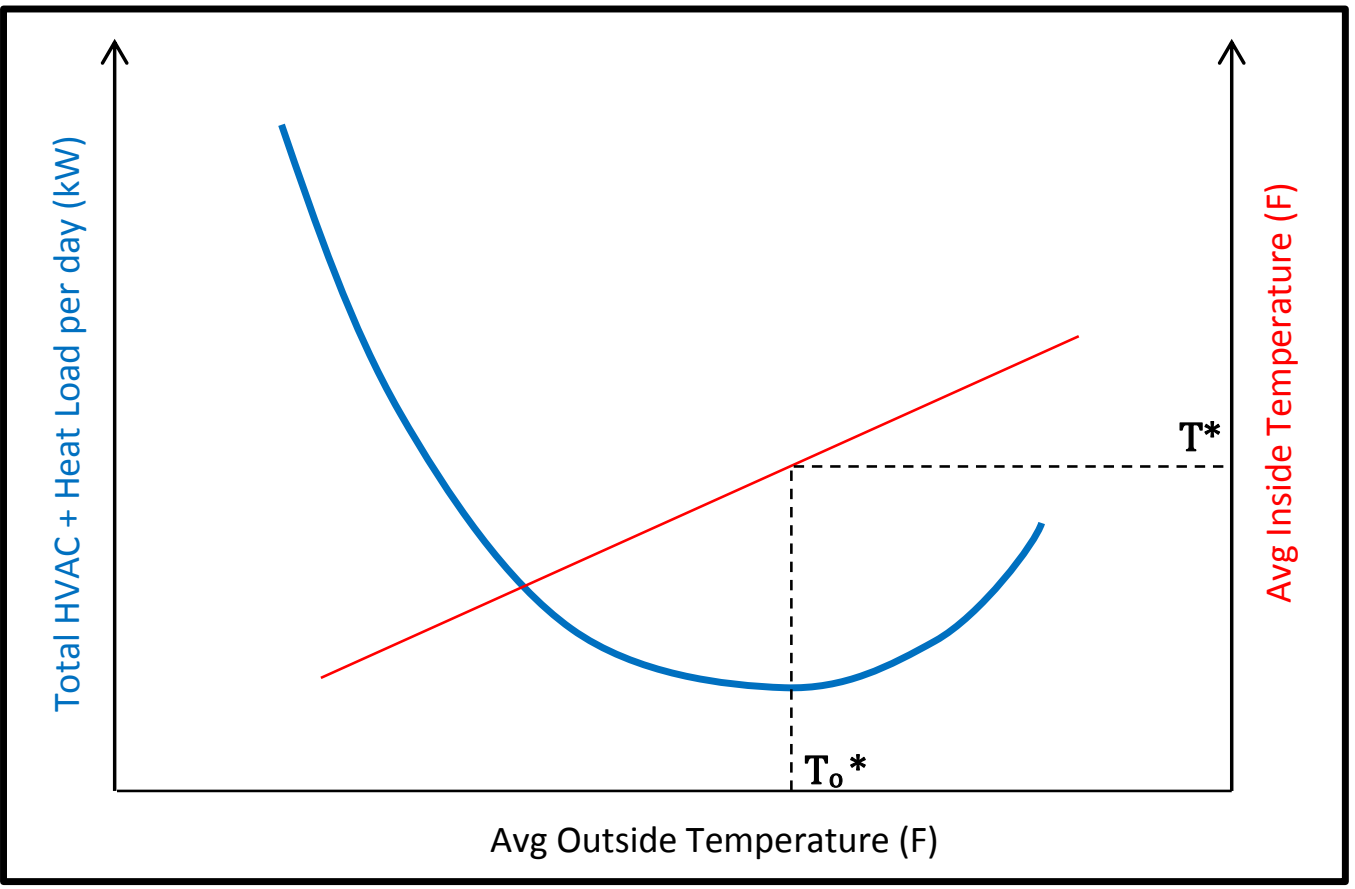

Figure 18: Plot of HVAC consumption and average inside temperature against average outside temperature to find absolute preferred temperature

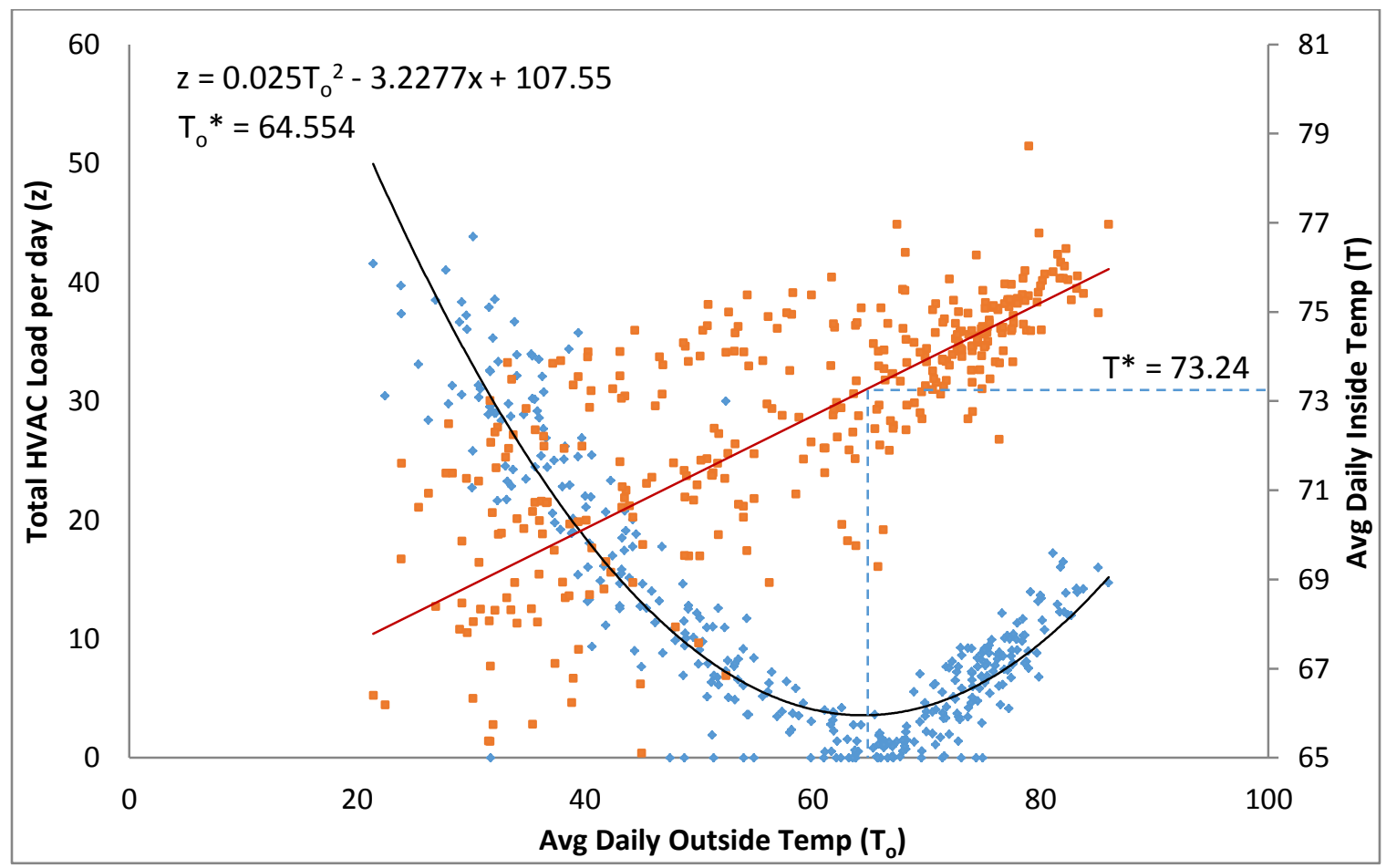

Figure 19: Example of the absolute preferred temperature estimation for HOME 01 
This brings us to the estimation of actual preferred temperature, which is affected by the ambient weather condition. The perceived comfort level for any person is experienced at a temperature lower than $T_{p}$ during winter and higher than $T_{p}$ during summer. We denote the actual preferred temperature by $T_{p}^{\prime}$, thus the actual measurement of discomfort being given by $Y=\left|T-T_{p}^{\prime}\right|$. Let us use a parameter $D_{t}=\left(T-T_{p}\right)$ to represent the total deviation of room temperature from absolute preferred temperature. This is not an absolute value and not similar to $Y$. We have already established that the effect of outside temperature $T_{o}$ partially accounts for this deviation along with any discomfort experienced by the consumer. When we plot $D_{t}$ against $T_{o}$ using hourly data for any of the 10 sub-metered test houses, we observe a linear relationship along with a Gaussian noise (Figure 20 shows an example plot for Home 01). The deviation $D_{t}$ from absolute preferred temperature can thus be attributed to a linear function of $T_{o}$ plus a random noise. We conjecture that this random noise explains the discomfort of the consumer and write Equation (12). The discomfort is denoted by $y$ which is $\leq 0$ during winter and $\geq 0$ during summer.

$$
\begin{aligned}
& D_{t}=\theta_{1}+\theta_{2} T_{o}+y \\
& T-T_{p}=\theta_{1}+\theta_{2}\left(T_{o}\right)+y \\
& y=\left\{T-T_{p}\right\}-\left\{\theta_{1}+\theta_{2} T_{o}\right\}
\end{aligned}
$$


Then by substitution we get Equation (14), which represents the discomfort value as the difference between $D_{t}$ and $D_{t}^{y=0}=\left\{D_{t} \mid y=0\right\}$. From the linear regression shown in Figure 20, we can see that $D_{t}^{y=0}$ is nothing but the fitted line when ignoring the random noise. Thus we can use the data for all 10 test houses to fit the equation $\left(D_{t}^{y=0}=\right.$ $\left.\theta_{1}+\theta_{2} T_{o}\right)$ and estimate distributions for $\theta_{1}$ and $\theta_{2}$. Uniform distributions were fit for both coefficients, $\theta_{1} \sim \operatorname{Uniform}(-18,-3)$ and $\theta_{2} \sim \operatorname{Uniform}(0.05,0.3)$. From the estimated values of $T_{p}, \theta_{1}$ and $\theta_{2}$ of the 10 homes, we find a linear correlation between $\theta_{1}$ and $\left(T_{p} * \theta_{2}\right)$. As shown in Figure 21 , a strong correlation $\left(\mathrm{R}^{2}=98.6 \%\right)$ between these parameters allows us to substitute $\theta_{1}$ in Equation (14). Since our sample size is only 10 , we make a slight assumption here by approximating the slope to -1 and the intercept to 0 . This leaves us with $\theta_{1}=-T_{p} * \theta_{2}$. Substituting this value we get Equation (15).

$$
\begin{aligned}
& y=T-\left\{T_{p}\left(1-\theta_{2}\right)+T_{o} \theta_{2}\right\} \\
& T_{p}^{\prime}=\theta T_{o}+(1-\theta) T_{p}
\end{aligned}
$$




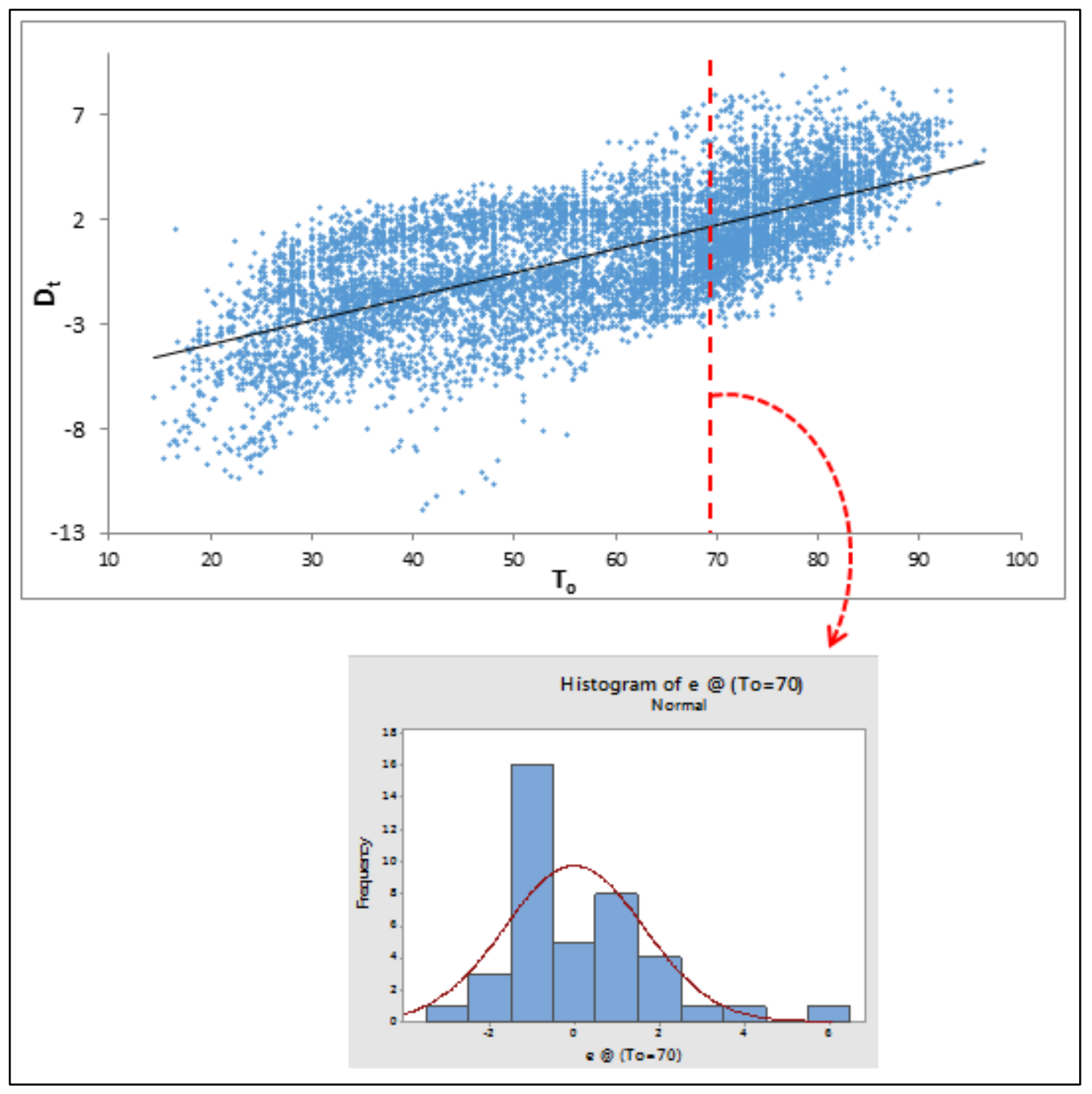

Figure 20: Example plot of deviation $\left(D_{t}\right)$ vs outside temperature $\left(T_{o}\right)$ for HOME 01

Since we claimed earlier that the discomfort must be measured as the deviation from actual preferred temperature rather than the absolute one, it follows that Equation (15) is in the form of $y=T-T_{p}^{\prime}$. Thus we can say that the actual preferred temperature is simply a convex combination of absolute preferred temperature and the outside temperature (Equation 16). We replace the coefficient $\theta_{2}$ by $\theta$ in order to simplify the notation since we are left with just one coefficient. 


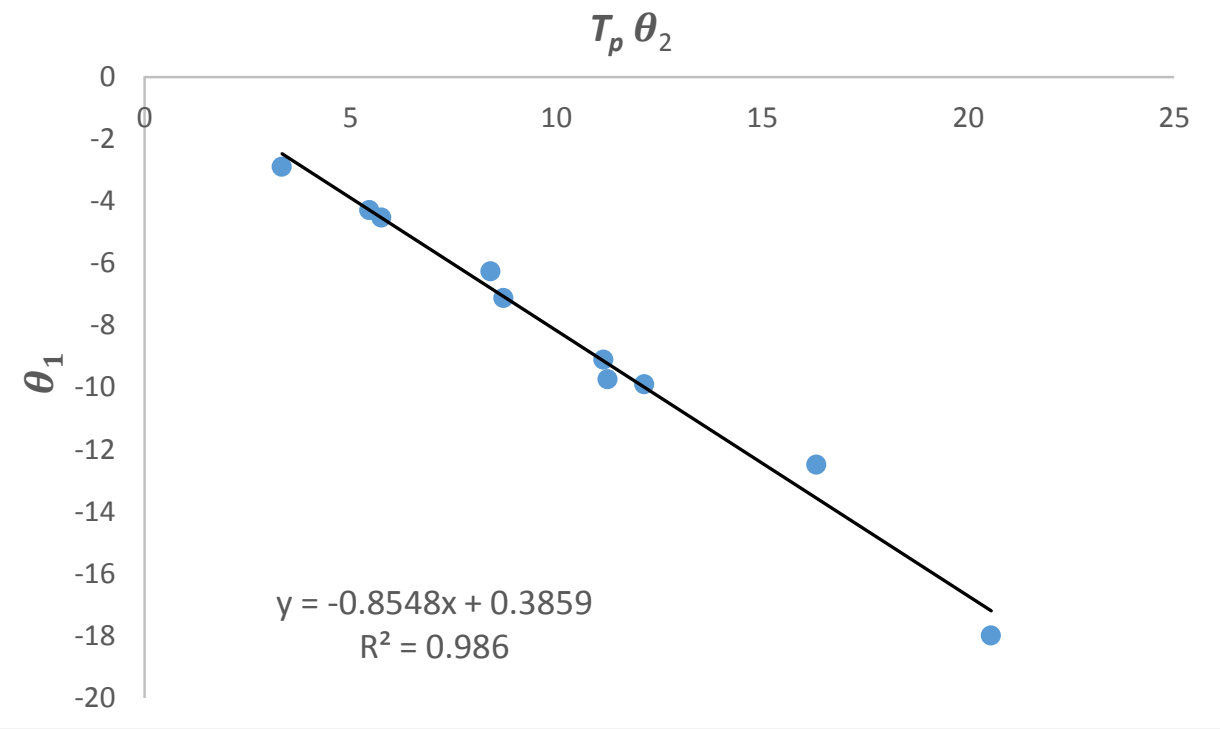

Figure 21: Linear correlation between $\boldsymbol{\theta}_{\mathbf{1}}$ and $\left(\boldsymbol{T}_{\boldsymbol{p}} * \boldsymbol{\theta}_{\mathbf{2}}\right)$

We know the distribution of $\theta$ to be uniform between $[0.05,0.3]$ from earlier. $T_{p}$ was replaced by $T_{p}^{\prime}$ in the simulation along with various values of $\theta$ to test the temperature profiles of a test house similar to Figure 16. Since $\theta$ is the effect of outside temperature on the actual preferred temperature, we see that as this value is increased the profile of inside temperature follows the outside temperature (Figure 22). We can see that different homes have different levels of $\theta$, meaning the effect of outside temperature on the actual preferred temperature of consumers are different. Those with low $\theta$ values will have an actual preferred temperature very close to their absolute value, thus trying to maintain a low variance in inside temperature. This reflects the nature of the household. Those with larger $\theta$ values have an actual preferred temperature strongly affected by ambient weather thus resulting in a higher variance in inside temperature. We finally conclude that discomfort is measured by $Y=|y|=\left|T-T_{p}^{\prime}\right|$. 

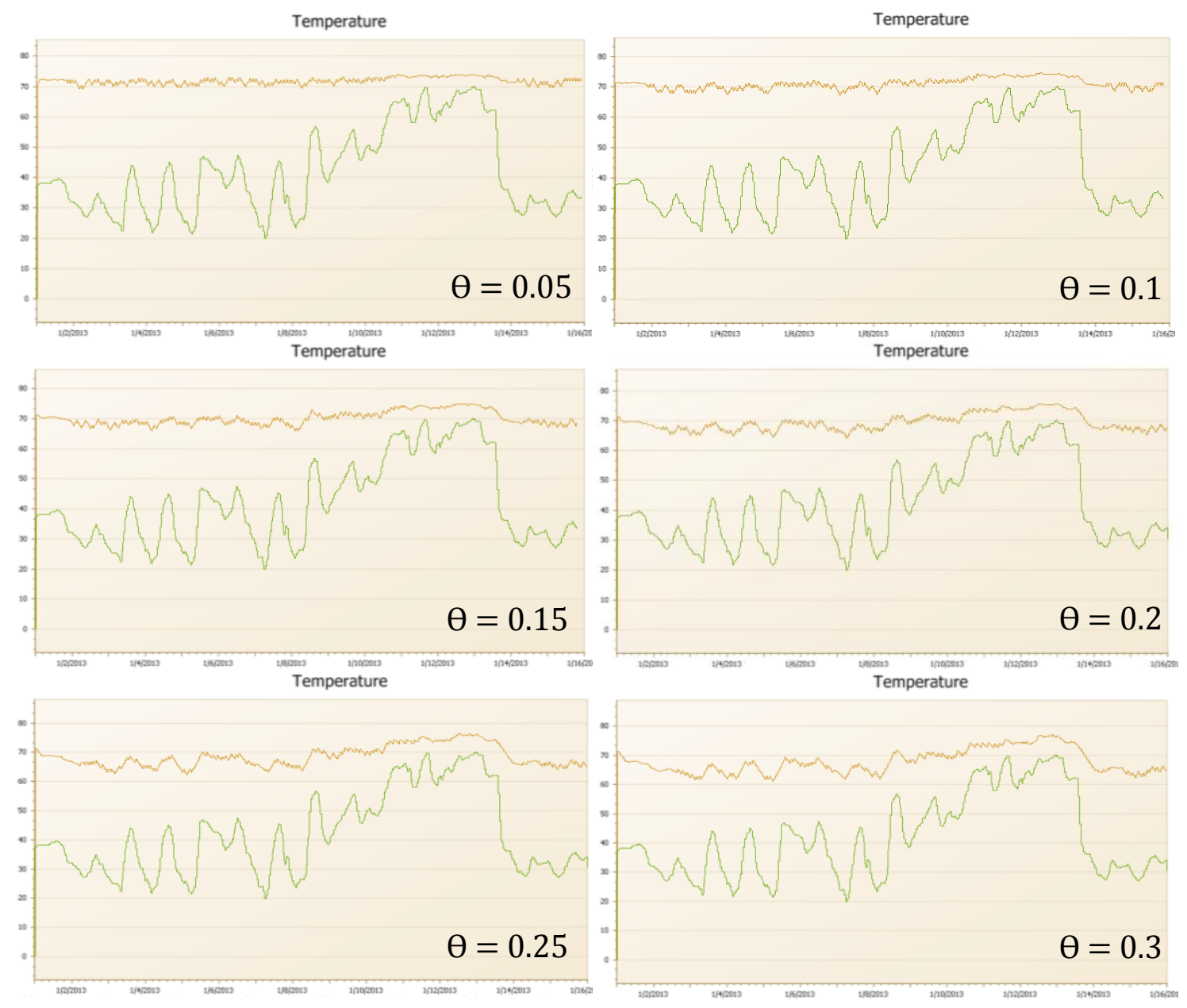

Figure 22: Simulation of room temperature (orange) based on outside temperature (green) for 10 days in January, 2013 at different levels of $\boldsymbol{\theta}$

\subsubsection{Utility of Energy and Demand Charge}

The utility function of cost for both non-thermostatic and thermostatic loads models have been defined earlier in this chapter. We have simplified the non-thermostatic case by using Equation (10), under the assumption that usage times are flexible for appliances such as washer, dryer and dishwasher, hence only considering the rate of electricity $p$ as a driving factor for utility. We proposed a slightly different method for thermostatic case since the loads are not as flexible. The utility of $X$ is based on actual 
dollar value from energy usage, hence replacing $L B=p\left(l_{\min }\right)$ and $U B=p\left(l_{\max }+q\right)$ in Equation (7). However, this is a general form of the utility function for cost assuming that energy cost is evaluated by a single variable rate $p$. In most pricing structures such as TOU, CPP, RTP, etc. this works fine since the energy drawn during a particular hour is charged a certain rate. For pricing mechanisms involving demand charge, the cost of electricity is evaluated as a sum of energy charge and demand charge. Depending on the nature of demand charge used by the utility company, the peak load for which demand charge is applied is billed twice: the energy charge plus the demand charge. To account for this we need to adjust the use of the utility function in such case.

If the values of energy and demand charge were to be somehow comparable in the same range, we could use a single utility function for cost. The rate of electricity $p$ could simply be the sum of energy and demand charge rates for the hour in which peak load were to be evaluated. However, the nature of demand charge is not similar to energy charge. Demand charge is currently being used in various ways as discussed in Section 2.4. Before it was introduced in DR programs for residential consumers, industrial consumers have long been subject to demand charge in order to discourage high power consumption for short intervals. For the same amount of total $\mathrm{kWh}$ energy consumption, the maximum power demand can be different if the time interval is not the same. $5 \mathrm{~kW}$ power used for 10 hours amounts to the same $\mathrm{kWh}$ as $50 \mathrm{~kW}$ used for 1 hour. But the high power usage for short time causes unstable demand and thus is detrimental to the grid stability. For this reason, instead of charging just the energy usage, a demand charge for the peak power is evaluated with a high value. Usually demand charge, assigned to the peak load $\mathrm{kW}$, is much higher in comparison to the standard energy rate. Demand 
charge could potentially account for anywhere between $30 \%$ and $70 \%$ of the total bill for industrial customers (www.stem.com). Some estimates have put this share at up to $30 \%$ for residential customers. For example Duke Energy in Charlotte, NC has an optional energy contract for its residential consumers that applies TOU with demand charge. In this rate structure the average energy charge is $6.32 \not / \mathrm{kWh}$ while the demand charge is $\$ 7.77 / \mathrm{kW}$.

Since energy and demand charge have vastly different ranges, we treat the total cost as the sum of the two and use different utility functions for each. Let $p_{e}$ and $p_{d}$ be the rates for energy and demand charge, and $X_{e}$ and $X_{d}$ denote the cost incurred due to either charge for a given time interval. We denote the total cost of electricity as the sum of the two for any given time $\left(X=X_{e}+X_{d}\right)$. Only the hour in which the peak load occurs will have the demand charge part, but this is not known in advance to the consumer and hence we assign $X_{d}$ with a probability. This will be discussed in detail in Section 4.4. Let the lower and upper bounds for $X_{e}$ be $\left[\ell_{e}, \mathcal{L}_{e}\right]$ and the same for $X_{d}$ be $\left[\ell_{d}, \mathcal{L}_{d}\right]$. From the earlier discussion of upper and lower limits of cost for TCL models in Section 3.2.3, we have the following where $l_{\min }$ and $l_{\max }$ are the limits of base load and $q$ is the HVAC power rating.

$$
\begin{aligned}
& U_{e}\left(\ell_{e}\right)=1, \quad \text { where } \ell_{e}=p_{e}\left(l_{\text {min }}\right) \\
& U_{e}\left(\mathcal{L}_{e}\right)=0, \quad \text { where } \mathcal{L}_{e}=p_{e}\left(l_{\text {max }}+q\right) \\
& U_{d}\left(\ell_{d}\right)=1, \quad \text { where } \ell_{d}=p_{d}\left(l_{\text {min }}\right) \\
& U_{d}\left(\mathcal{L}_{d}\right)=0, \quad \text { where } \mathcal{L}_{d}=p_{d}\left(l_{\text {max }}+q\right)
\end{aligned}
$$

Let the utility of total $\operatorname{cost} X$ be represented by some combination of individual utilities of $X_{e}$ and $X_{d}$, as shown in Equation (17). It is to be noted that we are considering a simple 
additive form of the mixed utility. Since both $X_{e}$ and $X_{d}$ are cost variables in the same units it is easy to show additivity. A utility function is additive if and only if, for the two indifferent trade-offs such as $\left\{x_{1}, y_{1}\right\} \sim\left\{x_{2}, y_{2}\right\} \sim\left\{x_{3}, y_{3}\right\}$, Equation (18) is satisfied (Delquie and Luo, 1997). Basically the sufficiency condition is that given three points that are preferentially equivalent, the two slopes in utility space are equal. We can take an example with three random points, such as $\{\$ 0, \$ 10\} \sim\{\$ 2, \$ 8\} \sim\{\$ 4, \$ 6\}$. These three points are preferentially equivalent since we defined total cost as the sum of $X_{e}$ and $X_{d}$. We can clearly see that Equation (18) is satisfied, thus allowing us to use the additive form of total utility shown in Equation (17). When dealing with attributes that are not directly comparable in value, showing additivity is not as simple. This is discussed in the next Section 3.2.4.

$$
\begin{gathered}
U_{1}(X)=\beta_{e} * U_{e}\left(X_{e}\right)+\beta_{d} * U_{d}\left(X_{d}\right) \\
\frac{\left(y_{1}-y_{2}\right)}{\left(x_{1}-x_{2}\right)}=\frac{\left(y_{1}-y_{3}\right)}{\left(x_{1}-x_{3}\right)}
\end{gathered}
$$

We know that the total cost is minimum when both energy and demand charge are minimum and maximum when both are maximum. Thus we can write the upper and lower bounds of $X$ as $X_{\min }=\ell_{e}+\ell_{d}$ and $X_{\max }=\mathcal{L}_{e}+\mathcal{L}_{d}$. From the limits of the utility function, we have $U_{1}\left(\ell_{e}+\ell_{d}\right)=1$ and $U_{1}\left(\mathcal{L}_{e}+\mathcal{L}_{d}\right)=0$. Thus, when both $X_{e}$ and $X_{d}$ are at their respective minimum values, Equation (17) reduces to Equation (19). We can see that the sum of the coefficients is one (Equation 20), thus the utility of total cost $U_{1}(X)$ must be a convex combination of individual utilities $U_{e}\left(X_{e}\right)$ and $U_{d}\left(X_{d}\right)$. Equation (20) is also a result of the additive form. 


$$
\begin{gathered}
U_{1}\left(X_{\text {min }}=\ell_{e}+\ell_{d}\right)=\beta_{e} * U_{e}\left(X_{e}=\ell_{e}\right)+\beta_{d} * U_{d}\left(X_{d}=\ell_{d}\right) \\
\beta_{e}+\beta_{d}=1
\end{gathered}
$$

The values of $\beta_{e}$ and $\beta_{d}$ are selected such that the preferential structure of energy and demand charge is maintained. Similar to above, we get Equations (21) and (22) by replacing combinations of minimum and maximum value for each of $X_{e}$ and $X_{d}$.

$$
\begin{aligned}
& U_{1}\left(\ell_{e}+\mathcal{L}_{d}\right)=\beta_{e} * U_{e}\left(\ell_{e}\right)+\beta_{d} * U_{d}\left(\mathcal{L}_{d}\right)=\beta_{e} \\
& U_{1}\left(\mathcal{L}_{e}+\ell_{d}\right)=\beta_{e} * U_{e}\left(\mathcal{L}_{e}\right)+\beta_{d} * U_{d}\left(\ell_{d}\right)=\beta_{d}
\end{aligned}
$$

At any given time interval subject to demand charge evaluation, the amount of cost derived from the demand charge is considerably higher than the cost of energy charge. We know that $p_{e} \ll p_{d}$. It can be inferred from this that a combination of $\left\{\mathcal{L}_{e}, \ell_{d}\right\}$ is preferred to $\left\{\ell_{e}, \mathcal{L}_{d}\right\}$, since the demand charge is the prominent factor in total cost. A low demand charge preferred to a low energy charge.

$$
\begin{array}{ll} 
& \left\{\ell_{e}, \mathcal{L}_{d}\right\} \prec\left\{\mathcal{L}_{e}, \ell_{d}\right\} \\
\text { or, } \quad & U_{1}\left(\ell_{e}+\mathcal{L}_{d}\right)<U_{1}\left(\mathcal{L}_{e}+\ell_{d}\right) \\
\text { or, } \quad & \beta_{e}<\beta_{d}
\end{array}
$$

We select $\beta_{d}=0.7$ and $\beta_{e}=0.3$ for the purpose of simulation in this dissertation, although these values may be different based on various pricing structures. The relative difference between energy and demand charges in different pricing schemes 
can affect these values. Also, the notion of the convex combination of energy and demand charge is only relevant to situations where demand charge is applied. In all other cases, $U_{1}(X)=U_{e}\left(X_{e}\right)$.

\subsubsection{Additive Multi Attribute Utility}

A multi-attribute utility function is a combination of the individual attribute value/utility functions weighted by some scaling constants. In determining the multiattribute utility function for different households these scaling constants will differ according to their socio-economic classification. We conjecture that households of different economic status will give different priorities to cost and convenience/comfort. When a decision has to be made regarding the selection of attributes under uncertainty, we have to take expected utility into account. The value of $X$ is dependent on the pricing structure being evaluated, thus it can be deterministic (e.g. TOU, CPP, etc.) as well as stochastic (e.g. RTP, demand charge, etc.). The value of $Y$ for thermostatic loads is stochastic, since the room temperature is dependent on ambient temperature which can be forecasted but is not deterministic. We generalize the expected utility by assuming one or both of the attributes is stochastic and utilize a multi-attribute utility function (MAUF). The MAUF allows us to model the preference structures of various combinations of attributes.

There are some independence conditions that we need to satisfy in order to decide the particular form of MAUF to be used. The first condition is of preferential independence, which is trivial in this case since both attributes are cardinal. An attribute is preferentially independent from all other attributes when changes in the rank ordering 
of preferences of other attributes do not change the preference order of the attribute (Thurston, 2006). This can be easily shown since both the attributes for cost and comfort are cardinal in nature. Secondly, in order to show utility independence between two attributes, we have to show that the utility of an attribute set does not change by simply changing the value of the other attribute. This is represented as $\left\{x_{a}, y_{a}\right\}>\left\{x_{b}, y_{a}\right\}$ and $\left\{x_{a}, y_{b}\right\}>\left\{x_{b}, y_{b}\right\}$. Regardless of the value of $Y$, the preference of $x_{a}>x_{b}$ always holds. We can easily say that for a fixed discomfort level $Y$, a consumer will always prefer a lower cost. This is true for the reverse as well. Given a fixed cost $X$, a consumer will always prefer a lower discomfort. Thus we can say that $X$ and $Y$ are mutually utility independent.

With mutual utility independence between the two attributes, we can represent the MAUF in the multiplicative form as shown in Equation (23). Here $k_{1}$ and $k_{2}$ are the scaling constants that determine the significance of one attribute over the other and $k$ is given by Equation (24). The multiplicative MAUF is able to represent rich preference structures, including non-linearity in attributes and their interactions.

$$
\begin{gathered}
U(X, Y)=k_{1} U_{1}\left(X, \alpha_{1}\right)+k_{2} U_{2}\left(Y, \alpha_{2}\right)+k k_{1} k_{2} U_{1}\left(X, \alpha_{1}\right) U_{2}\left(Y, \alpha_{2}\right) \\
1+k=\left(1+k k_{1}\right)\left(1+k k_{2}\right)
\end{gathered}
$$

When dealing with deterministic outcomes, the interaction part is removed by setting $k=0$, thus reducing the MAUF to a simpler additive form. However, for stochastic outcomes such as our case we need to show additive independence between attributes in order to use the additive form. Assigning scaling coefficients $k_{1}$ and $k_{2}$ to 
the MAUF for different households is intuitive. As mentioned before, different people will value cost and comfort differently, which must be reflected by the coefficients of the MAUF. A person that values cost benefits more than convenience/comfort will have $k_{1}>$ $k_{2}$. Similarly, we will have $k_{1}<k_{2}$ for someone who values his/her comfort more than cost savings and $k_{1}=k_{2}$ for neutral households who don ${ }^{e e}$ thave a specific preference on either. The classification of the population according to economic status, as discussed above in Section 3.1 can be used to set these values. However, the determination of $k$ for the interaction term in Equation (23) is not intuitive at all. For this reason we try to establish sufficient conditions to assume additive independence.

The general approach to test additive independence is based on lottery conditions. Specifically, consider two attributes $X$ and $Y$ with,$h^{e e}$ and , $\ell^{e e}$ denoting any two levels indicating high and low for each. A multiplicative MAUF is also additive if and only if the decision maker is indifferent between the following two lotteries, given all other attributes (if present) are kept constant.

$$
\begin{aligned}
& 50 \% \text { chance of }\left\{x_{l}, y_{l}\right\}+50 \% \text { chance of }\left\{x_{h}, y_{h}\right\} \\
& 50 \% \text { chance of }\left\{x_{l}, y_{h}\right\}+50 \% \text { chance of }\left\{x_{h}, y_{l}\right\}
\end{aligned}
$$

From this we can write Equation (25) as the necessary condition for additive independence. From our definition of bounded utility functions, if $X \in\left[x_{l}, x_{h}\right]$ and $Y \in$ $\left[y_{l}, y_{h}\right]$, then we have $U\left(x_{l}, y_{l}\right)=1$ and $U\left(x_{h}, y_{h}\right)=0$. This reduces the necessary condition for additivity to Equation (26). 


$$
\begin{gathered}
0.5 U\left(x_{l}, y_{l}\right)+0.5 U\left(x_{h}, y_{h}\right)=0.5 U\left(x_{l}, y_{h}\right)+0.5 U\left(x_{h}, y_{l}\right) \\
U\left(x_{l}, y_{h}\right)+U\left(x_{h}, y_{l}\right)=1
\end{gathered}
$$

The individual utility functions for a completely risk neutral household are linear, thus the combined utility function also being linear. Also, from the classification of households we assign neutral behavior to medium income households, such that they give equal priorities to cost and comfort. If we are to consider a neutral household with neutral risk attitude, we can say $U\left(x_{l}, y_{h}\right)=U\left(x_{h}, y_{l}\right)=0.5$ since a perfectly neutral behavior with equal weightage to both attributes would result in a mid-point utility when faced with two opposite extreme values of the attributes. This satisfies Equation (26), however it is difficult to show this for other types of households. Nevertheless, we can at least use this case as baseline to which we compare other cases.

For a cost favoring household, $U\left(x_{l}, y_{h}\right)>0.5$ and $U\left(x_{h}, y_{l}\right)<0.5$, since a minimum cost is desired more than a minimum comfort. Compared to the baseline case of the neutral house, the mid-point combination has slightly higher utility than 0.5 if cost is lowest and slightly lower utility than 0.5 is cost is highest, since that is the driving attribute. Similarly, for a comfort favoring household we have $U\left(x_{l}, y_{h}\right)<0.5$ and $U\left(x_{h}, y_{l}\right)>0.5$. Although the exact value of utility at these mid-points varies according to risk-averseness and cannot be generalized, we can still argue that a proportional shift on either direction of 0.5 is possible. Thus the assumption of additive utility independence for the purpose of simplifying the MAUF is not an invalid one. For example, if we had $U\left(x_{l}, y_{h}\right)>0.5$ and $U\left(x_{h}, y_{l}\right)>0.5$, then Equation (26) would never 
satisfy. Under the assumption of additivity we propose the use of Equation (27) to evaluate the multi-attribute utility, where $k_{1}+k_{2}=1$.

$$
U(X, Y)=k_{1} U_{1}\left(X, \alpha_{1}\right)+k_{2} U_{2}\left(Y, \alpha_{2}\right)
$$

The values of the scaling constants describe the nature of various households. We would want to assign a high coefficient for cost $\left(k_{1}>0.5\right)$ for cost favoring households, a low coefficient for cost $\left(k_{1}<0.5\right)$ for comfort favoring households and a mid-point value $\left(k_{1} \approx 0.5\right)$ for neutral households. However, this would require that all consumers are rational decision makers. It is possible that some consumers make irrational decisions. The classification of houses is based on the assumption that high income households are less affected by cost than low income households. But we can image the existence of anomalous behaviors. For example a high income family that cares as much or more about cost than comfort and vice a versa.

We propose the use of a skewed normal distribution with a high mean $(\sim 0.75)$ to generate values for $k_{1}$ for a cost favoring household. This ensures that the expected scaling constant is high, but a probability of getting a lower value still exists. A Johnson SB distribution with parameters $(\gamma=-2, \delta=2, \mu=0, \sigma=1)$ generate a right skewed bell curve with a mean of 0.75 (Figure 23). $\gamma$ and $\delta$ are shape parameters, $\mu$ is the location parameter and $\sigma$ is the scale parameter. Similarly, the parameters $(\gamma=2, \delta=$ $2, \mu=0, \sigma=1$ ) generate a left skewed bell curve with a mean of 0.25 , which is assigned as $k_{1}$ for a comfort favoring households. Lastly, even neutral households don ${ }^{e e} t$ always weigh their preferences as $1 / 2$ and $1 / 2$. The Johnson SB distribution with parameters 
$(\gamma=0, \delta=2, \mu=0, \sigma=1)$ generate a normal curve with mean of 0.5 as shown in Figure 23, which is assigned as $k_{1}$ for a neutral households.

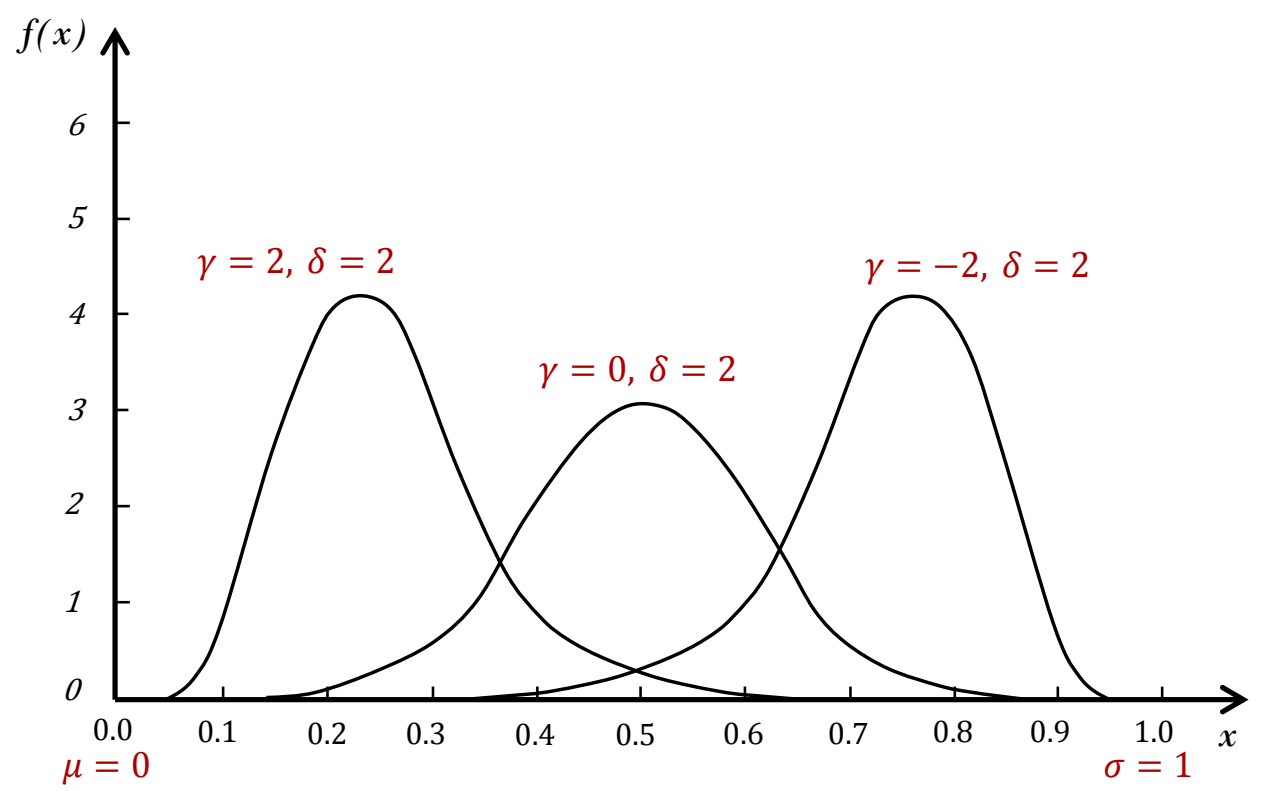

Figure 23: Johnson SB distributions with means of 2.5, 5 and 7.5 used to define $k_{1}$ for MAUF of comfort favoring, neutral and cost favoring households 


\section{CHAPTER IV METHODOLOGY}

In this section we will talk about the various methodologies used to accomplish the modeling and experimentation of consumer response in end-use energy consumption. The methods regarding consumer behavior modeling using multi-attribute utility functions and energy consumption modeling for non-thermostatic and thermostatic loads have been discussed in the previous section. Based on these models, we use discrete event simulation to study the response behavior of a population to variable pricing structures. A valid simulation model will help us to understand population behavior (system level) under various scenarios as a result of a collective response of individual households. Sensitivity analyses on alternative scenarios with different pricing mechanisms can be done to find influential parameters for changes in consumer behavior. We developed the simulation model using an object-oriented simulation package, Simio (Kelton et al., 2010). In this section we explain the key elements of the model and in Chapter 5 we will discuss experimentation and results with different system scenarios and pricing structures.

Object oriented simulation provides a method for articulating the processes of a real world phenomenon in order to build computer models. The advantage of objectoriented method lies in its ability to represent independent components of a system as objects that define their behaviors and interactions with each other. Different classes of 
objects can be modeled to represent different elements, components, sub-components, etc. of a system. Thus a complete representation of the real world system can be achieved by constructing these objects with special characteristics and logics as a network of interactions. Numerous attributes, variables, expressions and processes are used to define object characteristics and interactions. The detailed logic and processes of individual objects can be hidden so that a meaningful behavior of the model may be studied.

The primary entity in our model is a consumer, which is a representation of a household. A fixed number of households are generated at the beginning of the simulation and this sample population is maintained throughout the run. Each household is first assigned a set of characteristic parameters such as risk nature, utility functions, preferred temperature for thermal comfort as well as preferred schedules for using different appliances. As mentioned earlier, we treat non-thermostatic loads (NTL) and thermostatic loads (TCL) as independent consumption behaviors and build two separate models for convenience in this dissertation, although a combined model is simply the sum of load consumption of the two models. The behavior of the consumer towards NTL and TCL vary in our models, which is explained in detail below in Section 3.1. Figure 24 shows the different factors that affect the consumption of NTL and TCL.

The basic idea of the simulation is that a household will only consume the respective load when the expected utility is maximized. The evaluation of the utility function can be done either once a day, for deterministic pricing scenarios, or at every time interval throughout the day, for dynamic pricing scenarios. The total energy consumed by each household will be recorded accordingly when consumption occurs. In our model, we consider half hour intervals for NTL and one hour intervals for TCL as a 
time window for evaluating utility functions. The simulation time is incremental and hence we use state variables TimeOfDay, Day and Month to keep track of the respective model states.

Input factors Load model Output Load profile

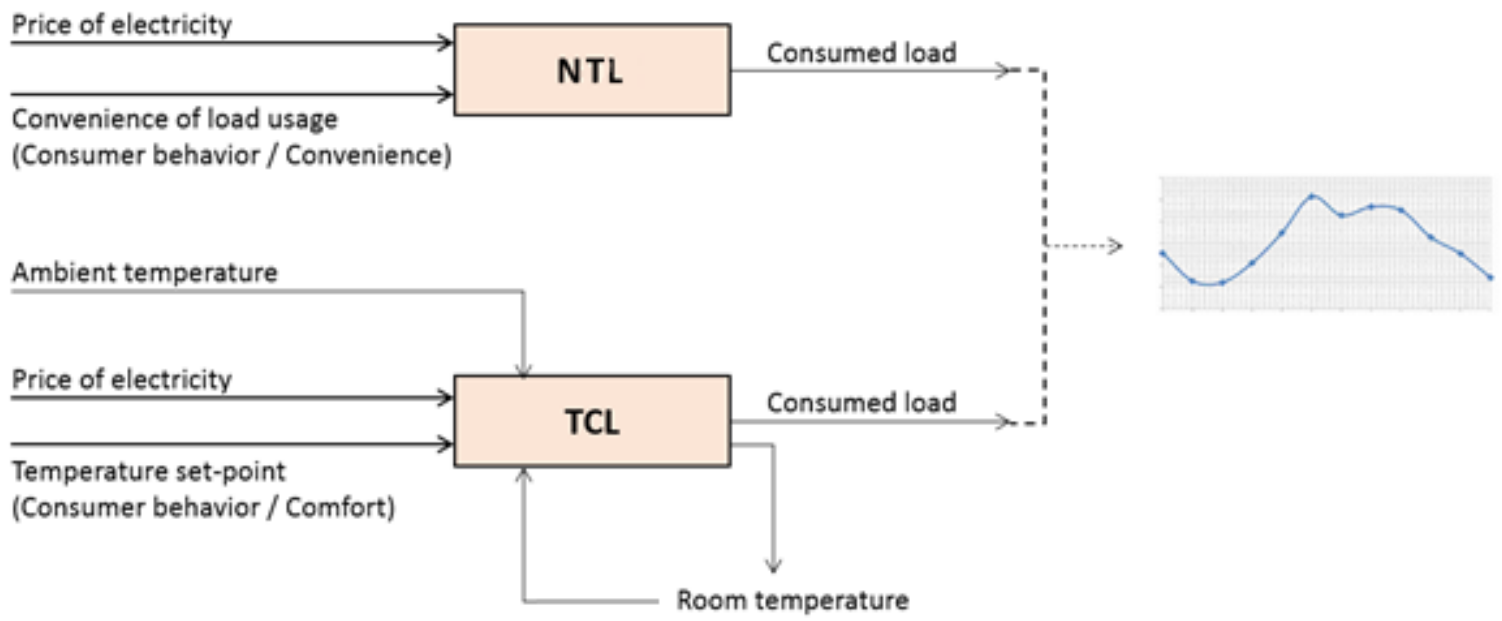

Figure 24: Different input factors that affect the consumption of NTL and TCL

\subsection{ENERGY CONSUMPTION MODELING}

Among various types of electricity consumers, a significant portion of total energy usage is from the residential sector. The main focus of this dissertation is to study the energy consumption behavior and the effect of demand response (DR) on changing consumption behaviors in the residential sector. Subsequently, the load profile over time for a given network of households will be studied to investigate the ultimate effect of DR on load leveling. Residential loads can be divided into two categories: thermostatically controlled loads (TCL) and non-thermostatic loads (NTL). TCL consists of any load that is in some way affected by ambient temperature. These types of loads are controlled by a 
thermostat that turns the appliance on or off based on a set point. NTL on the other hand are all other household appliances that don ${ }^{e e}$ have any specific control system. The load usage of NTL is thus completely dependent on the user.

Reduction of peak load can be achieved by either lowering the total demand or shifting the demand from peak periods to off-peak periods. The effect of DR is different on TCL and NTL. TCL comprises of loads like AC or heater, which cannot really be shifted to another hour. When a consumer responds to DR by turning off their AC or heater, it only results in lowering the demand at that point. Since TCL is dependent on ambient weather conditions, it cannot be shifted over a long time period. For example, it would not make sense that someone would use $\mathrm{AC}$ at $9 \mathrm{AM}$ rather than $2 \mathrm{PM}$ just because it is cheaper. Slight pseudo-shifts may occur in the form of pre-cooling, preheating or delayed consumption, but this is limited to shorter time periods. NTL on the other hand is not dependent on exogenous variables, but only the direct behavior of the consumer. Activities such as cooking, watching television, doing laundry, dish washing, etc. result in distributed load consumption based on their behavior. Unlike TCL, these appliances have varying usage patterns for different people, some of which are more flexible than others. We assume that the appliances that have flexible usage patterns are subject to load shift.

Due to the nature of consumption behavior, TCL and NTL must be modeled independently. Different approaches must be taken in studying the load changing behavior for either of the load types. In this chapter we elaborate on the energy consumption models of both types of loads and select particular appliances to include in our analysis for the remainder of this dissertation. 


\subsubsection{Non-Thermostatic Loads (NTL) Model}

Non-thermostatically controlled loads (NTL) are those that are not affected by ambient weather and are not controlled by any control system such as the dead band control of AC. Household appliances such washer, dryer, dishwasher, oven, microwave, $\mathrm{TV}$, etc. as well as lighting loads are not dependent on any exogenous variable, and thus comprise of the NTL. The Annual Energy Outlook 2015 report indicated that 57\% of the residential energy usage was from NTL. The usage pattern of NTL is mostly dependent on the behavior of the resident. However, all NTL appliances are not very flexible in their usage, for example lights, cooking range, TV, etc. But some appliances have flexible usage patterns. Load shifting behavior of NTL is more likely to happen due to potentially flexible loads.

We consider a few prototypical household appliances with potentially flexible usage to study load shifting behavior under DR. According to the US Department of Energy (www.eia.gov), the contribution to total residential energy by washer and dryer can be up to $6.7 \%$ while dishwasher can contribute up to $2.5 \%$ (EIA, 2012). Figure 5 indicates that one of the top contributors among the NTL were laundry and dishwashing appliances. Although this number doesn"t seem like much, it must be noted that the potential to change consumption pattern is important as well. Other appliances with higher load contribution, such as lighting and cooking, are less likely to see load shifts. These three appliances are considered in our model because the usage times for them is assumed to be flexible and also contributes to around $9 \%$ of the total residential load.

The average total energy consumption per person in 2009 was about 90265.87 $\mathrm{kWh}$, according to the Energy Outlook Report from EIA. We use this data as a reference 
to calculate the demand of energy for each of the appliances considered in our model. Instead of considering weekly patterns of consumption, we calculate daily load demands and assume that load consumption for these three appliances are uniform for every day. Table 3 shows the daily load demand per person for each of the appliances. When simulating a population of many households we simply multiply this number by the estimated population size.

Table 3: Annual and daily demand per person for various appliances

\begin{tabular}{|c|c|c|}
\hline \multicolumn{3}{|c|}{ Average total energy consumption per person (kWh) } \\
\hline Appliance & per year & per day \\
\hline Washer & 114.21 & 0.27 \\
\hline Dryer & 735.99 & 0.22 \\
\hline Dishwasher & 317.24 & 0.18 \\
\hline
\end{tabular}

Various studies of individual household energy consumption have shown a bimodal consumption pattern of appliances such as the ones considered in our model (Wood and Newborough, 2003). We will also use a bimodal distribution of the washer and dryer loads and assign the preferred times of usage that are uniformly distributed between $7 \mathrm{AM}$ to $9 \mathrm{AM}(30 \%)$ and $4 \mathrm{PM}$ to $8 \mathrm{PM}(70 \%)$. The dishwasher loads are set to have preferred times of usage between $8 \mathrm{PM}$ to $10 \mathrm{PM}$, usually after dinner. If the consumer uses the appliance at their preferred time, we say that they have the least inconvenience. As a response to DR, if they chose to use the appliance at a different time, then we consider that they are inconvenienced by a certain amount. This inconvenience value will be treated as a cost, similar to discomfort for TCL.

In order to quantify the inconvenience attributes, we first assign convenience values for different times of the day. The convenience of using an appliance is measured 
in two ways. Fixed convenience for all households is measured as a discrete function based on the time of the day. Depending on their schedule and habits, different people will find different times of the day more convenient or less convenient for using certain appliances. We simplify this issue by assuming that morning hours and evening hours are more convenient to an average household, since most people are at work during the day. An example of the fixed convenience is illustrated in Table 4. Convenience is also measured as a variable value depending on the preferred schedule of individual households. We set the range of values for convenience between $[0,5]$. The convenience value is 5 at the preferred time for the household to use the appliance, and it reduces by 1 for every hour it is shifted away from the preferred time. In Table 4, the variable convenience is shown at the preferred time of 16:00. The final convenience value, $C_{t}$, of using an appliance is measured by taking the higher of the fixed and variable convenience. The inconvenience is then measured as $Y=\left(5-C_{t}\right)$.

The definition of cost variables and the consumer decision making process for non-thermostatic loads (NTL) differ from thermostatic loads (TCL). We study the load consumption pattern and shifting behavior of these loads under differential pricing mechanisms separately. The basic logic behind the NTL simulation model is shown in Figure 25. At the beginning of the simulation, a fixed number of households are generated and this sample population is maintained throughout the run. Each household is first assigned a preferred schedule for using different appliances. As discussed above, we consider the usage of clothes washer, dryer and dishwasher. The household will only use the appliance when its utility is maximized. For the case of the TOU rates, the utility evaluation is done at once at the beginning of the day. 
Table 4: Example of fixed and variable convenience values over $24 \mathrm{hr}$

\begin{tabular}{|c|c|c|c|}
\hline Time & $\begin{array}{c}\text { Fixed } \\
\text { Convenience }\end{array}$ & $\begin{array}{c}\text { Variable } \\
\text { Convenience }\end{array}$ & $\begin{array}{c}\text { Total } \\
\text { Convenience }\left(C_{t}\right)\end{array}$ \\
\hline $0: 00-7: 00$ & 0 & 0 & 0 \\
\hline $7: 00-8: 00$ & 1 & 0 & 1 \\
\hline $8: 00-8: 30$ & 2 & 0 & 2 \\
\hline $8: 30-10: 00$ & 3 & 0 & 3 \\
\hline $10: 00-10: 30$ & 2 & 0 & 2 \\
\hline $10: 30-11: 00$ & 1 & 0 & 1 \\
\hline 11:00 & 0 & 0 & 0 \\
\hline $12: 00$ & 0 & 1 & 1 \\
\hline $13: 00$ & 0 & 2 & 2 \\
\hline $14: 00$ & 0 & 3 & 3 \\
\hline $15: 00$ & 0 & 4 & 4 \\
\hline $16: 00$ & 0 & 5 & 5 \\
\hline $17: 00$ & 2 & 4 & 4 \\
\hline $18: 00$ & 3 & 3 & 4 \\
\hline $19: 00$ & 3 & 2 & 3 \\
\hline $20: 00$ & 2 & 1 & 2 \\
\hline $21: 00$ & 1 & 0 & 1 \\
\hline $21: 00-24: 00$ & 0 & 0 & 0 \\
\hline
\end{tabular}

At the beginning of the simulation, each household updates their schedule of appliance usage based on their total utility. A utility evaluation step calculates the total utility for using the appliance and decides whether or not to use the appliance at the preferred time. The schedule is updated to reflect the usage time at which total utility is maximized. The entity then proceeds to a delay object, where it waits until the simulation time is advanced to its next appliance usage schedule, after which it proceeds to the respective appliance object. After finishing the appliance use, it evaluates its schedule to determine the next appliance to use and then goes to the delay step to wait for it. Note that we assume that once an appliance is started, it will be used continuously until its 
daily demand is completed. Thus, the simulation model records/updates the total energy used by the household when it starts to use an appliance.

In the Simio model, an entity class object "Household" is defined with three expression properties. When inserting different types of household entities into the model, these properties can be changed according to the different categories of households. The household entity is assigned to groups A, B or C based on the proportion given in Table 1 to indicate the different socio-economic classifications. The expression properties $U_{-} X$ (utility function of cost), $U_{-} Y$ (utility function of convenience) and MAUF (multi attribute utility function) are defined as in Section 3.2. Each group of households can have varying risk natures. For households within each group we assign the attributes alpha 1 and alpha 2 with random values from uniform distributions [-9, 0) and $[-0.4,0)$, respectively, as discussed in Section 3.2.3. The total number of households is set be 100 and the total run time for the simulation model is set to be 48 hours so as to allow one day for warm-up and to study the load profile on the second day. Since there is no stochastic variation in the cost structure, we assume that the base load profile (total load minus the three appliances) will remain constant every day. The number of replications is determined to be $n=25$ by setting a desired half-width of the $95 \%$ confidence interval for the average load to be $2 \%$ of the average load from an initial run with $n=10$.

The utility maximization algorithm used by each household to evaluate its schedule of appliance usage is imperative to the model. Each household entity is embedded with this algorithm to run independently of others. Because the TOU rate 
structure is not subject to change frequently or without notice, we can say that the customers decide their appliance schedules at once for the whole day.

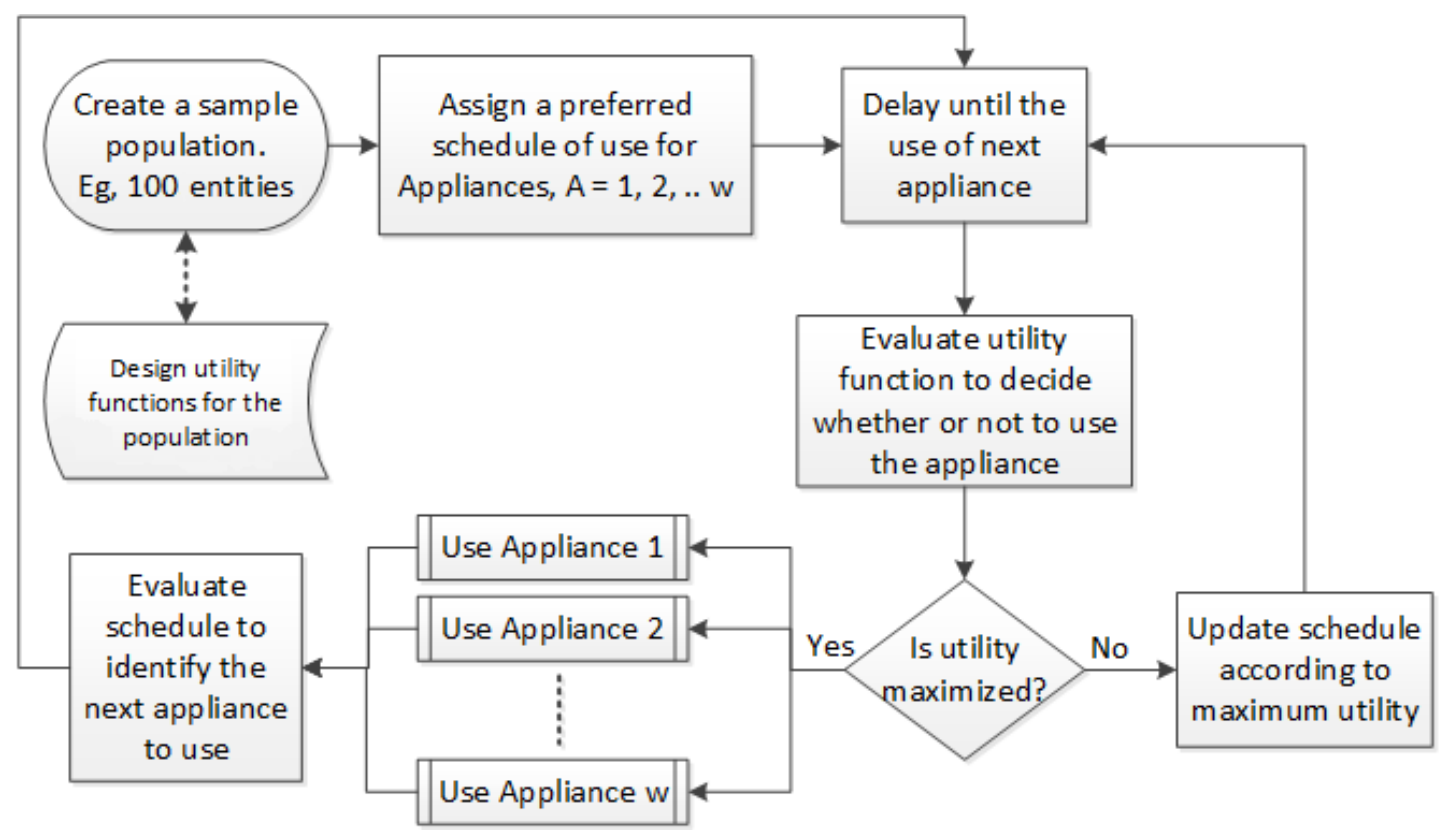

Figure 25: A framework of using multi-attribute utility function for the simulation of

NTL energy consumption scheduling

In the model, each entity will evaluate its utility function for every half-hour for the entire day. To do this we define a temporary vector variable with 48 rows (corresponding to 48 half-hour time intervals during a day), which will record the utility value at each of the time interval mentioned. Since this algorithm is embedded in the household entity, simultaneous utility evaluation is possible for all the entities without affecting each other. At the start of this process, a variable called Time is set to zero and after each iterative utility evaluation it is incremented by 0.5 . A lookup table for $X$ (cost) and $Y$ (convenience) are defined in order to call upon the corresponding values at 
different times. At each iteration the cost of using the respective appliance is estimated by executing another process and the average rate during the usage is incorporated into the MAUF. After all the elements of the temporary vector are filled, another process is executed in order to select the time corresponding to the maximum utility by using a simple bubble sort algorithm. Algorithm 1 and Figure 26 concisely illustrate the utility maximization process. If more than one maximum value is found then we choose the time closest to the preferred time of use. The model continuously monitors the total energy being used in the system by recording the energy drawn by each appliance into a global variable called TotalEnergy.

Algorithm 1: Utility Maximizing for Each Household

Step 0: Initialize vector temp $[\mathrm{i}]=0$, for all $\mathbf{i}$

Step 1: Initialize $i=1$, Time $=0$

Step 2: Set $X=\operatorname{Rate}[$ Time], $Y=$ Inconvenience[Time]

Step 3: Evaluate the utility for these variables, temp $[\mathrm{i}]=\operatorname{MAUF}(\mathrm{X}, \mathrm{Y})$

Step 4: $i=i+1$ and Time $=$ Time +0.5

Step 5: If $i>48$ goto Step 6, Else goto Step 2

Step 6: Find $j$ such that temp[j] $=\max \{\operatorname{temp}[1]$, temp[2], .. , temp[24] $\}$

Step 7: Assign new start time for appliance $=(\mathrm{j} / 2)-0.5$ 


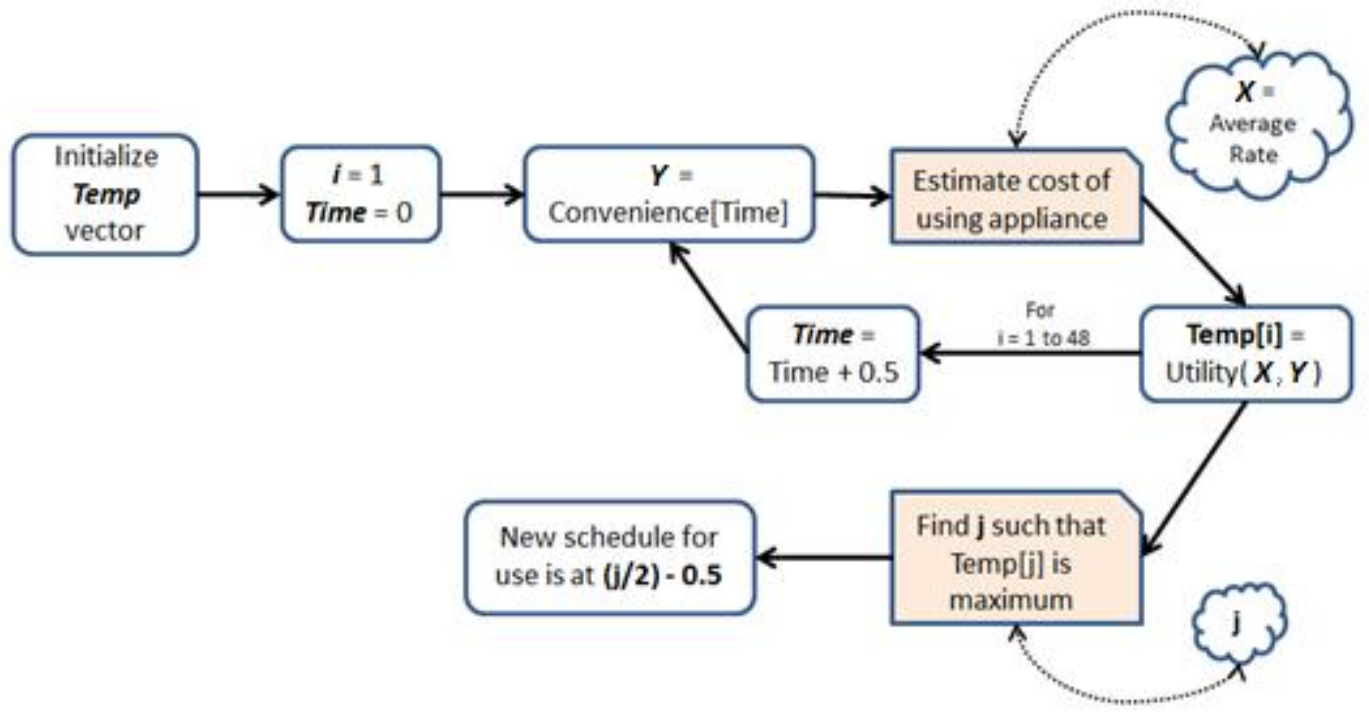

Figure 26: Utility maximizing algorithm embedded in the Household entity

The global variable TotalEnergy requires the information on the wattage and duration of use, every time an appliance is being used. We consider three appliances: washer, dryer and dishwasher in our study of non-thermostatic loads. Each household has a certain preferred schedule for each appliance. This preferred schedule is only an estimation based on a recorded example of residential demand profile by Wood and Newborough (2003) and assumptions of a typical household activity. Also based on household size (i.e., the number of people in the household), the duration of these appliances $^{\text {ee }}$ usage is set for each household. The duration of usage for each appliance is estimated by using the average demand for energy per person and the proportion of energy delivered by end-use. We have a bimodal distribution for each appliance ee usage pattern. In particular, we assign $30 \%$ of the entities to use the washer and dryer during morning hours and remaining during evening hours. For the morning use, we set a preferred schedule of $\operatorname{Uniform}(7,9)$ for clothes washer and $\operatorname{Uniform}(8,10)$ for dryer. The 
evening schedule is set as $\operatorname{Uniform}(16,18)$ for clothes washer, $\operatorname{Uniform}(17,19)$ for dryer and Uniform $(20,22)$ for dishwasher. The starting times are expressed in a 24 hour format. From the residential energy consumption survey (RECS) data for 2001, 2005 and 2009 (EIA, Residential Energy Consumption Survey), we estimated a range of values for total energy consumption of each type of appliance per household. The duration of use for different appliances and different households is set as a uniform random variable as compiled in Table 5 .

Table 5: Appliance usage durations for various household sizes

\begin{tabular}{|c|c|c|c|}
\hline No. of occupants & $\mathbf{1}$ & $\mathbf{2}$ & $\geq \mathbf{3}$ \\
\hline WasherTime $(h r)$ & Uniform $(0.12,0.27)$ & Uniform $(0.24,0.54)$ & Uniform $(0.48,1.08)$ \\
\hline DryerTime $(h r)$ & Uniform $(0.13,0.22)$ & Uniform $(0.26,0.44)$ & Uniform $(0.52,0.88)$ \\
\hline DishwasherTime $(h r)$ & Uniform $(0.1,0.18)$ & Uniform $(0.2,0.36)$ & Uniform $(0.4,0.72)$ \\
\hline
\end{tabular}

A special object named "Appliance" is defined to represent an appliance. Two properties are defined for this object, namely Wattage and ProcessingTime. Wattage is a numeric property and is required to have a numerical value whereas ProcessingTime is an expression property and can take the value of a referenced variable. Three "Appliance" objects are included in the model and named Dryer, Washer and Dishwasher. The wattages for each of these are uniformly distributed within a typical range of appliance rating values (www.energy.gov). Dryer wattage is Uniform(1800, 5000) Watts, washer wattage is $\operatorname{Uniform}(350,500)$ Watts and dishwasher wattage is $\operatorname{Uniform}(1200,2400)$ Watts. The ProcessingTime for each of the appliances are set according to each household 's demand for the appliance in its preferred schedule. 
A household entity may use multiple appliances at any given time. We model this by using a copy of the entity to seize the appliance, while the original entity is sent back to evaluate the delay for the next appliance according to its schedule. The copy of entity is used to update the global variable TotalEnergy and then it is destroyed after its intended use. By doing so, we always maintain the population of the system since all the copies are destroyed after their use and all the originals are routed back into the model. The household agent is embedded with various algorithms to make decisions based on information from the model. These algorithms are defined as sets of processes, which are triggered by the event properties such as EvaluateNextUse, EvaluateUtilityFunction, etc. Event properties are basically messages that can be sent to the entity in order to trigger various processes based on model conditions.

\subsubsection{Thermostatically Controlled Loads (TCL) Model}

Thermostatically controlled loads (TCL) are defined as the ones that are a function of a thermal set-point and also dependent on ambient temperature, such as airconditioner, space heater, water heater, etc. The Annual Energy Outlook 2015 report indicated that $43 \%$ of the residential energy usage was from TCL, out of which $13 \%$ was attributed to air-conditioning and $12 \%$ to heating loads in 2014 . With a combined share of $25 \%$, HVAC systems are the major contributors of electric load. In this dissertation, we will focus on the modeling and response of HVAC load under the TCL group. We generalize the energy consumption model to include both heating and cooling modes of the HVAC system. The main independent variable that defines load consumption for different households is their preferred thermal set-point value. Although we are studying 
the DR response of TCL for only the HVAC loads, the approach will be general enough that similar models for other TCL can be developed as well.

The use of HVAC is to regulate the internal condition of a house or building. Based on a desired set-point value, the AC or heating unit turns on or off when the inside temperature crosses a fixed dead band around the set-point value. The HVAC load is basically the energy consumed by the unit during the "on" periods. There are a lot of variables that affect the operation of the HVAC system. The set-point, inside temperature and relative humidity are the main determinants of the on/off cycles of the system, but a lot of other variables have an effect on these values. The ambient weather conditions including outside temperature, humidity, wind speed, solar irradiation, cloud cover, etc. have a direct effect on the inside conditions. The heat transfer between the outside and inside environment is dependent on the construction of the house. Insulation capacity of walls, ceilings and floors combined with the number of windows and their thermal conductivity play a big role. Other factors such as, the number of rooms, internal area, numbers of occupants, etc. also contribute to the thermal capacity of the house.

The amount of energy used by the HVAC unit is dictated by the thermal dynamics of the house. The relationship between the external conditions and internal conditions plays a vital role in determining the use of cooling or heating energy. The thermal dynamics of a house is an essential part of load consumption study of TCL and can be modeled in various ways. The research in thermal modeling is quite extensive but some of the major methods used in thermal dynamics are listed below.

- First principle based models

- Thermal electric circuit equivalent models 
- State space representations

- Models using thermal mass of buildings

- Linear and non-linear models

First principle based thermal models are built using the fundamental physical properties of the environment being modeled. It utilizes the thermal capacity of air along with thermal capacitance, conductance and resistance of building materials to represent the heat flow as a differential equation over temperature. Balan et al. (2011) for example, used a simplified thermal model consisting of heat balance equations for two distinct nodes, air and wall. Kundu et al. (2011) also use a differential equation form to model the change in inside temperature. Another fundamental method of modeling thermal dynamics is by using an equivalent electric circuit model. In this method the thermal properties and heat flow are represented using an equivalent electric circuit with corresponding electric properties (e.g., Katipamula and $\mathrm{Lu}$, 2006). A state space representation with parameter identification is also used to model thermal behavior (e.g., Xu and Deng, 2013 and Avci et al., 2013). Braun (2003) used the change in building thermal mass as a method to represent energy consumption. Aside from these methods, simple linear models are also used to represent thermal dynamics. For example, Li et al. (2011) used a simple linear form where inside temperature is the output variable and the input variables include temperature gradient between inside and outside space, energy usage of AC and the thermal parameters of the building. Wen and Burke (2013) used a second order autoregressive model with exogenous input (ARX) to define the thermal model of a house. They reduced the model to a linear form for simplicity and identified the coefficients using recursive least square method. 
Even though the importance of a building "s sthermal dynamic model is evident for the study of load consumption behavior, the focus of this dissertation is on the behavioral change of the consumer. Similar to the model used by Li et al. (2011), we use a simple linear model to represent an approximate thermal behavior of a house for the purpose of this dissertation. As shown in Equation (28), the room temperature of the next time period, $T_{t+1}$, is given by a function of current room temperature, $T_{t}$, gradient between current inside temperature and next time periodes soutside temperature, $T_{t+1}^{o}-T_{t}$, and energy consumed by HVAC unit, $q w_{t}$. The power rating of the HVAC unit is denoted by $q$ and the total "on" time during current time period represented as a fraction $[0,1]$ is denoted by $w_{t}$. If the HVAC unit is completely off then $w_{t}$ will be 0 , and if it is "on" for the entire time period, $w_{t}$ will be 1 . The coefficients of the model will be different for every house and they define its thermal characteristics. The identification of these parameters is done by using data collected for 26 homes and will be discussed in detail in Section 4.2.1.

$$
T_{t+1}=\lambda^{\prime}\left(T_{t}\right)+\lambda\left(T_{t+1}^{o}-T_{t}\right)+\gamma\left(q w_{t}\right)
$$

Another input variable that dictates the amount of energy usage of HVAC is the set-point temperature. Based on the set-point selected by a user, the AC signal $w_{t}$ is selected such that the room temperature, $T_{t+1}$, approaches this value. Each consumer has a particular preferred temperature at which they feel the most comfortable. Although a generally accepted comfortable temperature is around $70-72{ }^{\circ} \mathrm{F}$, thermal comfort is a relatively subjective matter. Some people may feel most comfortable at $65^{\circ} \mathrm{F}$ while some 
may prefer $75^{\circ} \mathrm{F}$. An empirical study on actual inside temperatures for test houses will be used to generate a distribution of preferred temperatures. We can also observe that the average temperature of a house is much lower during winter than in summer, which leads us to believe that the preferred temperature for a person is also dependent on the weather conditions. The concept of actual vs absolute preferred temperature has already been discussed in Section 3.2.3.1, dealing with this variation in set point.

The motivation of this dissertation is in the modeling of consumer response to DR programs. We have established the need for considering the utility of comfort along with cost saving behavior. When considering TCL, we include thermal comfort as a desired factor along with financial incentive. Similar to the cost variable, we consider the cost of discomfort to be included in the decision making process of the consumer. The discomfort attribute is measured as the amount of deviation from the preferred temperature experienced by the consumer as result of the change in energy consumption behavior.

The control of thermostatic loads differs from that of non-thermostatic loads, since their consumption is continuous while non-thermostatic loads tend to be consumed at discrete times. Based on a temperature set-point, the load usage depends greatly on the thermal properties of the house. Well insulated homes will have a much lower TCL consumption as compared to poorly insulated homes, since the loss of thermal energy needs to be compensated. We study the energy usage behavior of HVAC systems in our study. The decision regarding the use of cooling or heating cycle of HVAC is dependent on current room temperature, ambient temperature and the thermal dynamics of the house. 
The essence of our simulation is in the varying characteristics of individual entities and how they react to DR pricing and ambient temperature within the model. Depending on the deviation of inside room temperature from the preferred temperature, consumers will feel certain discomfort, which they trade-off against electricity cost via utility maximization. The utility maximization is the central optimization problem for the decision maker to determine appropriate HVAC consumption at every time interval. The decision making process utilizes a model predictive control (MPC) mechanism which is discussed in detail in Section 4.2. Figure 27 illustrates the basic framework used for modeling the HVAC consumption based on ambient temperature and pricing information. The generation of a sample population and their classification is similar to the NTL model. The expression properties $U_{-} X e$ (utility function of energy charge), $U \_X d$ (utility function of demand charge), $U_{-} Y$ (utility function of comfort) and MAUF (multi attribute utility function) are defined as in Section 3.2. The various electricity rates such as $p \_e$ (energy charge), $p \_d$ (demand charge), OnPeak_Pe (on-peak energy charge), OffPeak_Pe (off-peak energy charge), etc. are defined as model parameters.

In the TCL model, assigning a base load to the household entities is important, since it determines the upper and lower bounds of the utility functions. This is already explained in Section 3.2.3 with the base load estimation from 26 test homes shown in Figure 15. Each group of household entities (based on socio-economic classification) is also divided into three size levels based on the occupancy (Table 2). The different base loads in Figure 15 are assigned to the corresponding entity of the respective size. The values of $l_{-} \min$ and $l_{-} \max$ are defined as follows. 
- Low occupancy: $l_{-} \min =0.2629 \mathrm{~kW} ; l_{-} \max =1.2928 \mathrm{~kW}$

- Med occupancy: $l \_\min =0.9382 \mathrm{~kW} ; l_{-} \max =1.5492 \mathrm{~kW}$

- High occupancy: $l_{-} \min =1.1162 \mathrm{~kW} ; l_{-} \max =2.5042 \mathrm{~kW}$

Similarly, the maximum power rating $(\mathrm{kW})$ for the HVAC during cooling (AC) and heating cycles are defined as follows. These values were estimated using the sub-metered data for the 26 test homes in Glasgow, KY for 2014. Depending on the thermal cycle required, the appropriate value of $q$ is used.

- Low occupancy: $q_{-} A C=$ Uniform $(2,4) ; q_{-}$Heat $=$Uniform $(2,7)$

- Med occupancy: $q_{-} A C=$ Uniform $(3,5) ; q_{-}$Heat $=$Uniform $(5,10)$

- High occupancy: $q_{-} A C=$ Uniform $(4,6) ; q_{-}$Heat $=$Uniform $(8,13)$

The utility functions for the household entities are defined by Equations (29), (30) and (31). These are simply Equation (7) replaced by the corresponding upper and lower bounds. The parameter $D t$ in Equation (31) is assigned for each household as the maximum allowed deviation from preferred temperature that causes the minimum utility. The estimation of this value is given with other thermal parameter identifications in Section 4.2.1.

$$
\begin{gathered}
U_{-} X e=\frac{e^{a l p h a 1_{-} e * X e}-e^{a l p h a 1_{-} e *\left(l_{-} \max +q\right) * O n P e a k_{-} P e}}{e^{a l p h a 1_{-} e *\left(l_{-} \text {min }\right) * O f f P e a k_{-} P e}-e^{a l p h a 1_{-} e *\left(l_{-} \text {max }+q\right) * O n P e a k_{-} P e}} \\
U_{-} X d=\frac{e^{a l p h a 1_{-} d * X d}-e^{a l p h a 1_{-} d *\left(l_{-} \max +q\right) * O n P e a k_{-} P d}}{e^{\text {alpha1 } d *\left(l_{-} \text {min }\right) * O f f P e a k_{-} P d}-e^{a l p h a 1_{-} d *\left(l_{-} \text {max }+q\right) * O n P e a k_{-} P d}} \\
U_{-} Y=\frac{e^{a l p h a 2 * Y}-e^{a l p h a 2 * D t}}{1-e^{a l p h a 2 * D t}}
\end{gathered}
$$


The risk averseness of different households in the above utility functions are assigned as alpha1_e $=$ Uniform $(-8,0) \neq 0$, alpha $1_{-} d=$ Uniform $(-0.08,0) \neq 0$ and alpha $2=\operatorname{Uniform}(-0.8,0) \neq 0$ based on appropriate risk premium values. Although we decided to use 0.7 as the coefficient of demand charge in the utility of cost (Equation 17 in Section 3.2.3.2), we assign the parameter beta2 for the household entities as shown in Equation (32), where $M$ is a very large number. This is a general representation, since beta 2 will be zero if $p_{-} d$ is zero, thus reducing the utility of cost to just $U \_X e$. The utility of total cost is defined as Equation (34).

$$
\begin{aligned}
& \text { beta } 2=0.7 *\left(1-e^{-p_{-} d * M}\right) \\
& \text { beta } 1=(1-\text { beta } 2) \\
& U_{-} X=\text { beta } 1 * U_{-} X e+\text { beta } 2 * U_{-} X d
\end{aligned}
$$

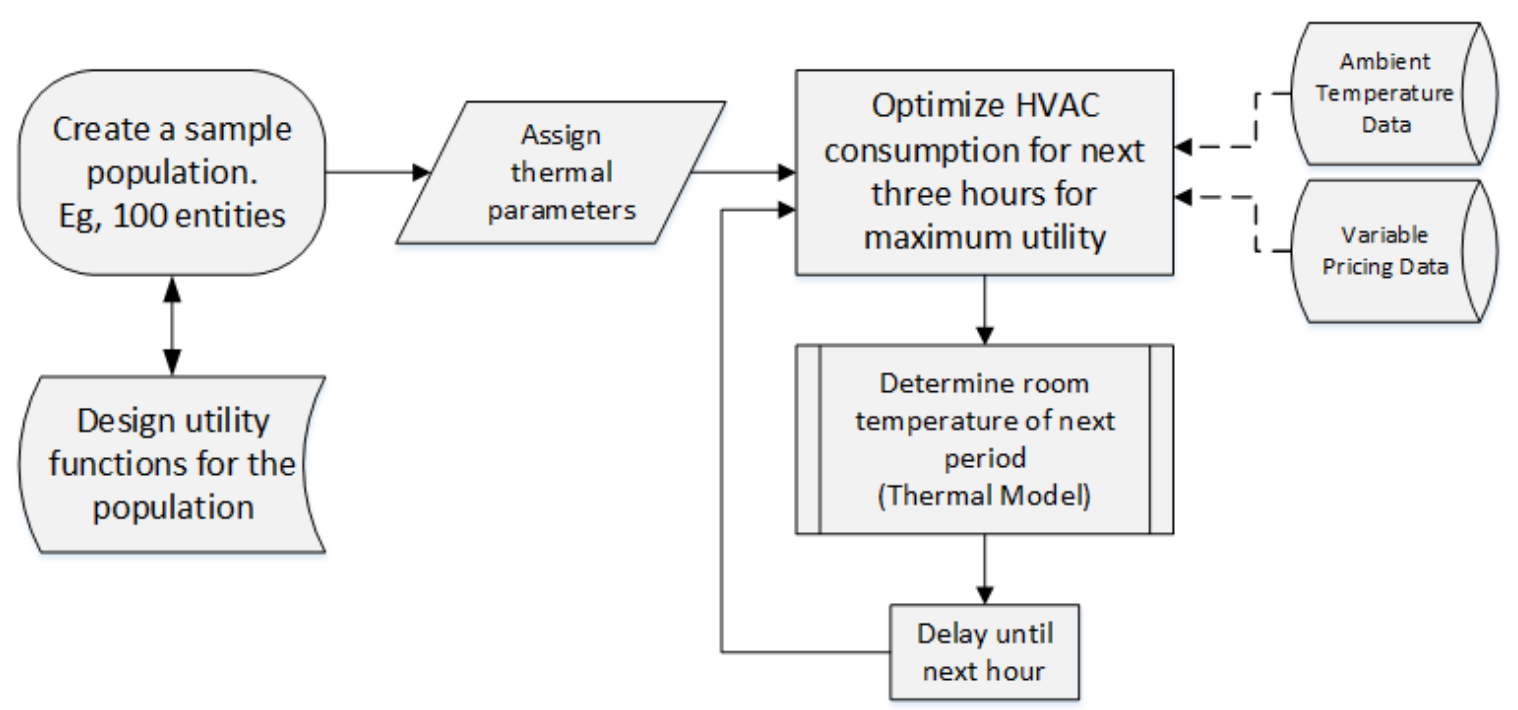

Figure 27: A framework of using multi-attribute utility function to optimize HVAC load for the simulation of TCL consumption 
Unlike the NTL model, load consumption is a function of ambient temperature variations in the TCL model, thus requiring weather information throughout the simulation run. The decision making process of the consumer is based on the information of electricity pricing (rates) as well as a forecast of outside temperatures. After a load consumption decision is made, the room temperature of the household needs to be updated according to actual outside temperature and the thermal model of the house. The parameter identification for the thermal models is presented in Section 4.2.1. We use the actual weather information for Glasgow, KY in 2013 as the outside temperature. We simulate the forecasted temperature values by randomly changing the actual values within $2^{\circ} \mathrm{F}$. Since the decision making process is only defined for a three hour interval, we assume that temperature forecast doesn"et fluctuate more than $2^{\circ} \mathrm{F}$.

The forecasts of ambient temperature are included in a lookup table as a function of the time of day. Two vectors called Temperature and Forecast keep track of the respective temperature information for the current day. To assist in the correct temperature assignments, a separate process is initiated at the beginning of each day $($ TimeOfDay $=0)$ which updates these vectors. Depending on the value of month and day, appropriate information from data tables are retrieved and replaced in the Temperature and Forecast vectors. Lookup tables, which return certain values as a function of the time of day, are used to model the stochasticity of the input ambient temperature. The decision intervals for the households are set to be one hour, and at every time step the household uses data from the Forecast vector and pricing information to maximize the expected utility. 
In order to reduce computational time, the household object is embedded with an optimizing algorithm that uses recognition heuristic and bi-section search which will be discussed in detail in Section 4.3. The algorithm optimizes the objective function of the MPC to a desired level of accuracy instead of solving the complex non-linear program (NLP) for its optimal value. This is also supported by the principle of bounded rationality as discussed before, which states that consumers usually make approximate decisions based on limited information. After the household finds its optimal HVAC signal, the room temperature of the house is updated according to actual outside temperature and the thermal model of the house. The household entity then waits in a delay step until the simulation time is incremented to the next hour. The entity is routed back to the previous module where it repeats the optimization process for the next hour. We are not destroying any entities in this model, thus the initially created population is maintained throughout the simulation. Various essential parts of the TCL model are described in detail in the following sections.

\subsection{MODEL PREDICTIVE CONTROL OF TCL}

Model predictive control (MPC) is a widely used tool in control theory that uses a receding horizon approach to optimize complex stochastic systems. The fundamentals of MPC lies in obtaining appropriate control actions, at every sampling step, for a particular system by optimizing a finite time problem based on the predictions of stochastic variables that affect the desired output. The MPC method has been in use since the $1980^{\circ} \mathrm{s}$ as an advanced process control tool in the chemical industry where complex dynamic systems with multiple stochastic variables. The benefit of this method is that a 
system is controlled by finding optimal actions for the current timeslot while taking into account the variability of future timeslots as well. The application of this method has spread to various other fields with complex dynamic structure such as power systems, automotive industry, food industry, etc. The objective function that is central to the MPC method is usually termed as a "cost function" whose general form is given by Equation (35). In this cost function, the first part aims to reduce the output error or deviation from reference point and the second part to reduce extreme variation in the input variable. The variables $x_{i}, r_{i}$ and $u_{i}$ denote the output, reference and input variables at time $i$. The weighting coefficients $w_{1}$ and $w_{2}$ give relative importance to each of these parts.

$$
\text { Minimize } J=w_{1} \sum_{i=1}^{N}\left(r_{i}-x_{i}\right)^{2}+w_{2} \sum_{i=1}^{N} \Delta u_{i}{ }^{2}
$$

MPC provides appropriate control actions, at every sampling step, for a particular system by optimizing a finite time problem based on the predictions of stochastic variables that affect the desired output. The main idea of MPC is to utilize a mathematical model of the process to predict the future behavior of the system over a prediction horizon and to compute control actions by optimizing a cost function depending on these predictions subject to various constraints. MPC is based on iterative, finite horizon optimization of a plant model. An appropriate sampling time is selected depending on the nature of the system, at which some control inputs are to be implemented. At a particular time instance $k$ an objective function is minimized or maximized for a relatively short time horizon in the future $[k, k+p]$, as shown in Figure 28. From the series of optimal control actions obtained for this prediction horizon, only the first input is implemented. 
The plant state, or system model, is sampled again from the actual output measured and updated accordingly. The calculations are then repeated starting from the new current state $k+1$.

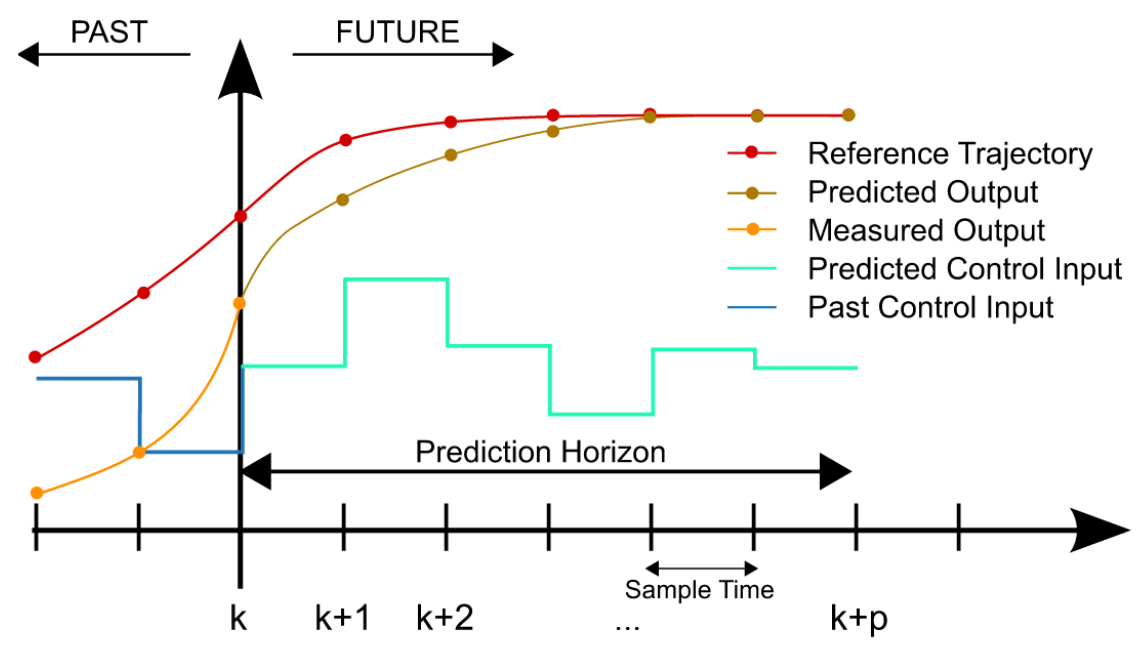

Figure 28: Basic principle of model predictive control (MPC)

In recent years, MPC has gained much popularity in controlling thermostatic loads (TCL) since it can closely match a reference signal by predicting future conditions and optimizing on a rolling horizon basis (see e.g., Avci et al., 2013; Xu and Deng, 2013). Most of the literature in this field studies the control of energy consumption, by changing AC control signals or by controlling the thermostat set-point, based on a system model that captures the thermal dynamics of the house. The use of MPC to control the thermostat settings of residential households in exchange for cost benefits provides a valid method of direct load control but it gives the authority of set-point change to the service provider, neglecting possible overrides. We are interested in the behavior of the consumer as the controller. The consumers will themselves determine their optimal consumption according to changing electricity prices, ambient temperature and thermal 
comfort. We propose the use of MPC mechanism to model the consumer behavior, with the minimizing cost function being replaced by the maximizing utility function. The design of pricing structures can be studied in response to consumer behavior.

Let us consider a sampling step of one hour to model energy consumption control behavior. Figure 29 illustrates the basic outline of the model predictive control mechanism applied to simulate the decision making process regarding TCL consumption. At any given time period $t$, the consumer is faced with the decision of the amount of HVAC consumption $w_{t}$. The dilemma herein lies in the trade-off between the cost of such consumption and the comfort achieved from the resulting room temperature in the next period $T_{t+1}$. As we know, the room temperature for the next period is affected by the current room temperature $\left(T_{t}\right)$, amount of HVAC used $\left(w_{t}\right)$, external temperature $\left(T_{t}^{o}\right)$ and random effects from other unobserved factors. In the simulation model each household is provided with a thermal model for this purpose.

At any given time period $t$, the consumer looks ahead a few hours to determine the sequence of optimal HVAC consumption. This is called the prediction interval denoted by $\{t, t+k \delta\}$, where $k$ is the number of time periods to consider and $\delta$ is the sampling step size (one hour). The outside temperature cannot be known in advance. Forecast of weather is available to the consumer, and although quite precise within a short period, the actual outside temperature may vary in reality, especially when the prediction interval is large. The cost of using electricity is also considered to be uncertain when using dynamic pricing schemes or demand charge based pricing models. The consumer thus has to make a forecast of these two variables, indicated in Figure 29 by the vectors $\vec{p}$ and $\overrightarrow{T^{o}}$, for each timeslot of the prediction interval $i=[t, t+k \delta]$. Given these 
input vectors and current room temperature $T_{t}$, the optimal energy schedule $\vec{w}$ is obtained by maximizing the consumer"s total utility over the interval $\{t, t+k \delta\}$. The maximum utility accounts for the cost of electricity as well as thermal comfort as defined by the multi attribute utility function. From this optimal set of consumption values (control actions) the HVAC consumption for the first period $w_{t}$ is then implemented. The actual cost of energy usage is calculated by applying the actual pricing value $p_{t}$ to $w_{t}$. Also, the room temperature for the next time period is then evaluated using the thermal model again, thus repeating this cycle on a rolling basis.

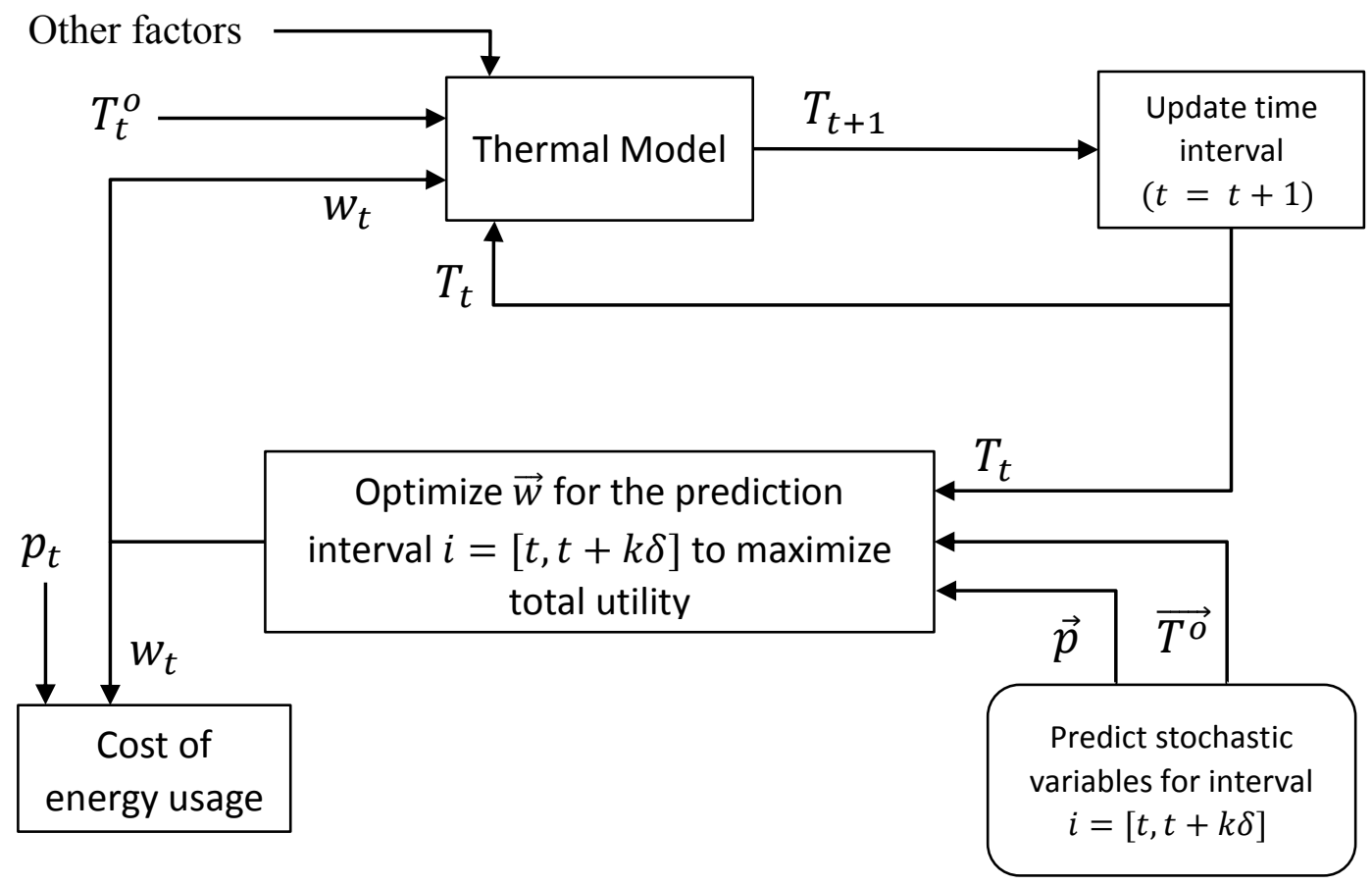

Figure 29: Outline of the model predictive control (MPC) mechanism used to simulate utility driven TCL consumption behavior 
Classical MPC models employ a feedback control system using observed output values to estimate the system dynamics model, also referred to as the predictor model. This is usually represented as a state space model which is updated after every new observation. This allows for an up to date representation of the actual system, called the plant model. The thermal model shown in Figure 29 may be confused with the plant model. However, the thermal model in our simulation is developed using estimated thermal parameters and thus is considered to be constant and known. Since the application of MPC in our context is to the consumer decision making process, the predictor model is the representation of the cognitive process of the consumer. When faced with variable pricing, the consumer initially estimates the electricity prices but as more and more information is gathered, this estimation is expected to be more accurate. For example, the cost of electricity due to demand charge is an uncertain value dependent on the state of the system being at peak. There exists a probability of the prediction interval being at peak given the current state of the system. As more data is gathered regarding this state change, this probability becomes more and more stable. Theoretically, the steady state probability that a given time period will be at peak will converge over time, with enough information. This is in fact analogous to the predictor model of the classical MPC. We use a conditional markov chain model to represent the state change probabilities, discussed in detail in Section 4.4.

\subsubsection{Thermal Model Parameter Identification}

In the interest of TCL consumption modeling it is important to carefully consider the thermal dynamics of a house. As mentioned earlier, the amount of energy consumed 
by TCL is directly affected by the thermal property of a house in regards to the conservation or loss of thermal energy. Various elements such as building material, insulation, thermal capacitance, thermal resistance, number of windows and doors, etc. affect the heat flow between the outside and inside environment for a house. The behavior of TCL consumption is modeled using MPC mechanism which includes an optimization step, a prediction step and the thermal model as the main components (Figure 29). An important component of the MPC process, when dealing with the control of temperature is the thermal model. The optimization of HVAC input signals for the control process is based on the thermal dynamics as well. When optimizing $\vec{w}$ for a multiple timeslot prediction interval, the estimated room temperature for each timeslot $\{t+1, t+k \delta\}$ is based on the thermal model.

There are various ways to model a thermal system such as state space model with parameter identification, first order differential equations, thermal electric circuit representation, and simplified linear model. Since the scope of this research does not necessarily deal with highly precise thermal dynamics we select a simplified linear model. The relationship between the outside temperature, inside temperature and HVAC consumption can be represented as a black-box model using a linear regression with the coefficients identified from real data. The linear dynamic model given in Equation (36) can be used to model the inside temperature of a room as a function of ambient temperature and energy consumed by the HVAC unit ( $\mathrm{Li}$ et al., 2011). The room temperature for the next time period $T_{t+1}$ is a linear function of current room temperature $T_{t}$, the temperature gradient between outside and inside environment $\left(T_{t+1}^{o}-\right.$ $\left.T_{t}\right)$, and the HVAC energy consumption $\left(q w_{t}\right)$. The regression coefficients $\lambda^{\prime}, \lambda$ and $\gamma$ 
are the thermal parameters than define the nature of each house. $\lambda^{\prime}$ and $\lambda$ denote the thermal efficiency or insulation capacity while $\gamma$ represents the efficiency of the HVAC unit. Although Li et al. (2011) uses $\lambda^{\prime}=1$, we leave the estimation of this value to data fitting as well. The HVAC unit efficiency will be $\gamma>0$ for heating cycles and $\gamma<0$ for cooling cycles.

$$
T_{t+1}=\lambda^{\prime}\left(T_{t}\right)+\lambda\left(T_{t+1}^{o}-T_{t}\right)+\gamma\left(q w_{t}\right)
$$

To represent varying thermal behavior of a population, we estimate the coefficients of Equation (36) using data collected for 26 test homes in Glasgow, KY 2014. These 26 homes were equipped with sub-metering capabilities, thus providing data $\log$ for separate appliances as well as inside and outside temperatures. A graphical example of one of the test houses (HOME 01) showing all relevant data records is shown in Figure 30. From this data, the input and output variables were defined accordingly and a linear regression analysis was conducted for each test house. Since the HVAC load records both heating and cooling cycles, we separated the data into two parts based on the inside temperature $(T)$ and consumer ${ }^{\text {ee }}$ s preferred temperature $\left(T_{p}\right)$. For simplicity, all data points with $T<T_{p}$ are considered as heating loads and all data points with $T>T_{p}$ are considered as cooling loads. The estimation of $T_{p}$ for each house was done as explained in Section 3.2.3.1 (Figure 18). Thus for each test house we gathered the relevant variables for heating and cooling loads and fit multiple linear regression models. The resulting regression coefficients and their corresponding p-values were recorded, an example of which is shown in Table 6. The p-values for the regression coefficients for 
most of the houses were found to be very low $(\approx 0)$, indicating a high statistical significance.

Some coefficients for a few houses that resulted in $p$ value $>0.05$ were omitted from further analysis. Also the data for heating cycles were not usable for all homes. It was observed that about half of the test homes showed very low HVAC or heating loads during winter time. One possible explanation for this is that these homes are gas heated and thus show a minimum HVAC consumption during winter even though the internal temperature is maintained at a comfortable level. From the 26 homes data, thermal parameters for cooling cycle was fit for 21 homes while only 10 homes were selected for heating cycle. These estimated coefficients are presented in Table 7.

\section{Load Profile: HOME 01}

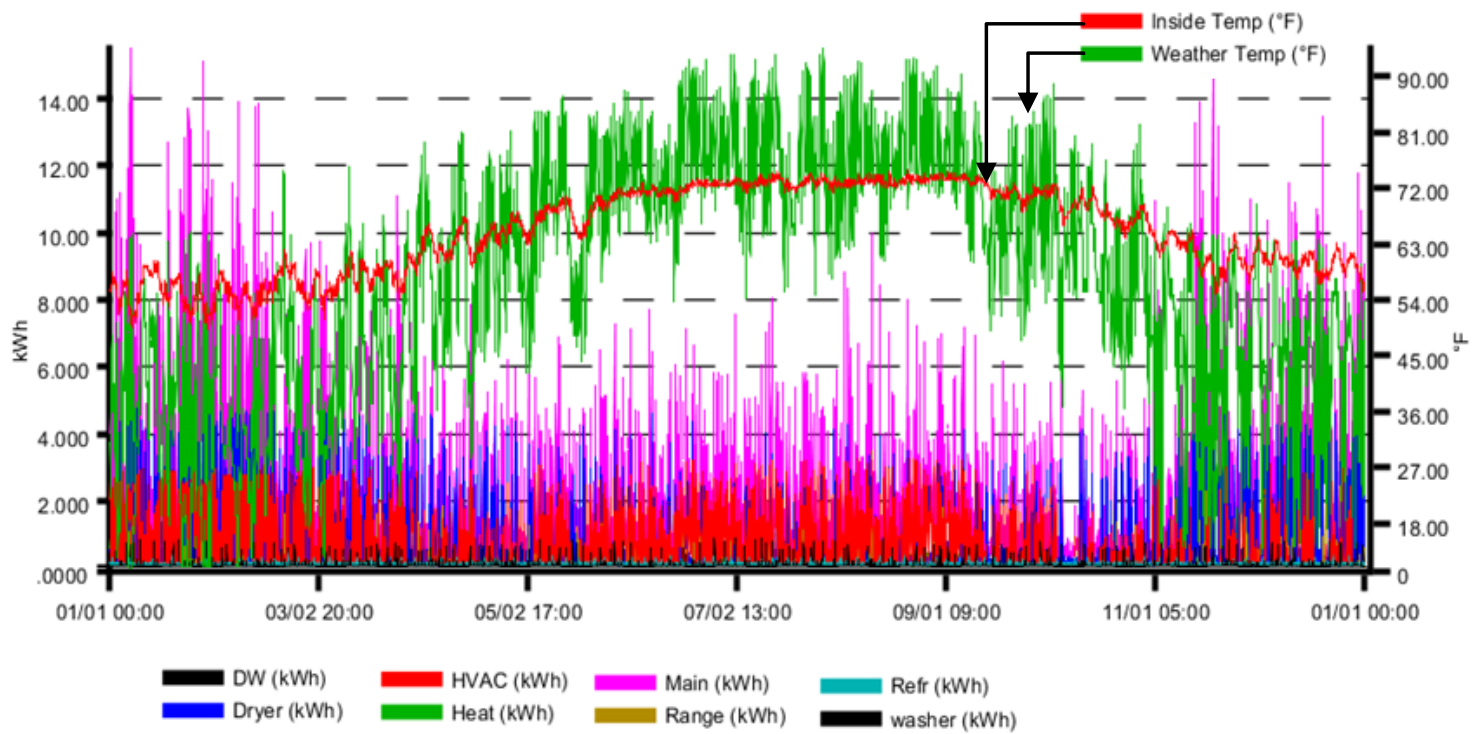

Figure 30: Sub-metered appliances and temperature data for HOME 01 for 2014 
After identifying all significant regression coefficients, we fit appropriate distributions for them using ExpertFit. The distributions for $\lambda$ and $\gamma$ for heating cycle were identified as Uniform $(0.0006,0.0302)$ and Uniform $(0.0156,0.3897)$. Uniform distributions were used partly due to the limited data points for heating cycle. The histogram plots for $\lambda$ and $\gamma$ for heating cycle are shown in Figure 31. Similarly for cooling cycle, the distributions for $\lambda$ and $\gamma$ were identified as $\operatorname{Gamma}(1.028,0.031)$ and Pert $(-1.294,0.038,0.038)$. Figure 32 shows the histograms and probability density functions of the identified distributions. The distributions of $\lambda^{\prime}$ were identified as $P \operatorname{Pert}(0.9976,1.0034,1.0068)$ and $\operatorname{Pert}(0.8579,0.9965,1.0147)$ for the heating and cooling cycles, respectively. The pert distribution is used in place of a triangular distribution, such that a smooth probability density function may be achieved with just the minimum, most likely and maximum values.
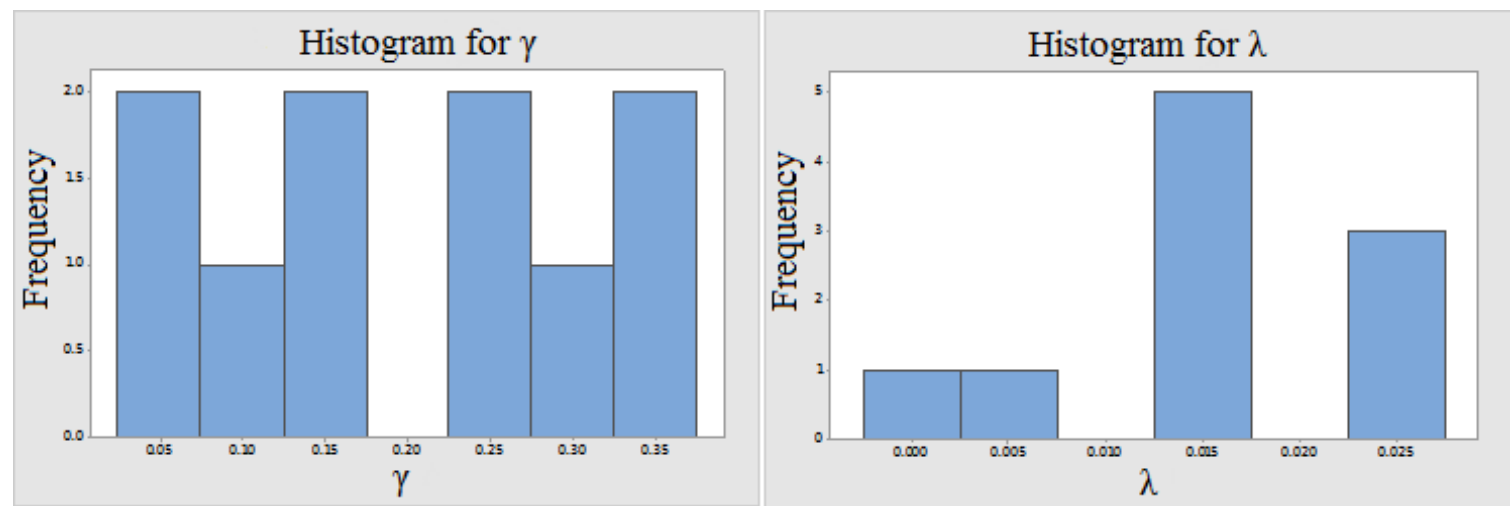

Figure 31: Histogram plots for $\boldsymbol{\lambda}$ and $\boldsymbol{\gamma}$ estimation for heating cycle 

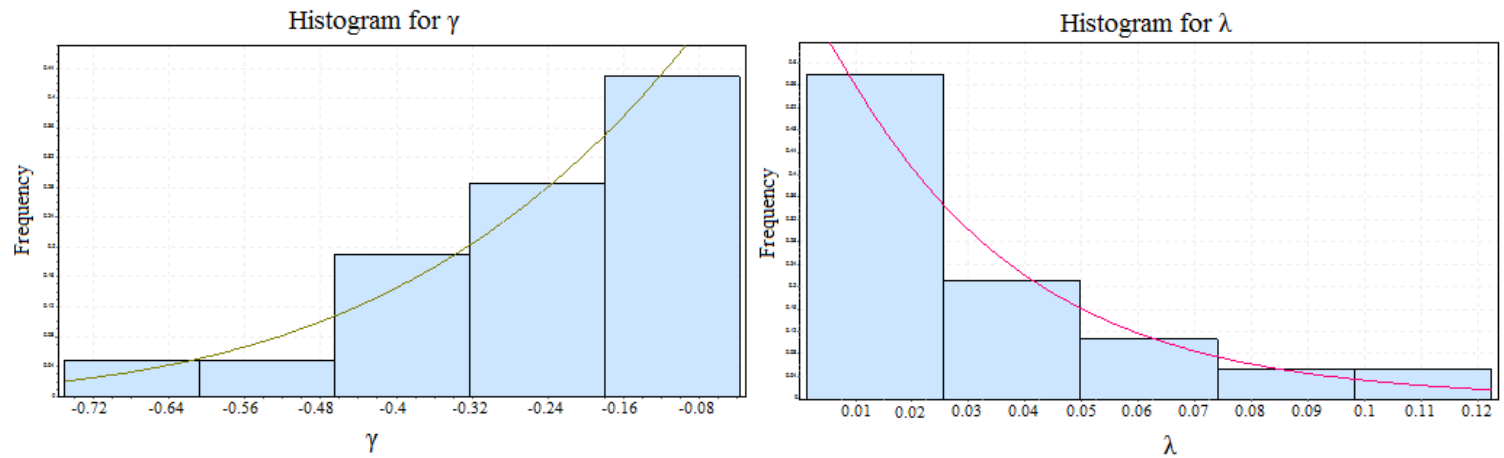

Figure 32: Histogram plots for $\lambda$ and $\gamma$ estimation for cooling cycle

The absolute preferred temperature values were estimated as discussed in Section 3.2.3.1 for each of the 26 test houses. The absolute preferred temperature values were fit to a normal distribution with mean $73.54^{\circ} \mathrm{F}$ and standard deviation $2.028^{\circ} \mathrm{F}$. In order to find the maximum allowed deviation from preferred temperature $D_{t}$ for each house, we studied the fluctuation of inside temperature. The hourly inside temperature for each house was fit to individual normal distributions, the standard deviation of each being assumed as the metric that defines $D_{t}$, since it is after all the deviation from the average. After collecting this value for all the homes, we find that it follows a normal distribution with mean of $3^{\circ} \mathrm{F}$ and standard deviation of $1.5^{\circ} \mathrm{F}$. In the simulation model, each entity is assigned a random value generated from these distributions in a parameter assignment step.

\subsection{MULTI PERIOD OPTIMIZATION}

We propose the use of MPC mechanism to model consumer behavior rather than an active thermostat controller. The MPC mechanism can be applied very well for behavior modeling, considering that the decision maker (consumer) makes consumption 
decisions based on predictions of future system states. Short term forecast of ambient temperature and electricity price is used to evaluate control actions to maximize expected utility during that prediction horizon. However, after implementing the optimal control action at time $t$, the state of the system might have deviated from the projected outcome and thus repeating this process again for the next time period. Based on updated information of the system state, a continuous control is implemented by the decision maker. Compared to a myopic single period optimization, a multi-period optimization is expected to give a better representation of long term consideration by the consumer.

As shown in Figure 29, the optimization step is the central process for the consumer"s decision. Forecast of ambient temperature and electricity pricing is made for a desired number of future timeslots $k$. The optimal sequence of HVAC control actions (command signals) $\vec{w}$ is evaluated for $i=[t, t+k \delta]$ by solving a multi-period optimization problem. The input variables of this problem are current room temperature $T_{t}$, forecast of ambient temperature $\overrightarrow{T^{0}}$ and forecast of prices $\vec{p}$. The output is the optimal sequence of HVAC control actions $\vec{w}$. Recall that in the MPC thermal model, the HVAC command signal $w$ is the input variable that determines the dynamics of the HVAC unit. Particularly, when the unit is turned off $w=0$, and when it is turned on and consumes $(100 w) \%$ of the full power rating $q$ during the time interval, $w$ assumes a value between zero and one. A heuristic based bisection search method is used to optimize the expected utility by starting at a candidate signal. This is discussed in detail in the following sub-sections. We have used the following notations, as shown in Table 8, in our model. 
Table 8: Table of notations used in the optimization problem

\begin{tabular}{|c|l|c|l|}
\hline$\delta$ & Length of time interval & $X_{t}$ & $p_{t} w_{t}=$ Cost of drawing $w_{t}$ load \\
\hline$k$ & Number of intervals for optimization & $Y_{t}$ & $\left|T^{\prime}{ }_{p}-T_{t}\right|=$ Deviation from preferred temp \\
\hline$\Delta$ & {$[t, t+\delta k]=$ Prediction interval } & $U(x)$ & Utility function of an attribute $x$ \\
\hline$w_{t}$ & $\begin{array}{l}\text { HVAC control signal at time } t ; \\
0 \leq w_{t} \leq 1\end{array}$ & $k_{1}, k_{2}$ & Scaling constants for MAUF \\
\hline$T_{t}$ & Room temperature at time $t$ & $\lambda^{\prime}, \lambda, \gamma$ & Thermal parameters of the house \\
\hline$T_{t}^{o}$ & Outside temperature at time $t$ & $q$ & Maximum power rating of HVAC unit \\
\hline$T_{p}$ & Absolute preferred temperature & $p_{t}$ & Price of electricity at time $t$ \\
\hline
\end{tabular}

We divide the day into discrete intervals with length $\delta$, which are reasonable enough for a consumer to make a decision at each interval. In our simulation model $\delta$ is one hour. The MPC process optimizes the system for $k$ intervals, thus the prediction interval is given by $\Delta=[t, t+k \delta]$. The independent variables are ambient temperature and the price of electricity, the predictions of which are assumed to be available to the consumer via smart meters. The dependent variable is the HVAC control signal, which is adjusted via the thermostat set-point. We are modeling the behavior of the consumer and it is desired to minimize energy usage or cost as well as the temperature deviation from a preferred level. This objective is captured by the maximization of total expected utility. The system dynamics of the thermal space is given by Equation (36), as discussed earlier.

We use Equation (37) as the objective function in our multi-period optimization step of the MPC mechanism. Given an absolute preferred temperature of $T_{p}$, and the forecasted values of electricity prices $\vec{p}=\left\{p_{i}\right\}$ and ambient temperature $\overrightarrow{T^{o}}=\left\{T_{i}^{o}\right\}$ 
during the prediction interval $\Delta=[t, t+k \delta]$, we write the control optimization problem (37) - (42) below to be solved by a consumer.

$$
\max _{w_{i}} U=\sum_{i \text { in } \Delta}\left(k_{1} U_{1}\left(X_{i}\right)+k_{2} U_{2}\left(Y_{i}\right)\right)
$$

Subject to:

$$
\begin{array}{ll}
U_{1}\left(X_{i}\right)=f\left(w_{i} p_{i}\right) & i \in \Delta \\
U_{2}\left(Y_{i}\right)=f\left(\left|T^{\prime}{ }_{p}-T_{i}\right|\right) & i \in \Delta \\
T_{p}^{\prime}=\theta T_{i}^{o}+(1-\theta) T_{p} & i \in \Delta \\
T_{i}=\lambda^{\prime} T_{i-1}+\lambda\left(T_{i}^{o}-T_{i-1}\right)+\gamma w_{i} q & i \in \Delta \\
0 \leq w_{i} \leq 1 & \forall i
\end{array}
$$

Constraints (38) and (39) define the individual attribute utility functions for $X$ and $Y$, as described by Equations (29) - (34) in Section 4.1.2. Note that variable $Y$ is defined by the deviation of current room temperature from the actual preferred temperature $\left|T^{\prime}{ }_{p}-T_{t}\right|$. Constraint (40) assures the use of actual preferred value rather than absolute preferred temperature $T_{p}$, which is discussed in Section 3.2.3.1. The thermal dynamics of the house is captured by constraint (41). The normalized value of $w_{i}$ is assured by constraint (42). The values of parameters $\lambda^{\prime}, \lambda$ and $\gamma$ define the thermal characteristics of the house and are randomly generated for each house from previously identified distributions. The thermal behavior of the consumer is also defined by the parameters $T_{p}$ and $\theta$, denoting the absolute preferred temperature for the consumer and the scaling factor that affects his/her actual preferred temperature based on weather 
condition. These parameters are also constant for each household, generated from predefined distributions. Finally the scaling constants of the MAUF $\left(k_{1}, k_{2}\right)$ are defined from a Johnson SB distribution based on the classification of each household.

It is natural to reflect the HVAC control actions in terms of the thermostat setpoint of the house. To achieve the desired control value of $w_{t}$, we imagine that the user either turns the HVAC unit on or off. However, even if the unit is in the auto position with a temperature set-point, the value of $w_{t}$ should logically follow the set-point. For a cooling cycle, we add constraints $(43-46)$ to the original problem to ensure that the HVAC signal only appears $\left(w_{t}>0\right)$ when the thermostat set-point is less than the room temperature. Similar constraints can be added for heating cycle by simply changing the signs in Equations (43) and (44). Although these additional constraints are not necessary for the optimization of $\vec{w}$, we use it as a safe check for the thermostat set-point control.

$$
\begin{array}{lc}
\left(T_{i-1}-T_{i-1}^{s}\right) \leq M y_{i} & i \in \Delta \\
\left(T_{i-1}^{s}-T_{i-1}\right) \leq M\left(1-y_{i}\right) & i \in \Delta \\
w_{i} \leq y_{i} & i \in \Delta \\
y_{i} \in\{0,1\} & \forall i
\end{array}
$$

Under the principal of MPC, the control optimization problem (37) - (42) will be solved for the prediction period $\Delta=[t, t+k \delta]$, and the optimal control signal at time $t$, i.e., the first interval of the prediction period, is used to update the system. Depending on the actual value of price and outside temperature at time $t$, the system may or may not follow the projected path. This process is repeated again in the next decision interval 
$\Delta+\delta=[t+\delta, t+(k+1) \delta]$. Note that although the actual response of the system may differ slightly from the projected response, mainly due to inaccuracy in forecasting the independent variables, the continuous optimization of the MPC mechanism allows the system to converge to the reference output better than a single period optimization.

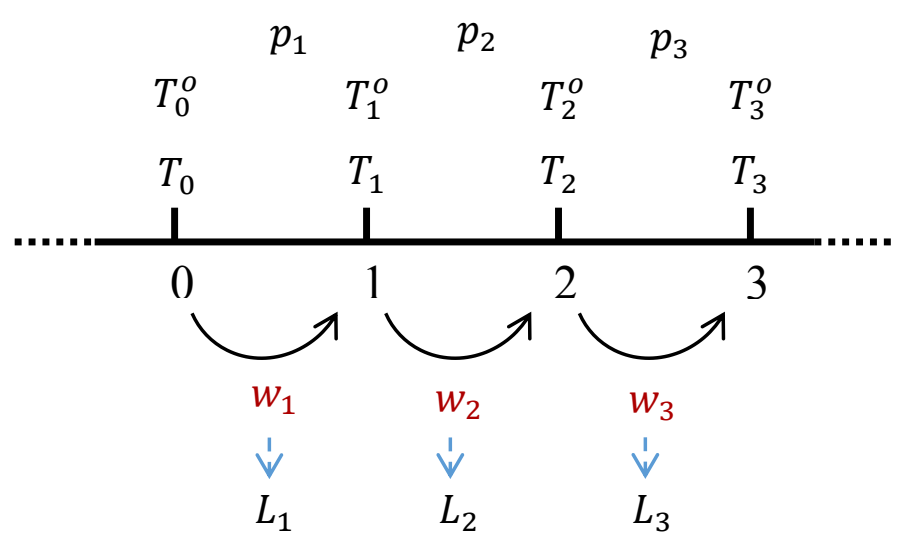

Figure 33: Three period graphical representation of the optimization problem

We consider a three period prediction period in our simulation model. Figure 33 shows a graphical representation of the different variables for the optimization period. Let $T_{0}$ and $T_{0}^{o}$ be the current room temperature and outside temperature, which are obviously known. The decision variable for the optimization problem is the sequence of HVAC control signals $\vec{w}=\left\{w_{1}, w_{2}, w_{3}\right\}$. Let the predicted variables for electricity price and outside temperature for the timeslots $i=\{1,2,3\}$ be given by $\vec{p}=\left\{p_{1}, p_{2}, p_{3}\right\}$ and $\overrightarrow{T^{o}}=\left\{T_{1}^{o}, T_{2}^{o}, T_{3}^{o}\right\}$ respectively. Each time period has a load consumption, denoted by $\left\{L_{1}, L_{2}, L_{3}\right\}$, corresponding to the respective HVAC control signal plus the base load for that time period. 
The cost of energy usage is evaluated as $X=L_{i} p_{i}$ in the general form. However, when a demand charge is introduced we separate the cost into energy and demand charge rates, denoted by $p_{e}$ and $p_{d}$. The estimation of the cost due to energy charge is straight forward and applied to the average load during the three periods (Equation 47). The demand charge is applied only once to the peak load of the month. Different pricing methods may apply it to the user peak consumption or the coincident peak load. The coincident peak load is the amount of load consumed by a user during the hour of the month determined as the system peak. In either case we need to track the incumbent peak load for this purpose. Let $L_{m}$ denote the incumbent peak load that was observed during the current month. If the demand charge is applied to the user peak, then Equation (48) is used to determine the demand charge. If the demand charge is applied to coincident peak, then it is of high importance to the user to whether or not any of the timeslots in the prediction period reaches system peak. If $\emptyset_{i}$ is used to denote the probability that period $i$ will reach system peak, then Equation (49) can be used to determine the average cost of demand charge for the prediction period. The method used to estimate $\emptyset_{i}$ is discussed in Section 4.4

$$
\begin{aligned}
& X_{e}=\left(p_{e 1} L_{1}+p_{e 2} L_{2}+p_{e 3} L_{3}\right) / 3 \\
& X_{d}=p_{d} * \max \left\{L_{1}, L_{2}, L_{3}, L_{m}\right\} \\
& X_{d}=p_{d}\left(\sum_{i=1}^{3}\left\{\emptyset_{i} L_{i}+\left(1-\emptyset_{i}\right) L_{m}\right\}\right) / 3
\end{aligned}
$$

The average discomfort value is dependent on the room temperature for the respective periods denoted by $\left\{T_{1}, T_{2}, T_{3}\right\}$ in Figure 33. The room temperature $T_{i}$ is a 
function of $T_{i-1}, w_{i}$ and $T_{i}^{o}$. The average discomfort value is then given by Equation (50), where $T^{\prime}{ }_{p}$ is the actual preferred temperature of the household.

$$
Y=\left(\sum_{i=1}^{3}\left|T_{p}^{\prime}-T_{i}\right|\right) / 3
$$

\subsubsection{Bisection Search}

The above optimization problem is a multi-period nonlinear program. Because solving the above nonlinear optimization model at each iteration of the MPC process may require excessive computational time, and there are usually many iterations involved depending the length of unit time interval $\delta$, it is efficient to solve (37)-(46) repeatedly using a heuristic method. Wilson and Dowlatabadi (2007) have also noted that heuristics are often adopted more than exact methods by decision makers in practice due to reduced cognitive and computational requirements. In particular, they propose a recognition heuristic that favors familiar solutions, which matches energy users ${ }^{\text {ee }}$ pervious behavior. This is well supported by the theory of bounded rationality in behavioral economics literature. The concepts of human behavior, rationality and decision making were proposed by Herbert Simon in a career long effort to explain rational behavior. Many researchers in behavioral economics have built upon the work of Simon to support the theory of bounded rationality.

The idea behind bounded rationality is that in a decision making situation, the rational behavior of consumers is limited by the information at hand, cognitive limitations of their mind and the limited amount of time they are willing to spend making that decision. If a consumer is faced with a large number of options they usually tend to 
make sub-optimal choices, as compared to just a few options. That is why it is probably easier to select the best phone carrier (out of maybe 5 options) than to choose the best breakfast cereal (out of more than 50 varieties). In our context, we assume that a consumer is faced with the decision of energy usage based on forecasts of temperature and prices. Since this decision is to be made at every hour, the consumer does not necessarily choose the global optimal solution. Hence we propose the simplification of the optimization problem using a heuristic based method including a recognition heuristic and a bi-section search heuristic with a discretely approximated search space.

In addition to the recognition heuristic, a bi-section search method is employed to find an approximate solution to (37)-(46) at each iteration of the MPC process. Algorithm 2 below outlines the heuristic method for solving the optimal HVAC control problem for time $[t, t+2]$. First, in Steps 0 and 1 , the algorithm recognizes familiar set-point values for any given particular times. In Step 2, depending on whether the temperature is below or above this set-point, the algorithm sets $w_{t}=0$ or 1 based on cooling or heating cycles. Then in Step 3, it solves for the room temperature of the subsequent interval based on the thermal dynamics of the system. Subsequently, in Steps 4 and 5, the algorithm uses bi-section search technique to decide whether to increase or decrease $w_{t}$. The finite difference method is a numerical method to approximate differential equations to numerically find the gradient. The derivative $U^{\prime}\left(w_{t}\right)$ is estimated by solving for $U\left(w_{t}+\Delta w_{t}\right)$ at a very small step $\Delta w_{t}$. Finally, the heuristic terminates if the improvement in the objective value is less than a predetermined threshold $\varepsilon$. 
Algorithm 2. A Bi-section Search Heuristic for Optimal HVAC Control

Step 0: Initialize forecast price and temperature data for all $[t, t+2]$

Step 1: Find the familiar set point, $T_{t}^{s}$, for the current time on previous day

Step 2: If $T_{t}^{s}<T_{t}$, then set $w_{t}=1$ for cooling cycle or $w_{t}=0$ for heating cycle; else $w_{t}=0$ for cooling cycle or $w_{t}=1$ for heating cycle

Step 3: Calculate $T_{t+1}$ using equation (41) and evaluate $U$

Step 4: Estimate $U^{\prime}\left(w_{t}\right)$ using finite difference method

Step 5: Use bisection search method to find next candidate $w_{t}$, based on $U^{\prime}\left(w_{t}\right)<$ or $>0$

Step 6: Calculate new $T_{t+1}$ and $U$ similar to Step 3

Step 8: Stop if $U^{\prime}\left(w_{t}\right)=0$ or $\left|U_{i}-U_{i-1}\right| \leq \varepsilon$; Else Go to Step 4

Since the optimization problem (37)-(46) is a multi-period optimization, the solution to $w_{t}$ alone is not enough. We are interested in finding the optimal sequence $\left\{w_{t}, w_{t+1}, w_{t+2}\right\}$. For every candidate point in $w_{t}$, there exists an optimal sequence of $\left\{w_{t+1}, w_{t+2}\right\}$ that produces the maximum utility value $U$ is Step 3 of Algorithm 2. Instead of trying to find the global optimal solution in every subsequent timeslot, we approximate the continuous solution space to a manageable discrete space. We define the solution space for $w_{t+1} \in\{0,0.25,0.5,0.75,1\}$ and $w_{t+2} \in\{0,0.25,0.5,0.75,1\}$. This reduces the total solution space to only 25 points for every candidate point in $w_{t}$, thus allowing a complete enumeration to evaluate the optimal value of $U$ in Step 3.

\subsection{PEAK FORECASTING WITH CONDITIONAL MARKOV CHAIN}

Dynamic variable pricing can have a very significant impact in DSM with the development of smart grid networks. Existing DR programs use a variety of pricing mechanisms to influence consumer behavior but are mostly limited to static variable 
pricings such as time of use (TOU) pricing, variable unit charge contracts, variable block charges, variable peak pricing (VPP), peak rebates, etc. The use of dynamic variable pricing methods such as real time pricing (RTP), critical peak pricing (CPP), direct load control (DLC), etc. are used by very few utility companies at the moment. However, with the ability to communicate load data and information with consumers through the smart grid framework, the role of dynamic variable pricing is expected to increase.

The use of demand charge is not new in electricity distribution. Industrial customers are usually billed with a demand charge applied to their peak consumption in addition to the regular energy usage. In recent years residential customers have also been offered pricing models that use demand charge in addition to TOU or fixed rates. The potential for designing a dynamic pricing model using demand charge is promising. We design a simple rate system for residential consumers as shown in Equation (51). The monthly bill includes a basic charge $p_{f}$, an energy charge $p_{e}$ applied to the total $\mathrm{kWh}$ used during the month, and a demand charge $p_{d}$ applied to the coincident load $L_{m}(\mathrm{~kW})$ consumed during the system peak hour.

$$
\text { Cost of Electricity }=p_{f}+p_{e} \sum_{t} L_{t}+p_{d} L_{m}
$$

Please note that demand charge in itself is a non-volumetric rate, as the cost attributed to the demand charge is evaluated only on the $\mathrm{kW}$ amount during a single peak hour. In contrast, other rate structures are volumetric in nature, where the rate is applied to the total $\mathrm{kWh}$ consumed in the entire billing period. The consumer is not charged for their individual peak power consumption, but the coincident power during the system 
wide peak of the month. The individual peak is irrelevant to the utility company with the goal of reducing overall system peak. Individual peaks are only of interest if they are contributing to system peak. The uncertainty of when the system will peak during a month makes this dynamic in nature. The utility company can provide the information of current system load and incumbent system peak to their consumers via various communication portals. With the development of smart grid and smart meters, this information could be sent directly to the consumer through their smart meters. Glasgow Electric Plant Board (GEPB) for example provides system load information to their consumers through their website as shown in Figure 34. The consumer is thus faced with the task of avoiding high load consumption when the system reaches a potential peak, i.e. when current load level crosses the incumbent peak level for the month, thus creating a new incumbent peak.

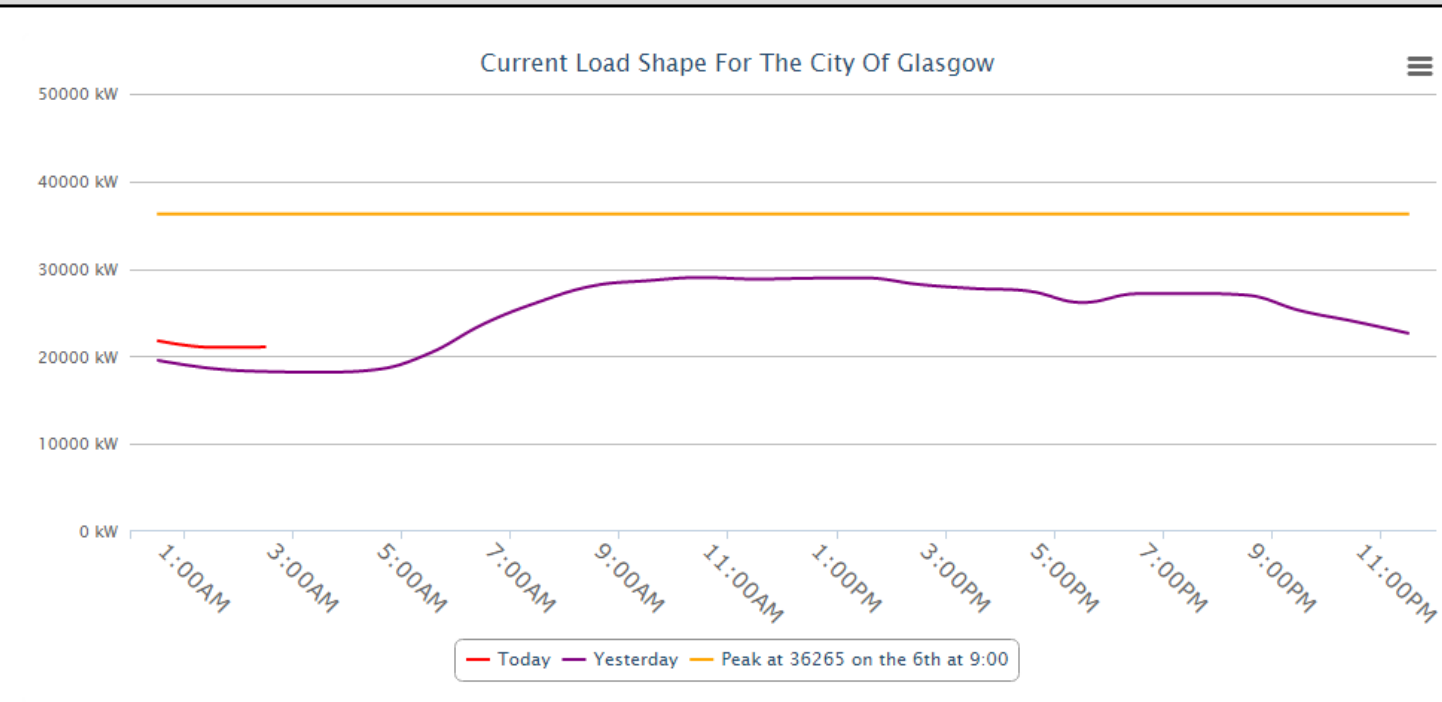

Did you know you can help keep Glasgow's electric rates low? This graph shows the daily usage of the EPB's customers. The purple line shows yesterday's usage and the red line shows today's. The orange line represents the peak usage for the month, and what TVA uses to calculate our monthly payments. To help keep our rates low, please reduce your power usage when you see the red line approaching the orange line.

Figure 34: Screenshot of system load information provided by GEPB on their website 
There exists a probability that the system state will reach a new peak given current system conditions. As explained in earlier sections, we use this probability to estimate demand charges in order to evaluate the utility of the consumer. We propose the use of a conditional Markov chain to model this probability. First of all we define the different states of the system. Let the current system load or power level be given by $P$ and the current incumbent peak for the month be given by $\tilde{P}$. Based on the proximity of $P$ to $\tilde{P}$, we define the various states $S \in\left\{S_{0}, S_{1}, S_{2}\right\}$ as follows, indicating the peak, intermediate and off-peak states. Here, $k$ is a predefined desired power interval (e.g., $20 \%$ of $\tilde{P}$ ) for the markov chain. Figure 35 illustrates the various states.

- Peak state: $S_{0} \ddot{=}(\tilde{P}-P)=0$

- Intermediate state: $S_{1} \ddot{=} 0<(\tilde{P}-P) \leq k$

- Off-peak state: $S_{2} \ddot{=}(\tilde{P}-P)>k$

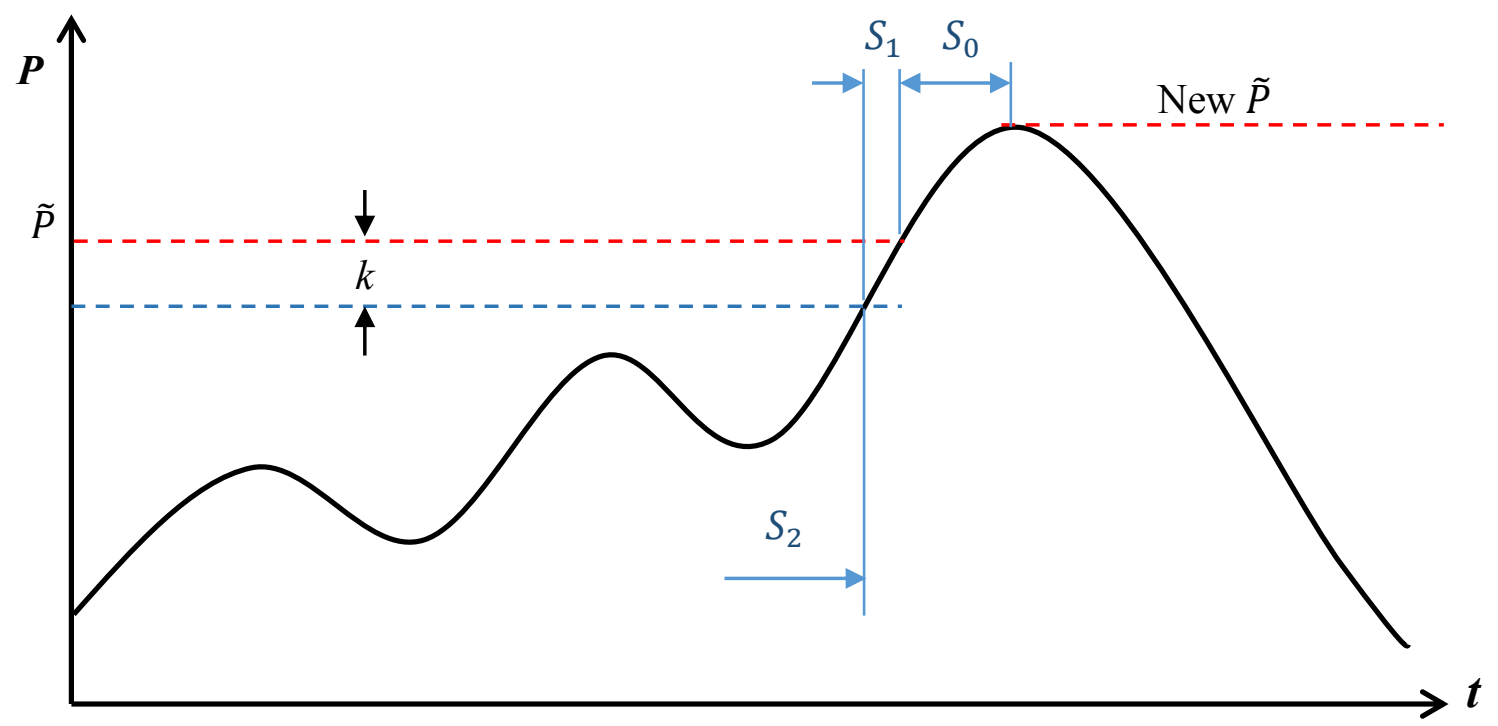

Figure 35: Illustration of various states for the Markov chain model 
A transition probability matrix $R$ is used to determine the likelihood of the system to go from one state to another. Equation (52) shows the transition matrix for our model where $p_{i j}$ is the probability that the system goes from state $S_{i}$ to $S_{j}$. Data regarding the state transitions are recorded continuously and used to update the transition matrix. The transition probability matrix evolves continuously over time as new data is available of the state transitions. However, at a particular time, the transition matrix $R$ is assumed to be time-homogeneous and the probability of reaching a certain state in $m$ number of steps (or time periods) is simply evaluated using $R^{m}$.

$$
R=\left(\begin{array}{lll}
p_{00} & p_{01} & p_{02} \\
p_{10} & p_{11} & p_{12} \\
p_{20} & p_{21} & p_{22}
\end{array}\right)
$$

The load consumption behavior of residential consumers in affected greatly by the weather condition as well. The probability of a certain state transition may be different under varying weather conditions. For example, if the outside temperature is very high and the system is currently at an intermediate state (close to peak), there is a higher probability of the system going to peak state as compared to a situation where outside temperature is milder. We thus condition the Markov chain on weather condition, which is divided into four regions based on ambient temperature as follows.

- Low load: $T^{o}<70^{\circ} \mathrm{F}$ for summer, $T^{o}>50^{\circ} \mathrm{F}$ for winter

- Medium load: $70^{\circ} \mathrm{F} \leq T^{o}<80^{\circ} \mathrm{F}$ for summer, $50^{\circ} \mathrm{F} \geq T^{o}>40^{\circ} \mathrm{F}$ for winter

- High load: $80^{\circ} \mathrm{F} \leq T^{o}<90^{\circ} \mathrm{F}$ for summer, $40^{\circ} \mathrm{F} \geq T^{o}>30^{\circ} \mathrm{F}$ for winter

- Extreme load: $T^{o} \geq 90^{\circ} \mathrm{F}$ for summer, $T^{o} \leq 30^{\circ} \mathrm{F}$ for winter 
Based on the appropriate weather condition, the consumer estimates the probability that a future timeslot will go to peak state from the corresponding transition matrix $\left\{R_{l}, R_{m}, R_{h}, R_{x}\right\}$. As mentioned earlier, we assume that the Markov chain is timehomogenous at any time instance for prediction purpose. We can also use the ergodic property of the Markov chain to estimate the steady state probability of going to peak state.

Definition: A Markov chain is said to be ergodic if any state can be reached from any other state in $n$ steps such that $n \geq 1$.

If the steady state probabilities are represented by the vector $\pi=\left(\pi_{0}, \pi_{1}, \pi_{2}\right)$, where $\pi_{j}$ is the steady state probability of state $j$, then we have the following equations for an ergodic Markov chain with transition matrix $R$.

$$
\begin{aligned}
& \pi^{T}=\pi^{T} R \\
& \sum_{j} \pi_{j}=1
\end{aligned}
$$




\section{CHAPTER V}

\section{SIMULATION RESULTS}

The purpose of the simulation model described in the previous section is to accurately represent the load consumption behavior of a population in response to various pricing mechanisms, such that novel pricing structures may be studied in a computer environment before applying it in the real world. The trade-off between cost and convenience/comfort for each unique household is accounted by their utility functions thus allowing for a rich model that can predict system level changes caused by DR programs. We analyze the effects of various pricing structures on the behavior of different types of consumers using simulation experiments. Based on our proposition that people are affected not only by cost but also the comfort of their energy usage, we expect to see varying responses from households with different utility functions and preferences. The randomization of household characteristics by assigning distributed parameters (risk level, thermal parameters, utility coefficients, etc.) has been discussed in earlier sections. We initially use a population size of $n=30$ which we increase to $n=100$ for the TCL model. The determination of the scaling constants for the multiattribute utility functions is ideally done by interviewing households about their preferences over various conditions. Households of different categories will have varying preferences and thus different scaling constants. Since such type of data is not available 
for a large enough sample, we assume these values from a Johnson SB distribution as discussed in Section 3.2.4.

We use two main parameters to evaluate the load profiles in our study. First, the load factor (LF) is calculated as the ratio of average load over peak load in a daily cycle (Equation 55). This ratio helps us to measure the reduction in the peak load, since the average load is expected to remain same for a particular population based on the load demand. A low LF indicates a load profile with high peaks, whereas a higher LF indicates a relatively flat profile. A second parameter is also used to measure load variation (VAR), which is calculated as the sum of the squared deviation from average as shown in Equation (56). The total number of time intervals $t$ in a day is used as 48 for NTL model (half hour intervals) and 24 for TCL model (hour intervals). Note that instead of the actual value of deviation from the average, we use the ratio of the deviation over the average load as the error term in order to normalize the parameter.

$$
\begin{gathered}
L F=\frac{\text { AvgLoad }}{\text { PeakLoad }} \\
V A R=\sum_{i=1}^{t}\left(\frac{\text { Load }_{i}-\text { AvgLoad }}{\text { AvgLoad }}\right)^{2}
\end{gathered}
$$

The principles of simulation rely on randomness of the process being studied and the analysis of the desired output as an estimate rather than a definitive solution. In other words, the primary reason for the use of simulation is the existence of uncertainty in a system that cannot be defined by deterministic mathematical equations, while simulation is able to use the randomization of such processes to estimate a statistically significant response. In our simulation model, the randomness is brought about by various factors 
such as utility functions with varying risk natures, preferred temperature of households, preferred schedule of using NTL appliances, energy rating of each appliance, energy consumed by each consumer, ambient temperature, etc. Due to these random factors, the output load for each day is expected to vary slightly. Also, for TCL loads, the consumption is a function of ambient temperature which differs from day to day, thus causing fluctuation in daily response, which is expected. We run the NTL model for 10 days and the TCL model for 1-3 months in order to estimate the load response. The warmup period for each model is estimated from a steady state analysis of a selected output variable, e.g. system load response, steady state probability of peak state, real-time electricity rate, etc.

\subsection{MODEL VERIFICATION AND LOAD VALIDATION}

Before we can conduct experiments using the simulation model, we have to be certain that it behaves the way we want it to. The ultimate goal of producing an accurate and credible simulation relies on the verification and validation of the model. First of all the model verification is done to ensure that the implementation of the concept is correct. In our model, we rely on the varying trade-off nature of the consumers as described by their utility functions as the foundation. The amount of load consumed by a household is dependent on the willingness to respond to variable pricing. As described in Section 3 we define the household's trade-off behavior using different scaling constants in their MAUF $\left(k_{1}, k_{2}\right)$. High income households are assumed to be "comfort favoring" with a high weightage for the utility of comfort, whereas low income households are assumed to be "cost favoring" with a high weightage for the utility of cost. The risk natures of the 
homes are defined by the curvature of their utility functions $\left(\alpha_{1}, \alpha_{2}\right)$. We verify that the utility functions of the simulated entities return appropriate values. A test house is assigned with known values of $k_{1}, k_{2}, \alpha_{1}, \alpha_{2}$ and the utility function values of each variable $(X$ and $Y$ ) are recorded to verify the intended characteristic. Four types of test houses were created with different parameters as shown below.

- Cost favoring / Risk Neutral: $k_{1}=0.9, k_{2}=0.1, \alpha_{1}=-1, \alpha_{2}=-0.1$

- Cost favoring / Risk Averse: $k_{1}=0.9, k_{2}=0.1, \alpha_{1}=-8, \alpha_{2}=-0.8$

- Comfort favoring / Risk Neutral: $k_{1}=0.1, k_{2}=0.9, \alpha_{1}=-1, \alpha_{2}=-0.1$

- Comfort favoring / Risk Averse: $k_{1}=0.1, k_{2}=0.9, \alpha_{1}=-8, \alpha_{2}=-0.8$

In order to verify the expected behavior of these test houses as defined by their utility functions, the values of $X$ and $Y$ along with their corresponding utility values, $U_{1}(X)$ and $U_{2}(Y)$, that result in the optimal decision for each hour were recorded. The utility functions and average responses for each of the variables were first compared among the test houses using a standard flat rate model. Figure 36 shows the utility function curves and average values for cost and discomfort for each type of house under the flat rate model. We can clearly identify that the utility functions for risk averse homes have higher curvature with higher marginal utility at higher cost or discomfort, whereas risk neutral homes have an almost linear function. The utility values are also bounded between 0 and 1 . More importantly, we can see that the average cost per hour is about $\$ 0.18$ for cost favoring homes while it is about $\$ 0.24$ for comfort favoring homes. The average discomfort on the other hand is $2.55^{\circ} \mathrm{F}$ for cost favoring homes compared to a much lower $0.6^{\circ} \mathrm{F}$ for comfort favoring homes, as we would expect. 

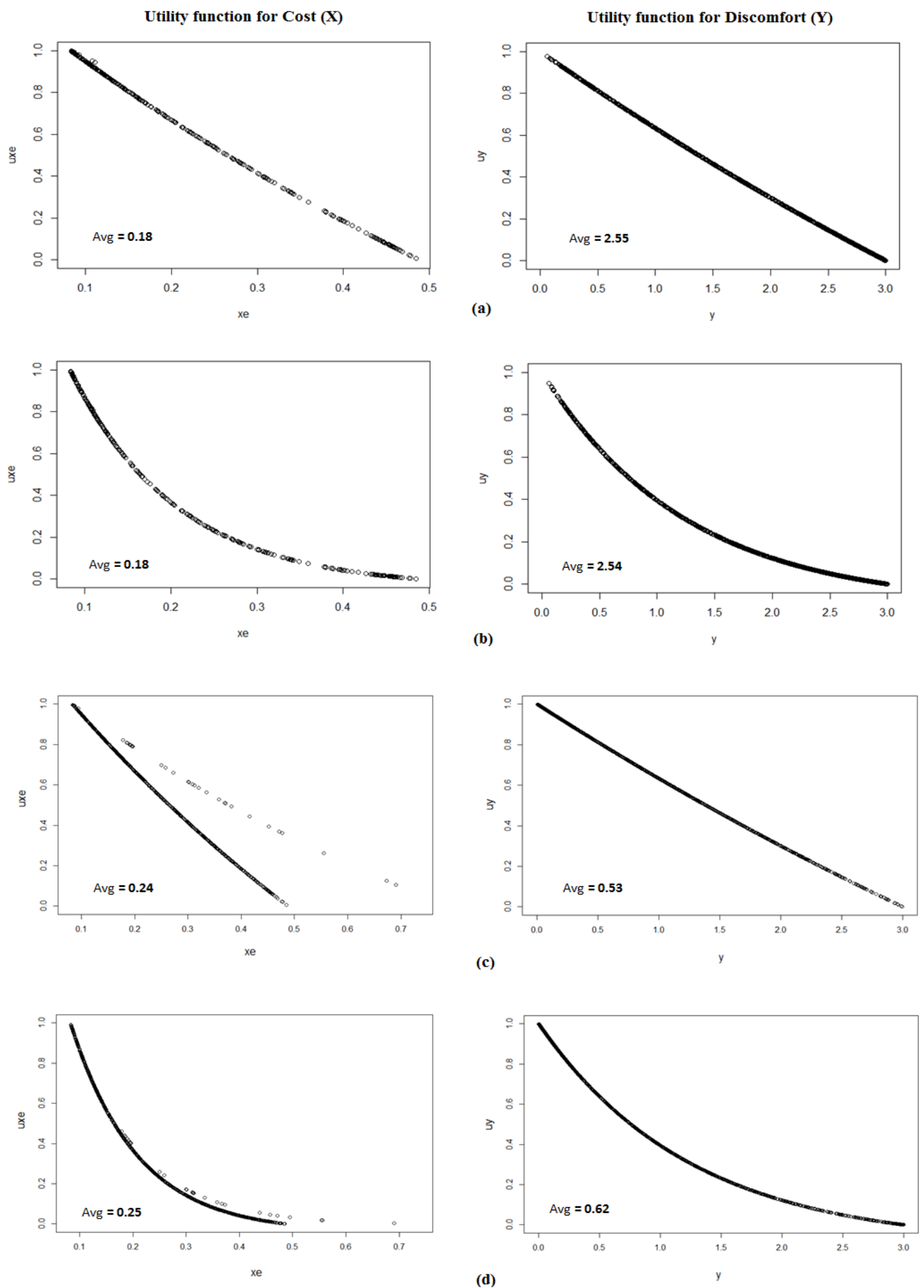

Figure 36: Utility functions and average values of $\mathrm{X}$ and $\mathrm{Y}$ for (a) Cost favoring / Risk neutral, (b) Cost favoring / Risk Averse, (c) Comfort favoring / Risk neutral and (d) Comfort favoring / Risk averse test houses. 
The trade-off behavior of different types of households in the simulation model is verified under a standard flat rate model. We further check the consistency of this behavior by analyzing the utility functions and average cost and discomfort for the test houses under Time-of-Use (TOU) and a system peak demand charge rates. The utility functions and corresponding average cost and discomfort values for each test house under different rate structures are presented in Appendix A. The response behavior of cost favoring and comfort favoring test houses are as expected. We see a higher discomfort and lower average cost for cost favoring homes, while a lower discomfort and a slightly higher average cost for comfort favoring homes.

When comparing the flat rate model to the TOU model, we see that the cost favoring homes have a reduction in average cost while keeping the discomfort at the same level or even slightly higher. This makes sense since a cost-sensitive household is expected to take advantage of the TOU rates to reduce their average costs. The comfort favoring homes on the other hand show contrasting behavior. The average cost is higher under the TOU rates, while keeping discomfort constant or even lower. This also makes sense as a comfort-sensitive household will not be affected by TOU rates to change their behavior and thus result in higher average costs. Based on the pricing design of the system peak demand charge rates, we can see a general reduction in the hourly energy charge $X_{e}$ while a high demand charge $X_{d}$ is assessed. The trade-off behaviors of the cost favoring and comfort favoring houses can also be verified under this rate structure. Both the average energy charge and demand charge are higher for comfort favoring houses while the average discomfort is lower (see Appendix A). 
Recall that the preferred times of usage for the NTL appliances (Washer, Dryer and Dishwasher) were estimated from the prevalent literature of end use household appliance usage patterns (Section 4.1.1). We used a bimodal distribution for the usage times of washer and dryer as $7 \mathrm{AM}-9 \mathrm{AM}(30 \%)$ and $4 \mathrm{PM}-8 \mathrm{PM}(70 \%)$. For the usage of dishwasher, we simply assumed the times as 8 PM - 10 PM. This NTL appliance usage schedules are assigned to the households by generating the times from a uniform distribution between the above times.

As a verification of these assumptions, we looked at the average appliance level consumption data from the 26 sub-metered test homes from Glasgow, KY for the year 2013. The average daily consumption of washer, dryer and dishwasher loads were calculated for each test house. The general assumption of the bimodal distribution for washer and dryer is supported by the data as shown in Figure 37 and Figure 38.

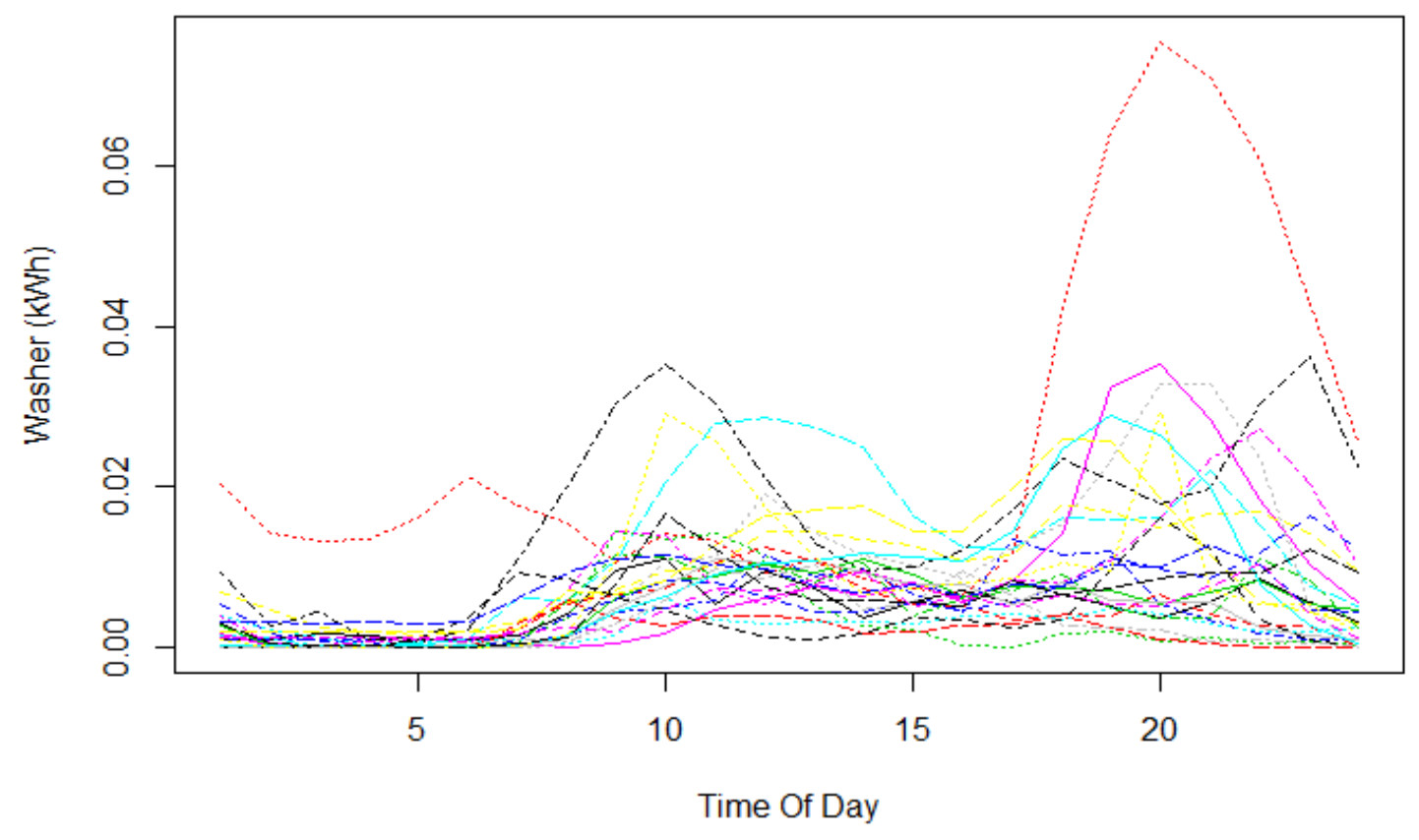

Figure 37: Average daily consumption profiles of washer load by 26 test homes in 2013 
The usage times for washer and dryer are also close to the values that we have assumed in the simulation model. Considering that our assumed usage times indicate the start time for the appliance, we can see a peak in consumption at around 10 AM during the morning period and a peak at around $8 \mathrm{PM}$ at night. This behavior observation can be used to justify the use of the preferred usage times for washer and dryer.

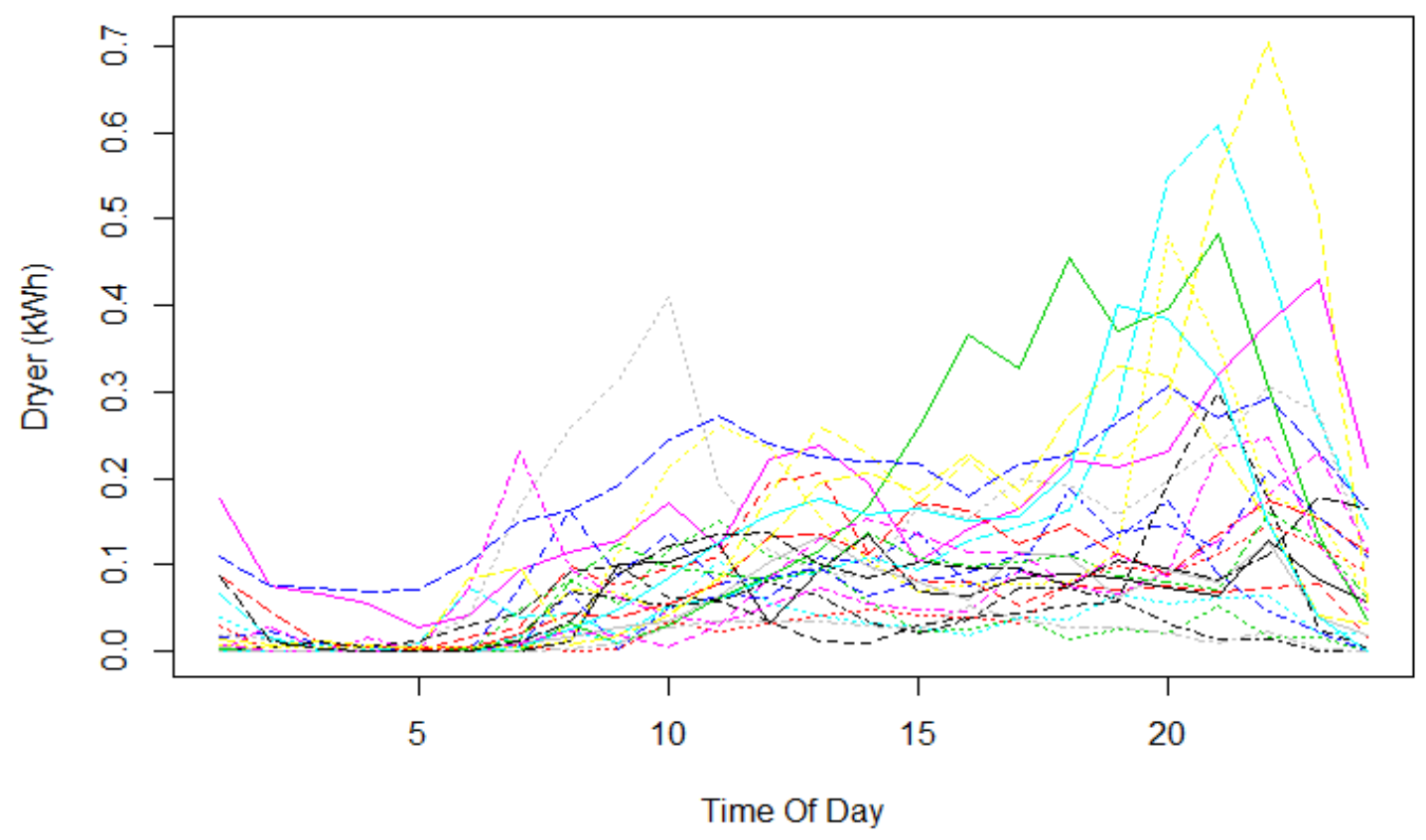

Figure 38: Average daily consumption profiles of dryer load by 26 test homes in 2013

Similarly, the average daily consumption pattern of dishwasher loads by the 26 test homes indicate that most of this load is consumed after $8 \mathrm{PM}$, although we do see a consistent mild consumption during the day time as well (Figure 39). We maintain that our assumption of dishwasher usage schedule of $8 \mathrm{PM}-10 \mathrm{PM}$ is valid for the purpose of our study, and ignore the day-time use of dishwasher. The goal of our model is to study the load shift behavior rather than actual time of consumption. 


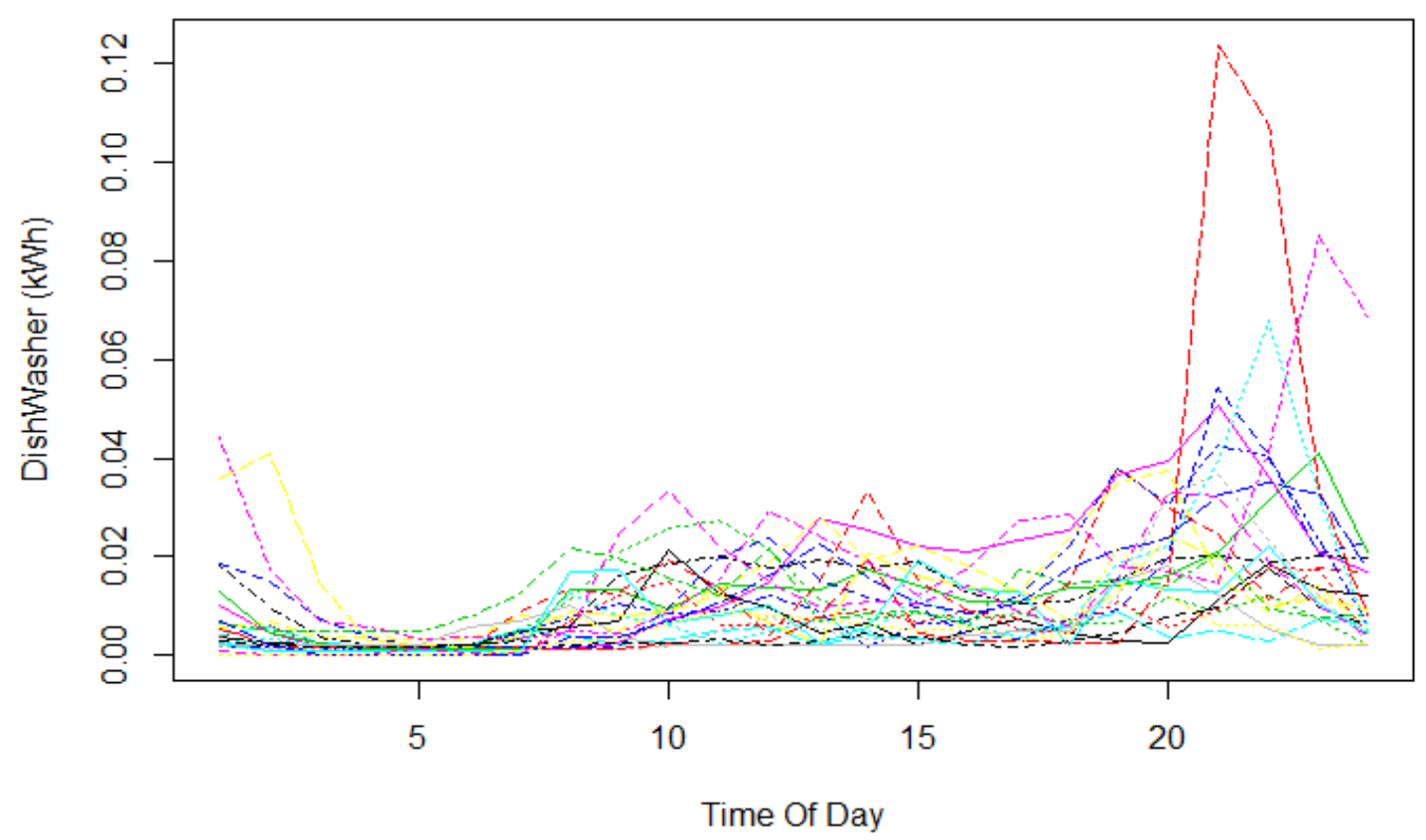

Figure 39: Average daily consumption profiles of dishwasher by 26 test homes in 2013

The simulation of TCL model is primarily based on the consumption of HVAC and is thus highly dependent on the weather data. The total load profile is calculated as the sum of the base load and the variable HVAC load. By populating the simulation model with households of distributed nature, we expect to simulate the system load response as closely following the changes in outside temperature. We use the weather data of 2013 for Glasgow, KY as the input variable in our model. Thus we will use the system load profile of Glasgow, KY obtained from Glasgow Electric Plant Board (GEPB) to validate our model.

We use a population size of $n=100$ and run the simulation model from June to August under a standard flat rate pricing scheme. Since GEPB serves around 5000 - 6000 residential homes, we have to scale up the simulated load profile until the error between the actual and simulated load is minimized. The minimum error was obtained when 
scaling the simulation results to 5500 households, which corresponds to the actual population size. In comparing the actual system load profile to the simulated one, we observe a close match that follows the weather pattern for all days except weekends and holidays. Figure 40 shows the comparison of the simulated system load and actual system load for the first week of June, 2013. We can see that the first two days do not match the actual data but the next five days do. 6/1/2013 and 6/2/2013 happen to be weekends, and we are aware that weekend consumption is much lower than weekday consumption. Since we have not included the effect of weekends in our model we simply ignore this. For the purpose of load validation we remove all weekends and holidays. However, the overall system response will include all 30 days, ignoring weekend effects. The complete load validation graph for weekdays can be seen in Appendix B.

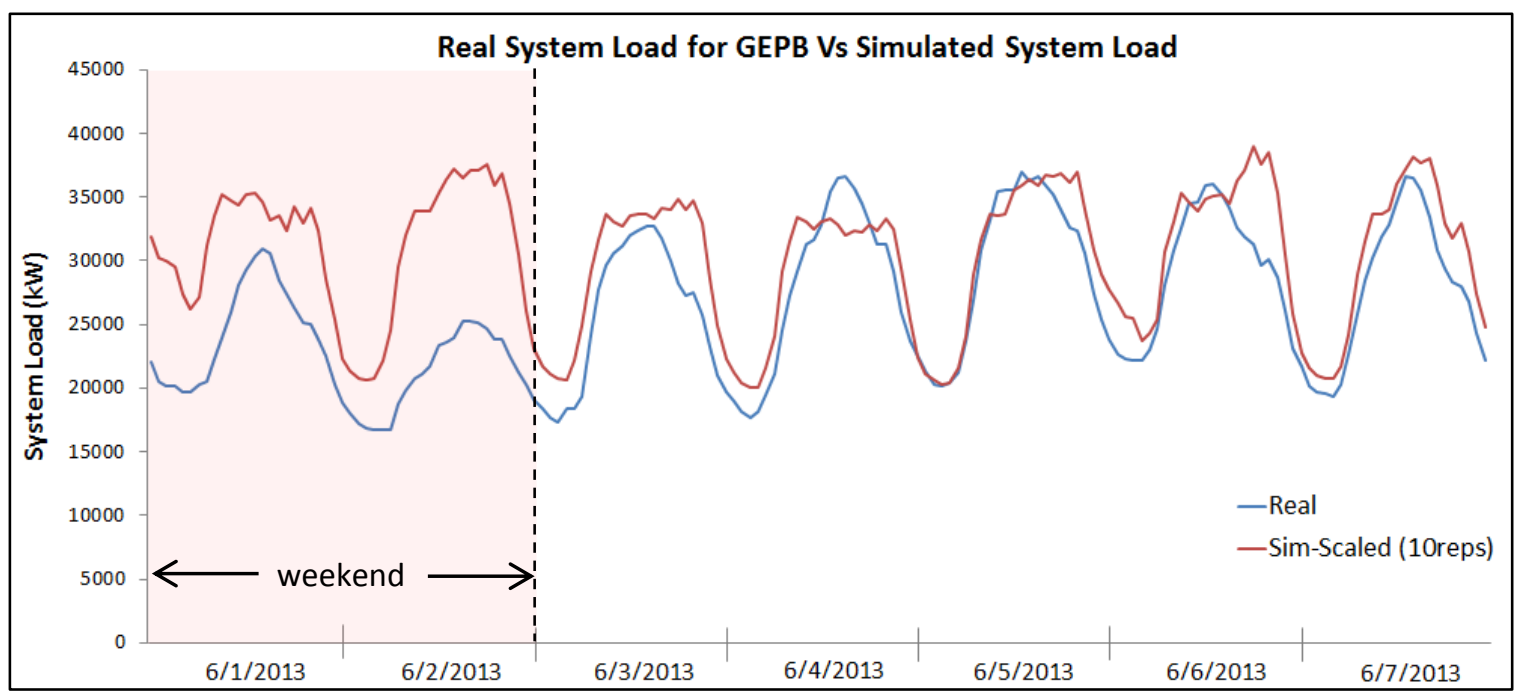

Figure 40: Load validation of TCL model using GEPB system load for 2013

In order to calculate the model error, we first remove all the weekends and holidays. For the remainder of the days we calculate the percentage error for each hour by 
taking the difference of the actual load and the simulated load and dividing it by the actual load. The average percent error is then obtained by taking the mean of these values, which was found to be 0.05382656 . This indicates that the simulated load profile has an average error of about $5.4 \%$ when compared to the actual load.

\subsection{DESIGN OF EXPERIMENT FOR RESIDENTIAL RATES}

The load consumption behavior of a sample population under different pricing schemes can be studied using the simulation models discussed previously in Section 4 . The load consumption behavior of non-thermostatic loads and thermostatic loads are examined separately using the NTL and TCL models due to their variable usage patterns. The convenience factor related to the NTL model only assumes the ability to use an appliance at a desired time, whereas the comfort factor related to the TCL model is evaluated as a function of the thermal behavior of the house. For this reason, we use different pricing models to study load shifting behavior for NTL and TCL models.

Variable pricing mechanisms can be divided into two categories, namely static and dynamic variable pricings. The difference between the two pricing models lies in the uncertainty of electricity rate at any given period. Pricing structures where the rate for a given time period is constant although different time periods may be assigned different rates are considered as static variable pricing. In the context of this dissertation, we use Time-of-Use (TOU) rates and their variants for this type of pricing model. On the other hand the scope for dynamic variable pricing is greater in this dissertation as well as the general field of demand response (DR). We use real time pricing (RTP) and variants of demand charge based rates to study dynamic pricing. 
Deterministic prices are those that don"t change over time. In demand response (DR), fixed price tariffs are quite common, where consumers are given a fixed schedule of price changes and can adjust accordingly. The average residential rate of electricity in the US is about $\$ 0.11$ per $\mathrm{kWh}$ according to the Electric Power Monthly report from the US Energy Information Administration (EIA). We use a standard flat rate of $\$ 0.1$ per $\mathrm{kWh}$ in the NTL model. Under the time-of-use (TOU) rate, time slots are designated as base, intermediate and peak operating hours with different rates as shown in Table 9. We assume a maximum regular price of $\$ 0.2$ per $\mathrm{kWh}$ and a minimum of $\$ 0.01$ per $\mathrm{kWh}$ in our simulation. Two variations of the TOU rates are considered for comparison: one has abrupt rate changes and the other has gradual rate changes between time slots. Figure 41 shows the abrupt and gradual TOU rates used in the model.

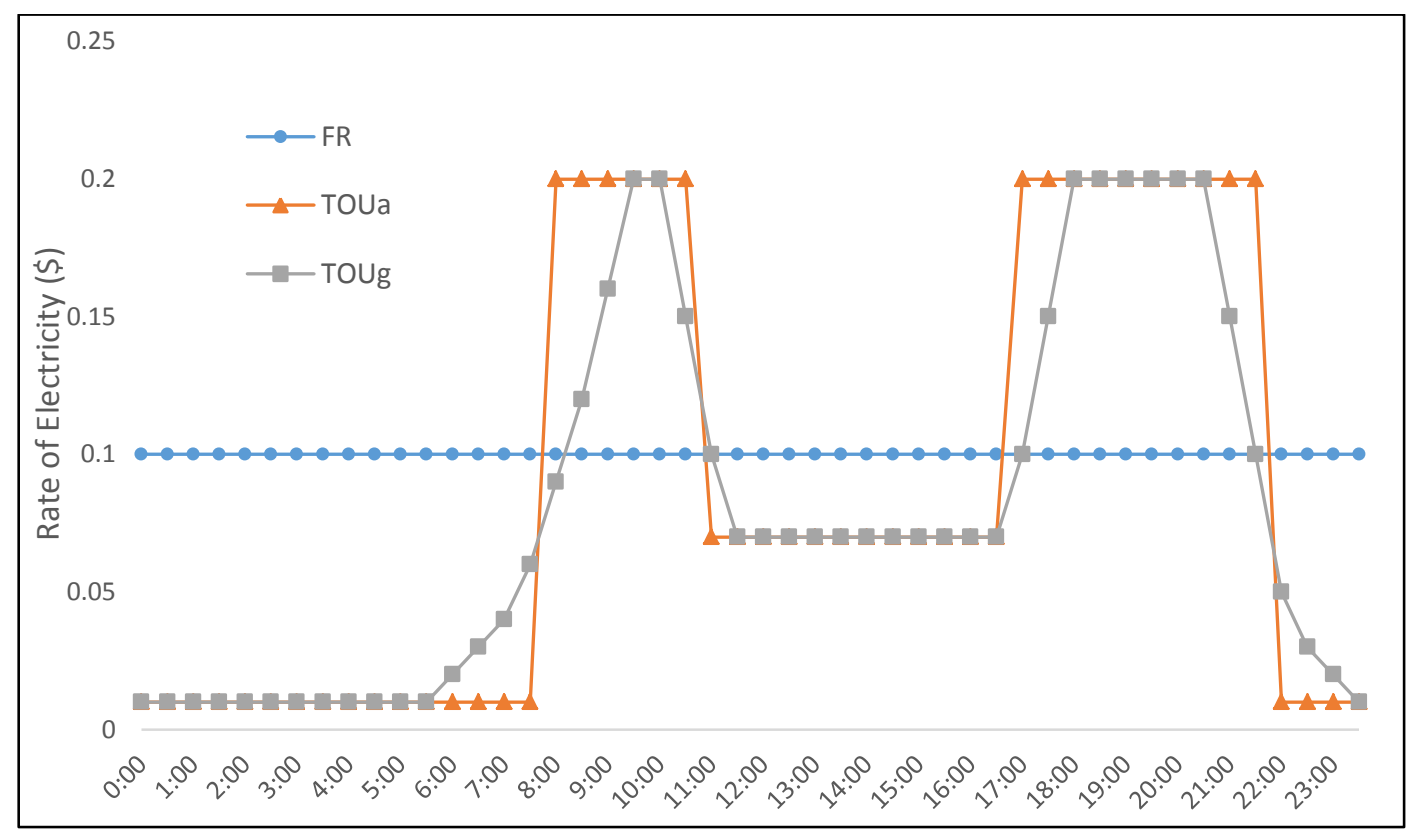

Figure 41: Static variable pricing structures: Flat rate, TOU abrupt, TOU gradual 
Table 9: TOU rates used in the NTL model

\begin{tabular}{|l|l|l|}
\hline Time Window & Load Type & Rate \\
\hline 0:00 $-8: 00$ & Base & $\$ 0.01$ \\
\hline 8:00 - 11:00 & Peak & $\$ 0.2$ \\
\hline 11:00-17:00 & Intermediate & $\$ 0.07$ \\
\hline 17:00-22:00 & Peak & $\$ 0.2$ \\
\hline 22:00-24:00 & Base & $\$ 0.01$ \\
\hline
\end{tabular}

In a dynamic variable pricing structure, the cost of electricity is stochastic in nature and changes based on various parameters of the system, for example load on the system, duopoly market, bidding process, etc. This constantly changing rate is communicated to the consumer via display systems or smart meters on a smart grid network. A simple method of dynamic real time pricing (RTP) is studied in order to compare consumers ${ }^{e e}$ responses to dynamic pricing. Since only HVAC loads and generic appliances are considered in the NTL/TCL models, we set the price of electricity as a function of energy usage as well as ambient temperature. Equation (57) is used to represent the RTP rate, where $p^{f}$ is the flat rate or base price and $p^{e}$ and $p^{t}$ are prices that are affected by energy usage and external temperature. $\Delta e$ and $\Delta t$ are the proportional differences in energy drawn and external temperature from some prespecified average values. We select $p^{f}=\$ 0.1, p^{e}=p^{t}=\$ 0.05$ and set the limits on the prices as $p_{\max }=\$ 0.25$ and $p_{\min }=\$ 0.01$. The possible rates are bound within these limits. When considering NTL, there is no effect of temperature on the load consumption, hence $p^{t}$ becomes zero and we set $p^{e}=\$ 0.1$. Also when considering TCL, we conduct a sensitivity analysis of including either the energy dependent part or the temperature dependent part by setting one of the coefficients to zero, or including both parts. 


$$
p=p^{f}+p^{e} \Delta e+p^{t} \Delta t
$$

Since the cost of electricity affects the energy usage pattern by consumers and that in turn affects the cost of electricity, it is uncertain what the rate might be in the next time interval. However, there exists a probability distribution of the electricity rates at each time interval on a stable system. Household owners will predict future rate changes based on experience. The proposed simulation model would accumulate a probability distribution table over time and use it to model the consumers learning ability. As discussed previously, in the event of uncertainty, we evaluate the total expected utility of the household given by the multi attribute utility function (MAUF).

In addition to the pricing models discussed above, we setup a separate experiment for the TCL model that includes flat rate, TOU and demand charge based rates. We have used the input variables specific to Glasgow, KY for the TCL model and hence design pricing mechanisms that correspond to the region. The standard flat rate for GEPB of $\$ 0.088 / \mathrm{kWh}$ is used as the base model. In order to make a valid comparison between different rate structures, we design the pricing models such that equivalent cost of electricity is comparable. We use a typical average summer load calculated from the consumption data of the 26 test houses in 2013 for June, July and August. In the design on the different rate structures, we ensure that the daily equivalent cost based on this typical summer load profile is comparable (Table 11).

The pricing structures used in the simulation experiment are given in Table 10. All the different rate structures considered here are based on the peak and off-peak periods as defined by Tennessee Valley Authority (TVA) for the Kentucky region. The 
On-Peak period is the six hour period from $1 \mathrm{PM}$ to $7 \mathrm{PM}$, while the rest of time is considered as Off-Peak. First of all, a static pricing using TOU is designed under this peak/off-peak designation as shown in the table. We also consider a standard flat rate with a time-of-use demand charge. This type of a rate structure is offered as an optional tariff by Louisville Gas and Electric (LG\&E) during summer period. In this pricing model a reduced standard rate is offered along with a demand charge assessed on the individual user peak for the month during each of the On-Peak and Off-Peak periods. Since the determination of this demand charge is dependent on the usage pattern and the peak load consumed by a user has considerable variance, we may consider this as a dynamic rate.

A novel method for residential electricity pricing is by using demand charge. We setup various pricing structures using demand charge in our experimental design. In addition to the standard rate that charges a consumer a fixed rate on the total $\mathrm{kWh}$ used during a billing cycle, a demand charge adds a high premium amount to the load consumed in one particular hour. This demand charge usually accounts for a significant portion of the bill, thus motivating people to reduce peak consumption. The demand charge can be applied to either the individual peak load consumed by the user or the coincident load consumed during system peak. The coincident load is the load consumed by a user during the hour in which the system load has the highest value for a billing cycle. As shown in Table 10, demand charge on coincident peak and demand charge on user peak are designed with a high demand charge $(\$ 12 / \mathrm{kW})$ and a low demand charge $(\$ 5 / \mathrm{kW})$ for each. The standard rates are adjusted accordingly to maintain a comparable equivalent daily cost. A special rate structure that uses TOU rates with demand charge is 
also considered, called the Infotricity rate. Currently, the Glasgow Electric Plant Board (GEPB) is implementing a more complicated form of the Infotricity rate to achieve system level peak reduction. GEPB is using a host of direct load control mechanisms on a limited number of participating houses to study the preliminary effects of this new pricing scheme. However, the remaining homes that are not directly controlled by the utility are also affected by the new rates. We include this novel pricing model in our study to verify the effects on system load due to this decentralized behavior.

Table 10: Differential pricing models and rate designs

\begin{tabular}{|c|c|c|c|c|c|c|}
\hline Rate Type & $\begin{array}{c}\text { Standard } \\
\text { Rate } \\
(\$ / \mathbf{k W h})\end{array}$ & $\begin{array}{c}\text { On Peak } \\
\text { Rate } \\
(\$ / \mathbf{k W h})\end{array}$ & $\begin{array}{c}\text { Off Peak } \\
\text { Rate } \\
(\$ / \mathbf{k W h})\end{array}$ & $\begin{array}{c}\text { Demand } \\
\text { Charge } \\
(\$ / k W)\end{array}$ & $\begin{array}{c}\text { On } \\
\text { Peak } \\
\text { DC } \\
(\$ / k W)\end{array}$ & $\begin{array}{c}\text { Off } \\
\text { Peak } \\
\text { DC } \\
(\$ / k W)\end{array}$ \\
\hline Flat Rate & 0.08800 & & & & & \\
\hline DC on Coincident Load 1 & 0.07638 & & & 5 & & \\
\hline DC on Coincident Load 2 & 0.06200 & & & 12 & & \\
\hline DC on User Peak 1 & 0.07638 & & & 5 & & \\
\hline DC on User Peak 2 & 0.06200 & & & 12 & & \\
\hline TOU & & 0.16 & 0.052 & & & \\
\hline Standard with TOU DC & 0.056 & & & & 12.68 & 3.25 \\
\hline Infotricity & & 0.12 & 0.04 & 10.67 & & \\
\hline
\end{tabular}

Table 11: Daily equivalent costs for different pricing models using typical summer load

\begin{tabular}{|l|c|}
\hline Rate Type & $\begin{array}{c}\text { Daily Equivalent Cost } \\
\text { (with Typical Summer Load) }\end{array}$ \\
\hline Flat Rate & 5.541058117 \\
Demand Charge on Coin Load 1 & 5.45214937 \\
Demand Charge on Coin Load 2 & 5.438998856 \\
Demand Charge on User Peak 1 & 5.45214937 \\
Demand Charge on User Peak 2 & 5.438998856 \\
TOU & 5.520060566 \\
Standard with TOU DC & 5.530379398 \\
Infotricity & 5.540005248 \\
\hline
\end{tabular}




\subsection{NTL UNDER STATIC PRICING}

The simulation analysis is divided into four sections dealing with the NTL and TCL models under static and dynamic pricing as we have established earlier. Here we look into the effect of static variable pricing on the usage behavior of NTL appliances. As described previously, the load factor (LF) and load variation (VAR) are the considered the key parameters in our solution analysis. Although the NTL model is defined for just a 24 hour period, the randomness of various parameters in the model accounts for these output parameters to fluctuate slightly over each day. The probability distribution of the electricity rates also becomes more accurate with the inclusion of more data points as the days progress. We will use the cumulative moving average of these values at the end of the simulation run for our analysis.

Recall that we include three appliances in our NTL model: washer, dryer and dishwasher. The load profiles from the simulation experiments pertaining to these loads are studied in two forms. The first one is a load profile attributed to just the three appliances being studied. This profile helps us to observe how the actual load is shifted to different times as a result of the TOU rates. However, by just looking at these three appliances ${ }^{\mathrm{ee}}$ loads does not provide an accurate data for the effect of demand response on the total residential load. Therefore, the second load profile we study includes loads from not only these three appliances but remaining typical residential loads. The latter is estimated by studying a typical residential load profile (NAHB Research Center, Ltd., 2001). Although not exact, this estimation is important to our analysis on the effect of the appliances load shift in the context of total household load. 
We study the effect of TOU rates against a standard flat rate model. Along with the abrupt and gradual TOU rates model, we also run a cost-minimizing model in which all the households give zero weight to the utility of convenience. In other words studying the cost-minimizing model helps us to better understand the effect of including the convenience in the multi-attribute utility function. Because vast majority of the literature uses cost minimization only, we expect to see different effect of demand response or TOU after including the inconvenience in the utility function.

Figure 42 shows the different load profiles for the three appliances in response to various cost models. The flat rate model is one where the cost of electricity is maintained a uniform rate of $\$ 0.07$, and the two TOU models are implemented with the rate structures discussed above in Section 5.2. The cost-minimizing model implements the gradual TOU rate but the utility functions for the households only have the cost component while zero weightage is given to convenience. First, we observe a distinctive difference between the TOU rates and the cost-minimizing-only model. Based on the rate structure, all the households changed their usage to the off-peak time in the costminimizing-only model since no consideration was given to convenience. On the other hand, in the TOU models, we see that some portion of the households shifted their load from the evening period to morning period. This is what we would expect to see since the rates are cheaper in the intermediate load period but the convenience is still quite high. Second, the fact that not all households changed their usage to the morning period indicates the varying behavior of the population based on their utility functions. Finally, between the two variations of TOU rates, the gradual rate structure seems to be better since it has a lower peak than that of the abrupt rate structure. 


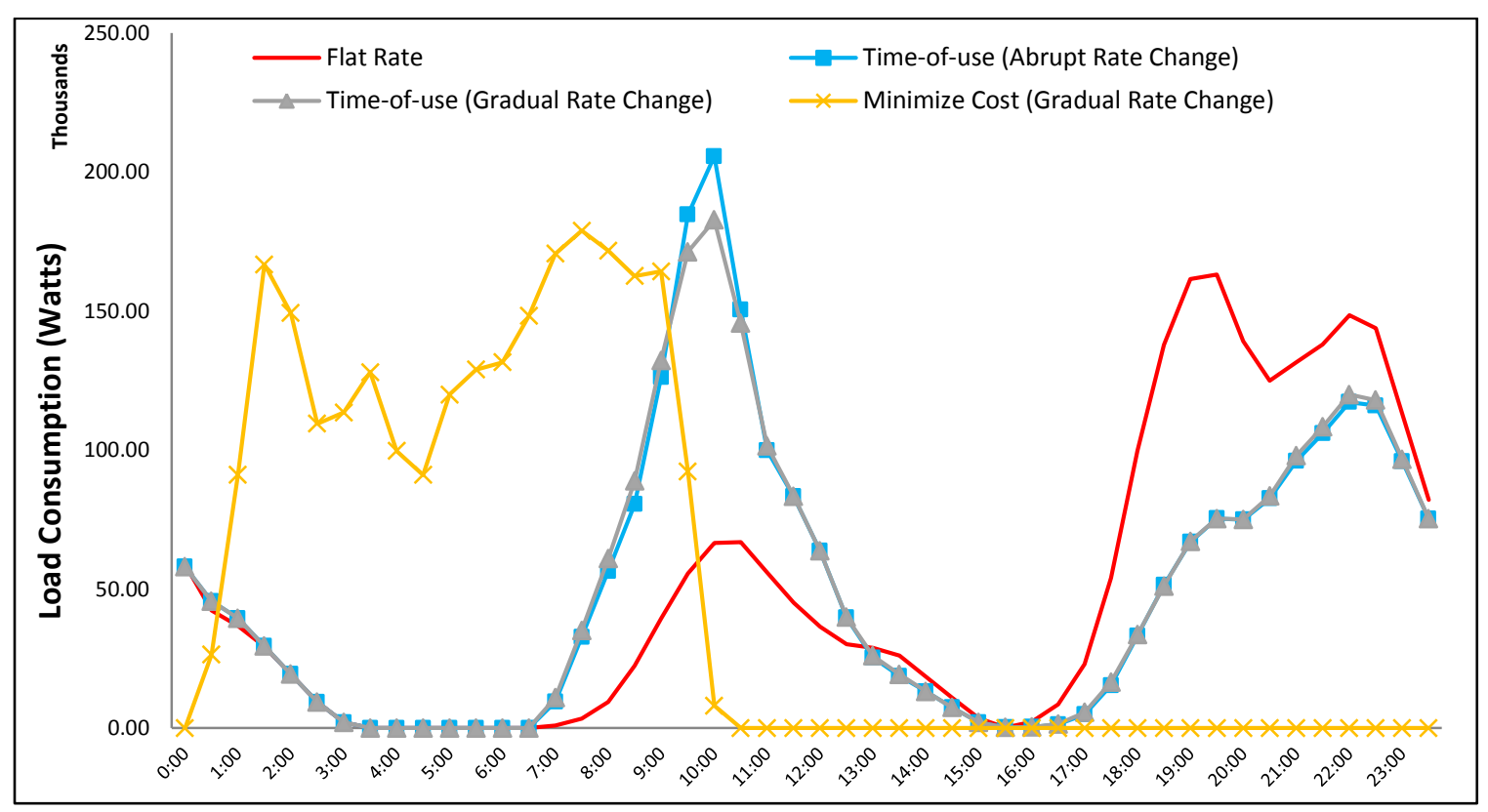

Figure 42: Hourly load profiles for the three appliances

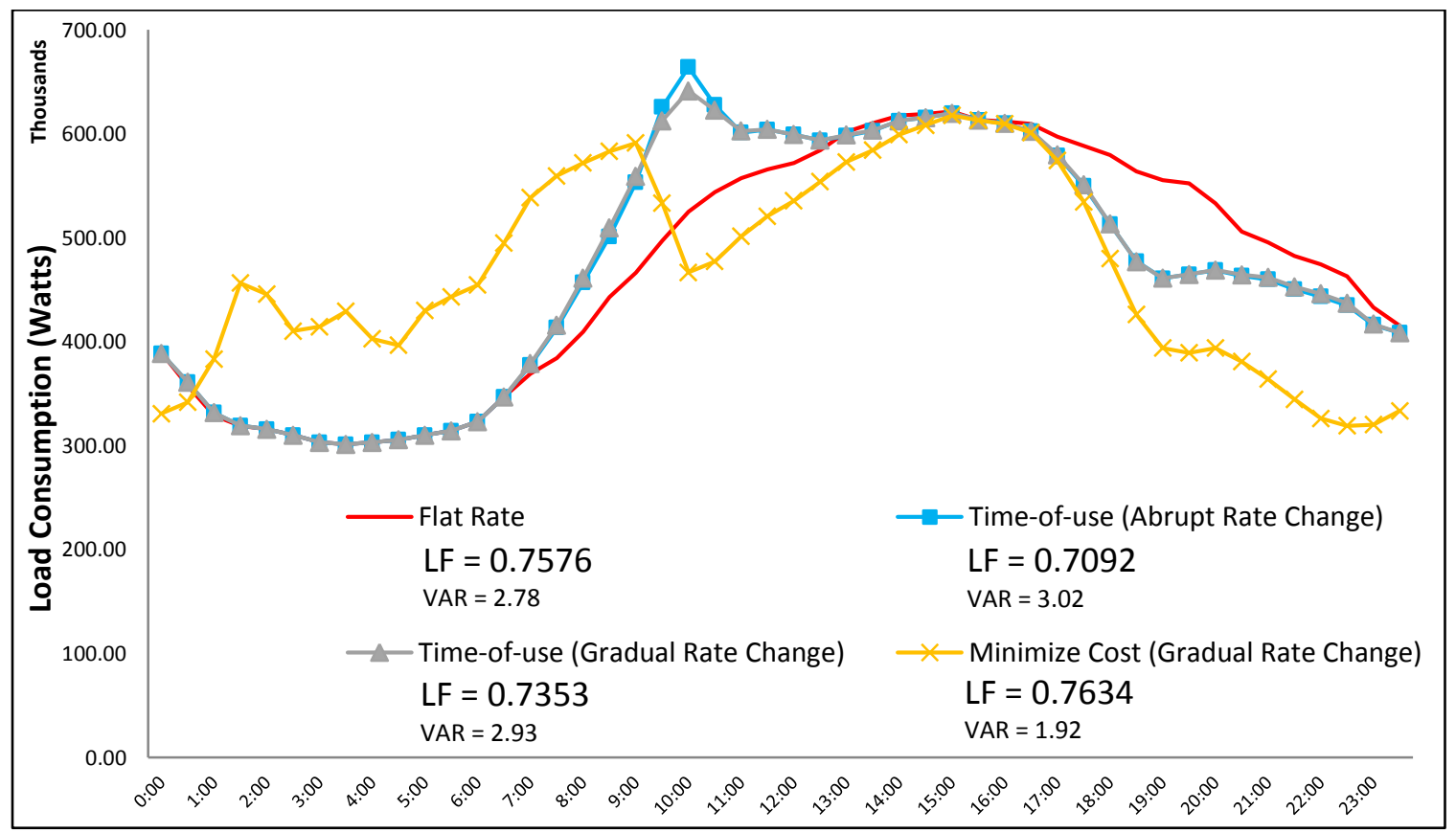

Figure 43: Hourly total load profiles for NTL model 
Figure 43 displays the load shifts in the context of the total load. The cost minimizing only model seems to have the highest LF (0.7634) and lowest load variation (1.92) when compared to the other models. Again, this is because the cost-minimizing model does not consider convenience to the consumer. As a matter of fact, as soon as we introduce the utility of convenience with the TOU models, we see a decrease in LF and increase in load variation. This is an indication that cost minimizing functions that do not consider convenience factors overestimate the benefits of the DSM. The inclusion of convenience in modeling such consumer behavior can provide closer to realistic, even though not necessarily better, analysis of the system.

Recall that there are three types of household in our simulation model: low-, medium- and high-income households. We study how they respond differently to the demand response. Table 12 shows the average cost in dollar amount per household for using the three appliances for a day as well as the average inconvenience experienced during that period. From Table 12, the average inconvenience and cost for the three household categories, when compared between the flat rate and time-of-use rate models, clearly verifies the varying characteristics of consumers as defined by their utility functions. First, the average cost and inconvenience is almost same for all the groups in the flat rate model, since there is no behavioral change when the rate of electricity is constant throughout the day. Second, under the two time-of-use rates, we observe a decrease in cost for group A (cost-favoring) and an increase in cost for group C (comfort-favoring). This is as expected because the comfort-favoring group (high income households) will prefer to pay more for higher convenience and the cost-favoring group (low income households) will prefer cost savings in exchange for less convenience. The 
average inconvenience values also support this argument. Third, we see higher inconvenience value for group A while group $\mathrm{C}$ sustains a relatively low inconvenience. Finally, the cost minimizing model, however, results in the lowest average cost for all the groups, but the average inconvenience is increased up to 4.6. Since there is no inclusion of convenience in the cost minimizing model, the households prefer to reduce their total cost without any regard for the ,convenience ${ }^{e e}$ of use.

Table 12: Average cost and inconvenience comparison between flat rate and TOU rates

\begin{tabular}{|l|l|l|l|l|l|}
\hline \multicolumn{2}{|c|}{} & $\begin{array}{l}\text { Flat } \\
\text { Rate } \\
\text { Model }\end{array}$ & $\begin{array}{l}\text { Time-of-use } \\
\text { (Abrupt } \\
\text { Change) }\end{array}$ & $\begin{array}{l}\text { Time-of-use } \\
\text { (Gradual } \\
\text { Change) }\end{array}$ & $\begin{array}{l}\text { Cost } \\
\text { Minimize }\end{array}$ \\
\hline \multirow{2}{*}{$\begin{array}{l}\text { Average Cost } \\
\text { (\$/day/household) }\end{array}$} & Group $\boldsymbol{A}$ & 0.8665 & 0.7490 & 0.7486 & 0.6465 \\
\cline { 2 - 6 } & Group $\boldsymbol{B}$ & 0.8811 & 0.8762 & 0.8706 & 0.6528 \\
\hline \multirow{2}{*}{$\begin{array}{l}\text { Average } \\
\text { Inconvenience } \boldsymbol{C}\end{array}$} & 0.8608 & 0.9215 & 0.9168 & 0.6402 \\
\cline { 2 - 6 } & Group $\boldsymbol{A}$ & 0 & 1.13 & 1.159 & 3.93 \\
\cline { 2 - 6 } & Group B & 0 & 0.678 & 0.676 & 4.611 \\
\hline
\end{tabular}

\subsection{NTL UNDER DYNAMIC PRICING}

We consider a load based real-time model for the dynamic variable pricing. Under the dynamic load based rate structure, the simulation model has to record past data regarding the rates in order for the household to predict the rates at different time windows. We use only ten days of past data, successively averaged, in order to get a good probability estimate. By taking the successive average, we are giving high importance to recent data and low importance to older data. Let us consider a particular time interval 
$t$ on day $d+1$, for which we want to know the probability distribution of electricity prices. Also for $i=\{1,2,3 \ldots 25\}$ and $j=\{\mathrm{d}, \mathrm{d}-1, \mathrm{~d}-2 \ldots \mathrm{d}-9\}$ let,

$$
c_{j}^{i}=\left\{\begin{array}{rr}
1 & \text { if rate } i \text { had occured on day } j \\
0 & \text { otherwise }
\end{array}\right.
$$

The successive average of the occurrence (or frequency) of rate $i$ can be expressed as,

$$
S C^{i}=\frac{\left(10 c_{d}^{i}+9 c_{d-1}^{i}+8 c_{d-2}^{i}+\cdots+c_{d-9}^{i}\right)}{(10+9+8+\cdots+1)}=\frac{C^{i}}{55}
$$

where, $C^{i}=\left(10 c_{d}^{i}+9 c_{d-1}^{i}+8 c_{d-2}^{i}+\cdots+c_{d-9}^{i}\right)$, is the successively weighted sum of the binary counts $c_{j}^{i}$.

When implementing this successive average in Equation (58), instead of using the binary variable to count, we set the initial count at day $d$ equal to 10 and reducing this value by 1 for every day that has passed until it is exhausted to 0 . The probability of occurrence of rate $i$ during the current time period $t$ of day $d+1$ is thus calculated as,

$$
p_{d+1}^{i}=\frac{S C^{i}}{\sum_{i=1}^{25} S C^{i}}=\frac{C^{i}}{\sum_{i=1}^{25} C^{i}}
$$

Two matrix arrays (see Figure 44) are defined with identical rows and columns, where each of the 48 rows corresponds to a half-hour time window and 25 columns record possible rates from $\$ 0.01$ to $\$ 0.25$ during the specific time window. One of the tables (CTable) is used to count the occurrence $c_{j}^{i}$, and the other table (PTable) is used to establish a probability distribution based on the first. For instance, if the rate of electricity is $\$ 0.07$ at $8: 00$, the first table will increment the corresponding cell of the table by 10 , each day reducing by one until it becomes zero. This way we can keep track of the rates 
at different time intervals and thus establish a probability distribution for these rates using the successive average method.

An example of the CTable and PTable can be seen in Figure 44, where the CTable consists of an arbitrary count and the PTable is calculated accordingly. Since the probability of the rates is updated periodically and all the data of the rates is stored in CTable throughout the simulation run, we are able to model the probabilistic forecasting behavior of the consumers when evaluating their utility functions. We assume that in a stable system, the probability distribution of rates is exact and is provided by the energy provider via smart meters.

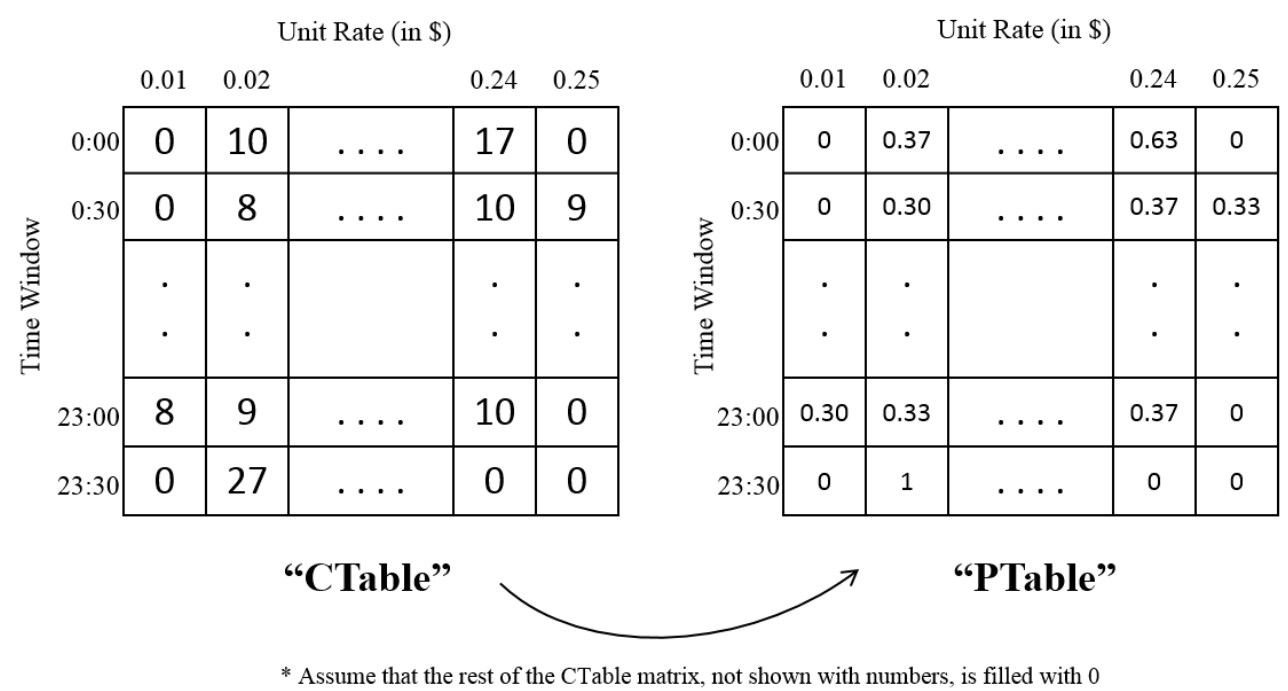

Figure 44: Example calculation of probabilities in PTable from CTable

Since the time window for utility evaluation is 30 minutes, we must evaluate the rate of electricity based on system load every 30 minutes. This will simulate a dynamic rate structure that fluctuates every half hour and it is assumed that the consumer is notified of this real time rate through smart meters. A dummy entity is created every 30 minutes and is used to update the CTable accordingly. The purpose of this default entity 
is to update the values of the rate $X$ and CTable. When the dummy entity exits the source, a state assignment is made. The entity updates the state variable $X$ according to the cost function. Just before it is destroyed, the entity updates the variable CTable as follows.

CTable $[(2 *$ TimeOfDay $)+1,100 X]=$ CTable $[(2 *$ TimeOfDay $)+1,100 X]+10$ Another dummy entity is created every day in order to update the PTable. In another source object called "SetPTable", by offsetting the initial creation time by 24 hours and having an inter-arrival time of 24 hours, we can model the PTable to be updated at the end of each day. As soon as the entity exits the source, a process is triggered which essentially updates the probability distribution of rates by running Algorithm 3 .

Algorithm 3. Updating PTable in Simio

Step 1: Initialize $i=1$ and $j=1$

Step 2: PTable $[\mathrm{i}, \mathrm{j}]=$ CTable $[\mathrm{i}, \mathrm{j}]$ / (CTable[i, 1]+CTable[i, 2]+....+CTable[i, 25])

Step 3: If $\mathbf{j}<25$, then $j=\mathbf{j}+1$ and goto Step 2, Else goto Step 4

Step 4: If $\mathrm{i}<48$, then $i=\mathrm{i}+1, j=1$ and goto Step 2, Else goto Step 5

Step 5: Stop

Since the NTL appliances are not affected by temperature, we exclude the temperature coefficient from the real-time price in Equation (57), and thus reduce it to Equation (60). This new cost equation contains only the energy dependent coefficient $p^{e}$ and the fixed base rate $p^{f}$.

$$
p=p^{f}+p^{e} \Delta e
$$

The total run time for the simulation model is set to be 10 days allowing for 5 days of warm-up period to initialize the probability of the rates (PTable). Figure 45 illustrates how the price is set over several days. In order to estimate the number of replications 
required for a sensitivity analysis, we ran the model with an initial number of 25 replications. A 95\% confidence interval on the average energy level at each time interval was studied and the half width $h$ and standard deviation $s$ at each interval were recorded. The correct number of replications required was then estimated using Equation (61). For any of the time intervals, the number of replications required did not exceed the initial value of 25 .

$$
h=t_{n-1} \frac{s}{\sqrt{n}}
$$

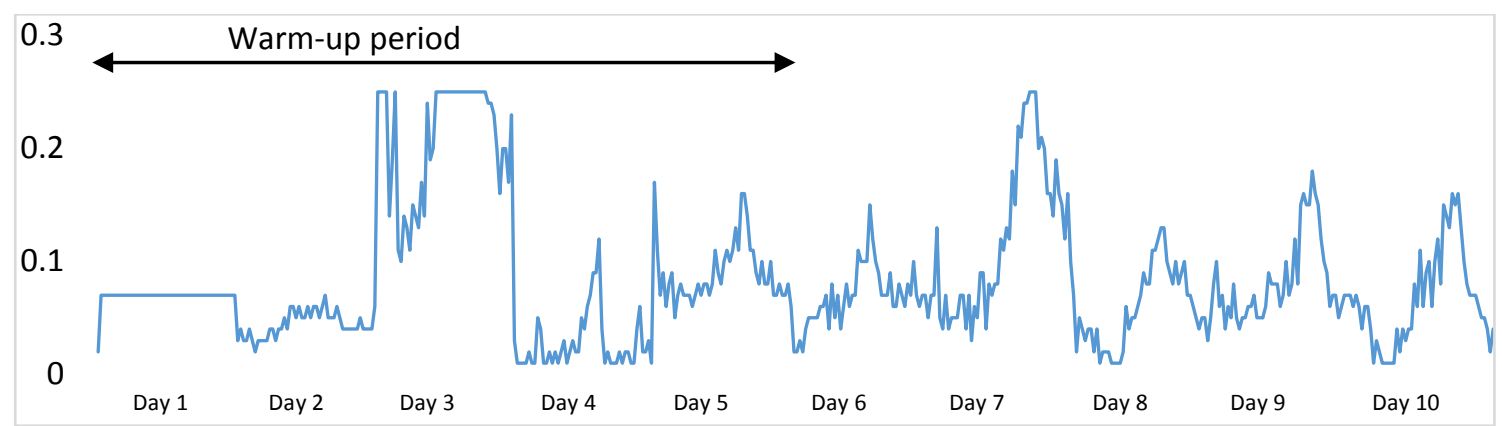

Figure 45: Simulation of electricity rates over time based on RTP

In our experiments on NTL under real time pricing, we first set $p^{f}=0.1$ and $p^{e}=0.1$, and studied the response of the individual groups by comparing flat rate and TOU. As shown in Table 13, we observe that both LF and VAR are slightly improved by applying the real time price. The increase of LF is about $0.05 \%, 1.48 \%$ and $0.42 \%$ for Group A, B and C, respectively. We then conducted a sensitivity analysis by changing the values of $p^{f}$ and $p^{e}$, the results of which are shown in Table 14. We observe that, for a given value of $p^{f}, \mathrm{LF}$ and VAR are improving as $p^{e}$ increases. Similarly, for a given value of $p^{e}$, LF is also improving as $p^{f}$ increases, but the same does not hold for VAR. The best value of LF (0.6336) and VAR (3.59) in this analysis is obtained at higher 
coefficients of $p^{f}(0.12)$ and $p^{e}(0.14)$. The load usage behavior of NTL appliances seem to be highly affected by cost from this analysis. As the rate structure is given higher coefficients, the consumers tend to reduce their impact on the system load, at least from the view point of flexible NTL appliances. Further experimental design can be done on improved real-time structures to get a better understanding, however we prioritize TCL loads as having higher impact based on dynamic rates and focus on those in detail.

Table 13: Group-wise response comparison between flat rate and RTP

\begin{tabular}{|l|c|c|c|c|}
\hline & \multicolumn{2}{|c|}{ LF } & \multicolumn{2}{c|}{ VAR } \\
\cline { 2 - 5 } & Flat Rate & RTP & Flat Rate & RTP \\
\hline Group A & 0.5942 & 0.5949 & 3.9868 & 3.6564 \\
Group B & 0.5937 & 0.6085 & 3.9877 & 3.8572 \\
Group C & 0.5942 & 0.5984 & 3.9868 & 3.9739 \\
\hline
\end{tabular}

Table 14: Sensitivity analysis of RTP for NTL

\begin{tabular}{|c|c|c|c|}
\hline $\mathbf{p}^{\mathbf{f}}$ & $\mathbf{p}^{\mathbf{e}}$ & $\mathbf{L F}$ & $\mathbf{V A R}$ \\
\hline 0.1 & 0.06 & 0.6157 & 3.85618 \\
0.1 & 0.1 & 0.6215 & 3.73087 \\
0.1 & 0.14 & 0.6328 & 3.60504 \\
0.08 & 0.06 & 0.6171 & 3.83114 \\
0.08 & 0.1 & 0.6215 & 3.73508 \\
0.08 & 0.14 & 0.6311 & 3.61489 \\
0.12 & 0.06 & 0.6176 & 3.83609 \\
0.12 & 0.1 & 0.6222 & 3.72435 \\
0.12 & 0.14 & 0.6336 & 3.5897 \\
\hline
\end{tabular}

\subsubsection{Experimental Design (Latin Hypercube Method)}

The scope for experimental design and analysis for selecting appropriate price functions in order to obtain a desirable improvement in LF is very good. Cost functions can de designed by studying regression models based on statistical analyses, using a large 
sample of data points generated from our simulation model. An immediate work in this proposed research is to conduct an experimental design in order to facilitate a pricing selection, both under deterministic and real time settings. In this section, we discuss a small example where we used the Latin Hypercube method to do a factorial analysis on the TOU rates.

Using the NTL simulation model, we conducted a factorial analysis of the model in order to optimize the rate structure to obtain minimum load variation and maximum LF. In order to maintain consistency among both output variables, we use the inverse of LF (also known as Peak-to-Average Ratio or PAR), so that we can minimize both these values. We designed experiments with five and ten factors respectively. In the five factor DOE, we consider the rates during the base, intermediate and peak hours to be a factor and the coefficient of cost $k_{1}$ in the utility function for the high and low income households to be factors. The coefficient for convenience $k_{2}$ is automatically equal to $\left(1-k_{1}\right)$. We do not consider $k_{1}$ for medium income households be a factor because it is assumed to be 0.5 . In the ten factor DOE, we divide the total time of the day into eight equal parts of three hour intervals and consider the rates during each of these time periods to be a factor. The coefficient of cost $k_{1}$ for group A and group $\mathrm{C}$ are the two remaining factors.

For simplicity of the initial experiment, the levels of the factors are set to be 11. In this example, we limit the values of the rates to be between $\$ 0.03$ and $\$ 0.13$, as per the standard residential service provided by Louisville Gas and Electric Company (LG\&E). This range can be changed as desired for other studies based on residential rates for other regions. The values of $k_{1}$ for group A will always be more than or equal to 0.5 , and for 
group $\mathrm{C}$ will always be less than or equal to 0.5 . As indicated earlier, the output responses in both five and ten factor DOE are PAR (inverse of LF) and VAR. We set up the experiment by using a Latin Hypercube Design with 11 design points. The experiment results are analyzed using the response surface method in MiniTab.

The estimated regression coefficients for PAR and VAR obtained from the five factor DOE is shown in Table 15 below, where $R_{1}, R_{2}$ and $R_{3}$ correspond to the rates during base, intermediate and peak hours. We then used the response optimizer to minimize the two responses PAR and VAR, and obtained the optimal values for the three rates, reported in the "Optimal Values" Column in Table 15. In summary, the optimal rates for base, intermediate and peak periods are found to be $\$ 0.03, \$ 0.03$ and $\$ 0.13$, respectively. This suggests that the distinction between the base and intermediate period have negligible effect on consumer's behavior but the peak period has a high effect. This is consistent with the peak/off-peak period distinction adopted by many utility where a separate intermediate period is often not considered. However, this result is based on flexible NTL appliances and its effect on the TCL model may be different.

Similar regression and optimization analysis was also done for the ten factor DOE. The estimated regression coefficients and optimal rates are shown in Table 16. Particularly, $T_{1}$ to $T_{8}$ are the rates for the three hour time intervals, and their optimal values are found to be $\$ 0.0782, \$ 0.03, \$ 0.03, \$ 0.13, \$ 0.13, \$ 0.03, \$ 0.03$ and $\$ 0.03$, respectively. Finally, we note that the scope for the experimental design is much greater than discussed here and we will leave this section for further research. The use of factorial analysis and optimization via simulation can potentially provide robust rate structures in helping in system-wide load leveling. 
Table 15: Estimated regression coefficients for PAR and VAR for five factor DOE

\begin{tabular}{|l|l|l|l|}
\hline Terms & PAR & VAR & Optimal values \\
\hline Constant & -5.7766 & -0.7031 & \\
$\mathrm{R}_{1}$ & 28.0461 & 2.8631 & $\$ 0.03$ \\
$\mathrm{R}_{2}$ & 25.3423 & 2.4308 & $\$ 0.03$ \\
$\mathrm{R}_{3}$ & 30.2601 & 3.3078 & $\$ 0.13$ \\
\hline
\end{tabular}

Table 16: Estimated regression coefficients for PAR and VAR for ten-factor DOE

\begin{tabular}{|l|l|l|l|}
\hline Terms & PAR & $\begin{array}{l}\text { Load } \\
\text { Variation }\end{array}$ & \\
\hline Constant & 2.1381 & 0.1465 & \\
$\mathrm{~T}_{1}$ & -27.6781 & -1.9669 & $\$ 0.0782$ \\
$\mathrm{~T}_{2}$ & -1.1671 & -1.4468 & $\$ 0.03$ \\
$\mathrm{~T}_{3}$ & 1.1435 & 0.0725 & $\$ 0.03$ \\
$\mathrm{~T}_{4}$ & -2.2538 & -0.345 & $\$ 0.13$ \\
$\mathrm{~T}_{5}$ & -4.5566 & -0.5926 & $\$ 0.13$ \\
$\mathrm{~T}_{6}$ & 1.5383 & 0.2476 & $\$ 0.03$ \\
$\mathrm{~T}_{7}$ & 3.1187 & 0.3906 & $\$ 0.03$ \\
$\mathrm{~T}_{8}$ & 3.9723 & 0.6938 & $\$ 0.03$ \\
\hline
\end{tabular}

\subsection{TCL UNDER STATIC PRICING}

The effect of thermostatic load is more dynamic in nature as the amount of load consumed is affected by the weather conditions. The impact of demand response is not as apparent as with the NTL since monetary incentive alone is not enough to promote load reduction. The thermal discomfort experienced by the household as a result of load shift and ambient weather is considered as the trade-off factor against the actual cost of electricity usage. In contrast to the flexible NTL appliances considered in the previous sections, the TCL model represents a more dynamic system. First of all we study the effects of static variable pricing.

Based on the characteristics of different households, as defined by their utility functions, the simulation results suggest varying responses in terms of HVAC usage and 
the resulting average room temperature. Figure 46 shows the temperature response of a risk-neutral low-income household with a maximum allowable discomfort tolerance of $5^{\circ} \mathrm{F}$. First, we compare the household"s response to three flat rates of $\$ 0.1$ and $\$ 0.1 \pm 0.02$. Since the household has a high utility for cost, the higher the flat rate the higher the average temperature thus the higher degree of discomfort consumer would experience in order to save cost. For example, when the flat rate price is reduced to $\$ 0.08$, we can see a reduction in average room temperature. The discomfort is reduced to an average of $4.09^{\circ} \mathrm{F}$ as compared to $4.87^{\circ} \mathrm{F}$ obtained at a rate of $\$ 0.1$. Second, when the TOU rates are applied, this household takes advantage of the low cost by using most of the AC load during the low cost periods. This is evidenced by a valley of room temperature between $t=0.5$ and $t=6$.

Similarly, Figure 47 shows that the AC command signals drop off during the peak periods, which is expected from cost-favoring consumers. Additional results from the simulation reveal that in comparison to the flat rate, the abrupt TOU rates resulted in a cost reduction from $\$ 0.497$ to $\$ 0.491$ per day, as well as discomfort reduction from $4.87^{\circ} \mathrm{F}$ to $3.92^{\circ} \mathrm{F}$. This is because TOU rates allow this household to have lower room temperatures during off-peak periods while keeping a higher temperature (maximum of $5^{\circ} \mathrm{F}$ higher than the preferred temperature) during all other periods. The gradual TOU only produced a discomfort reduction from $4.87^{\circ} \mathrm{F}$ to $4.03^{\circ} \mathrm{F}$, while keeping the cost same as that with flat rate. When compared with the flat rate, abrupt TOU produces better cost savings and discomfort reduction than gradual TOU. The thermal dynamics of the house causes slow temperature changes due to wall insulation, thermal resistance, thermal mass property and overall gradual heat exchange with ambient environment. Intuitively, abrupt 
TOU allows the household to run the $\mathrm{AC}$ up to the last time interval before the rate increases, and thus causes the room to be cooler for a longer period without using energy during peak period. In the gradual TOU rates, the household begins to gradually reduce AC usage, as seen in Figure 47 at around $6^{\text {th }}-7^{\text {th }} \mathrm{hr}$, corresponding to gradual increase in price. This causes the room to heat up earlier than the abrupt TOU case.

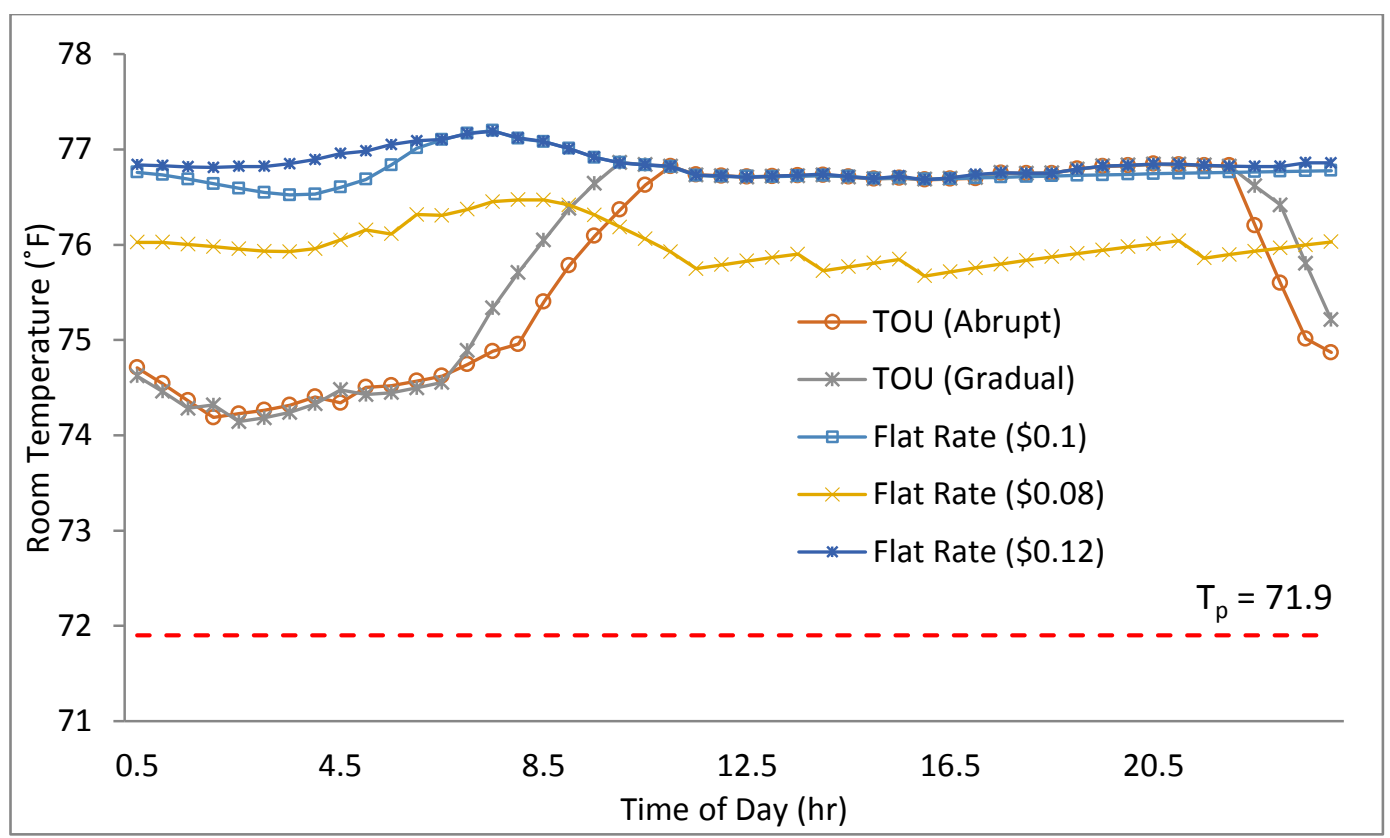

Figure 46: Temperature response for a low income (cost-favoring) household

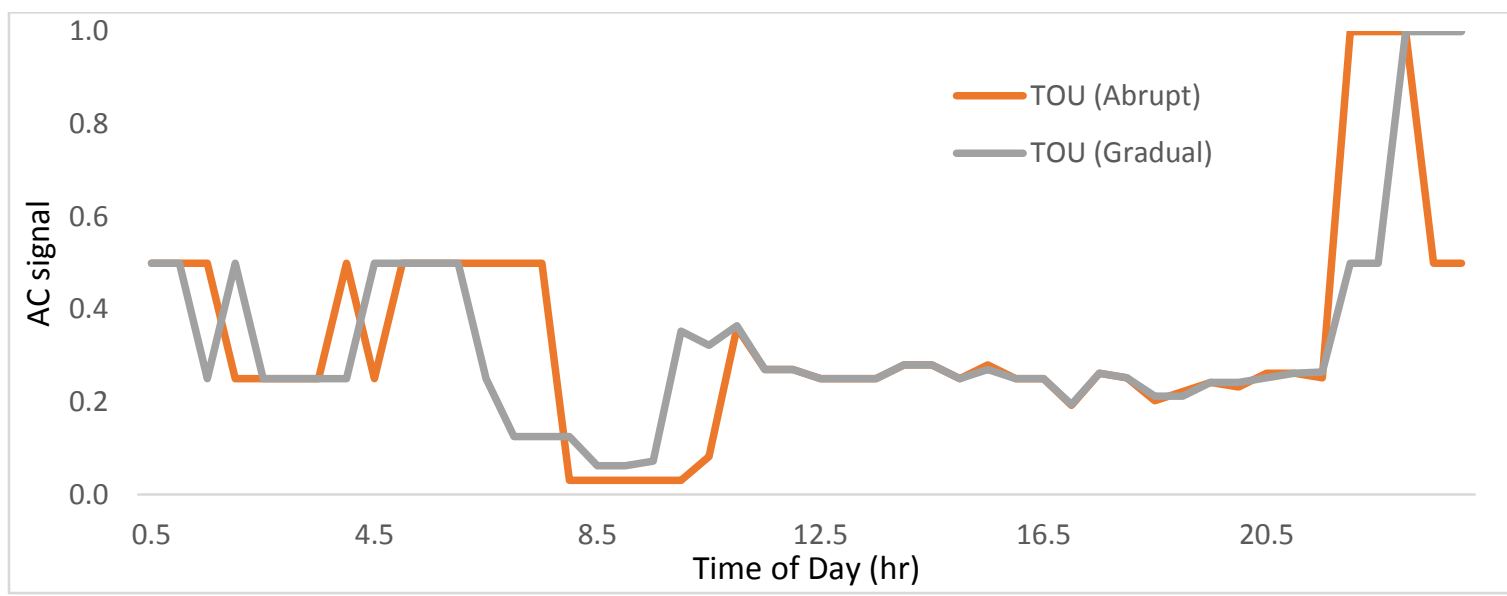

Figure 47: AC command signals for a low income (cost-favoring) household 
On the other hand, Figure 48 and Figure 49 show how medium and high income households respond differently to the TOU rates. The flat rate of $\$ 0.1$ yields an average discomfort of $2.84^{\circ} \mathrm{F}$ and $0.24^{\circ} \mathrm{F}$ for the medium (Group B) and high income (Group C) households, respectively (vs. $4.87^{\circ} \mathrm{F}$ for low income household). At a reduced flat rate of $\$ 0.08$, Group B household further reduced its discomfort to $1.83^{\circ} \mathrm{F}$, while that of the Group C household stayed at $0.24^{\circ} \mathrm{F}$. The comfort-favoring customer displayed little response and maintained a minimum deviation from preferred temperature. When applying the TOU rates, Group B observed a cost reduction from $\$ 0.84$ (with flat rate) to $\$ 0.66$ and a lower discomfort of $2.4^{\circ} \mathrm{F}$. Group $\mathrm{C}$ was again least affected by the TOU rates as shown by the temperature profile in Figure 49. The cost remained at a high value of $\$ 1.02$ and the discomfort remained very low around $0.25^{\circ} \mathrm{F}$.

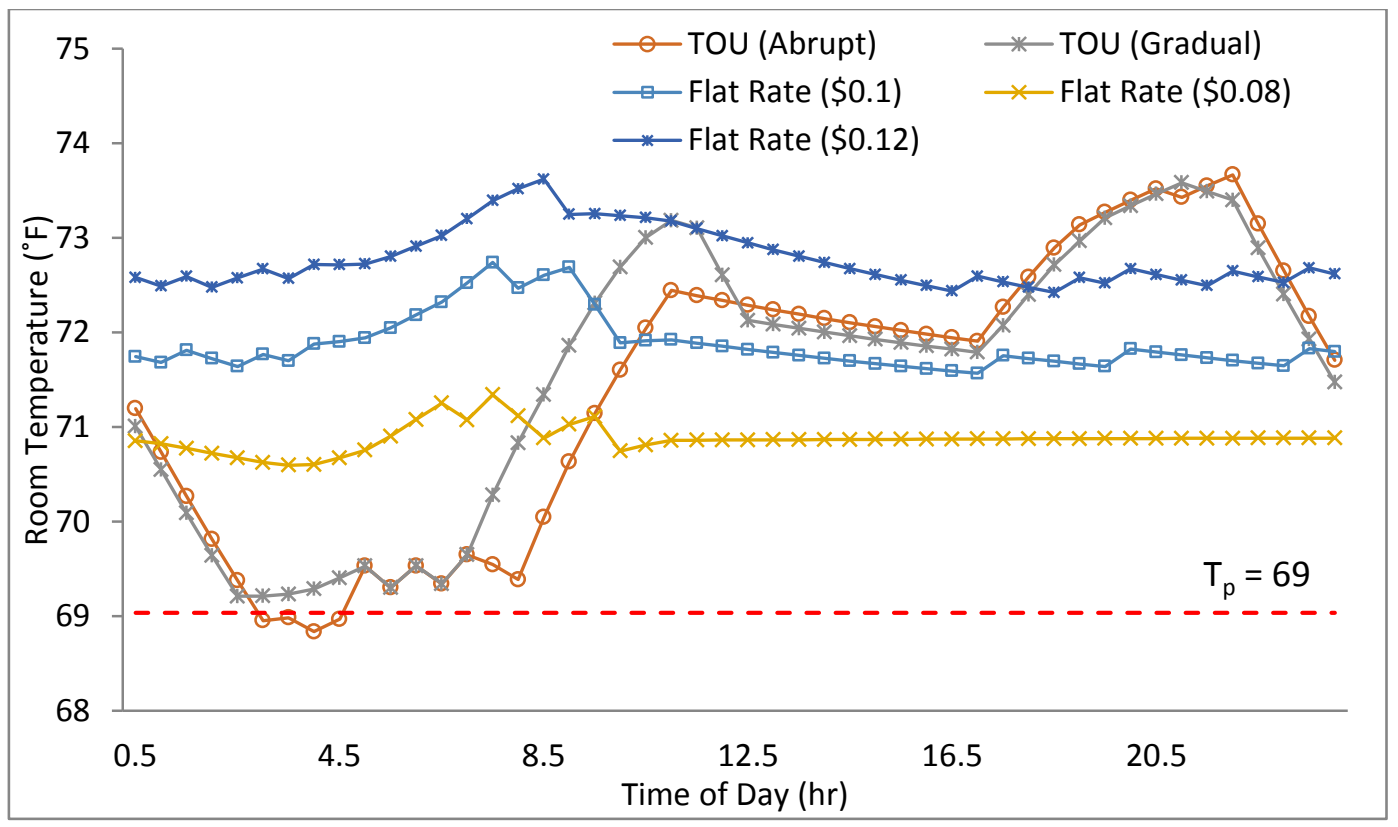

Figure 48: Temperature response for a medium income household 


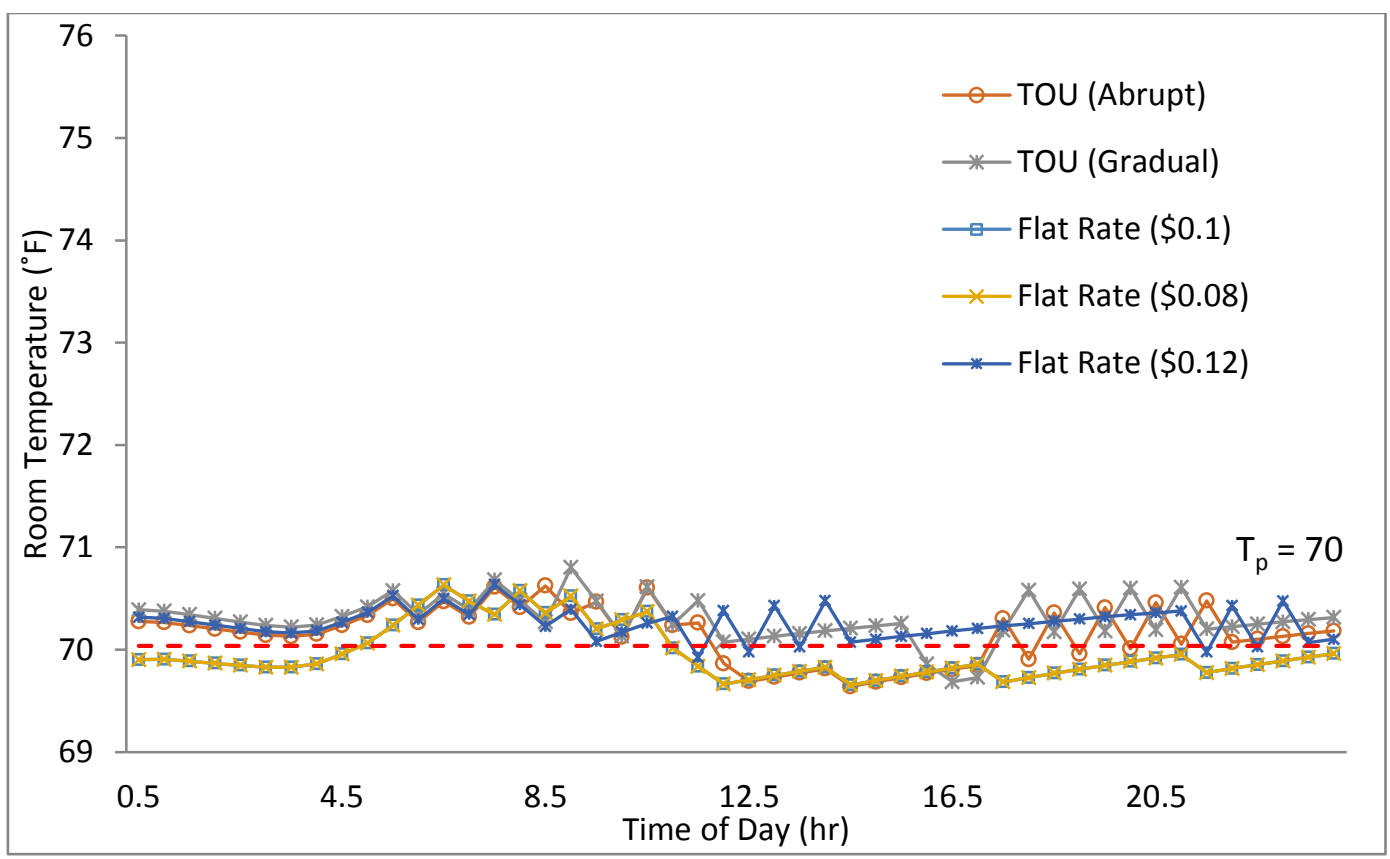

Figure 49: Temperature response for a high income (comfort-favoring) household

Next, we analyze the effect of varying risk aversion level on the response of the household. To do this, we select a household with equal weight for cost and comfort and vary $\alpha$ parameter of one attribute while keeping the other at a constant risk-neutral value. The results, summarized in Table 17, show that the average discomfort for a household decreases as $\alpha_{1}$ increases, indicating higher comfort would be experienced by those who are more risk-averse with respect to cost. The opposite is the case for $\alpha_{2}$, which when increased, increases the discomfort. This shows that the more risk-averse one becomes with respect to discomfort, the lower the discomfort he or she will experience. As for the change in cost, increase in $\alpha_{2}$ increases the cost of a household, whereas for $\alpha_{1}$ the minimum cost found at a risk-neutral value. 
Table 17: The effect of varying risk nature

\begin{tabular}{|c|c|c|c|c|c|c|c|}
\hline \multirow{2}{*}{$\boldsymbol{\alpha}_{2}=-0.01$} & \multicolumn{3}{|c|}{$\alpha_{1}$} & \multirow{2}{*}{$\alpha_{1}=-0.1$} & \multicolumn{3}{|c|}{$\alpha_{2}$} \\
\hline & -0.1 & -5 & -9 & & -0.01 & -0.2 & -0.4 \\
\hline Avg $\operatorname{cost}(\$ /$ day $)$ & 0.56 & 0.57 & 0.64 & $A v g \operatorname{cost}(\$ /$ day $)$ & 0.66 & 0.63 & 0.62 \\
\hline Avg Discomfort $\left({ }^{\circ} F\right)$ & 2.27 & 2.54 & 2.69 & Avg Discomfort $\left({ }^{\circ} F\right)$ & 2.50 & 2.47 & 2.44 \\
\hline
\end{tabular}

We then run the simulation with a population of $n=30$ households with varying risk natures as discussed in Section 3.2. The results of simulation were collected based on a run length of 30 days with weather data corresponding to July, 2013 in Glasgow, KY. Although, the warm period does not affect the response of a static model, we use a 5 day warm up period to stay consistent with the dynamic pricing model explained in the next section. As shown in Table 18, households in Group A with higher weight for cost incurred an average of $4.95^{\circ} \mathrm{F}$ discomfort at a small cost of $\$ 0.58$. On applying the TOU rates they observed an average discomfort of $4.62^{\circ} \mathrm{F}$. Group B with equal weight for cost and comfort incurred an average of $3.05^{\circ} \mathrm{F}$ discomfort at $\$ 0.72$, which changed to $2.53^{\circ} \mathrm{F}$ at $\$ 0.76$ with TOU. The reduction of the discomfort with respect to temperature deviation indicates a shift in behavior. On the contrary, group $\mathrm{C}$ experiences a small change $\left(0.02^{\circ} \mathrm{F}\right)$ in the already low average discomfort $\left(0.58^{\circ} \mathrm{F}\right)$, which is consistent with the single household result indicating negligible shift in behavior.

Table 18: Analysis of TOU vs. flat rate for $\mathrm{n}=30$ households

\begin{tabular}{|c|c|c|c|c|c|c|}
\hline \multirow[b]{2}{*}{$n=30$} & \multicolumn{3}{|c|}{ Flat Rate (\$0.1) } & \multicolumn{3}{|c|}{ TOU Rate } \\
\hline & $\begin{array}{l}\text { Avg Room } \\
\text { Temp }\left({ }^{\circ} \mathrm{F}\right)\end{array}$ & $\begin{array}{c}\text { Avg } \\
\text { Cost } \\
\text { (\$/day) }\end{array}$ & $\begin{array}{c}\text { Avg } \\
\text { Discomfort } \\
\left({ }^{\circ} \mathrm{F}\right)\end{array}$ & $\begin{array}{l}\text { Avg Room } \\
\text { Temp }\left({ }^{\circ} F\right) \\
\end{array}$ & $\begin{array}{c}\text { Avg } \\
\text { Cost } \\
\text { (\$/day) }\end{array}$ & \begin{tabular}{|c|} 
Avg \\
Discomfort \\
$\left({ }^{\circ} \mathrm{F}\right)$ \\
\end{tabular} \\
\hline Group A (cost-favoring) & 75.66 & 0.58 & 4.95 & 75.03 & 0.60 & 4.62 \\
\hline Group B (neutral) & 73.64 & 0.72 & 3.05 & 73.11 & 0.76 & 2.53 \\
\hline Group C (comfort-favoring) & 71.07 & 0.89 & 0.58 & 71.20 & 0.89 & 0.60 \\
\hline
\end{tabular}


The effect of changing energy consumption of TCL can also be examined from a different perspective as shown in Figure 50. Load factor (LF) and load variation (VAR) are used to measure the load leveling effects of the different TOU pricings. For the flat rate case, the LF was 0.68 and VAR was 3.66. The highest LF (0.71) was obtained by the TOU gradual price tariff. This load variation reduction and load factor increase indicates a better load leveling effect.

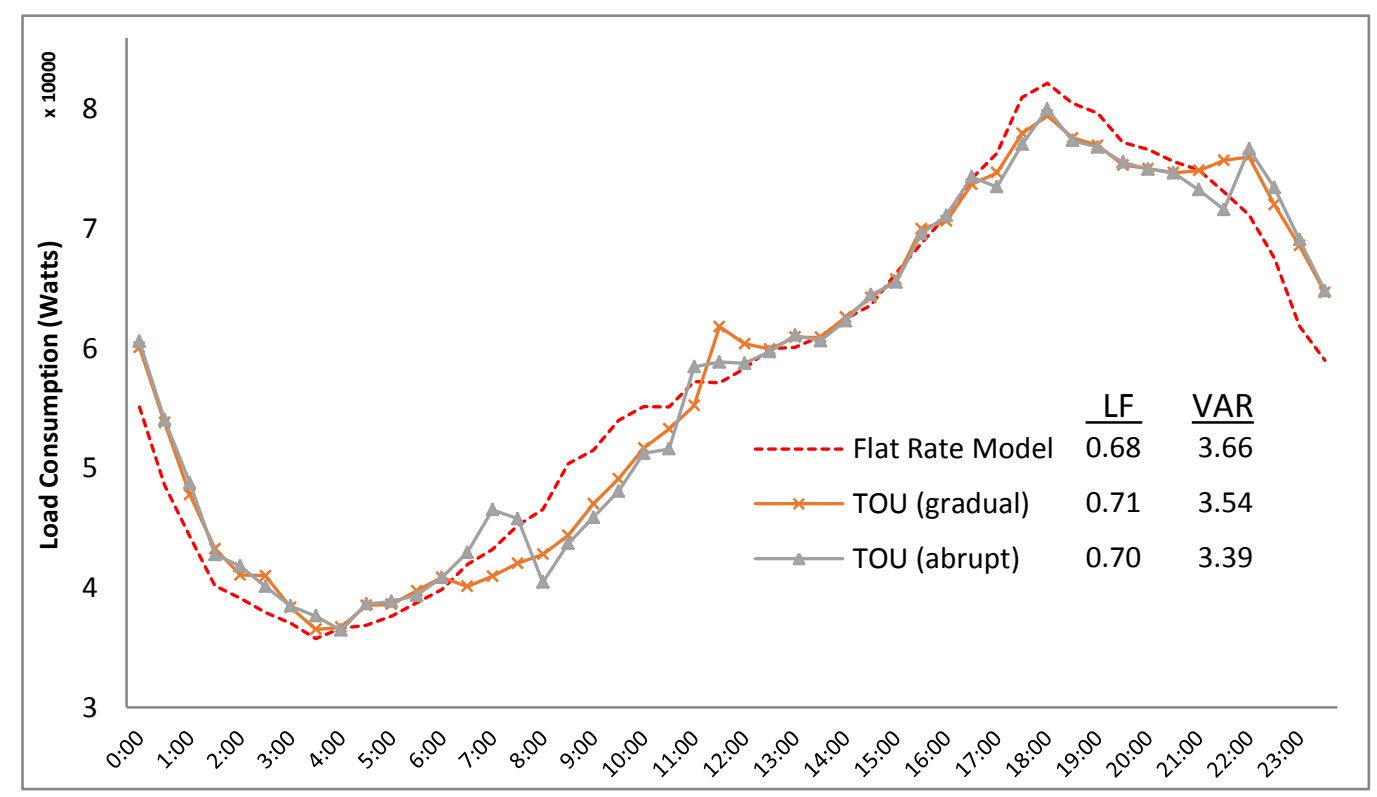

Figure 50: Average hourly load profile under different pricing structures

The next step in the analysis of static variable pricing for the TCL model includes an enriched simulation experiment. We increase the population size to $n=100$. Among the various rates described in Table 10 in Section 5.2, we assume the demand charge based on user peak, TOU and TOU with demand charge as static variable prices. The demand charge in these cases is only applied to the user"s own individual peak consumption. Since a consumer has control over his own peak consumption and the 
demand charge rates are also known in advance, we can assume that all the above mentioned pricing models are static. We use the temperature profile data from Glasgow, KY for the year 2013 as an input and run our simulation model from 6/1/2013 to 8/30/2013, excluding the 30 day warm-up period. The simulation model is robust enough to handle both heating and cooling loads, but we analyze the load shifting behavior for the summer months considering a higher impact of weather during this time for the area. Similar experiments can also be conducted for winter loads using the model. The performance measures considered are average daily load and coincident load factor. In regards to the intended system wide effect of demand response, only coincident load is of importance since it dictates the system peak level. The individual peak values are not relevant to the utility company. The average monthly bill and coincident load are also displayed as secondary measures.

A standard flat rate (FR) of $\$ 0.088 / \mathrm{kWh}$ is considered to be the baseline model to which we compare the following rates. The comparability of these pricing models based on the equivalent daily cost has already been discussed in Table 11, Section 5.2.

- UP1: flat rate of $\$ 0.076 / \mathrm{kWh}$, demand charge of $\$ 5 / \mathrm{kW}$ on user peak

- UP2: flat rate of $\$ 0.062 / \mathrm{kWh}$, demand charge of $\$ 12 / \mathrm{kW}$ on user peak

- TOU: off-peak rate of $\$ 0.052 / \mathrm{kWh}$, on-peak rate of $\$ 0.16 / \mathrm{kWh}$

- TOU-DC: standard flat rate of $\$ 0.056 / \mathrm{kWh}$, off-peak demand charge of $\$ 3.25 / \mathrm{kW}$, on-peak demand charge of $\$ 12.68 / \mathrm{kW}$

The average daily load, monthly bill, coincident load and coincident load factor for the population are shown in Figure 51 below. The red bar indicates the baseline model while the blue bars indicate demand charge rates and the yellow bar shows the TOU rates. 
Avg Daily Load (Population)

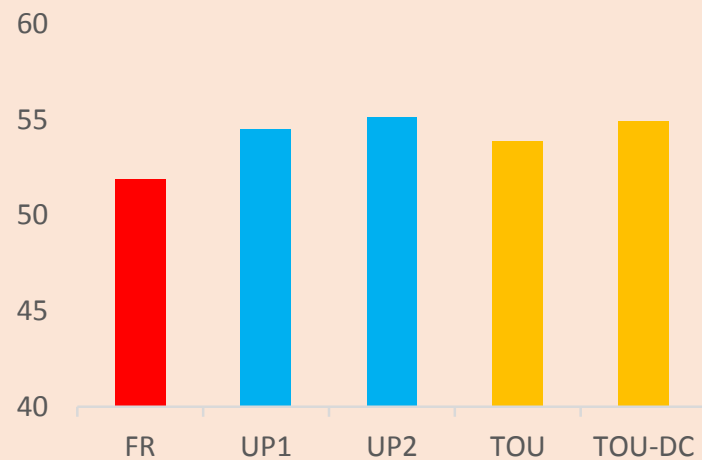

Avg Coincident Load (Population)

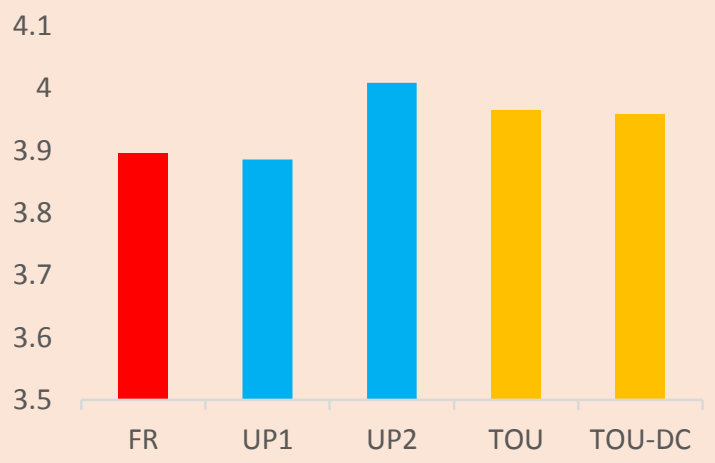

Avg Monthly Bill (Population)

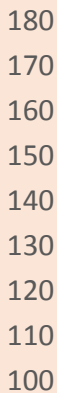

180

170

160

150

140

130

120

110

100

UP1

Avg Coincident LF (Population)

0.8

0.75

0.7

0.65

0.6

0.55

0.5

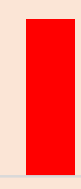

FR

UP1
UP2

TOU TOU-DC
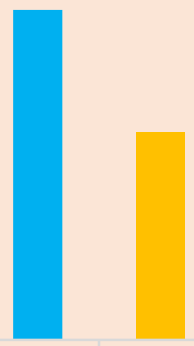

.

Figure 51: Comparison of static variable pricing models showing average daily load,

monthly bill, coincident load and coincident load factor for the population $(n=100)$

Table 19: Average monthly bill and coincident load factor (LF) for different household types under various static pricing models

\begin{tabular}{|c|c|c|c|c|c|c|c|c|c|c|}
\hline & \multicolumn{5}{|c|}{ Avg Monthly Bill (\$) } & \multicolumn{5}{c|}{ Coincident LF } \\
\cline { 2 - 11 } & $\begin{array}{c}\text { Flat } \\
\text { Rate }\end{array}$ & UP1 & UP2 & TOU & $\begin{array}{c}\text { TOU } \\
- \text { DC }\end{array}$ & $\begin{array}{c}\text { Flat } \\
\text { Rate }\end{array}$ & UP1 & UP2 & TOU & $\begin{array}{c}\text { TOU } \\
- \text { DC }\end{array}$ \\
\hline Group A & 146.26 & 151.39 & 168.8 & 143.9 & 173.68 & 0.5977 & 0.6344 & 0.6045 & 0.6387 & 0.6315 \\
\hline Group B & 146.06 & 164.41 & 173.23 & 143.59 & 177.91 & 0.6432 & 0.6288 & 0.6191 & 0.6876 & 0.6273 \\
\hline Group C & 151.2 & 163.29 & 181.83 & 154.52 & 182.1 & 0.6377 & 0.6433 & 0.6479 & 0.6737 & 0.6525 \\
\hline $\begin{array}{c}\text { Total } \\
\text { Population }\end{array}$ & 147.61 & 159.57 & 173.42 & 146.12 & 177.3 & 0.6259 & 0.6348 & 0.6203 & 0.6643 & 0.6375 \\
\hline
\end{tabular}


We can observe from Figure 51 that all four variable rates cause an increase in load consumption when compared to the flat rate. As consumers are provided with a variable pricing scheme, it is possible that they take advantage of this during the off-peak periods, thus causing an increase in average daily load consumption. However, it is important to look at the average coincident load factor among the different rates to study the effect on system peak reduction. We can see that the TOU rate performs the best in terms of increasing coincident load factor. The average monthly bill is also the lowest for the TOU rate, lower that the flat rate model. In fact, the demand charge rates actually prove to be unfavorable to the consumers, as they significantly increase the average monthly bill for the population. The marginal improvement in coincident load factor for these demand charge rates is not significant enough to offset the increase in cost. The failure to provide any significant improvement in coincident load factor by UP1 and UP2 is mainly because the demand charge is applied to the useres individual peak which may or may not coincide with the system peak.

The marginal effects of the static variable rates on different groups of households with respect to cost and contributing load factor are presented in Table 19. The varying response behaviors between different groups can be seen while comparing the monthly bill changes between flat rate and TOU. While both group A and B demonstrate a decrease in monthly bill under TOU, group $\mathrm{C}$ shows an increase. This justifies the behavior of the high-income households as defined by the scaling factors in their utility functions. The standard rate with TOU demand charge performs the worst among all the rates because the demand charge is being assessed separately during the on-peak and offpeak times. We leave the discussion of coincident load factor for the dynamic pricing 
models in the next section, since static pricing models mostly penalize only the individual peak thus failing to establish a causal relationship for analysis. As an implicit effect however, we do see that all the groups have the highest coincident load factor with the TOU model, proving its advantage among the static pricing models.

\subsection{TCL UNDER DYNAMIC PRICING}

The scope of demand response programs using variable pricing models has been mostly limited to deterministic or static methods. The adoption of dynamic pricing proves to be particularly difficult due the gross uncertainty surrounding load consumption behavior. Some utilities have adopted real-time pricing methods in various ways, but in general this area presents itself as involving high risk for the load serving entities. This is probably why most of the known real-time pricing models simply roll the spot market prices to its consumers, thus attempting to place the risk on the end-user rather than the utility provider. In this section we conduct some experiments using dynamic pricing methods on the TCL model. The assumption here is that majority of load fluctuation is caused by weather changes, specifically the outside temperature.

We first study a simple real-time pricing model based on the system energy usage and ambient temperature, as given in Equation (57) in Section 5.2. The simulation model records the real-time prices for every hour in order to generate a probability distribution which is used by the consumer while making consumption decision. A warm-up period needs to be identified in order to allow for the electricity rates to somewhat stabilize before we can collect any data. The first five days are considered as a warm-up period as we see high fluctuation of RTP rates during this period before a stable rate structure is 
obtained (see Figure 52). We will omit this period and only consider the response after the warm-up period. In contrast to the NTL model, the real time pricing (RTP) functions will retain both $p^{e}$ and $p^{t}$ coefficients in Equation (57). However, as a sensitivity analysis, we consider the following forms of the real-time prices.

- RTP: $\$ 0.1+(\$ 0.05 * \Delta e)+(\$ 0.05 * \Delta t)$

- RPT-t: $\$ 0.1+(\$ 0.1 * \Delta t)$

- $\quad$ RTP-e: $\$ 0.1+(\$ 0.1 * \Delta e)$

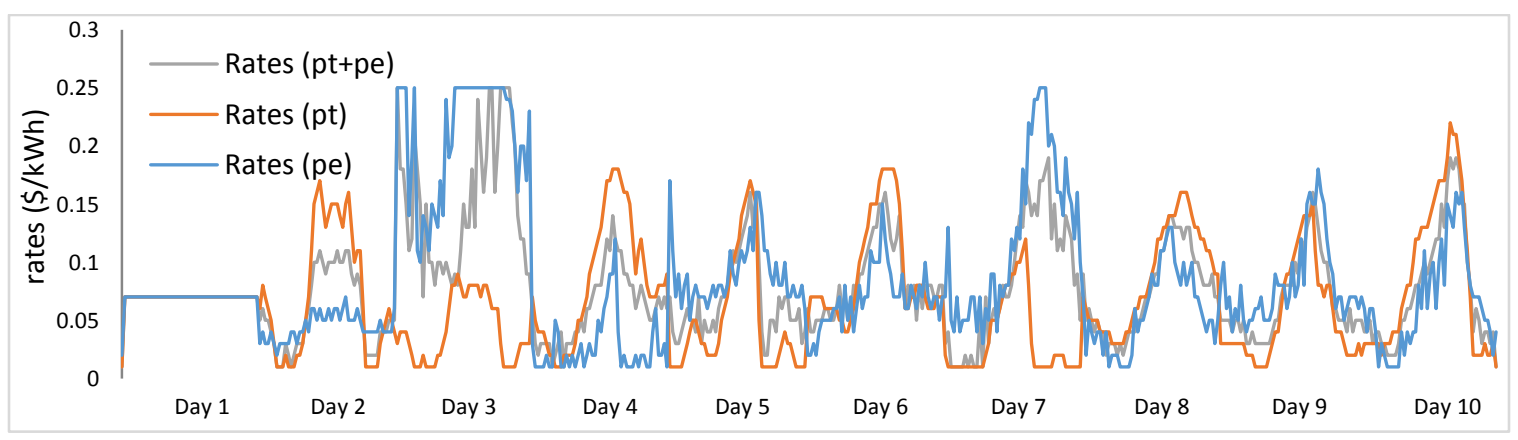

Figure 52: Simulation of rates under various RTP models for the first 10 days

Figure $53(\mathrm{a}, \mathrm{b}$ and $\mathrm{c})$ depicts the daily energy consumption of HVAC load for low-, medium- and high-income households, respectively. The characteristics of the households are evident from the different shifting behaviors in their TCL load. For example, Figure 53a shows that households belonging to Group A exhibit the most load shifting behavior, mainly due to the fact that they are highly affected by change in electricity prices. Group C on the other hand, exhibits very little change in their behavior and signifies that they are not affected by cost as much as the others (Figure 53c). The load shifting by medium income households are also not as significant. 


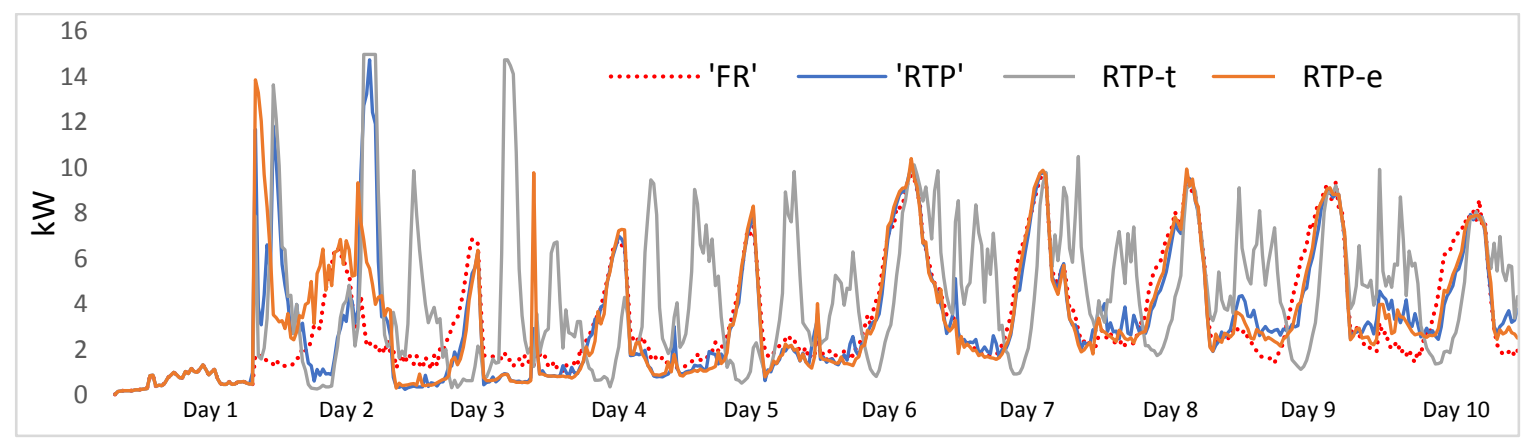

(a)

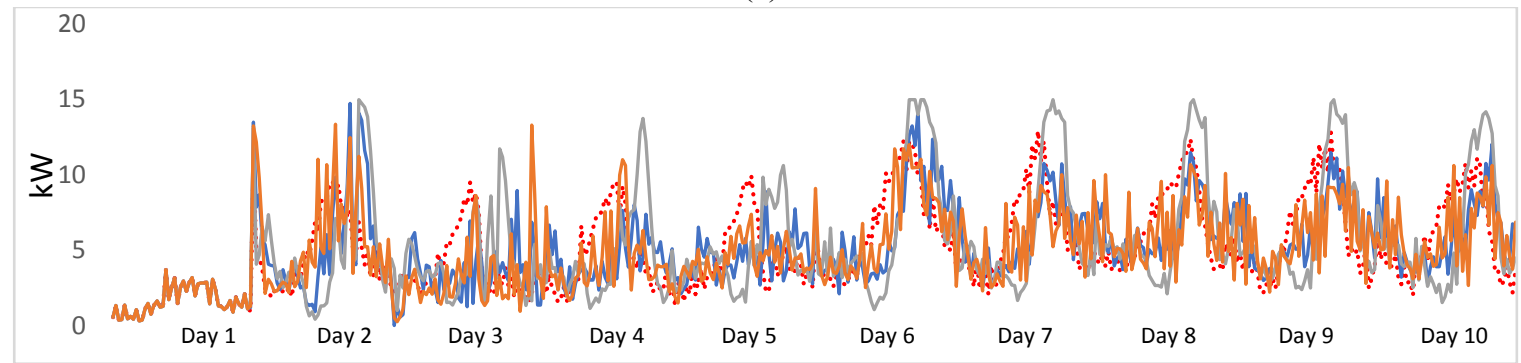

(b)

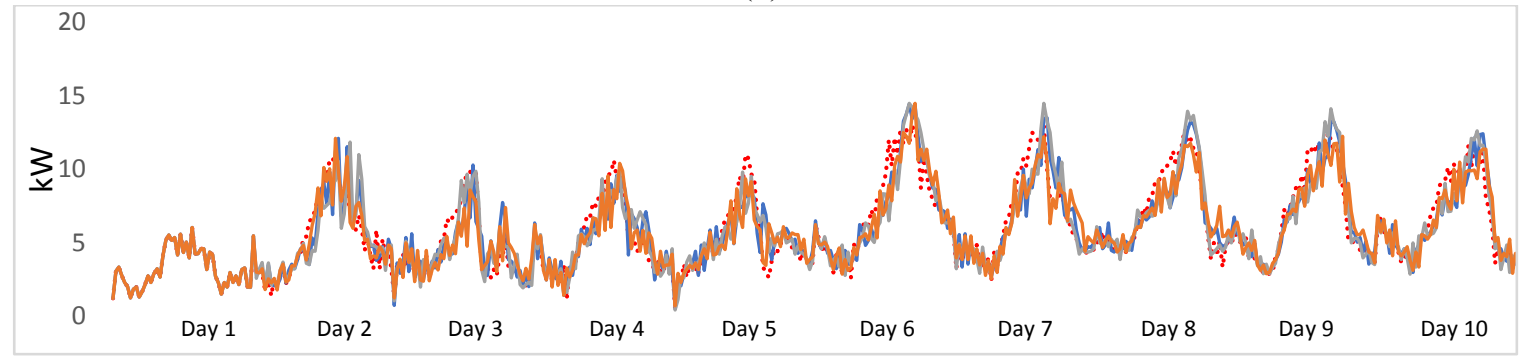

(c)

Figure 53: Energy consumption of HVAC by (a) Group A, (b) Group B and (c) Group C

Similar behavior can also be observed through the average room temperatures.

Figure 54 shows the change in the households ${ }^{\text {ee }}$ average room temperature for different price structures. We can see that Group A (Figure 54a) maintains their average room temperature farthest from their preferred temperature among the three groups in a tradeoff between cost and comfort. They experience an average discomfort of approximately $4.5^{\circ} \mathrm{C}$, while Group $\mathrm{B}$ and $\mathrm{C}$ experience only $2.5^{\circ} \mathrm{C}$ and $0.6{ }^{\circ} \mathrm{C}$ of discomfort, respectively. As seen in Figure 54c, Group C are the least likely to give up their average room temperature in return for cost benefits. In the case of RTP-t, we can see that Group 
A and B use more energy during the off-peak (when ambient temperature is lowest) in order to cool down the house nearer to their preferred temperature. This is behavior is not seen for the energy dependent RTP-e price, since the prices are affected by total system load. If the users respond to low prices during low system load, it drives the system load back up along with the price, thus negating the intended effect.

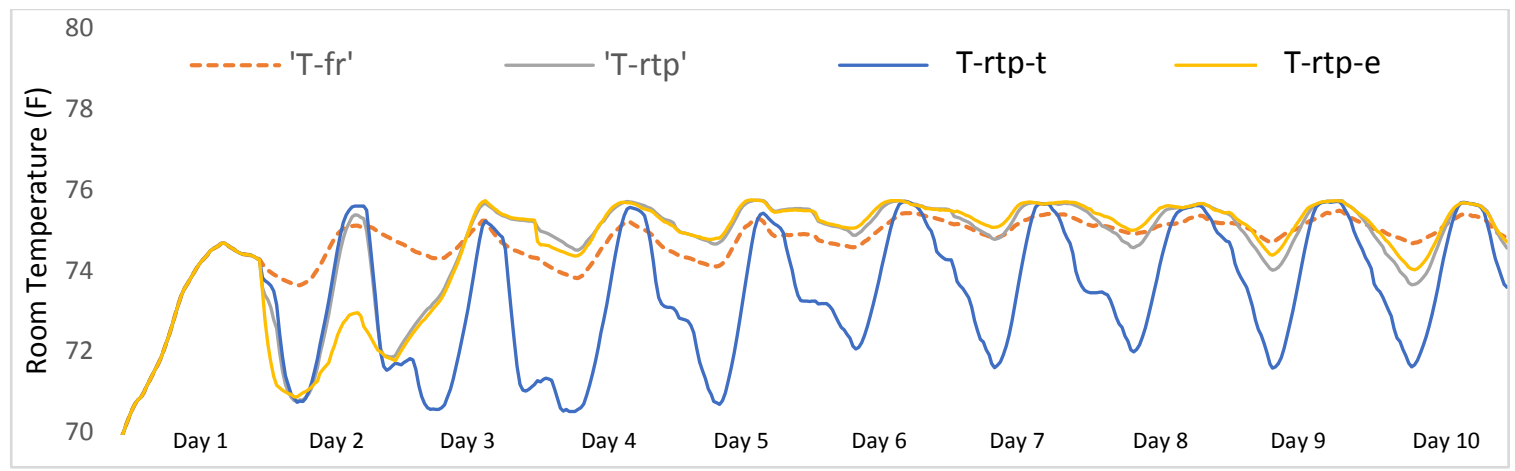

(a)

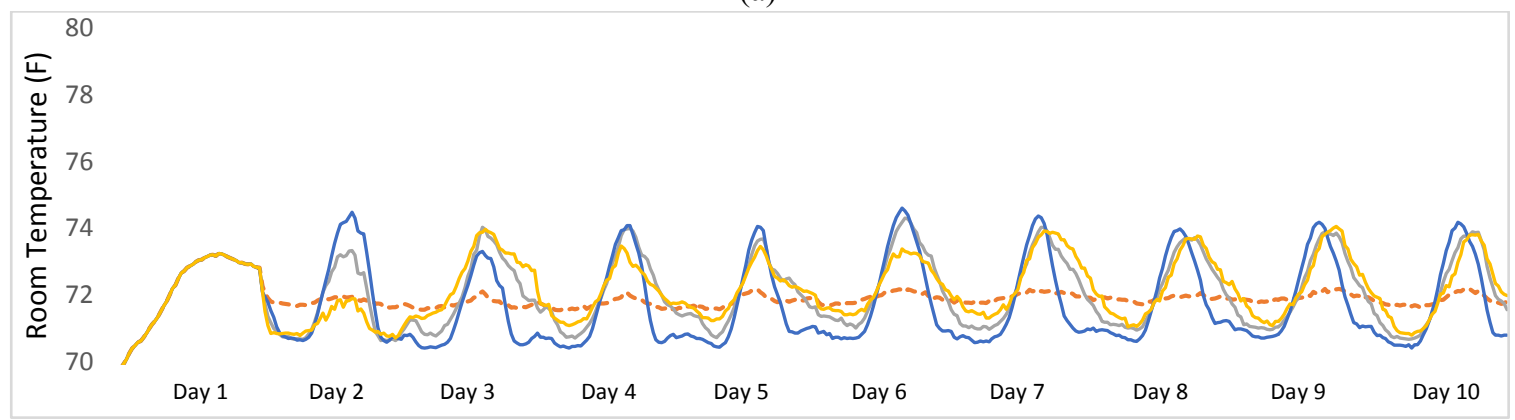

(b)

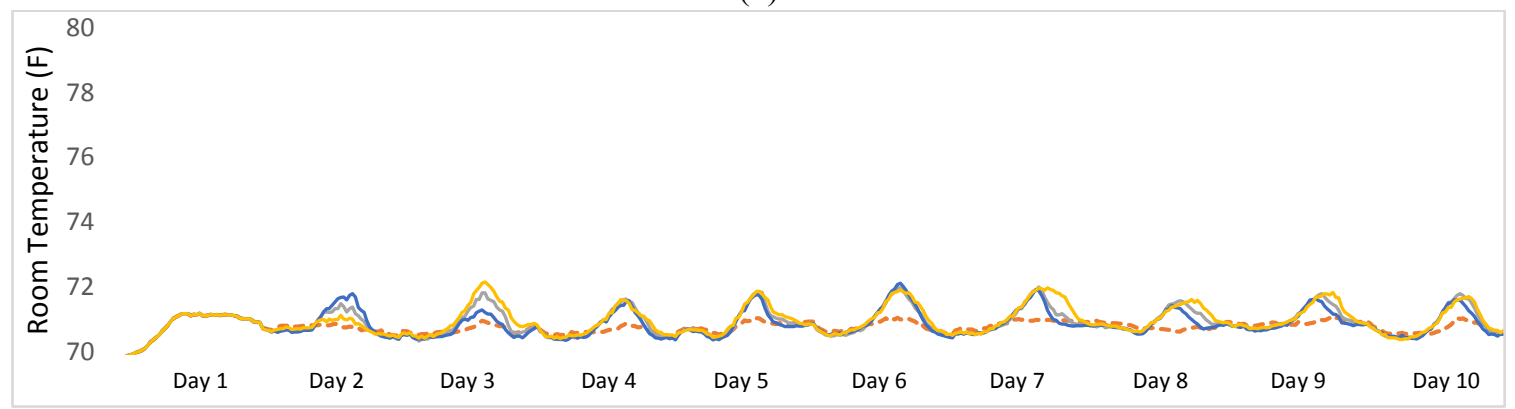

(c)

Figure 54: Average room temperatures under various pricing structures for (a) Group A, (b) Group B and (c) Group C 
We can compare the load factor (LF) and load variation (VAR) values for the different price functions in Table 20. From the table we see strong evidence that RTP-t, price dependent only on ambient temperature, does not improve these parameters but only makes it worse. We saw from the average temperature graphs above that a biased response was being observed. The population LF decreases from 0.529 to 0.473 while the VAR becomes much higher at 9.833. Similar trends are seen for individual groups also. Furthermore for all household groups, RTP and RTP-e provide improvements on both LF and VAR. The population LF increases to about 5.7 and a much lower variation (VAR $\approx 4.9)$ is obtained using either RTP or RTP-e.

Table 20: LF and VAR for TCL model under various RTP functions

\begin{tabular}{|c|c|c|c|c|c|c|c|c|}
\hline & \multicolumn{4}{|c|}{ LF } & \multicolumn{5}{c|}{ VAR } \\
\cline { 2 - 10 } & Flat Rate & $R T P$ & $R T P-t$ & $R T P-e$ & Flat Rate & $R T P$ & $R T P-t$ & $R T P-e$ \\
\hline Group A & 0.479 & 0.539 & 0.413 & 0.539 & 13.084 & 6.719 & 14.561 & 7.498 \\
\hline Group B & 0.536 & 0.512 & 0.407 & 0.523 & 8.208 & 6.522 & 16.346 & 6.614 \\
\hline Group $\boldsymbol{C}$ & 0.570 & 0.553 & 0.534 & 0.577 & 6.980 & 6.115 & 6.735 & 5.887 \\
\hline $\begin{array}{c}\text { Total } \\
\text { Population }\end{array}$ & 0.529 & 0.573 & 0.473 & 0.576 & 9.065 & 4.853 & 9.833 & 4.935 \\
\hline
\end{tabular}

The use of a novel approach for dynamic variable pricing using demand charge has been discussed in Section 5.2 earlier. We will look at some experiment results from the TCL model using rates with coincident demand charge. Unlike the demand charge applied to the user peak, coincident demand charge is applied to the energy consumed during the hour of the month at which the system was at peak. By doing so, consumers are encouraged to reduce energy consumption during potential system peak periods rather than simply reducing their own individual peak. The following rates will be used in the simulation model for this purpose. 
- CP1: flat rate of $\$ 0.076 / \mathrm{kWh}$, demand charge of $\$ 5 / \mathrm{kW}$ on coincident peak

- CP2: flat rate of $\$ 0.062 / \mathrm{kWh}$, demand charge of $\$ 12 / \mathrm{kW}$ on coincident peak

- Info: TOU energy rates $(\$ 0.12 / \mathrm{kWh}$ on-peak, $\$ 0.04 / \mathrm{kWh}$ off-peak), demand charge of $\$ 10.67 / \mathrm{kW}$ on coincident peak

$\mathrm{CP} 1$ and $\mathrm{CP} 2$ use standard flat rate structures along with a varying degree of demand charge applied to coincident peak. The Infotricity rate basically combines a TOU rate structure along with the addition of the demand charge. This rate structure is taken from the pilot study currently being tested by the Glasgow Electric Plant Board (GEPB) since January 2016.

As one can imagine, this kind of a rate system relies on the ability to accurately predict when the system will be at peak for a given month. We have discussed the method of using a conditional markov chain in order to forecast the probability of system peak in Section 4.4. The markov chain model uses historical information to predict future states of the system and thus requires a learning period or warm-up period. In order to estimate the warm-up period, we run the simulation and collect the steady state probability $(\pi 1)$ that the system will peak at every hour. We collect this information for all temperature conditions. Figure 55 shows how the steady state probability of system peak under the condition of high load stabilizes over time. Please refer to Section 4.4 for the definitions of various temperature conditions. At the beginning of the simulation $\pi 1$ is very high since there is not enough information to establish the probabilities. After collecting enough information of the state changes, a more stable probability distribution is obtained. We can see the steady state probability becomes stable after about 15 days. We use 30 days as a warm-up period just to be safe. 


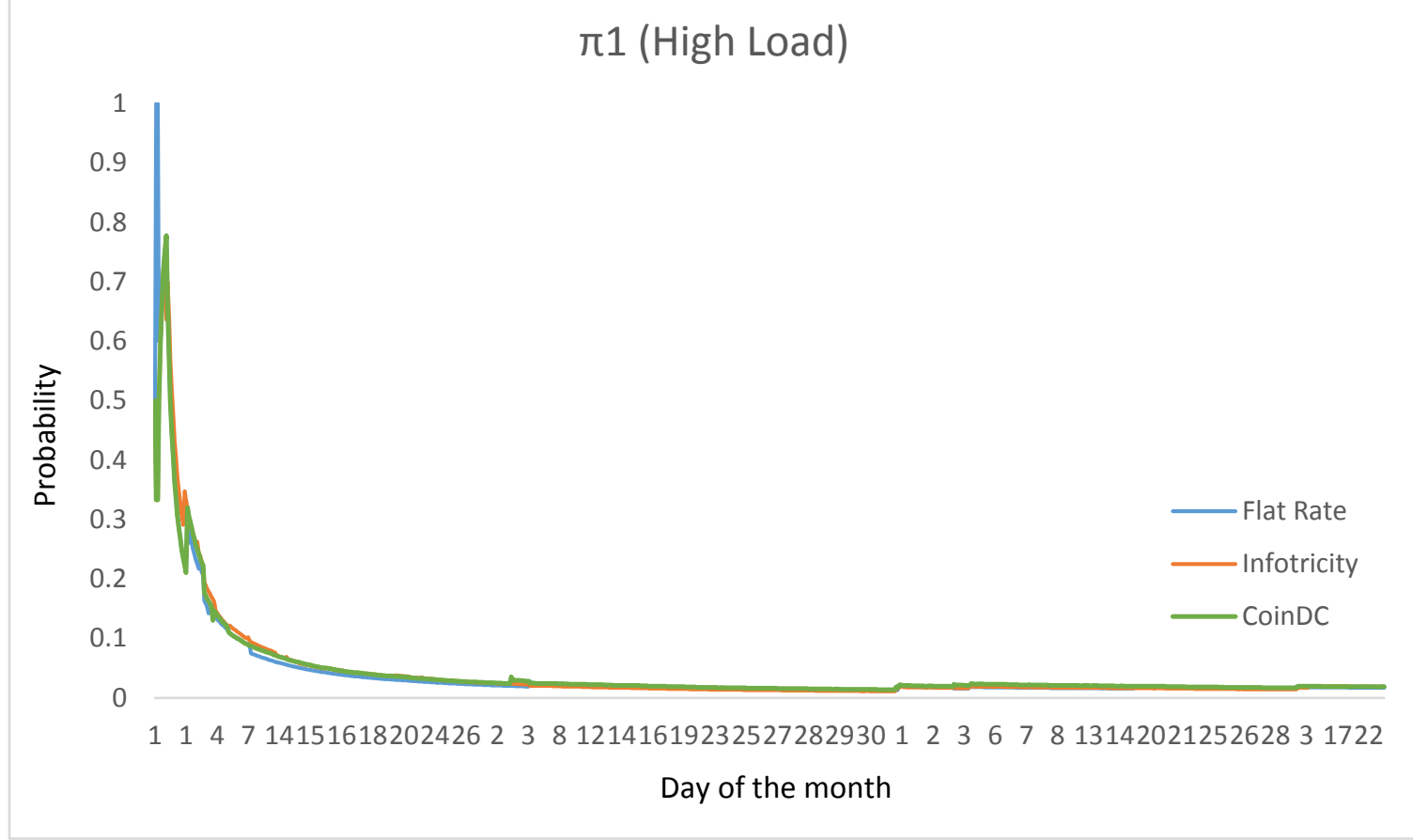

Figure 55: Steady state probability that the system will peak over time

The performances of the different pricing models using demand charge are illustrated in Figure 56, where the red bar indicates the baseline model (flat rate of $\$ 0.088 / \mathrm{kWh}$ ), the blue bars denote the demand charge models with flat rate (CP1, CP2) and the yellow bar denotes the Infotricity rate which includes TOU along with demand charge. As with any other variable pricing method, static or dynamic, we can immediately see an increase in average daily load consumption. The average monthly bills under all three pricing models are similar to each other which indicate a consistent level of demand charge. We can see from the figure that, between CP1 and CP2, the average monthly bill is similar although the average daily load is lower for CP2. This is because $\mathrm{CP} 2$ has a higher demand charge rate and thus the extra amount is the bill is a result of the higher demand charge. 


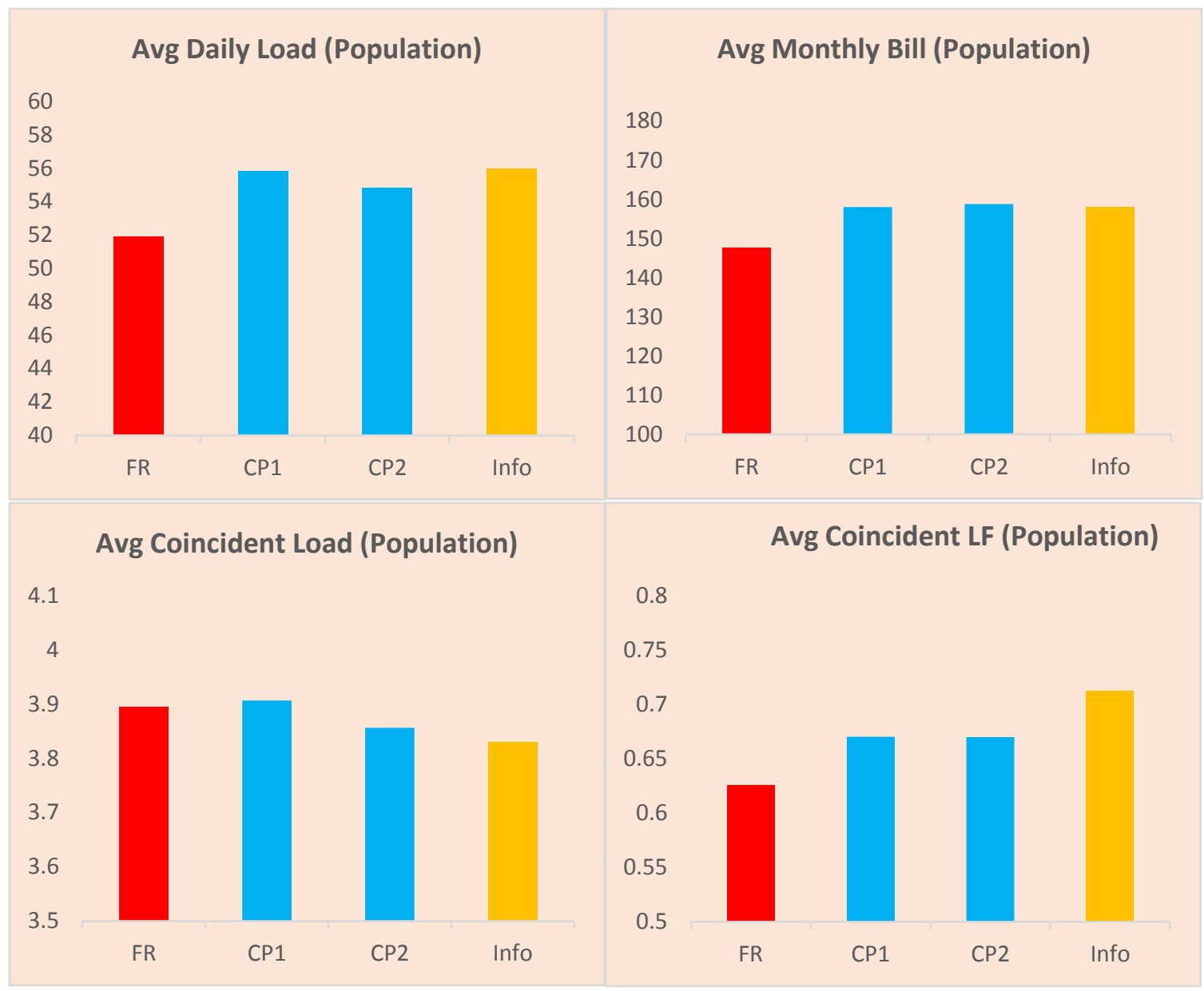

Figure 56: Comparison of the demand charge based dynamic variable pricing models showing the population response of average daily load, monthly bill, coincident load and coincident load factor $(\mathrm{n}=100)$

The Infotricity rate seems to outperform all the other rates, which is evident from the coincident load and load factor (Figure 56). A considerable increase in the coincident load factor (0.71) is indicative of a successful peak reduction. We can further examine the individual response behaviors of the different groups. The low-income households (Group A) have the highest improvement in coincident LF under the Infotricity rate, from 0.5977 to 0.7511 (Table 21), indicating the maximum reduction in load consumption 
during system peak. In contrast to this behavior, the high-income households (Group C) do not show any significant change in their coincident LF. This clearly indicates that the trade-off characteristics of comfort favoring households is not affected by cost incentive and shows no change in load behavior.

Table 21: Average monthly bill and coincident load factor (LF) for different household types under various demand charge based dynamic pricing models

\begin{tabular}{|c|c|c|c|c|c|c|c|c|}
\hline & \multicolumn{4}{|c|}{ Avg Daily Load (kWh) } & \multicolumn{4}{c|}{ Coincident LF } \\
\cline { 2 - 10 } & Flat Rate & $C P 1$ & $C P 2$ & Info & Flat Rate & $C P 1$ & $C P 2$ & Info \\
\hline Group A & 51.40 & 53.52 & 53.45 & 55.25 & 0.5977 & 0.6789 & 0.6606 & 0.7511 \\
\hline Group B & 51.32 & 57.13 & 55.18 & 56.66 & 0.6432 & 0.6792 & 0.6902 & 0.7131 \\
\hline Group C & 53.27 & 57.14 & 56.03 & 55.54 & 0.6377 & 0.6467 & 0.6440 & 0.6351 \\
\hline $\begin{array}{c}\text { Total } \\
\text { Population }\end{array}$ & 51.91 & 55.83 & 54.82 & 55.95 & 0.6259 & 0.6700 & 0.6699 & 0.7123 \\
\hline
\end{tabular}

We can also measure the performances of the various pricing models based on the steady state probabilities of the various states as defined in the markov chain model. Table 22 shows the steady state probabilities of the system being in various states under the different pricing models. A system that has a lower probability of going to peak state at any given time is definitely better. We can see from the table that the steady state probability of the system going to peak state $(\pi 1)$ is reduced by applying any of the three demand charge rates when compared to the baseline flat rate model. However, we see that the Infotricity rate has the lowest value for $\pi 1(2.22 \%)$. Figure 57 also shows the performance of the Infotricity rate based on the value of $\pi 1$. In our simulation model we consider the intermediate state as being within $20 \%$ of the current system peak. It is found that although the steady state probability of peak state is being reduced, so is the steady state probability of the off-peak state. It is observed that, by applying the demand 
charge rates, the system is more likely to be close to the peak value (within $20 \%$ ) instead of causing peak states.

The ability to model true response behavior of a typical residential population and to quantify the benefit of using different pricing models is very critical to the development of new demand response techniques. The simulation model discussed in this dissertation includes various methods to help model and predict the true behavior of consumers as close as possible. By using such a model, we are able to understand the potential system responses under various conditions, thus enabling us to recommend or test new pricing structures.

Table 22: The steady state probabilities of the system being at peak, intermediate and offpeak states under various demand charge based dynamic rates

\begin{tabular}{|l|rrrr|}
\hline & FR & CP1 & \multicolumn{1}{c|}{ CP2 } & \multicolumn{1}{c|}{ Info } \\
\hline $\boldsymbol{\pi} \mathbf{1}$ & $2.530 \%$ & $2.398 \%$ & $2.496 \%$ & $2.222 \%$ \\
\cline { 1 - 1 } $\boldsymbol{\pi}$ & $31.328 \%$ & $34.185 \%$ & $33.984 \%$ & $35.050 \%$ \\
\cline { 1 - 1 } & $66.142 \%$ & $63.417 \%$ & $63.520 \%$ & $62.728 \%$ \\
\hline
\end{tabular}

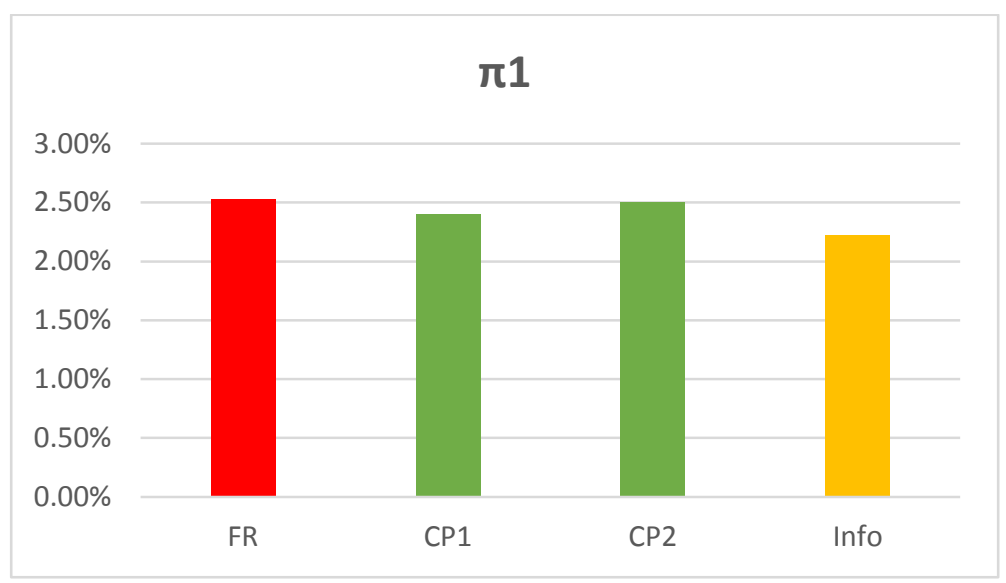

Figure 57: The steady state probabilities of the system being at peak state under various demand charge based dynamic rates 


\subsubsection{Discussions on the Effect of Residential Demand Charge}

The main purpose of this study is to understand and model the response behavior of consumers faced with a trade-off decision including a cost incentive and their own convenience, and to build a platform that helps in understanding the effect of any demand response method in a simulated environment. The responses of households are completely independent and are not controllable by the utility provider. The intended effect of demand response programs is caused not just by the direct control of loads but the collective result of an uncertain environment. High level policy decisions, like the implementation of a novel rate structure, are very unfavorable if the intended result is uncertain. That is why most demand response methods are deterministic in nature, be it in the design of the prices or the method of implementation.

The mode of load control used in demand response programs can be divided into direct and indirect load control. Some utility providers implement programs in which consumers are offered contracts where the utility company is allowed to directly control energy usage via smart meters. The utility company would then have the ability to reduce a given amount of energy by directly controlling the appliances of households to offset the system peak. This is also called a centralized control mechanism. In a smart grid environment with growing network capabilities, it makes sense for utility companies to want to use direct control methods along with variable pricing structures. By controlling the household"s energy during peak and off-peak periods, the utility company not only provides the consumer with assured cost reduction without having to keep track of pricing windows but they also mitigate the uncertainty factor. When considering direct load control, methods like pre-cooling or pre-heating are used in order to take advantage 
of the thermal capacity of the house during peak periods. This causes a forced increase in load just before the predicted peak window begins. After the peak period is over, all the houses are set back to their normal settings and the consumption spikes up because extra energy is needed to fulfill the gap. This is known as the bounce-back effect.

However, not all end users are comfortable with the idea of someone else controlling their energy consumption. Most consumers end up overriding the control action of the utility provider or not be willing to participate in direct load control altogether. Also, when a particular variable rate structure is applied to a residential network, consumers will change their consumption behavior in response to the new rate system on their own as well. The effect on system load caused by this independent behavior is not directly controllable and can be referred to as decentralized effect. In this dissertation, we assume that there is no direct load control and that all load shifting behavior is a result of decentralized behavior. It is very important to understand the decentralized effect of consumer response since this is somewhat like a gray-box for the energy providers.

From the previous section, we have concluded that the Infotricity rate has the best performance among other dynamic rates. This rate structure makes use of the TOU framework and adds a demand charge to motivate the reduction of coincident peak. Here we stress that this behavior is the expected result of a decentralized effect, and does not include direct load control. By using direct load control on consumers willing to participate in such programs, the benefit may be further increased. While using direct load control on selected participants along with a rate structure such as the Infotricity rate, it helps to understand how the independent population behaves on their own first. 
This will provide insight into how to successfully utilize direct load control. For example, we can see from our simulation results that consumers tend to simply avoid peak when the system load is approaching the incumbent peak value. This causes the system to stay close to the peak value majority of the time rather than completely shifting peak to another time period. This is called peak-shaving. With this knowledge, direct load control can simply assist in peak shifting and balance the decentralized effect of peak-shaving. 


\section{CHAPTER VI}

\section{CONCLUSION AND FUTURE WORK}

In this dissertation, we study the energy consumption scheduling behavior of enduse consumers that respond to demand response programs and various pricing schemes in a smart grid with respect to non-thermostatic loads (NTL) and thermostatic loads (TCL) using simulation. We implement multi-attribute utility functions to quantify the value of trade-off between cost incentives provided by demand response (DR) methods and the inconvenience/discomfort brought about by implementing such changes for the consumer. The consumer decision with regards to load consumption is modeled using model predictive control (MPC) mechanism, specifically in the control of HVAC loads. Further, we study an innovative demand charge for residential electricity rate and use conditional Markov chain to model the transition between peak and off-peak states for the system and generate a probability function for system peak. The simulation models thus developed are used in studying different pricing mechanisms, including existing tariffs such as flat rate pricing (FR) and time of use rates (TOU) as wells as proposed novel rate structures like real time pricing (RTP) and demand charge based rates. The simulation models are divided into two categories, NTL and TCL models, and two forms of pricing mechanisms are tested on each, one for static pricing methods and the other for dynamic rates. 
The simulation results from the NTL model show that a cost minimizing model clearly overestimates the benefits of DR methods, thus establishing the need to include the convenience factor into the study. We also conduct a design of experiment using a Latin Hypercube Design and optimize of the rate structures to minimize the PAR (1/LF) and VAR. We also show the different response patterns of consumers with varying utility functions, serving as an important observation in the process of model validation. The sensitivity analysis based on dynamic cost functions show a strong correlation between load parameters (LF, VAR) and the cost coefficients. We can see a definite improvement from a flat-rate (FR) pricing model when applying a real time price (RTP) structure, specifically for higher values of the energy-dependent price coefficient. A simple experimental design is also conducted to find optimal coefficients for RTP. In future work, this method can be used in designing optimal price functions for specific model parameters too. The key findings from the NTL model are as follows.

- A gross over-estimation of DR benefits is observed when using pricing structures that only consider a cost function as the central criteria $(31 \%$ decrease in load variance).

- Flexible NTL appliances are seen to exhibit peak-shifting behavior.

- Between an abruptly changing TOU rate plan and a gradually changing one, the gradual TOU performs marginally better. Load factor (LF) for gradual TOU is $3.7 \%$ higher and the load variance (VAR) is $3 \%$ lower than abrupt TOU rates.

- Real-time pricing (RTP) improves the performance of the model at higher values of the energy dependent coefficient $p^{e}$. 
We also study consumers ${ }^{e e}$ response to various pricings when deciding on consumption with TCLs. We conclude that the real time pricing (RTP) model using only the temperature dependent coefficient performs much poorly in comparison to the RTP model using only the energy dependent coefficient. Although one would think that a temperature dependent price would be ideal to control temperature dependent load consumption, it is found that the difference between peak and off-peak load was increased since all consumers were independently responding to weather fluctuation without any regards to the total system load. This counter-intuitive result proves to be one of the key benefits of using a detailed consumer response behavior to analyze the effects of varying cost strategies. We also propose the use of a novel pricing structure using coincident demand charge as a dynamically varying price. We observe that a TOU rate combined with coincident demand charge results in the best system performance. The key findings from the TCL model are as follows.

- TOU rates exhibit system improvement by $4.4 \%$ (gradual-TOU) and $2.9 \%$ (abrupt-TOU) on the load factor (LF); and by 3.3\% (gradual-TOU) and 7.4\% (abrupt-TOU) on variance (VAR).

- When studying coincident load factor (CLF) as a performance measure, we see that TOU rates still outperforms other static variable pricings. TOU has a $6.1 \%$ increase in CLF.

- Demand charge based on user peak causes adverse effect on CLF, as we see a decrease of about $1 \%$ when using a high demand charge $(\$ 12 / \mathrm{kW})$. 
- Standard rate with a TOU demand charge performs slightly better when compared to flat rate ( $1.8 \%$ increase in CLF) but increase the monthly average bill by almost $20 \%$, thus is not favorable from the consumer"s perspective.

- Real time pricing model dependent on only energy (RTP-e) has the highest LF when compared to flat rate ( $8.9 \%$ higher). The temperature dependent pricing (RTP-t) performs worse than the baseline (10.6\% decrease in LF).

- Among the coincident demand charge rate structures the Infotricity rate (standard TOU with coincident demand charge) performs the best.

- The standard coincident demand charge rates (CP1 and CP2) show an increase of approximately $7 \%$ in CLF, while the Infotricity shows an increase of $13.8 \%$ in CLF from the baseline flat rate model.

- The steady state probability that the system will peak $(\pi 1)$ is also found to be the lowest for Infotricity rate $(2.22 \%)$ as compared to the flat rate model $(2.53 \%)$.

Having developed the simulation models for TCL and NTL load consumption, it is important to take into account the rest of the unaccounted residential loads in addition to the simulated loads. In our analysis for different scenarios, we have considered the output parameters in the context of the simulated load by itself or by adding the rest of the unaccounted load by using a top down method. In the top down method, we simply assumed a typical load residential profile that we would expect from a population and add it to the analysis by taking the difference between the expected total load and the simulated appliance load. Even though, this allows us to observe the load behavior in a 
fuller context, we are compromising on the variability of this load by considering the remaining load to be constant. The remaining load profile, other than HVAC, washer, dryer and dishwasher, also needs to be simulated from a bottom up approach if we are to accurately simulate total load consumption. We can achieve this by modeling individual appliances separately and providing some variability in theses loads. In future studies, this can help in modeling the total residential load profile more accurately.

For future research it will also be highly beneficial to build a single model that combines the NTL and TCL model together so that pricing models may be studied as a total system response rather that different appliances separately. By doing so, we will essentially integrate the load modeling algorithms for TCL and NTL. The interaction between TCL and NTL loads can also be studied as a part of a complete load system. For example, we currently consider that ambient temperature does not have any effect on the consumption of NTL loads and we observe it in isolation. However, in reality the cost function, if affected by ambient temperature, will indirectly affect the usage of NTL as well. Also an agent based simulation approach may be used to model the interaction of different households with each other in a community behavior.

A major step in the proposed future work is the development of a macro-level MPC model to optimize real-time prices. A dual MPC method can be used where the current simulation model consisting of the micro-MPCes for individual households and macro-MPC for the utility provider system wide. Similar to the behavioral model as described in our current simulation of individual households, by forecasting ambient temperature during the prediction horizon, the utility company will try to optimize the pricing by using the simulation model as a system model (Figure 58). 


\section{System simulation model}

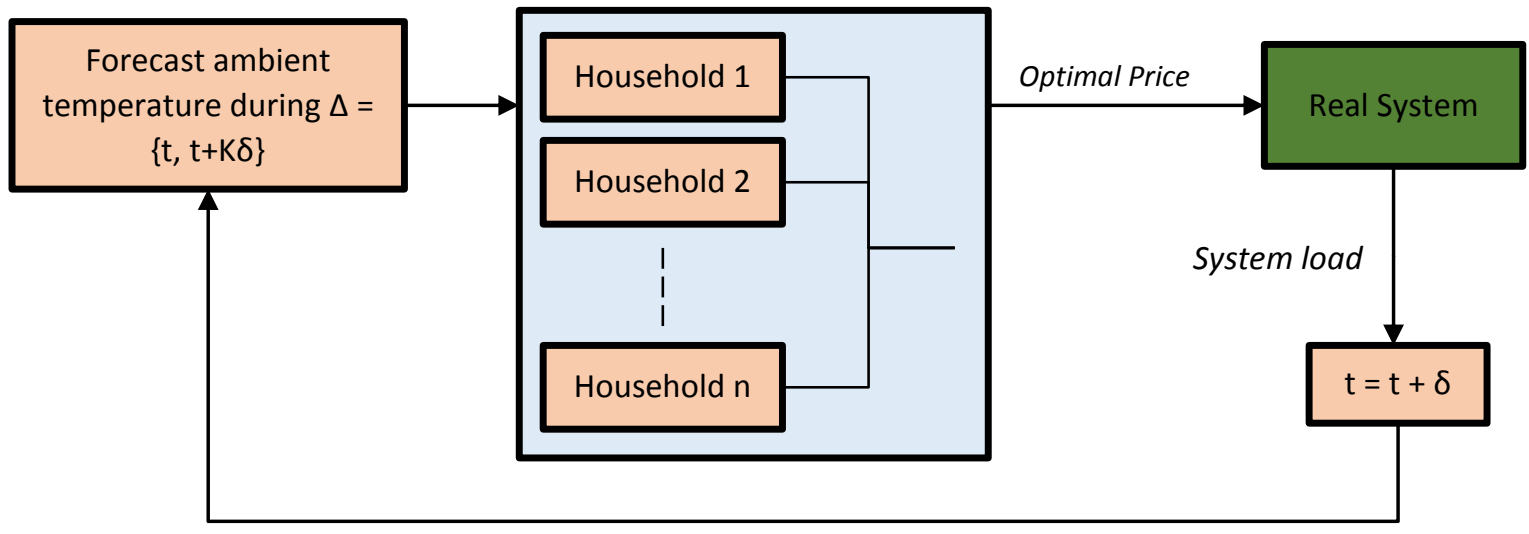

Figure 58: Macro MPC for optimization of real-time price

The objective function for this model will be as shown in Equation (62), where we are trying to minimize the difference between a desired reference load $r_{i}$ and the actual load $y_{i}$, as well as frequent change of unit price $p_{i}$ (Figure 59). $W_{1}$ and $W_{2}$ are weights given to the difference from the reference load and the change in unit price between times, respectively. This general model can then be tailored to fit various static and dynamic pricing functions accordingly and use it to determine optimal pricing strategies using the learning behavior from historical data of population response patterns. The accurate modeling of consumer response will thus be critical in the development of optimal demand response methods.

$$
\text { Minimize } J=\sum_{i} w_{1}\left(r_{i}-y_{i}\right)^{2}+\sum_{i} w_{2} \Delta p_{i}^{2}
$$




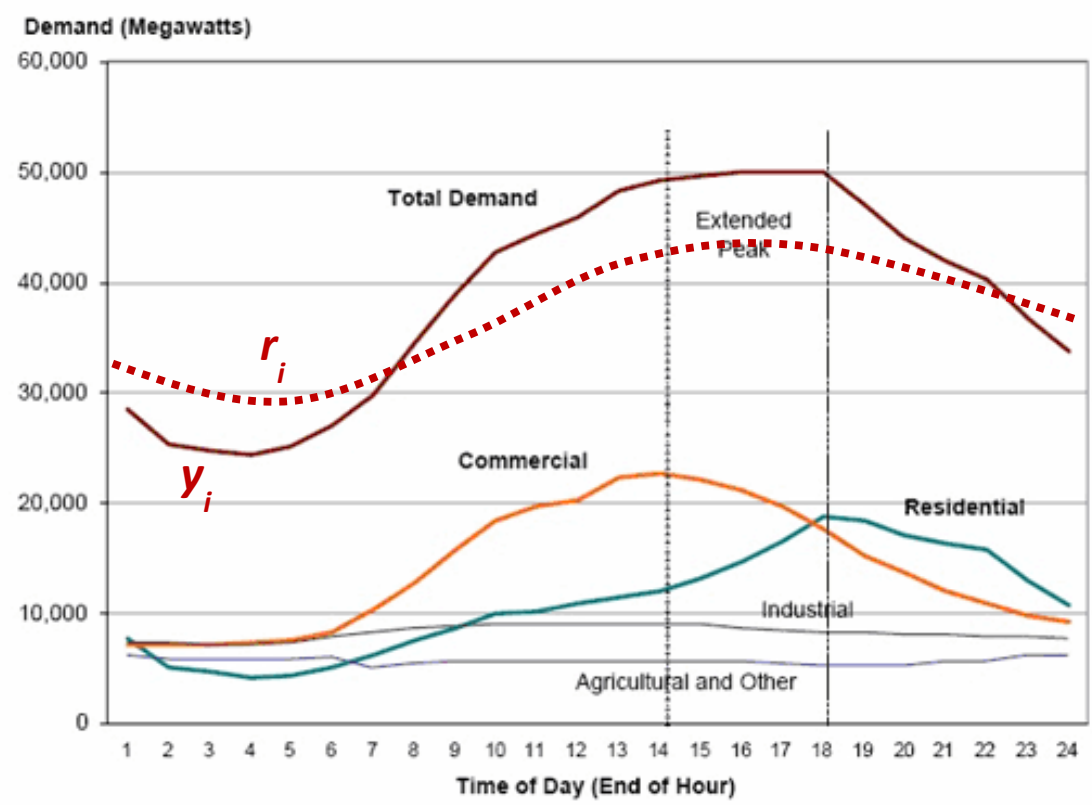

Figure 59: Reference load matching via macro-MPC model 


\section{REFERENCES}

1. Avci, M., Erkoc, M., Rahmani, A. \& Asfour, S., 2013, "Model predictive HVAC load control in buildings using real-time electricity pricing," Energy and Buildings, 60, $199-209$.

2. Azar, E. \& Menassa, C. C., 2011, “A decision framework for energy use reduction initiatives in commercial buildings," Proceedings of the 2011 Winter Simulation Conference, 816-827.

3. Bakker, V., Molderink, A., Bosman, M. G. C., Hurink, J. L. \& Smit, G. J. M, 2010, "On simulating the effect on the energy efficiency of smart grid technologies," Proceedings of the 2010 Winter Simulation Conference, 393-404.

4. Balan, R., Stan, S. \& Lapusan, C., 2009, "A Model Based Predictive Control Algorithm for Building Temperature Control," Digital Ecosystems and Technologies, $540-545$.

5. Balan, R., Hancu, O., Stan, S., Lapusan, C. \& Donca, R., 2009, “Application of a model based predictive control algorithm for building temperature control," Proceedings of 3rd WSEAS International Conference on Energy Planning, Energy Saving, Environmental Education (EPESE '09), 97-101.

6. Balan, R., Donca, R., Balan, A., Plesa, A., Pacucar, L. \& Muresan, V., 2011, "Thermal modeling and temperature control of a house," The Romanian Review Precision Mechanics, Optics and Mechatronics, 39, 59-62. 
7. Balan, R., Cooper, J., Chao, K., Stan, S. \& Donca, R., 2011, "Parameter identification and model based predictive control of temperature inside a house," Energy and Buildings, 43, 748-758.

8. Barros, G., 2010, "Herbert A. Simon and the concept of rationality: Boundaries and procedures," Brazilian Journal of Political Economy, 30, 3(119), 455-472.

9. Bodily, S. E., 1980, “Analysis of risks to life and limb," Operations Research, Design Analysis Special Issue, 28(1), 156-175.

10. Braun, J. E., 2003, "Load control using building thermal mass," Transactions of the ASME, 125, $292-301$.

11. Brounen, D., Kok, N. \& Quigley, J. M., 2013, "Energy literacy, awareness, and conservation behavior of residential households," Energy Economics, 38, 42-50.

12. Castilla, M., Alvarez, J. D., Berenguel, M., Rodriguez, F., Guzman, J. L. \& Perez, M., 2011, “A Comparison of Thermal Comfort Predictive Control Strategies," Energy and Buildings, 43, $2737-2746$.

13. Celebi, M. \& Fuller, D. J., 2007, “A model for efficient consumer pricing schemes in electricity markets," IEEE Transactions on Power Systems, 22(1), 60-67.

14. Chen, J., Taylor, J. E. \& Wei, H. H., 2011, “Toward a building occupant network agent-based model to simulate peer induced energy conservation behavior," Proceedings of the 2011 Winter Simulation Conference, 883-890.

15. Chen, L., Li, N., Low, S. H. \& Doyle, J. C., 2010, “Two market models for demand response in power networks," IEEE Smart Grid Comm, 10, 397-402.

16. Clemen, R. T. \& Reilly, T., 1996, "Making hard decisions with decision tools," Duxbury Press, Pacific Grove, CA. 
17. Collins, M. \& Mader, G. H., 1983, "The timing of EV recharging and its effect on utilities," IEEE Transactions on Vehicular Technology, VT-30(1), 90-97.

18. Commonwealth of Kentucky, 2011, "Responsive pricing and smart metering pilot program annual report for Louisville Gas and Electric Company," Kentucky Public Service Commission, Case No. 2007-00117.

19. Daryanian, B., Bohn, R. E. \& Tabors, R. D., 1989, “Optimal demand-side response to electricity spot prices for storage-type customers," IEEE Transactions on Power Systems, 4(3), 897-903.

20. Delquie, P. \& Luo, M., 1997, “A simple trade-off condition for additive multiattribute utility," Journal of Multi Criteria Decision Analysis, 6, 248-252.

21. EPRI 2012 Technical Report, "Understanding electric utility customers - summary report: What we know and what we need to know," Palo Alto, CA: 2012.1025856.

22. Exarchakos, L., Leach, M. \& Exarchakos, G., 2009, "Modeling electricity storage systems management under the influence of demand-side management programmes," International Journal of Energy Research, 33, 62-76.

23. Fahrioglu, M., \& Alvarado, F. L., 1999, "Designing cost effective demand management contracts using game theory," Proceedings of the IEEE PES Winter Meeting, New York City.

24. Fahrioglu, M., \& Alvarado, F. L., 2000, "Designing incentive compatible contracts for effective demand management," Power Systems, IEEE Transactions, 15(4), 1255 1260. 
25. Fahrioglu, M., \& Alvarado, F. L., 2001, "Using utility information to calibrate customer demand management behavior models," IEEE Transactions on Power Systems, 16(2), 317-323.

26. Goyal, S., Liao, C. \& Barooah, P., 2011, "Identification of multi-zone building thermal interaction model from data," $50^{\text {th }}$ IEEE Conference on Decision and Control, 181-186.

27. Guttromson, R. T., Chassin, D. P. \& Widergren, S. E., 2003, "Residential energy resource models for distribution feeder simulation," Power Engineering Society General Meeting, IEEE, 1, 108-113.

28. Huang, W. \& Lam, H. N., 1997, "Using genetic algorithms to optimize controller parameters for HVAC systems," Energy and Buildings, 26, 277-282.

29. Ihara, S. \& Schweppe, F., 1981, "Physically based modeling of cold load pickup," IEEE Transactions on Power Apparatus and Systems, PAS-100(9), 4142-4150.

30. Jiang, L. \& Low, S. H., 2011, "Multi-period optimal procurement and demand responses in the presence of uncertain supply," Technical Report, Caltech.

31. Katimapula, S. \& Lu, N., 2006, "Evaluation of residential HVAC control strategies for demand response programs," ASHRAE Transactions, 112(1), 535-546.

32. Kazemi, A., Modarres, M., Mehregan, M. R., Neshat, N. \& Foroughi. A., 2011, “A markov chain grey forecasting model: A case study of energy demand of industry sector in Iran," 3rd International Conference on Information and Financial Engineering, 12, 13-18.

33. Keeney, R. L. \& Raiffa, H., 1993, "Decisions with Multiple Objectives: Preferences and Value Tradeoffs," 2nd Edition, Cambridge University Press. 
34. Kelton, W. D., Smith, J. S., Sturrock, D. T., \& Verbraeck, A., 2010, "Simio \& Simulation: Modeling, Analysis, Application," McGraw Hill.

35. Kondoh. J., 2009 "A direct load management scheme to control appliances and EVs while considering end users comfort" Proceedings of Grid-Interop, Denver.

36. Krysiak, F. C. \& Weigt, H., 2015, "The demand side in economic models of energy markets: the challenge of representing consumer behavior," Frontiers in Energy Research, 3(24), 1-10.

37. Kulvanitchaiyanunt, A., Chen, V. \& Rosenberger, J., 2013, "Control for a system of PHEV charging station," Proceedings of the 2013 Industrial and Systems Engineering Research Conference, 356-366.

38. Kundu, S., Sinitsyn, N., Backhaus, S. \& Hiskens, I., 2011, "Modelling and control of thermostatically controlled loads," $17^{\text {th }}$ Power Systems Computation Conference.

39. Li, M., Feng, H., Chen, F. \& Kou, J., 2013, "Optimal versioning strategy for information products with behavior-based utility function of heterogeneous customers," Computers \& Operations Research, 40, 2374-2386.

40. Li, N., Chen, C. \& Low, H., 2011, "Optimal demand response based on utility maximization in power networks," Power and Energy Society General Meeting, 1-8.

41. Louisville Gas and Electric Company, 2013. Rate, Terms and Conditions for Furnishing Electric Service. Public Service Commission of Kentucky. Issued by Authority of an Order of the Public Service Commission in Case No. 2012-00222.

42. Lu, N. \& Chassin, D. P., 2004, "A state-queuing model of thermostatically controlled appliances," IEEE Transactions on Power Systems, 19(3), 1666-1673. 
43. Lu, N., Chassin, D. P. \& Widergren, S. E., 2005, "Modeling uncertainties in aggregated thermostatically controlled loads using a state queuing model," IEEE Transactions on Power Systems, 20, 725-734.

44. Maurer, L. T. A. \& Barroso, L. A., 2011, "Electricity auctions: An overview of efficient practices," World Bank Study, Washington, DC.

45. McIntryre, M., Burke, W.J., Latham, J. \& Graham, J., 2014, "Energy usage patterns in fielded smart appliances," 29th International Conference on Computers and Their Applications.

46. Miyamoto, J. M. \& Wakker, P., 1995, "Multiattribute utility theory without expected utility foundations," Operations Research, 44(2), 313-326.

47. Mohsenian-Rad, A. H. \& Leon-Garcia, A., 2010, "Optimal residential load control with price prediction in real-time electricity pricing environments," IEEE Transactions on Smart Grid, 1(2), 120-133.

48. Mohsenian-Rad, A. H., Wong, V. W., Jatskevich, J., \& Schober, R., 2010, “Optimal and autonomous incentive-based energy consumption scheduling algorithm for smart grid," Innovative Smart Grid Technologies (ISGT), IEEE Transactions, 1-6.

49. Molderink, A., Bosman, M. G. C., Bakker, V., Hurink, J. L. \& Smit, G. J. M, 2009, "Simulating the effect on the energy efficiency of smart grid technologies," Proceedings of the 2009 Winter Simulation Conference, 1530-1541.

50. Mortensen, R. E. \& Haggerty, K. P., 1988, “A stochastic computer model for heating and cooling loads," IEEE Transactions on Power Systems, 3(3), 1213-1219.

51. NAHB Research Center, Inc., 2001, Review of Residential Electrical Energy Use Data. PATH (Partnership for Advancing Technology in Housing). 
52. Nesbakken, R., 1999, "Price sensitivity of residential energy consumption in Norway," Energy Economics, 21, 493-515.

53. Nikovski, D., Xu, J. \& Nonaka, M., 2013, “A method for computing optimal set-point schedules for HVAC systems," Proceedings of the 11th REHVA World Congress, CLIMA 2013.

54. Pedrasa, M. A. A., Spooner, T. D. \& MacGill, I. F., 2010, “Coordinated scheduling of residential distributed energy resources to optimize smart home energy services," IEEE Transactions on Smart Grid, 1(2), 134-143.

55. Price, P. N., 2010, "Methods for analyzing electric load shape and its variability," California Energy Commission.

56. Rahimi, F. \& Ipakchi, A., 2010, "Demand response as a market resource under the smart grid paradigm," IEEE Transactions on Smart Grid, 1(1), 82-88.

57. Ramanathan, B. \& Vittal, V., 2008, “A framework for evaluation of advanced direct load control with minimum disruption," IEEE Transactions on Power Systems, 23(4), 1681-1688.

58. Ren, X., Liu, Q. \& Zhang, Y., 2015, “The proportion of energy consumption structure prediction based on markov chain," Journal of Chemical and Pharmaceutical Research, 7(3), 378-382.

59. Samadi, P., Mohsenian-Rad, A., Schober, R., Wong, V., \& Jatskevich, J., 2010, "Optimal real-time pricing algorithm based on utility maximization for smart grid," The University of British Columbia.

60. Schuwirth, N., Reichert, P. \& Lienert, J., 2012, "Methodological aspects of multicriteria decision analysis for policy support: A case study on pharmaceutical removal 
from hospital wastewater," European Journal of Operational Research, 220, 472483.

61. Sensfu, F., Ragwitz, M., Genoese, M., \& Möst, D., 2007, “Agent-based simulation of electricity markets: a literature review," Energy Studies Review, 15(2), Article 2.

62. Shimoda, Y., Fujii, T., Morikawa, T. \& Mizuno, M., 2004, "Residential end-use energy simulation at city scale," Building and Environment, 39, 959-967.

63. Shimoda, Y., Asahi, T., Taniguchi, A. \& Mizuno, M., 2007, "Evaluation of city-scale impact of residential energy conservation measures using the detailed end-use simulation model," Energy, 32, 1617-1633.

64. Simon, H., 1955, “A behavioral model of rational choice," The Quarterly Journal of Economics, 69(1), 99-118.

65. Sortomme, E., Hindi, M. M., MacPherson, S. D. J. \& Venkata, S. S., 2011, "Coordinated charging of plug-in hybrid electric vehicles to minimize distribution system losses," IEEE Transactions on Smart Grid, 2(1), 198-205.

66. Steeger, G., Barroso, L. A. \& Rebennack, S., 2013, “Optimal bidding strategies for hydro-electric producers: a literature survey," IEEE Transactions on Power Systems, 29(4), 1758-1766.

67. Stokke, A. V., Doorman, G. L. \& Ericson, T., 2009, “An analysis of a demand charge electricity grid tariff in the residential sector," Statistics Norway, Research Department, Discussion Papers No. 574.

68. Swan, L. \& Ugursal, I., "Modeling of end-use energy consumption in the residential sector: A review of modeling techniques," Renewable and Sustainable Energy Reviews, 13, 1819-1835. 
69. Thurston, Deborah L. "Multi-attribute Utility Analysis of Conflicting Preferences." Decision Making in Engineering Design. Ed. Kemper E. Lewis, et al. New York, New York: ASME Press, 2006. 125-133.

70. Marija Trčka, M. \& Hensen, J. L. M., 2010, "Overview of HVAC system simulation," Automation in Construction, 19, 93-99.

71. U. S. Census Bureau, 2010 Census Summary File. Retrieved from http://www.census.gov/2010census/.

72. U. S. Department of Energy, 2006, "Benefits of demand response in electricity markets and recommendations for achieving them," A Report to the United States Congress Pursuant to Section 1252 of the Energy Policy Act of 2005.

73. U. S. Energy Information Administration, Annual Energy Outlook 2012. DOE/EIA0383.

74. Vasak, M., Starcic, A. \& Martincevic, A., 2011, "Model predictive control of heating and cooling in a family house," MIPRO, 2011 Proceedings of the 34th International Convention, $739-743$.

75. Wallenius, J., Dyer, J. S., Fishburn, P. C., Steuer, R. E., Zionts, S. \& Deb, K., 2008, "Multiple criteria decision making, multiattribute utility theory: Recent accomplishments and what lies ahead," Management Science, 54(7), 1336-1349.

76. Wen, Y. \& Burke, W., 2013, "Real-time dynamic house thermal model identification for predicting HVAC energy consumption," IEEE Green Technologies Conference, 367-372. 
77. Wijaya, T. K., Sinn, M. \& Chen, B., 2015, "Forecasting uncertainty in electricity demand," Computational Sustainability: Papers from the 2015 AAAI Workshop, 120126.

78. Wilson, C. \& Dowlatabadi, H., 2007, "Models of Decision Making and Residential Energy Use,” The Annual Review of Environment and Resources, 32, 169 - 203.

79. Wood, G., \& Newborough, M., 2003, "Dynamic energy-consumption indicators for domestic appliances: environment, behavior and design," Energy and Buildings, 35(8), 821-841.

80. Xu, L. \& Deng, S. J., 2013, "Incentive Compatible Contracting of Thermostats Control for Renewable Energy Integration," Industrial and Systems Engineering Research Conference, 346-355.

81. Yang, P., Tang, G. \& Nehorai, A., 2013, "A game-theoretic approach for optimal Time-of-Use electricity pricing," IEEE Transactions on Power Systems, 28(2), 884892.

82. Zhou, Z., Chan, W. K. V., Chow, J. H., \& Kotsan, S., 2009, "Duopoly electricity markets with accurate and inaccurate market goals," Proceedings of the 2009 Winter Simulation Conference (WSC), 1569-1580.

83. Zhou, Z., Zhao, F. \& Wang, J., 2011, “Agent-Based Electricity Market Simulation with Demand Response from Commercial Buildings." IEEE Transactions on Smart Grid, 2(4), $580-588$. 
APPENDIX A

Utility functions for different household types showing average cost $(X)$ and average comfort $(\mathrm{Y})$ values under various pricing structures 


\section{$\underline{\text { Standard Flat Rate }}$}

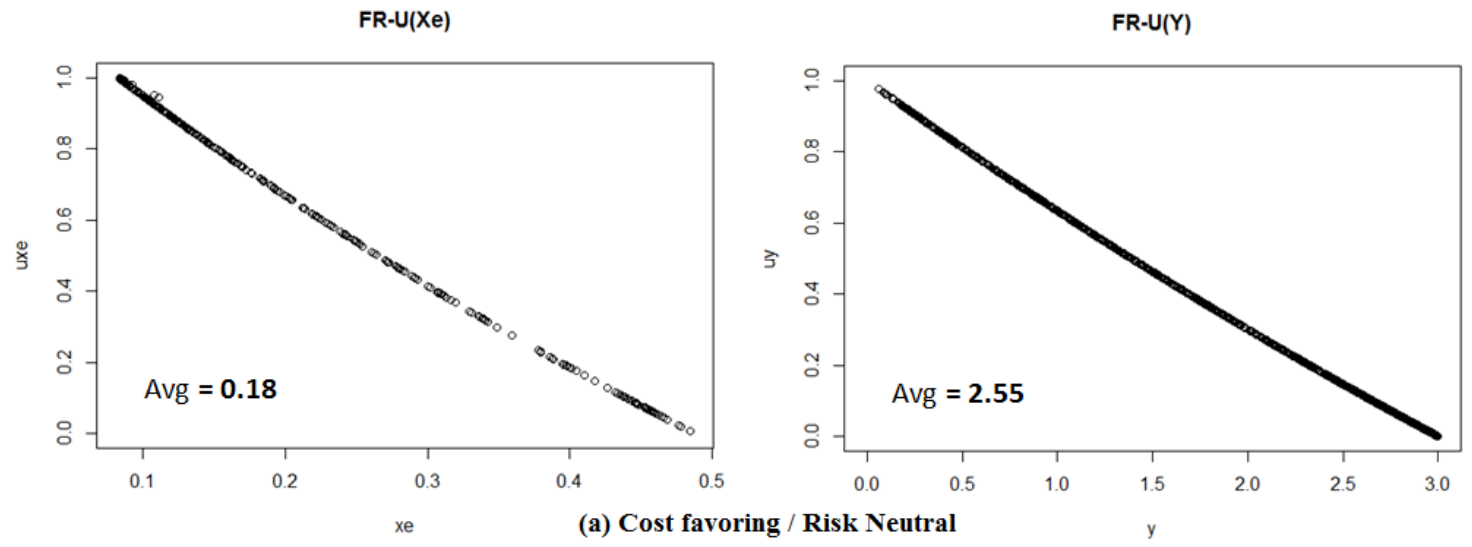

$F R \cdot U(X e)$
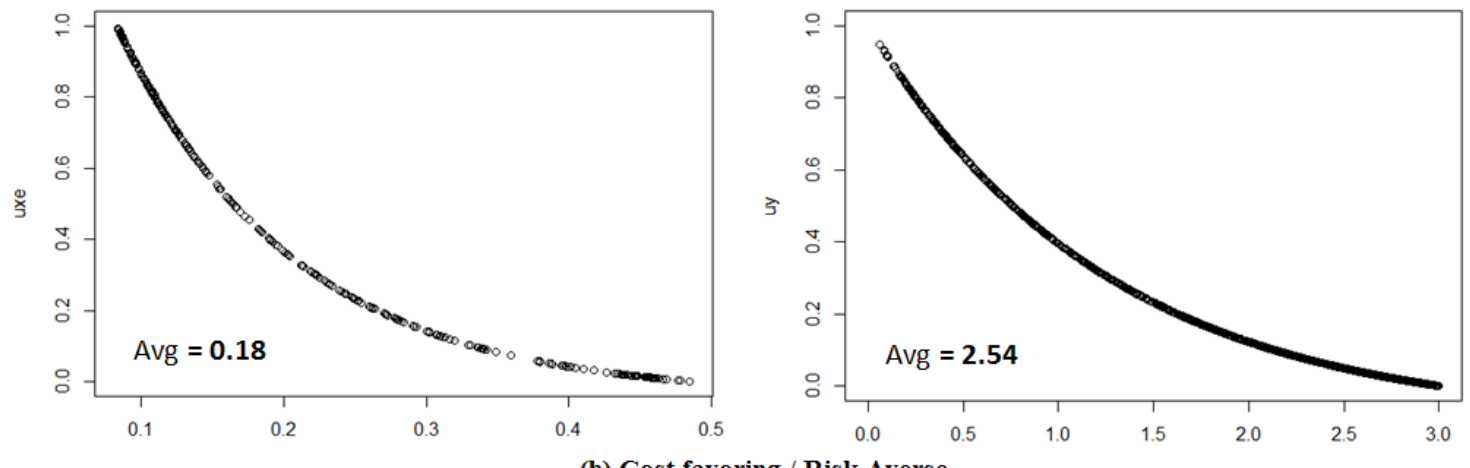

(b) Cost favoring / Risk Averse
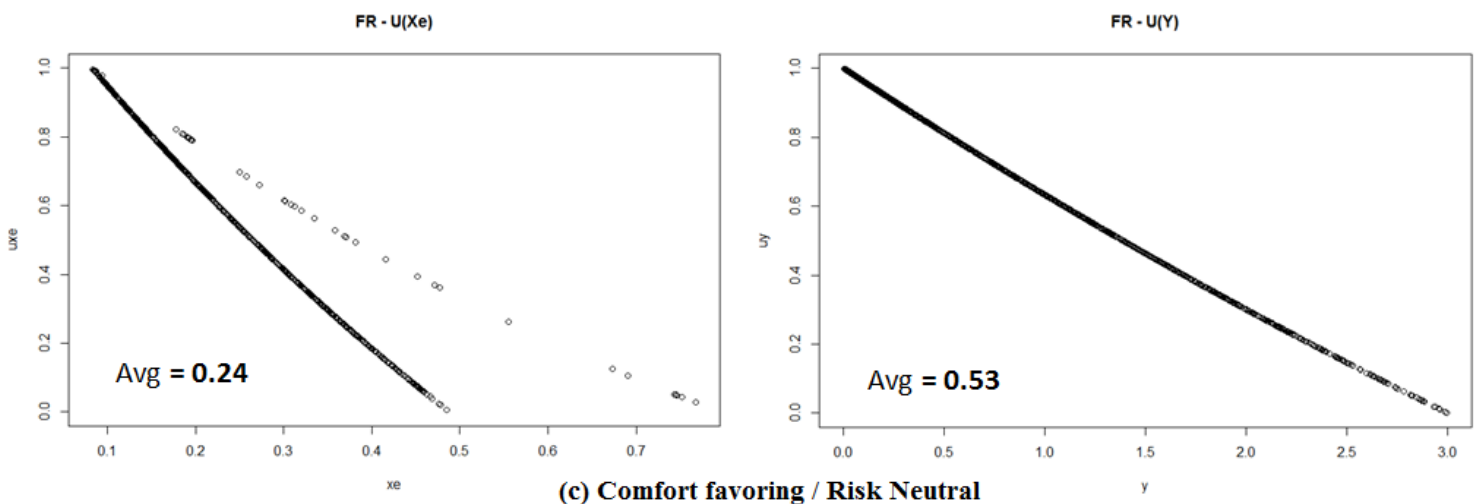

$F R \cdot U(X e)$
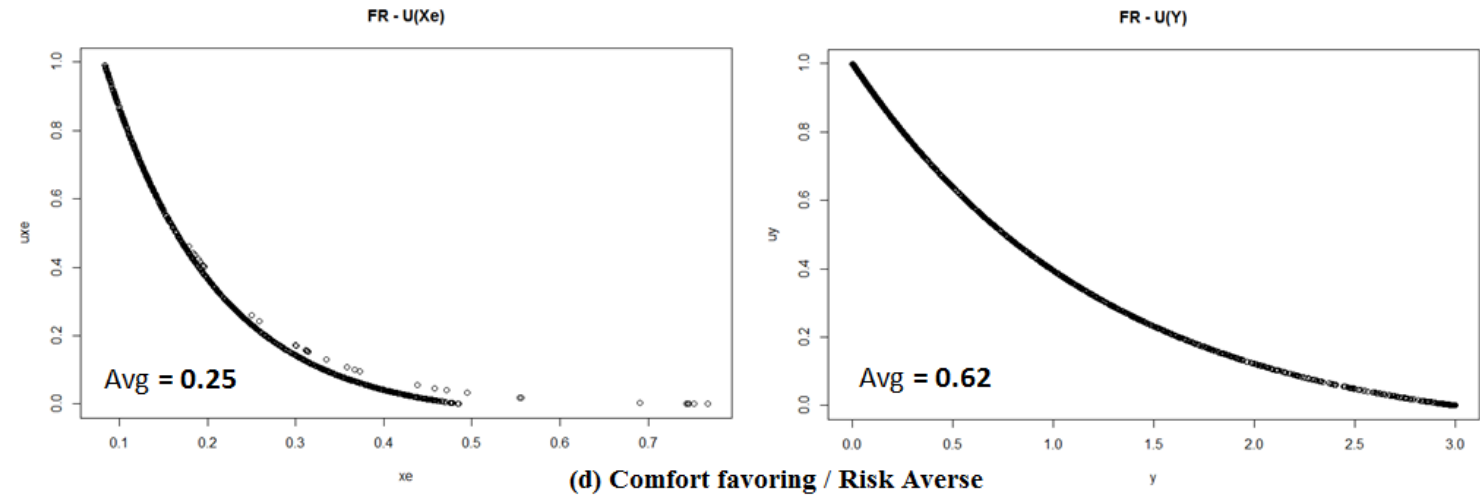
$\underline{\text { Time Of Use (TOU) }}$

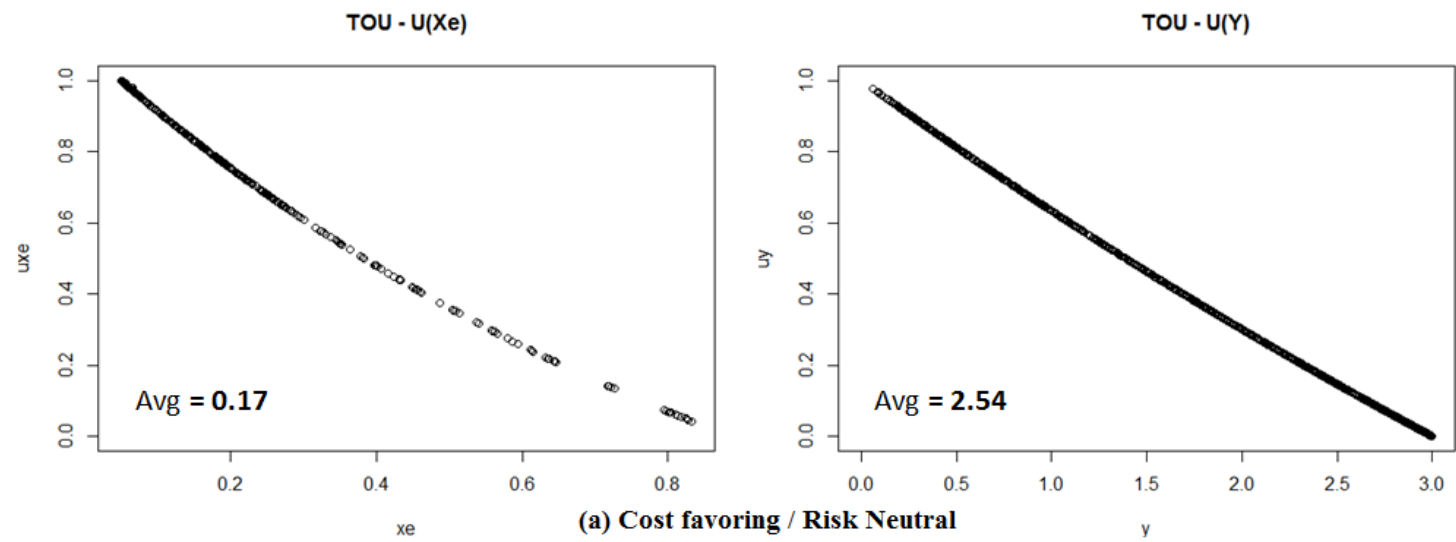

TOU - U(Xe)

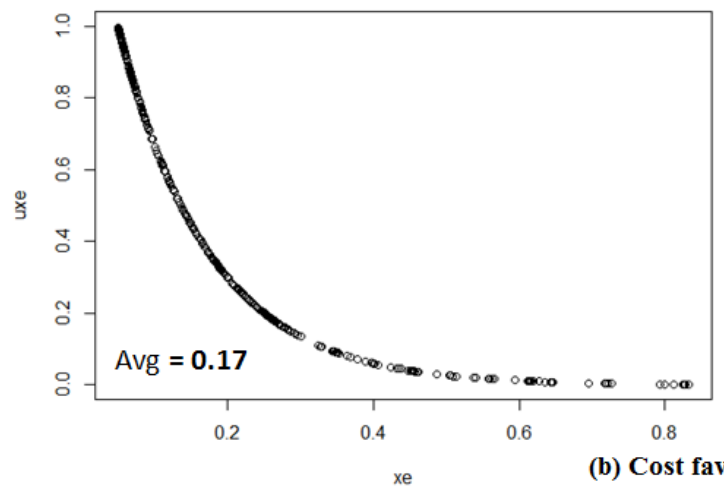

TOU - U(Y)

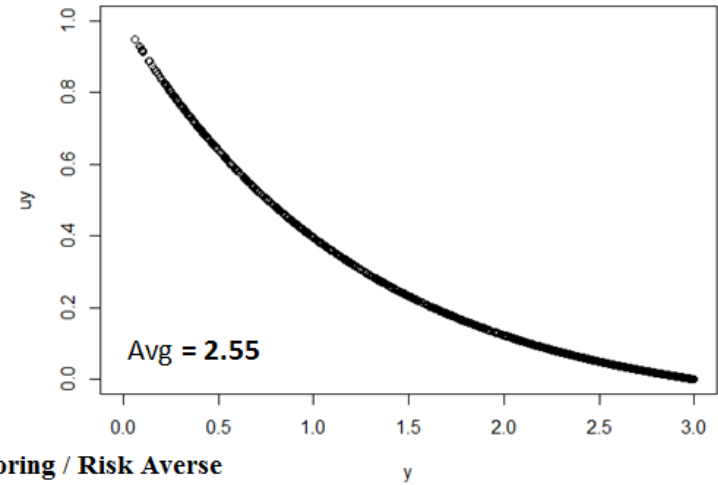

TOU - U(Xe)
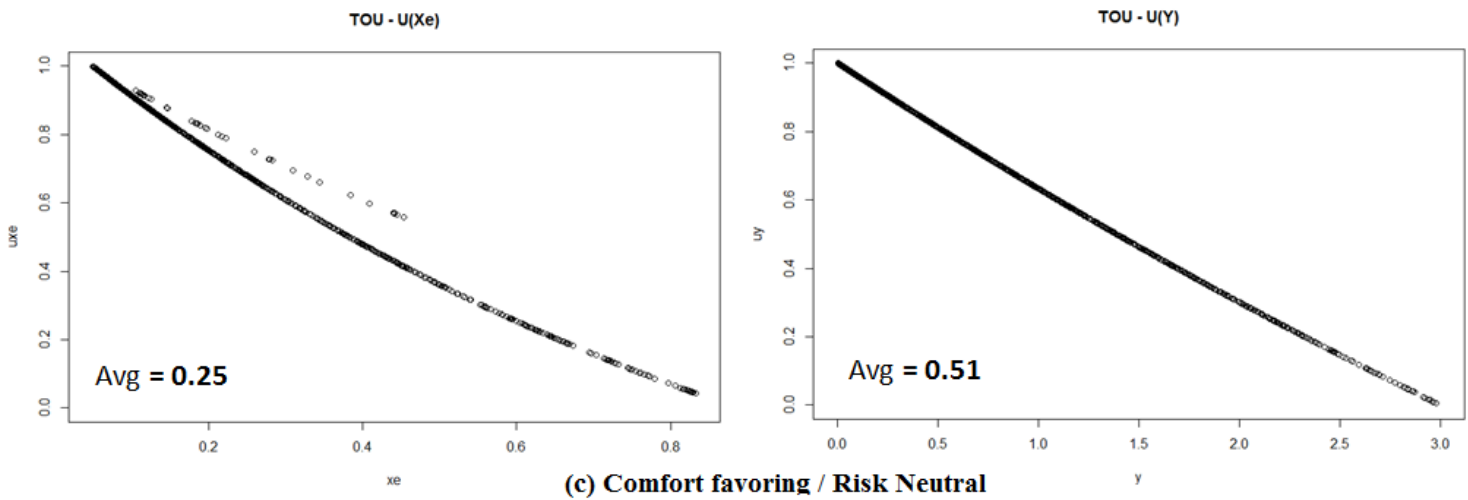

TOU - U(Xe)
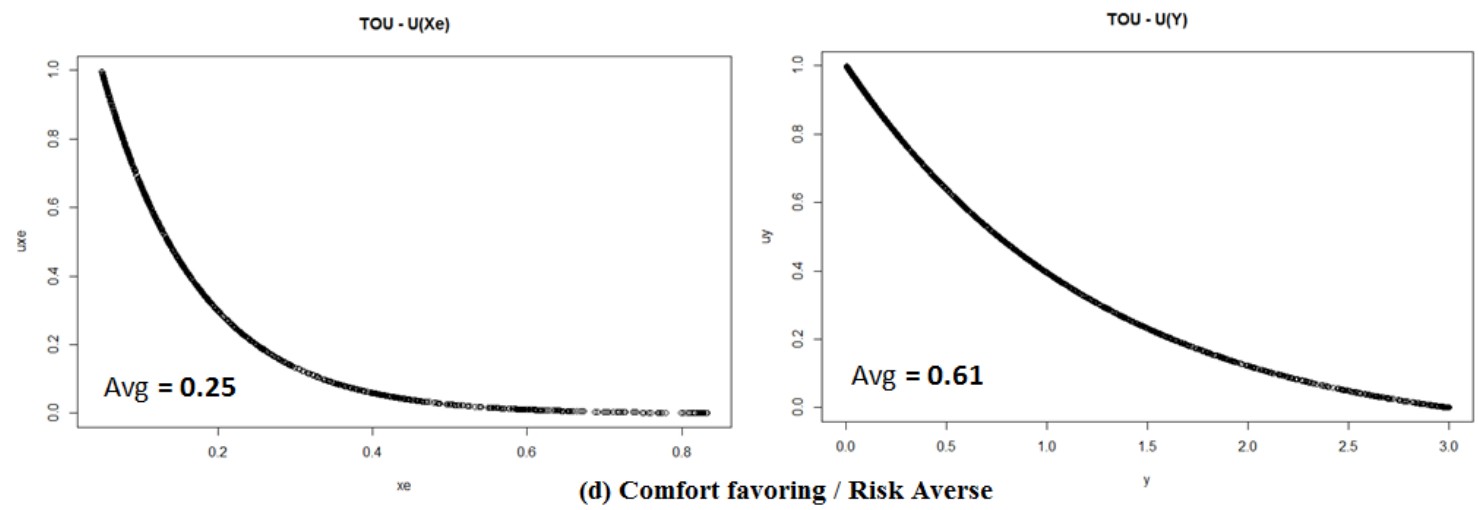


\section{Demand Charge on Coincident System Peak}
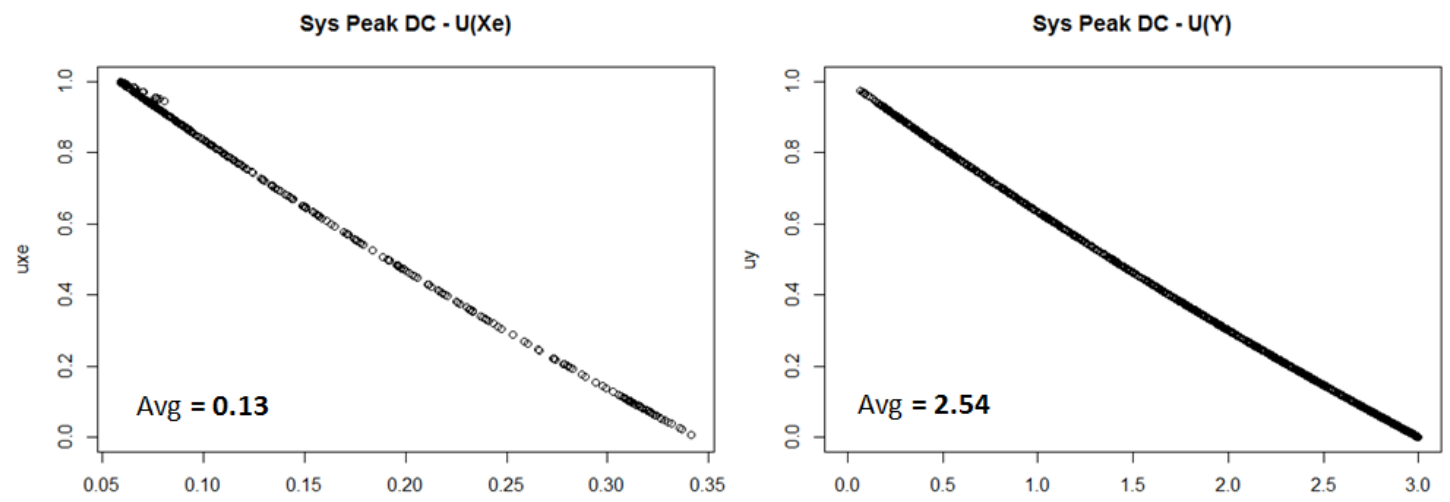

Sys Peak DC - U(Xe)

(a) Cost favoring / Risk Neutral
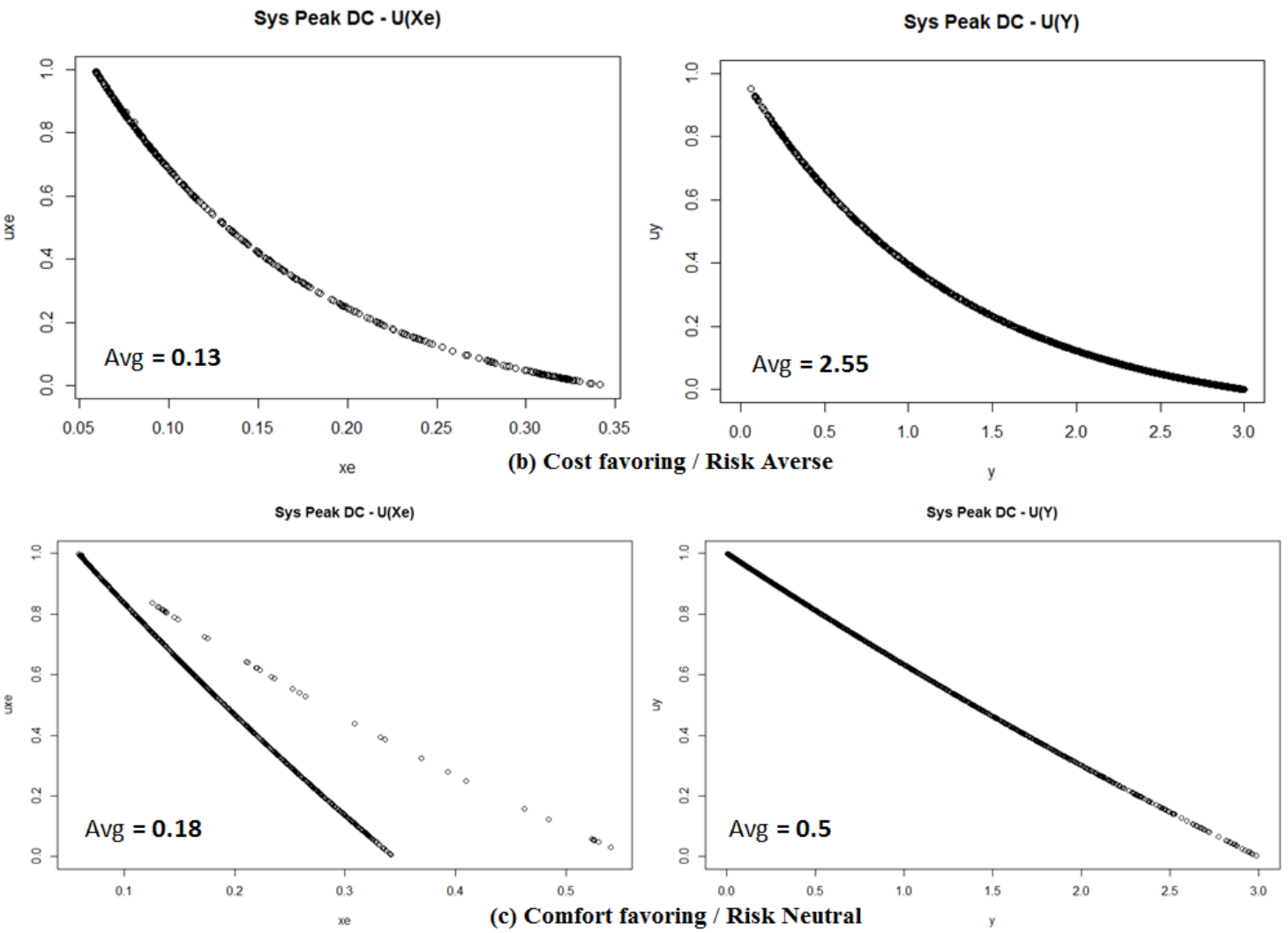

(c) Comfort favoring / Risk Neutral
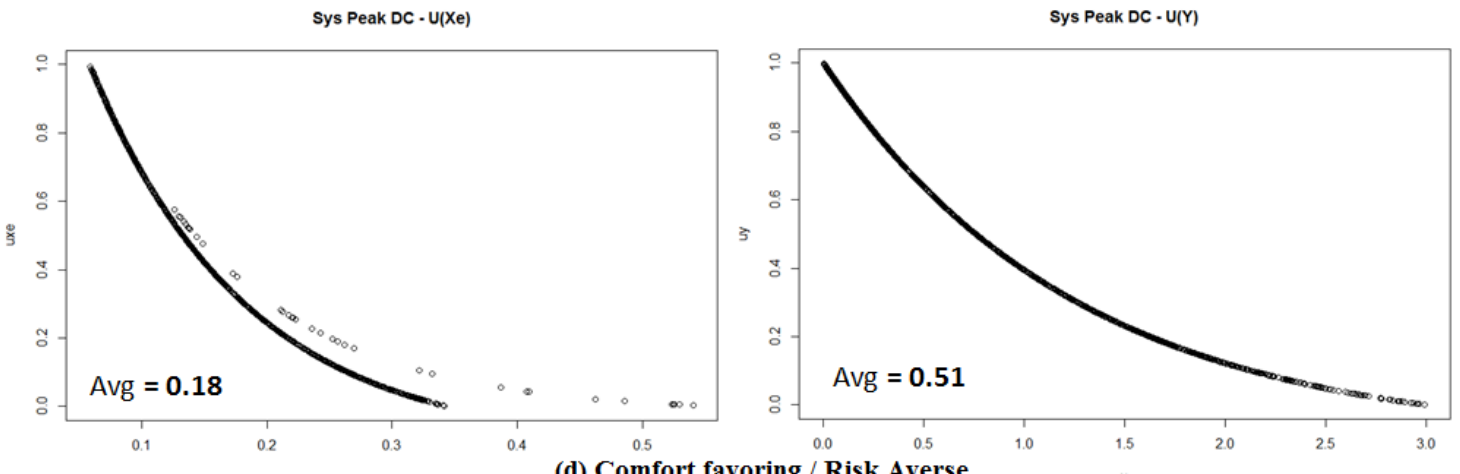

(d) Comfort favoring / Risk Averse 
APPENDIX B

Validating simulated system load against real system load for GEPB
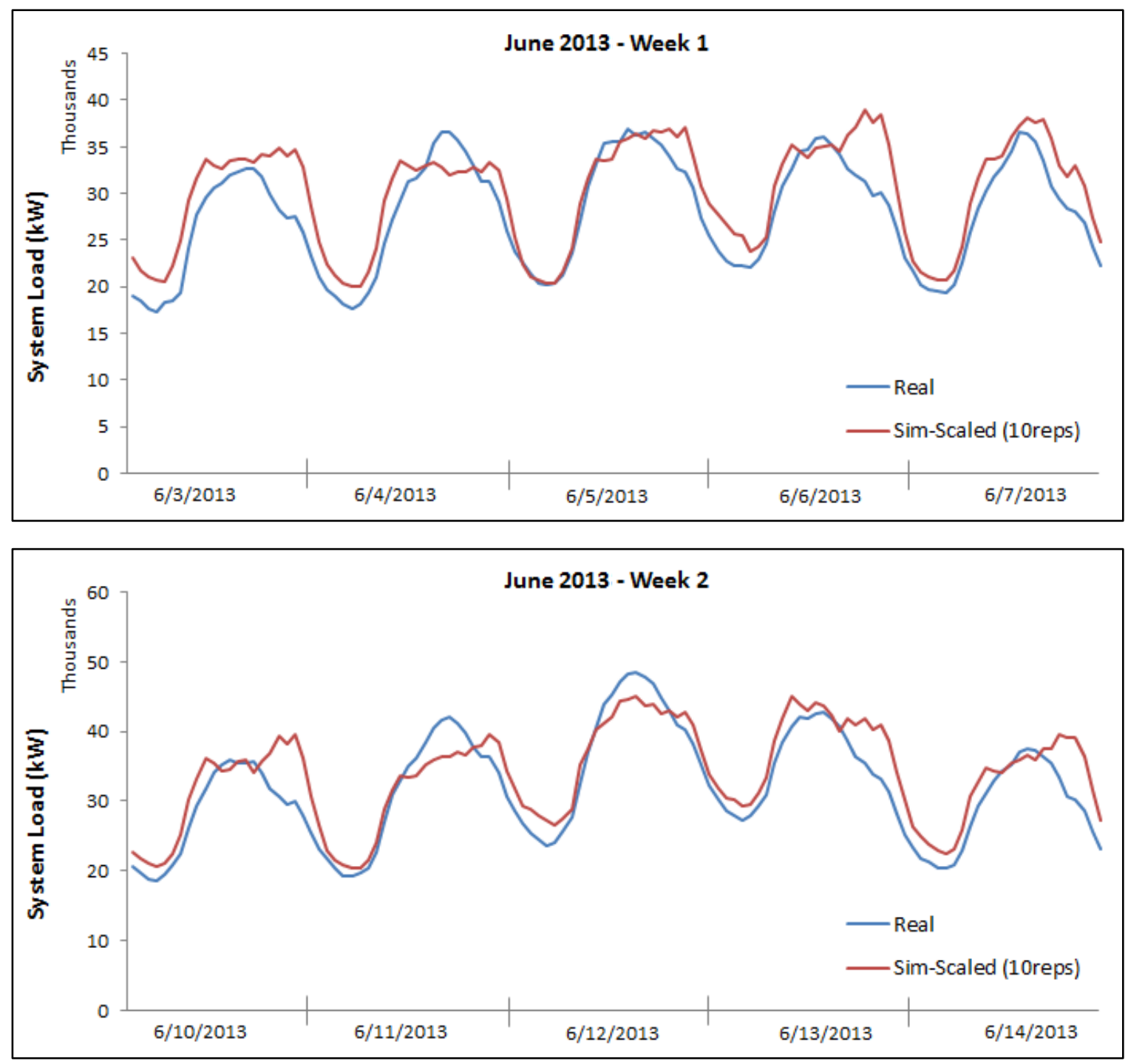

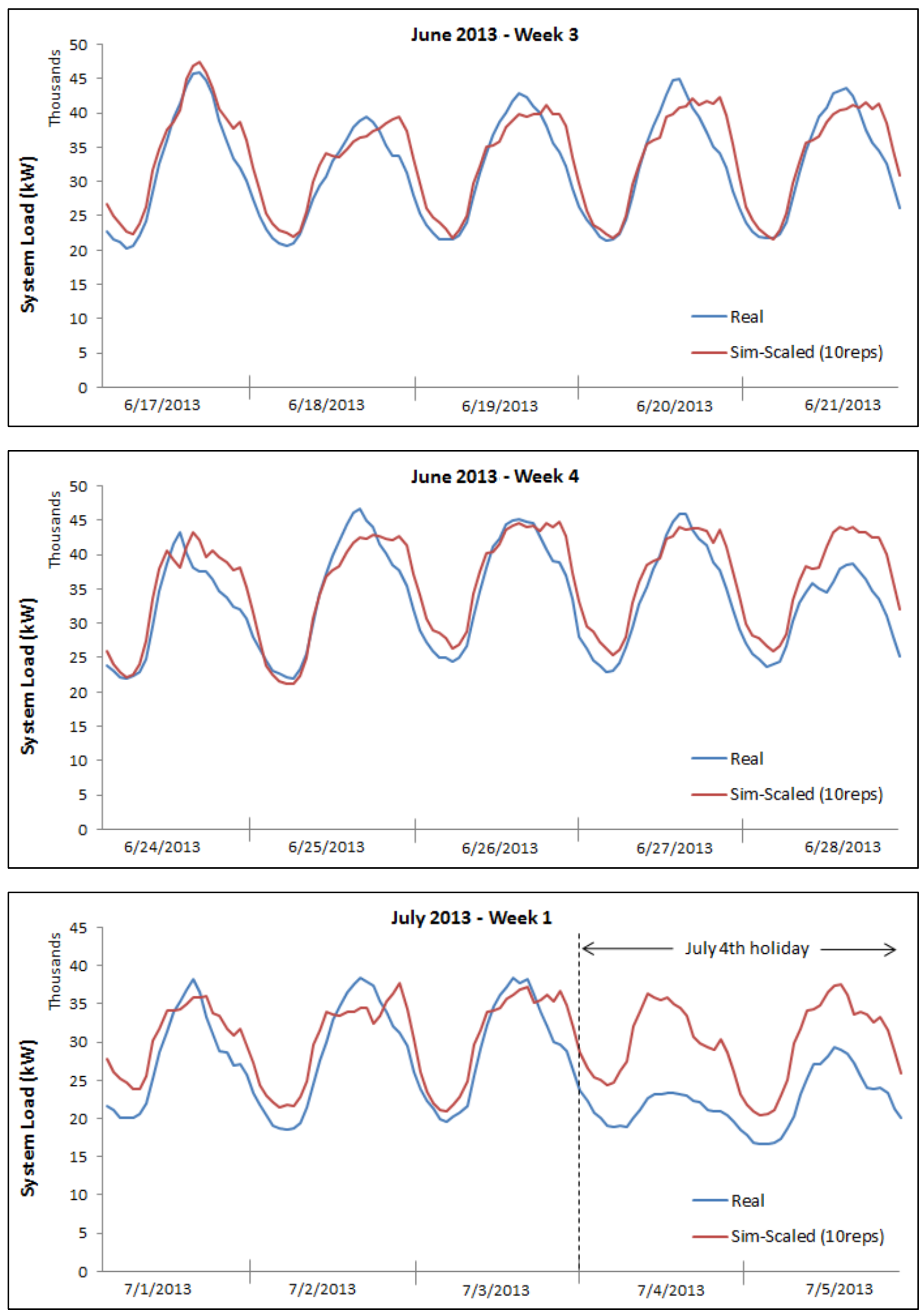

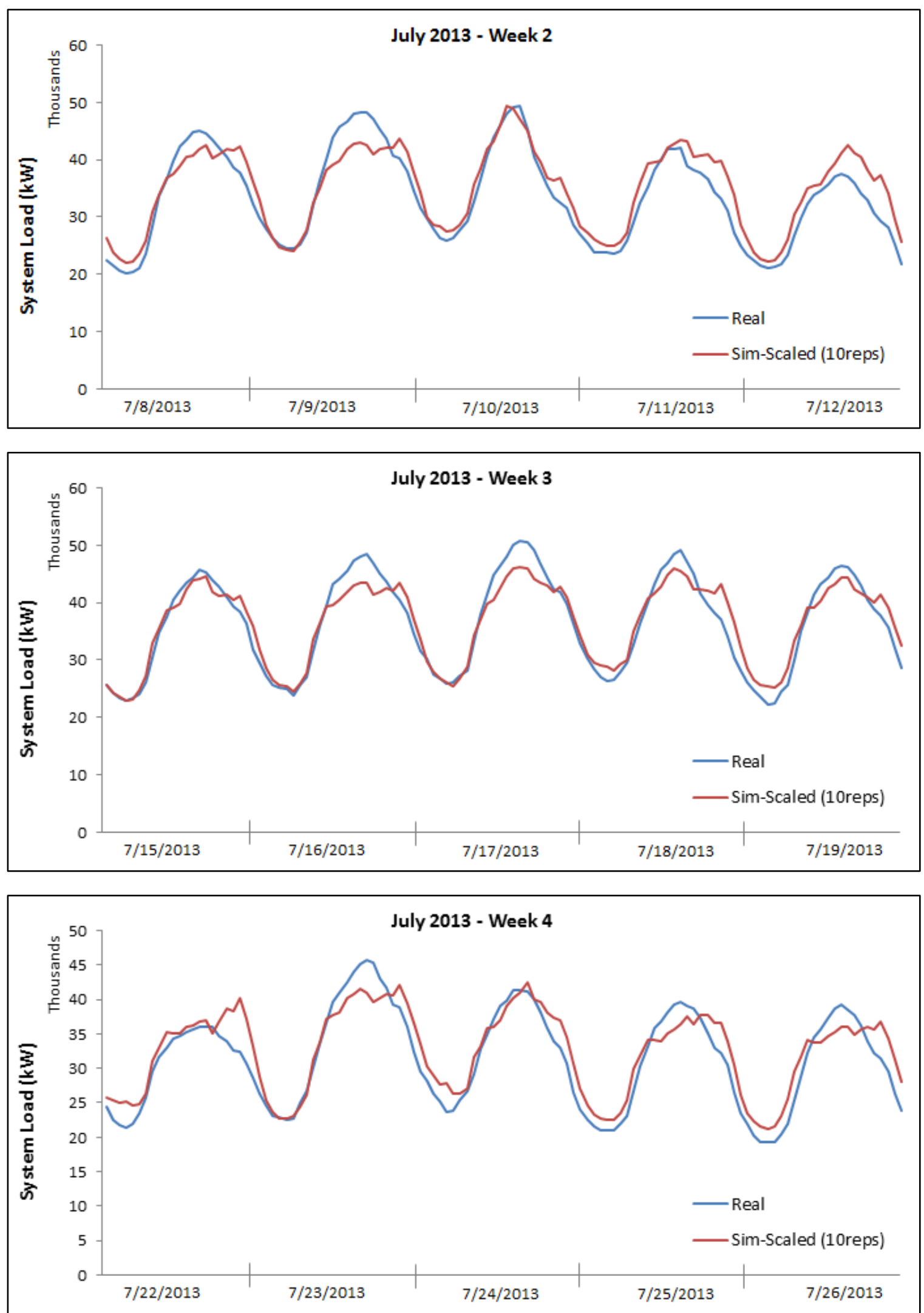

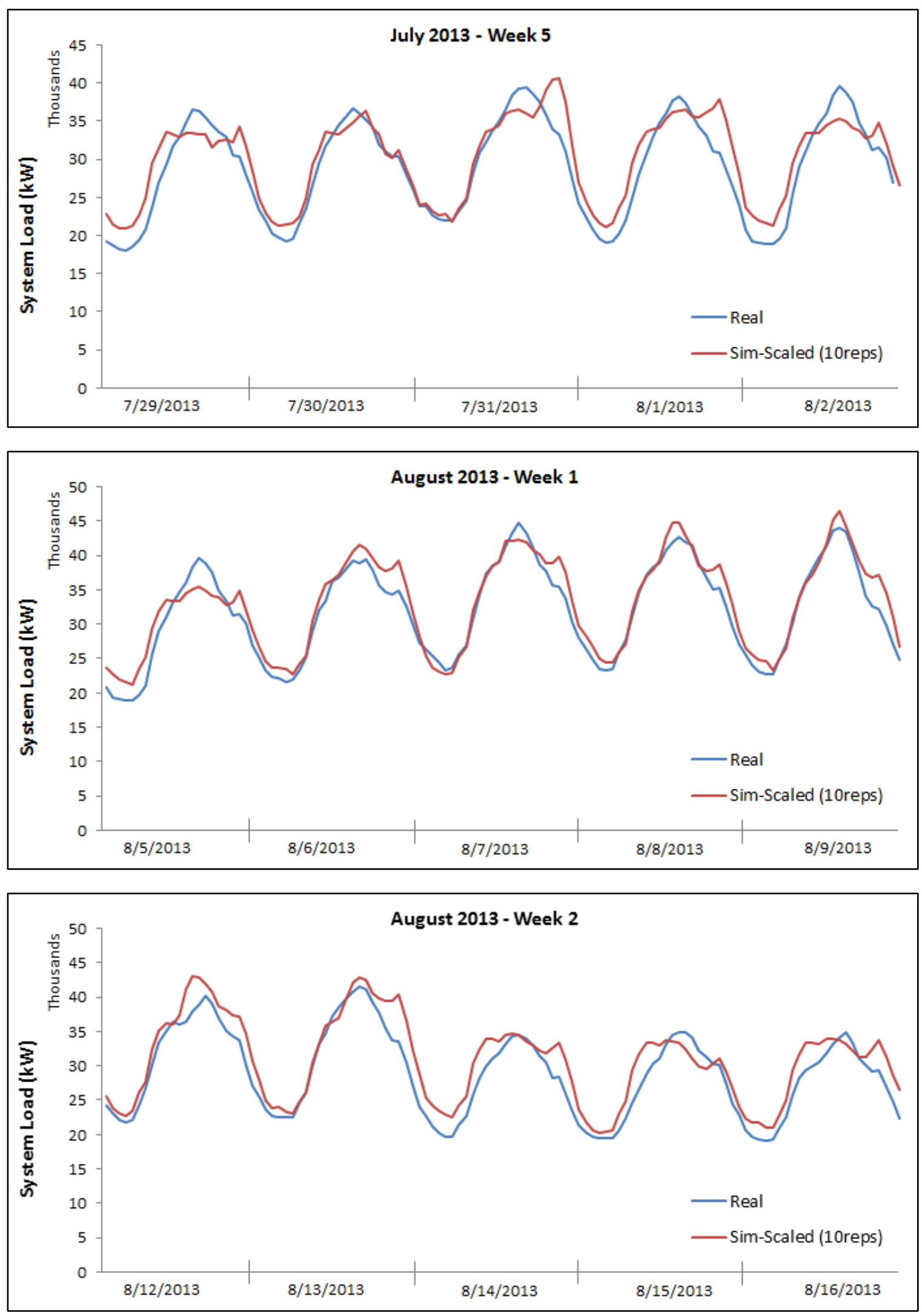

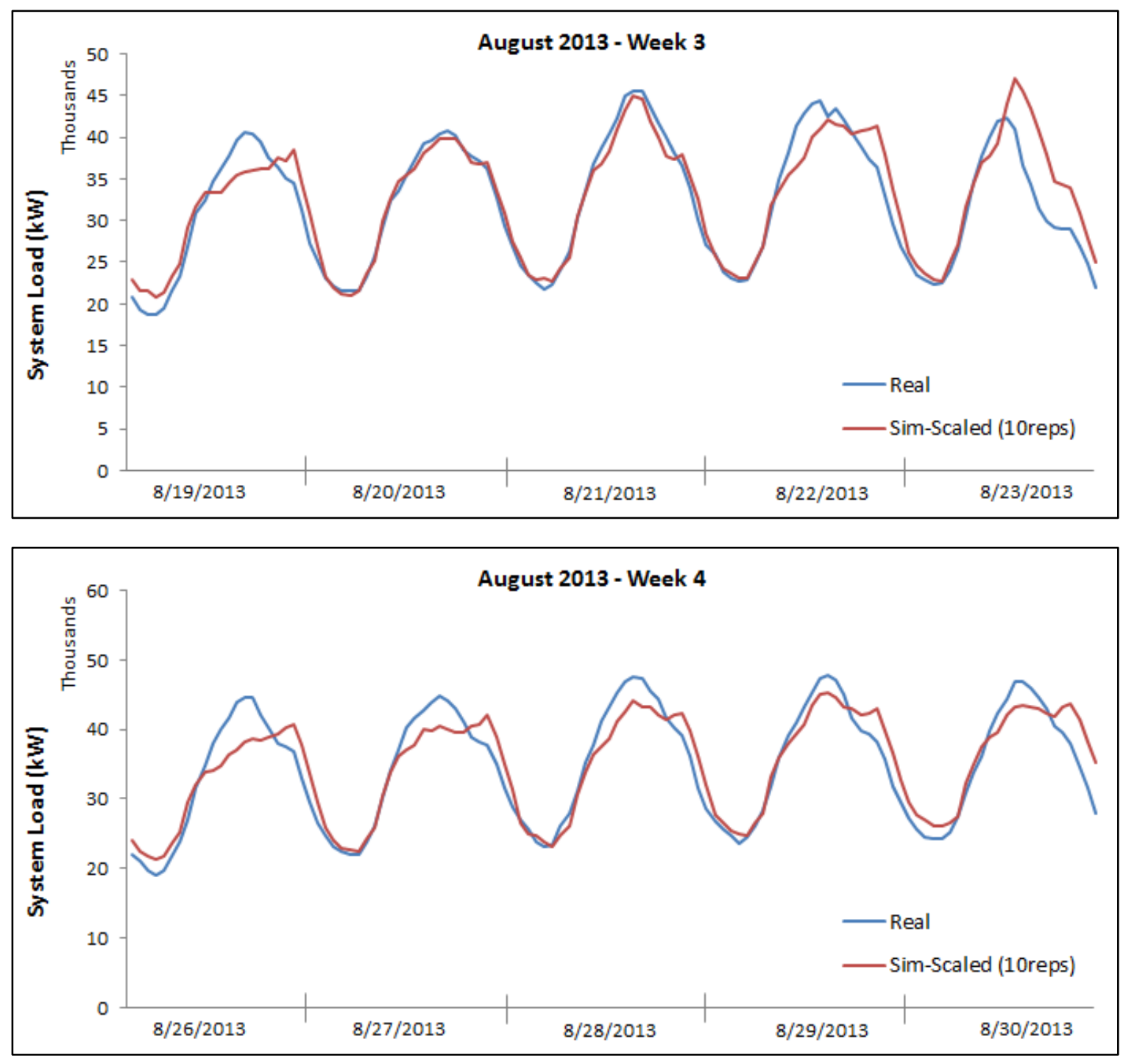


\section{CURRICULUM VITAE}

NAME: $\quad$ Prajwal Khadgi

ADDRESS: Department of Industrial Engineering Speed School of Engineering

University of Louisville

132 Eastern Pkwy

Louisville, KY 40292

DOB: $\quad$ Kathmandu, Nepal - May 28, 1983

EDUCATION

\& TRAINING: $\quad$ B.E., Mechanical Engineering

Tribhuvan University

Lalitpur, Nepal

$2001-2005$

M.S., Industrial Engineering

Northern Illinois University

DeKalb, IL

$2007-2009$

Ph.D., Industrial Engineering

University of Louisville

Louisville, KY

$2011-2016$

PROFESSIONAL SOCIETIES: Alpha Pi Mu

INFORMS

IIE

PUBLICATIONS: "Mapping Automated Pavement Data to Windshield Visual Survey Data: A Statistical Approach," 96 $6^{\text {th }}$ Annual Meeting of

Transportation Research Board. (2016)

"A Simulation Model with Multi-Attribute Utility Functions for Energy Consumption Scheduling in a Smart Grid," Energy

Systems, Volume 6, Issue 4, pp 533-550. (2015) 
"Using Agent Based Simulation and Model Predictive Control to Study Energy Consumption Behavior Under Dynamic Pricing," Proceedings of the 2014 Winter Simulation Conference. (2014)

"Modeling Demand Response Using Utility Theory and Model Predictive Control," Proceedings of the 2014 Industrial and Systems Engineering Research Conference. (2014)

"Simulation in Retail: A Case Study for Process Improvement in the Receiving Area," Proceedings of the 2009 Winter Simulation Conference. (2009)

"Simulation Analysis of Passenger Check In and Baggage Screening Area at Chicago Rockford International Airport," NIU Engineering Review, Vol 1, Issue 1, Pg 29. (2009) 